\title{
A Level Set Approach to Integer Nonlinear Optimization
}

Dissertation

zur Erlangung des mathematisch-naturwissenschaftlichen Doktorgrades

"Doctor rerum naturalium"

der Georg-August-Universität Göttingen

im Promotionsprogramm Mathematik (SMS)

der Georg-August University School of Science (GAUSS)

vorgelegt von

Ruth Hübner

aus München

Göttingen, 2013 


\section{$\underline{\text { Betreuungsausschuss }}$}

Prof. Dr. Anita Schöbel, Institut für Numerische und Angewandte Mathematik, GeorgAugust-Universität Göttingen

Jun.-Prof. Dr. Stephan Westphal, Institut für Numerische und Angewandte Mathematik, Georg-August-Universität Göttingen

\section{$\underline{\text { Mitglieder der Prüfungskommission }}$}

Referentin: Prof. Dr. Anita Schöbel, Insitut für Numerische und Angewandte Mathematik, Georg-August-Universität Göttingen

Koreferent: Prof. Dr. Christoph Buchheim, Fakultät für Mathematik, Technische Universität Dortmund

Weitere Mitglieder der Prüfungskommission:

Prof. Dr. Stefan Halverscheid, Mathematisches Institut, Georg-August-Universität Göttingen

Prof. Dr. Gerlind Plonka-Hoch, Institut für Numerische und Angewandte Mathematik, GeorgAugust-Universität Göttingen

Prof. Dr. Dominic Schuhmacher, Institut für Mathematische Stochastik, Georg-AugustUniversität Göttingen

Jun.-Prof. Dr. Stephan Westphal, Institut für Numerische und Angewandte Mathematik, Georg-August-Universität Göttingen

Tag der mündlichen Prüfung: 22.10.2013 


\section{Preface}

Even though I spent a lot of time on my own in my office (especially in the last weeks), a thesis cannot be written without the help and support of a lot of people. Therefore I would like to take the opportunity to thank them.

First of all I feel the need to thank my supervisor Anita Schöbel for inspiring the interest in this topic, and in optimization in general, in me. After supervising my diploma thesis you gave me the chance to keep on working in this field and to investigate things in more detail and not least you gave me the idea to do a $\mathrm{PhD}$ in the first place. I would like to thank you for a lot of joint discussions and work, for giving me all the support I needed and for cheering me up when I lost my faith! I know that your door is always open.

Futhermore, I want to thank Christoph Buchheim and Anita for our joint work which is also reported in Chapter 5 of this thesis and Christoph for agreeing to be the second referee of my thesis without any hesitation.

Also I would like to thank Stephan Westphal for being my co-supervisor for the last three years and giving me good advice.

That I had such a great time the last three years it not only thanks to Anita but also due to my wonderful colleagues. I have to thank you all for the time we had together (not only at work), for a lot of support and new ideas and for your friendship. First of all I have to mention Marie Schmidt (Thanks for always being there!), Thorsten Krempasky (Thanks for always pointing out that there are more important things to worry about!) and Jonas Ide (Thanks for being a good officemate!) who were here for the whole three years that I was. One after the other Robert Schieweck (Thanks for proofreading all of my thesis and your latex support!), Marco Bender (Thanks for making me run the Altstadtlauf!), Morten Tiedemann (Thanks for coming early and providing milk!), Jonas Harbering (Thanks for your computer support!) and Sönke Behrends (Thanks for interesting discussions on your master thesis!) joined in and enriched our group. Furthermore, I had the chance to spent a lot of time with Marc Goerigk (Thanks for your help with the robust stuff!), who has left Göttingen by now and Denise Krempasky (Thanks for the good time we had!), who is inofficially also part of this group. Also I would like to thank the former members of our group Mark-Christoph Körner and Daniel Scholz for helping me getting started.

Next I really want to thank my parents Monika and Wolfgang Hübner for giving me all the support I could ever ask for, for always believing in me and for being interested in my weird 
math stuff. And my sisters Anna and Jule Hübner for always being there when I need you and Anna for proofreading almost 200 pages of cryptic stuff and 24/7 online English support. I thank all four of you for understanding that I had mainly my thesis in my mind in the last weeks and for cheering me up whenever it was necessary!

Furthermore, I feel the need to thank my friends (especially Kathrin Deppe, Arne Grimsel, Birgit and Marco Sebexen) for your understanding, for listening to my worries and for bringing other things to my mind.

Last but not at all least I really have to thank Robert for always being there for me, for listening (again and again) to all my worries and for your understanding! But also for the best proofreading one could ever wish for, a lot of input to this thesis, many discussions and of course your perfect latex and asymptote support!

\section{Ich bin Euch allen so dankbar!}




\section{Contents}

$\begin{array}{ll}\text { Preface } & \text { i }\end{array}$

1. Introduction 1

1.1. Mixed-integer nonlinear optimization . . . . . . . . . . . . . . 3

1.2. Basic concepts and notations . . . . . . . . . . . . . . . 8

1.2.1. The Rounding Property . . . . . . . . . . . . . . . . . 8

1.2.2. Level set approach . . . . . . . . . . . . . . . . . . . . . . . . . . . . . . . . . . . 14

1.3. Round sets . . . . . . . . . . . . . . . . . . . 17

I. Sufficient conditions for the Rounding Property 21

2. Quasi-round sets 23

2.1. Properties of quasi-round sets . . . . . . . . . . . . . . . 23

2.2. Quasi-round sets and the Rounding Property . . . . . . . . . . . . 26

2.3. Applications . . . . . . . . . . . . . . . . . . . . 40

2.3.1. Perturbed p norms . . . . . . . . . . . . . . . . . 40

2.3.2. Strongly convex functions with Lipschitz-continuous gradient . . . . . . 42

2.3.3. Robust optimization . . . . . . . . . . . . . . . . . . . . . 42

2.3.4. Quasi-round ellipsoids . . . . . . . . . . . . . . . . 46

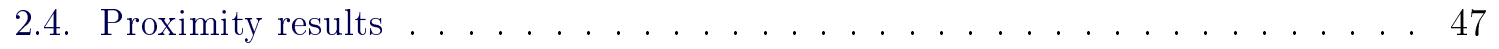

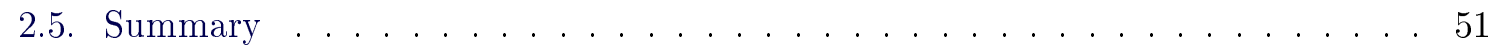

3. Cross-shaped sets 53

3.1. Properties of cross-shaped sets . . . . . . . . . . . . . . 53

3.2. Cross-shaped sets and the Rounding Property . . . . . . . . . . . . . . 58

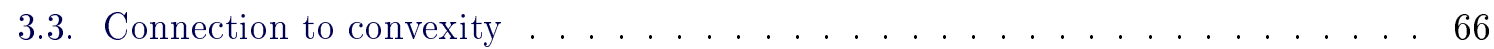

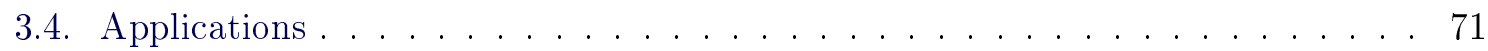

3.4.1. Separable quasiconvex functions . . . . . . . . . . . . . . 71

3.4.2. Norms . . . . . . . . . . . . . . . . . . . . . . . 75

3.4.3. Location theory . . . . . . . . . . . . . . . . . . 79 
3.5. Quasi-cross-shaped sets . . . . . . . . . . . . . . . 85

3.6. Summary . . . . . . . . . . . . . . . . . . . 88

4. Comparison and generalizations $\quad 89$

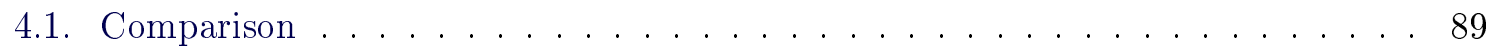

4.2. Generalization . . . . . . . . . . . . . . . . . . 91

$\begin{array}{ll}\text { II. Applications and extensions } & 97\end{array}$

5. Lower bounds and algorithms for convex quadratic integer programs 99

5.1. Convex quadratic integer programs . . . . . . . . . . . . . . . . . 99

5.2. Axisparallel, quasi-round and quasi-axisparallel ellipsoids . . . . . . . . . . . . 103

5.3. Lower bounds for (CQIP) . . . . . . . . . . . . . . . . . . . 112

5.4. Lower bounds to use in a branch-and-bound approach . . . . . . . . . . . . . 120

5.4.1. Worst-case analysis . . . . . . . . . . . . . . . . . . 121

5.4.2. Average-case analysis . . . . . . . . . . . . . . . . . . 122

5.5. Comparison of the bounds . . . . . . . . . . . . . . . . . 124

5.6. Summary . . . . . . . . . . . . . . . . . . . . . . . . 132

6. Extension to mixed-integer problems 133

6.1. The mixed-integer case . . . . . . . . . . . . . . . . . . . 133

6.2. Connection to the pure integer case . . . . . . . . . . . . . . 138

6.3. A mixed-integer relaxation to pure integer problems . . . . . . . . . . . . 145

6.4. Application to convex quadratic problems . . . . . . . . . . . . . . . 148

6.5. Summary . . . . . . . . . . . . . . . . . . . . . 153

7. Conclusion 155

7.1. Summary . . . . . . . . . . . . . . . . . . . 155

7.2. Further research directions . . . . . . . . . . . . . . . . . . . . . 158

$\begin{array}{lr}\text { Frequently used notation } & 161\end{array}$

$\begin{array}{ll}\text { Summary of contributions } & 163\end{array}$

$\begin{array}{ll}\text { References } & 163\end{array}$

$\begin{array}{ll}\text { Index } & 171\end{array}$ 


\section{Introduction}

Integer and mixed-integer nonlinear optimization problems occur in many applications, may they be real world or academic, compare e.g. Nemhauser and Wolsey [1988], Grossmann and Kravanja [1995] or Jünger et al. [2010]. Unfortunately, they combine two aspects that add, each one separately, an additional challenge to the problem: namely the nonlinearity of the objective function and the integrality constraint. This means also that integer nonlinear optimization problems are NP-hard as they contain the class of integer optimization problems and the class of nonlinear optimization problems, which are both NP-hard. While one can think of special cases where the combination of these two might lead to a simpler problem, for example if there are only a constant number of feasible integer points, in general it is to be expected that integer nonlinear optimization problems are even harder to solve than nonlinear or integer optimization problems. (See also Section 1.1 for more details.)

A basic assumption that we use throughout this work is that it is easier to solve the continuous relaxation (this is if we skip the integrality constraint) than the original integer problem. Although there might be exceptions this is a pretty common assumption. If we assume that it is natural to ask "Why not just solve the continuous relaxation and round the obtained solution?". This would make live so much easier! Of course this is not going to lead to an optimal solution to the integer problem in general - often not even to a feasible one. But if it leads to an optimal solution it would be great to know it, because then it could speed up finding an optimal integer solution via an optimal solution to the continuous relaxation.

The goal of this thesis is to identify special cases where rounding an optimal solution to the continuous relaxation leads to an optimal solution of the integer problem - we call this the "Rounding Property" (see Section 1.2).

The basic idea followed in this work is described in the following example: assume that we know the (unique) optimal solution to the continuous relaxation (and that it is not integer itself) and that we also know that the level sets are Euclidean balls. Then we know that an optimal solution to the integer problem is the first integer point (or the first integer points) that we reach by increasing the level of the objective function (starting with the continuous optimal objective value), i.e., by growing the level sets. As the level sets are Euclidean balls this is going to be the closest integer to the optimal solution to the continuous relaxation. And hence this problem has the rounding property. Our task is to identify more general geometric shapes of level sets that also lead to this property. 
We need to make some rather strong assumptions on the problem to guarantee this kind of rounding property. But this is what is done most of the time to handle the very broad class of "integer nonlinear problems". Typical assumptions in the literature are for example convexity or concavity assumptions. (See also Section 1.1 for more details.) We do not need any of these but instead we impose others. In this way our results are alternative properties, in contrast to for example convexity, that lead to easier/ faster solution approaches.

Of course, the question is whether these theoretical assumptions will be met by a problem. Therefore a part of this work is to show some applications and adaptions to special types of optimization problems.

This work is organized as follows: we start by giving a glance on the literature on mixed-integer nonlinear optimization, followed by the introduction of basic concepts and notations that we use in the following and end this first chapter by explaining the already mentioned basic example in more detail and generality. This provides the basic starting point for everything that is to come.

Part I of this work is then dedicated to the task of finding other geometric shapes, like Euclidean balls, that guarantee the Rounding Property. We present basically two different ideas namely quasi-round (Chapter 2) and cross-shaped sets (Chapter 3). The corresponding chapters are organized parallel: after giving the formal definition and some basic properties, we establish the connection to the Rounding Property, show some applications and end with a generalization in each case. In Chapter 4 we give some general remarks and generalizations that are not limited to either case and show an alternative way of defining a rounding property. Part II is then on applications and extensions: in Chapter 5 we apply our theory to the special case of convex quadratic integer problems and show how to use the ideas presented so far to derive lower bounds even if the problem does not have the Rounding Property. These bounds are embedded in a branch-and-bound framework. Some first computational results indicate their possibilities and compare them among each other. Chapter 6 shows how the concept of the Rounding Property and our results can be adopted to the case of mixed-integer nonlinear optimization problems. This gives rise to an easy to calculate mixed-integer relaxation bound in many cases and is also applied to the convex quadratic case.

An overview on the dependencies between the chapters is given in the following picture: Chapter 1 is the basis for all that follows, Chapters 2 and 3 can be read without knowledge of the other one and Chapter 4 is based up on both of them. For Chapters 5 and 6 both 2 and 3 are needed and Chapter 5 might be helpful for Chapter 6 .

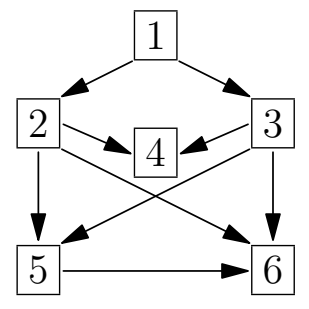

We end this work by giving a summary of the main ideas and results we develop and some further research ideas.

Parts of this work are joint work with coauthors, see the summary of contributions. 


\subsection{Mixed-integer nonlinear optimization}

The purpose of this section is to give a glance on complexity results and solution approaches for mixed-integer nonlinear optimization problems. The general mixed-integer nonlinear problem is of the following form:

$$
\begin{array}{lll}
(M I N L P) & \min & f\left(x_{1}, \ldots, x_{n}\right) \\
\text { s.t. } & g\left(x_{1}, \ldots, x_{n}\right) \leq 0 \\
& x \in \mathbb{Z}^{n_{1}} \times \mathbb{R}^{n_{2}}
\end{array}
$$

where $f: \mathbb{R}^{n_{1}+n_{2}} \rightarrow \mathbb{R}$ and $g: \mathbb{R}^{n_{1}+n_{2}} \rightarrow \mathbb{R}^{m}$.

Overviews of mixed-integer nonlinear optimization are for example provided by Grossmann [2002] and Hemmecke et al. [2010].

We start our overview by showing the complexity of the general mixed-integer nonlinear problem. To this end we observe first of all that continuous nonlinear programming is in general NP-hard: for example the problem of minimizing a quadratic function over box constraints, the so-called BoxQP-problem, is already NP-hard (see Pardalos and Vavasis [1991]). On the other hand also mixed-integer linear programming is NP-hard (see Garey and Johnson [1979]). Hence the class of mixed-integer nonlinear problems contains two NP-hard subclasses and is therefore also NP-hard in general. Furthermore, Jeroslow [1973] showed that the problem of minimizing a linear function over quadratic constraints over integer variables is incomputable. Therefore most approaches to solve these kind of problems are for special cases. We discuss the following two of these special cases here: mixed-integer linear optimization and mixedinteger convex optimization. But besides these there are of course also other mixed-integer optimization problems that have a special structure that allow for good solution strategies such as for example concave or binary problems. Furthermore, we only give exact approaches here since the scope of this work is on exact approaches, but it is of course noteworthy that the class of heuristics and approximation approaches for mixed-integer nonlinear problems is enormous. It is also to mention that there are a lot of approaches for special integer nonlinear optimization problems which we do not mention here.

Mixed-integer linear optimization We start by reviewing the mixed-integer linear case since this is the oldest and probably best-researched special case. Lenstra [1983] showed that in fixed dimension integer linear programming can be done in polynomial time. We present three ideas here to solve a mixed-integer linear problem because of their importance also for the mixed-integer nonlinear case. A detailed review on mixed-integer linear optimization and especially solvers for these problems is given by Lodi [2010].

The first idea was originally proposed by Land and Doig [1960] and is known today as branchand-bound. The basic idea is to start by solving the continuous relaxation of the mixed-integer problem, i.e., the problem we get if we relax the integrality constraints, which is a continuous linear problem. If the solution $\bar{x}$ we get to the continuous relaxation is already feasible for the mixed-integer problem we are done. Otherwise we construct two subproblems by choosing one variable $i \in\left\{1, \ldots, n_{1}\right\}$ s.t. $\bar{x}_{i} \notin \mathbb{Z}$ and adding to the original problems on the one hand the constraint $x_{i} \leq\left\lfloor\bar{x}_{i}\right\rfloor$ (where $\left\lfloor\bar{x}_{i}\right\rfloor$ is the greatest integer smaller than $\bar{x}_{i}$ ) and for the other problem the constraint $x_{i} \geq\left\lfloor\bar{x}_{i}\right\rfloor+1$. This means that $\bar{x}$ is not feasible for any of these two new problems. For each of the new problems we solve again the continuous relaxations and 
continue as described. The subproblems are organized in a tree structure to demonstrate their dependencies. Therefore the construction of the two subproblems is called branching. As soon as we get an integer solution to one of the subproblems it gives us an upper bound on the optimal objective value. This can be used to evaluate other subproblems: if the objective value of the continuous relaxation is already worse than the current upper bound we do not have to branch this problem any further since all subproblems of this problem will perform even worse and hence we discard this subproblem - this is the bounding (the same is true if a subproblem is infeasible). By proceeding in this way the subproblems become smaller and smaller as we restrict the feasible region and at some point the continuous relaxation produces integer values. As soon as all subproblems are either discarded or solved to optimality we are done.

Dakin [1965] presented a version of this basic algorithm designed to be easier to implement and furthermore no longer bound to integer linear problems but also applicable to integer nonlinear instances. An overview of advances in linear programming-based branch-and-bound approaches since these basic works is given for example by Johnson et al. [2000].

At the same time as Land and Doig, Gomory [1958] came up with a different idea to solve pure integer linear problems: the cutting plane algorithm. His idea is: if the continuous relaxation of an integer linear optimization problem is not integer itself we add a new constraint to this problem that is satisfied by all feasible integer points but not by the optimal solution to the continuous relaxation. This new inequality is called a cut since it cuts off the continuous optimal solution. The cut originally introduced by Gomory, the Gomory cut, is based on the following observation: using the simplex method to solve the continuous relaxation we are left with a set of equations of the form $x_{B(j)}=b_{j}^{\prime}-\sum_{i=1}^{n-m} a_{j, N(i)}^{\prime} x_{N(i)}$ for the basis variables $x_{B}$ and the non-basis variables $x_{N}$ and get the simplex solution $x_{B(j)}=b_{j}^{\prime}$ and $x_{N(i)}=0$. If one of the $b_{j}^{\prime}$ is not integer this solution is not a solution to the integer problem. We can reformulate this equation as

$$
x_{B(j)}+\sum_{i=1}^{n-m}\left\lfloor a_{j, N(i)}^{\prime}\right\rfloor x_{N(i)}-\left\lfloor b_{j}^{\prime}\right\rfloor=\underbrace{b_{j}^{\prime}-\left\lfloor b_{j}^{\prime}\right\rfloor}_{<1}-\underbrace{\sum_{i=1}^{n-m}\left(a_{j, N(i)}^{\prime}-\left\lfloor a_{j, N(i)}^{\prime}\right\rfloor\right) x_{N(i)}}_{\geq 0} .
$$

This means, for all $x \in\left(\mathbb{Z}_{0}^{+}\right)^{n}$ the left-hand side is integer and the right side is less than 1 . This means that

$$
b_{j}^{\prime}-\left\lfloor b_{j}^{\prime}\right\rfloor-\sum_{i=1}^{n}\left(a_{j, i}^{\prime}-\left\lfloor a_{j, i}^{\prime}\right\rfloor\right) x_{N(i)} \leq 0
$$

is feasible for all feasible $x \in \mathbb{Z}^{n}$ but not for our simplex solution $x_{B(j)}=b_{j}$ and $x_{N(i)}=0$ and hence a cut of the required form.

Adding cuts to the original problem means shrinking the feasible region of its continuous relaxation. Therefore the cutting plane algorithm geometrically means to align the feasible region more and more with the convex hull of the feasible integer points.

A strong approach is the combination of both approaches, the so-called branch-and-cutalgorithms. The idea is to add cuts either at the beginning or also while processing the tree to strengthen the continuous relaxations. Caprara and Fischetti [1997] give an overview of these approaches. 
The last approach for mixed-integer linear problems we mention here is the idea of test sets. Graver [1975] introduced this idea as a generalization of both the simplex method and the flow algorithm to general integer linear optimization. The idea is the following: An integral test set $T$ for a feasible $x \in F:=\left\{x \in \mathbb{Z}^{n}: A_{1} x=b_{1}, A_{2} x \leq b_{2}\right\}$ is a finite set of vectors, $T \subseteq\left\{t \in \mathbb{Z}^{n}: A_{1} t=0\right\}$, such that for all $t \in T \max \{\alpha: x+\alpha t \in F\}>0$ and for all $y \in F$ $y-x=\sum_{t \in T} \alpha_{t} t$ where $\alpha_{t} \in \mathbb{N}$. This gives rise to the following optimality criterion: if $T$ is an integral test set for $x \in F$ and if for all $t \in T$ we have $c^{t} t \geq 0$ then $x$ is an optimal solution to $\min \left\{c^{t} x: A_{1} x=b_{1}, A_{2} x \leq b_{2}, x \in \mathbb{Z}^{n}\right\}$ (compare Graver [1975]). This means, if we are given a test set $T$ and a feasible point $x$ we can design an augmentation algorithm that will terminate with an optimal solution if one exists. The original test set introduced by Graver [1975] is the so-called Graver basis

$$
\begin{aligned}
& \mathcal{G}(A):=\left\{t \in \mathbb{Z}^{n}: A_{1} t=0, \max \{\alpha: x+\alpha t \in F\}>0,\right. \\
& \text { for all } w \in \mathbb{Z}^{n}, A_{1} w=0 \text { s.t. }\left(a_{2}^{i} t\right)\left(a_{2}^{i} w\right) \geq 0 \text { and }\left|a_{2}^{i} t\right| \geq\left|a_{2}^{i} w\right| \text { for all rows } \\
& \left.a_{2}^{i} \text { of } A_{2} \text { follows } w=t \text { or } w=0\right\} \text {. }
\end{aligned}
$$

An overview of test sets for integer linear problems is given by Weismantel [1998].

Mixed-integer convex optimization For the special case of mixed-integer convex optimization the incomputable result does not hold since Khachiyan [1983], improved by Bank et al. [1991], showed that if $f$ and $g$ are quasi-convex polynomials of degree bound $d \geq 2$ there exists a radius $R \in \mathbb{N}$ such that

$$
\min \left\{f(x): g(x) \leq 0, x \in \mathbb{Z}^{n}\right\}=\min \left\{f(x): g(x) \leq 0,\|x\|_{2} \leq R, x \in \mathbb{Z}^{n}\right\} .
$$

Hence a global (however in general inefficient) approach to solve integer convex problems is to compare the objective values of all points in $\left\{x \in \mathbb{Z}^{n}: g(x) \leq 0,\|x\|_{2} \leq R\right\}$, which are only finitely many. In fixed dimension the problem of minimizing a convex function over mixed-integer variables in a convex set can be done in polynomial time as has been shown by Khachiyan [1983]. Nevertheless, mixed-integer convex problems are still NP-hard as they still contain the class of mixed-integer linear problems. The convexity of the objective function and the feasible region give rise to a large group of exact solution approaches of which we present a selection here. A recent survey on algorithms and software for mixed-integer convex problems is given by Bonami et al. [2012].

The branch-and-bound idea for the mixed-integer linear case can be applied also to the nonlinear case, as already mentioned by Dakin [1965]. The problem is then of course to solve the continuous relaxation, which was simply a linear problem in the former case, but is now a continuous nonlinear problem. The branch-and-bound approach was first applied to mixedinteger problems by Gupta and Ravindran [1985], who compare several branch-and-bound algorithms varying in branching rules and upper bound computation. An improvement of the original idea for mixed-integer convex problems is given for example by Borchers and Mitchell [1994] and Leyffer [2001]: the idea is not to solve the continuous relaxation to optimality for each subproblem, since this might be to expensive in the convex setting.

A cutting plane algorithm for mixed-integer convex problems has been introduced by Westerlund and Pettersson [1995]: the so-called extended cutting plane (ECP) algorithm, extending the method by Kelley [1960] to mixed-integer problems. A generalization to pseudo-convex 
problems is given by Westerlund and Pörn [2002]. Also branch-and-cut approaches have been extended to the convex setting, e.g. by Quesada and Grossmann [1992] or Stubbs and Mehrotra [1999].

Graver's idea of test sets has been generalized to some mixed-integer convex problems: Hemmecke [2003] derived a finite test set for the problem $\min \left\{f(x): A x=b, x \in \mathbb{Z}_{+}^{n}\right\}$ where $f(x):=\sum_{i=1}^{s} f_{i}\left(c_{i}^{t} x+c_{i, 0}\right)+c^{t} x$ for $f_{i}: \mathbb{R} \rightarrow \mathbb{R} \mathbb{Z}$-convex with minimum at 0 . A function $g: \mathbb{R} \rightarrow \mathbb{R}$ is called $\mathbb{Z}$-convex with minimum at 0 if $g(x+1)-g(x)$ in increasing in $x \in \mathbb{Z}$ and if $g(x+1)-g(x) \leq 0$ for all $x \leq 0$ and $g(x+1)-g(x) \geq 0$ for all $x \geq 0$. This means that for example the problem of minimizing a separable convex function over linear equation is contained in this class of problems. This result is extended to a broader class by Lee et al. [2008].

Besides these adaptions from the linear case, there are also some approaches specifically designed for mixed-integer convex optimization. For improved readability we rewrite (MINLP) in the following way

$$
\begin{array}{lcl}
(M I N L P) & \min & f(x, y) \\
\text { s.t. } & g(x, y) \leq 0 \\
& x \in X \subseteq \mathbb{Z}^{n_{1}} \\
& y \in Y \subseteq \mathbb{R}^{n_{2}} .
\end{array}
$$

The idea of so-called decomposition algorithms is to rewrite the problem as

$$
\min \{h(x): x \in X \cap V\}
$$

where $V:=\left\{x \in \mathbb{Z}^{n_{1}}: \exists y \in Y: g(x, y) \leq 0\right\}$ is the projection of the feasible region on the integer variables and $h: \mathbb{Z}^{n_{1}} \rightarrow \mathbb{R}, h(x):=\min \{f(x, y): g(x, y) \leq 0, y \in Y\}$. We call this minimization problem, i.e., the remaining continuous problem if the integer variables are fixed in $(M I N L P)$, subproblem $(S(x))$ and assume that it is easy to solve. Two famous examples of decomposition algorithms are outer approximation and Benders decomposition which are explained in more detail in the following.

The idea of outer approximation (OA), first proposed by Duran and Grossmann [1986], is to construct a sequence of linearization points at which we construct supporting hyperplanes for $f$ and $g$ (we assume $f$ and $g$ to be continuously differentiable here) to approximate $h(x)$ and $V$. The original work by Duran and Grossmann [1986] was restricted to problems where the integer variables appear only linear. This restriction was removed by Fletcher and Leyffer [1994]. The algorithm is based on the observation that $(M I N L P)$ is equivalent to the master problem

$$
\begin{aligned}
\left(\text { Master }_{O A}\right) \min & \mu \\
\text { s.t. } \quad & \nabla f(\hat{x}, \hat{y})^{t}\left(\begin{array}{c}
x-\hat{x} \\
y-\hat{y}
\end{array}\right)+f(\hat{x}, \hat{y}) \leq \mu \forall(\hat{x}, \hat{y}) \in T, \\
& \nabla g(\hat{x}, \hat{y})^{t}\left(\begin{array}{c}
x-\hat{x} \\
y-\hat{y}
\end{array}\right)+g(\hat{x}, \hat{y}) \leq 0 \forall(\hat{x}, \hat{y}) \in T \cup S \\
& x \in X, y \in Y
\end{aligned}
$$

with $T:=\{(x, y): x \in X \cap V, y$ optimal to $(S(x))\}$ and $S:=\{(x, y): x \in X,(S(x))$ infeasible, $y$ solves $F(x)\}$ for the feasibility problem $F(x) \min \left\{\sum_{i=1}^{m} w_{i}: g(x, y) \leq w, y \in Y, w \geq 0\right\}$. 
The algorithm to solve $(M I N L P)$ consists of alternatingly solving a relaxed version of the master problem where $T$ and $S$ are approximated by $T^{i} \subseteq T$ and $S^{i} \subseteq S$ that contain not all linearization points and solving the subproblem to construct more pairs $(\hat{x}, \hat{y})$ to enlarge $T^{i}$ and $S^{i}$. It is shown in Duran and Grossmann [1986] and Fletcher and Leyffer [1994] respectively that the algorithm converges after a finite number of steps.

An implementation of a combined outer approximation and branch-and-bound approach is presented by Bonami et al. [2008].

Similar to outer approximation is the generalized Benders decomposition, introduced by Geoffrion [1972], generalizing the approach by Benders [1962] to mixed-integer convex problems. Here duality theory is used to derive a stepwise description of the function $h(x)$ and the projected feasible region $V$. Here the master problem is

$$
\begin{array}{ll}
(\text { Master Benders }) ~_{\text {min }} & \beta \\
\text { s.t. } & \inf _{y \in Y}\left\{f(x, y)+u^{t} g(x, y)\right\} \leq \beta \quad \forall u \geq 0 \\
& \inf _{y \in Y} \lambda^{t} g(x, y) \leq 0 \quad \forall \lambda \in\left\{\lambda \in \mathbb{R}^{m}: \lambda \geq 0, \sum_{i=1}^{m} \lambda_{i}=1\right\} \\
& x \in X .
\end{array}
$$

It is relaxed by ignoring most of the constraints and the algorithm consists of adding more and more constraints. To decide which constraints are added we solve again the subproblem $(S(x))$.

Comparing outer approximation and generalized Benders decomposition it is to say that the relaxed $\left(\right.$ Master $\left._{O A}\right)$ gives tighter bounds than the relaxed $\left(\right.$ Master $_{B e n d e r s}$ ) but on the other hand in each iteration of the OA algorithm a lot of constraints are added to the relaxed master problem while in the generalized Benders decomposition only one constraint is added. This means that after some iterations the relaxed $\left(\right.$ Master $\left._{O A}\right)$ is much more expensive to solve than the relaxed (Master Benders), compare e.g. Duran and Grossmann [1986], Fletcher and Leyffer [1994] or Grossmann [2002].

Contribution of this work As mentioned before, our approach is not dependent on $f$ and/or $g$ being convex or concave. In contrast, we impose assumptions on the geometric shape of the intersection of the feasible region and the level sets of the objective function. If these conditions hold, we get a straightforward algorithm to solve pure integer nonlinear optimization problems to optimality by solving its continuous relaxation and obtaining a finite candidate set: the set of all integer points we get by rounding each component of the continuous optimal solution either up or down. As any finite candidate set is a test set our results can be interpreted as the construction of a test set for special problems. On the other hand by proving that one of the rounded points of the continuous optimal solution $\bar{x}$ is an optimal solution $x^{*}$ to the integer problem we show that $\left\|\bar{x}-x^{*}\right\|_{\infty}<1$. This means, we give a proximity result: proximity results bound the distance between any optimal solution to the continuous relaxation to its closest optimal solution to the integer problem, compare e.g. Granot and Skorin-Kapov [1990] or Hochbaum and Shanthikumar [1990]. 


\subsection{Basic concepts and notations}

In this section we formally introduce the Rounding Property and its strengthening, the Strong Rounding Property. We give an intuition to our approach of investigating whether a given problem has the (Strong) Rounding Property: a level set approach. Furthermore, we provide some basic notations we use throughout this work.

\subsubsection{The Rounding Property}

To begin with, we show the basic idea in an easy example before we define and formalize what we need in the following.

Example 1.1. Consider the integer nonlinear problem

$$
\begin{array}{cl}
\min & \sin (x) \\
\text { s.t. } & 0 \leq x \leq 10 \\
& x \in \mathbb{Z} .
\end{array}
$$

This problem is neither convex nor concave, but of course it is easy to solve since there are only 10 feasible points. We compare the objective values of these points and get the unique optimal solution $x^{*}=5$. The unique optimal solution to the continuous relaxation $\min \{\sin (x): 0 \leq$ $x \leq 10\}$ is $\bar{x}=\frac{3 \pi}{2} \approx 4.71$, compare also Figure 1.1.

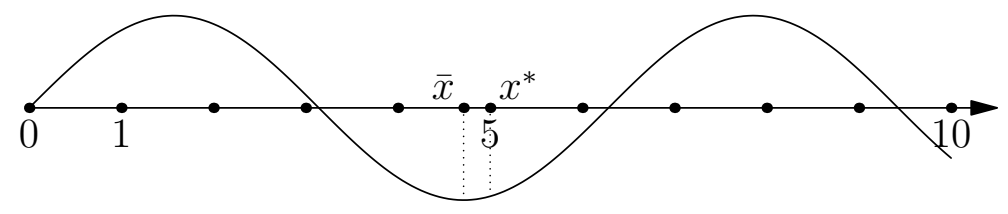

Figure 1.1.: The function $\sin (x)$ on $0 \leq x \leq 10$.

We see that this problem has the property that we can round the optimal solution to its continuous relaxation to get an optimal solution to the integer problem, even though the level sets are not Euclidean balls.

To formalize the above we introduce the following notation:

The problems we consider here are integer nonlinear optimization problems of the following form.

$$
\begin{array}{lll}
(I P) & \min & f(x) \\
\text { s.t. } & x \in F \\
& x \in \mathbb{Z}^{n}
\end{array}
$$

where $F \subseteq \mathbb{R}^{n}$, e.g. $F:=\left\{x \in \mathbb{R}^{n}: g_{i}(x) \leq 0 \forall i=1, \ldots, m\right\}$, and $f: F \rightarrow \mathbb{R}$. We denote this integer problem by the describing tuple $(f, F)$.

Notation 1.2. Given an integer optimization problem $(I P)$ we denote the set of optimal solutions by $X^{*}:=\left\{x \in \mathbb{Z}^{n} \cap F: f(x) \leq f(y) \forall y \in \mathbb{Z}^{n} \cap F\right\}$ and an optimal solution, i.e., an integer minimizer by $x^{*}$, if it exists. 
Basic Assumption 1.3. Throughout this work we always assume that an optimal solution to $(I P)$ exists, that is that $X^{*} \neq \emptyset$.

Assumption 1.3 justifies also that we wrote min instead of inf in $(I P)$.

As mentioned before, besides $(I P)$ we also consider its continuous relaxation

$$
\begin{array}{lll}
(C P) & \min & f(x) \\
\text { s.t. } & x \in F,
\end{array}
$$

where we skipped the integrality constraint $x \in \mathbb{Z}^{n}$.

Notation 1.4. We denote the set of optimal solutions to $(C P)$ by $\bar{X}:=\{x \in F: f(x) \leq$ $f(y) \forall y \in F\}$ and $a$ continuous minimizer by $\bar{x}$, if it exists.

The other important underlying assumption is the following:

Basic Assumption 1.5. We always assume that there exists an optimal solution to $(C P)$, i.e., $\bar{X} \neq \emptyset$ and that there exists an algorithm to solve $(C P)$ which we use as a black box.

The idea is that we only consider integer problems whose continuous relaxation is easier to solve, such that we would prefer to solve $(C P)$ instead of $(I P)$. An example for such a problem is an integer linear optimization problem, which is in general NP-hard whereas its continuous relaxation is solvable in polynomial time. This means that we assume that the difficulty in solving $(I P)$ comes from the integrality constraints.

Next we define what we mean by "rounding":

Notation 1.6. Given a point $x \in \mathbb{R}^{n}$ we denote the set of its rounded points by

$$
\operatorname{Round}(x):=\left\{y \in \mathbb{Z}^{n}: y_{i} \in\left\{\left\lfloor x_{i}\right\rfloor,\left\lceil x_{i}\right\rceil\right\} \forall i \in\{1, \ldots, n\}\right\}
$$

and its unique rounded point by

$$
\lfloor x\rceil:=\left\{y \in \mathbb{Z}^{n}: y_{i}=\left\lfloor x_{i}\right\rceil \forall i \in\{1, \ldots, n\}\right\}
$$

where $\lfloor a\rceil:=\operatorname{argmin}_{y \in \mathbb{Z}}|y-a|$, made unique by any fixed tie breaking rule, for $a \in \mathbb{R}$.

See Figure 1.2 for an example.

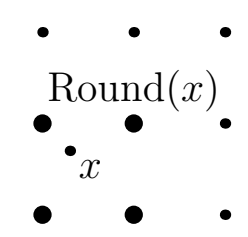

Figure 1.2.: Illustrating Notation 1.6: the bold points are Round(x) (left) and $\lfloor x\rceil$ (right).

We stress some important properties of the two defined objects: 
- $1 \leq|\operatorname{Round}(x)| \leq 2^{n}$ for every $x \in \mathbb{R}^{n}$ where $|\operatorname{Round}(x)|=1$ if and only if $x \in \mathbb{Z}^{n}$ and $|\operatorname{Round}(x)|=2^{n}$ if and only if $x_{i} \notin \mathbb{Z} \forall i=1, \ldots, n$.

- $\operatorname{Round}(x)=\left\{y \in \mathbb{Z}^{n}:\|x-y\|_{\infty}<1\right\}$.

- $\lfloor x\rceil$ is unique for every $x \in \mathbb{R}^{n}$. This is due to the fact that we break ties for example by the round-the-half-up rule. This might look like a useless complication, but we exploit explicitly this property that $\lfloor x\rceil$ is unique $\forall x \in \mathbb{R}^{n}$.

- $\lfloor x\rceil \in\left\{y \in \mathbb{Z}^{n}:\|x-y\|_{\infty} \leq 0.5\right\}$.

Using these definitions we can finally write down the basic property we examine in this work: the Rounding Property.

Definition 1.7. We say that an integer problem $(I P)$ has the Rounding Property $(R P)$ if for any optimal solution $\bar{x} \in \bar{X}$ to its continuous relaxation $(C P)$ there exists an optimal solution $x^{*} \in \operatorname{Round}(\bar{x})$.

This means that whichever optimal solution $\bar{x}$ to the continuous relaxation we take, $(I P)$ having the Rounding Property guarantees that $\operatorname{Round}(\bar{x}) \cap X^{*} \neq \emptyset$, so that it is enough to compare the (at most $2^{n}$ ) points in $\operatorname{Round}(\bar{x})$ to find an optimal solution to $(I P)$. Hence for every $\bar{x} \in \bar{X}$ the set $\operatorname{Round}(\bar{x})$ is a finite candidate set for $(I P)$. Alternatively, the Rounding Property can also be seen as a proximity result: as $\operatorname{Round}(\bar{x})=\left\{y \in \mathbb{Z}^{n}:\|\bar{x}-y\|_{\infty}<1\right\}$, $(I P)$ having the Rounding Property means that for every $\bar{x} \in \bar{X}$ there exists an $x^{*} \in X^{*}$ such that $\left\|\bar{x}-x^{*}\right\|_{\infty}<1$ (compare also Section 1.1).

Remark 1.8. We consider the minimizers to $(I P)$ and $(C P)$, so we do not know anything about $f\left(x^{*}\right)-f(\bar{x})$. This means also that we do not know whether $f(\bar{x})$ is a good lower bound on $f\left(x^{*}\right)$. We are interested in where the minimum is attained and not in its value. An example illustrating this difference can be found in Figure 2.14.

We illustrate this basic definition by some easy examples:

Example 1.9. In each of the following examples we consider the problem $\min \{f(x): x \in \mathbb{Z}\}$, where $f: \mathbb{R} \rightarrow \mathbb{R}$ as shown by the respective graph. As we are in a one-dimensional example we have $\operatorname{Round}(\bar{x})=\{\lfloor\bar{x}\rfloor,\lceil\bar{x}\rceil\}$.

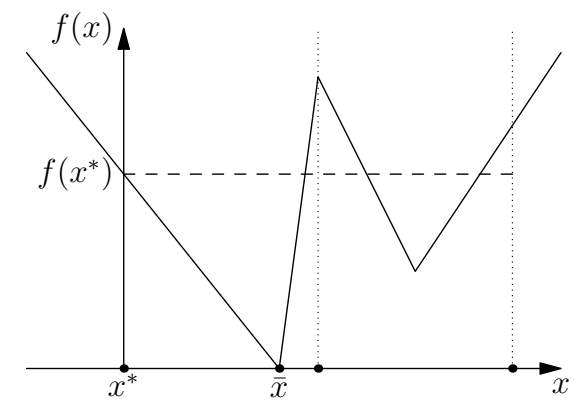

Figure 1.3.: (IP) has the Rounding Property.
In the first example the continuous as well as the integer minimizer are unique. Furthermore, we have

$$
x^{*} \in \operatorname{Round}(\bar{x})
$$

and therefore the RP. 


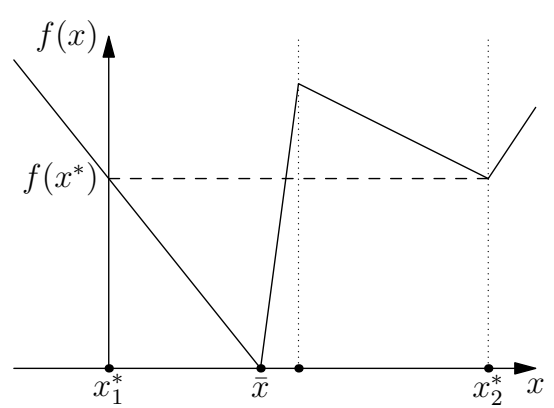

Figure 1.4.: $(I P)$ has the RP.

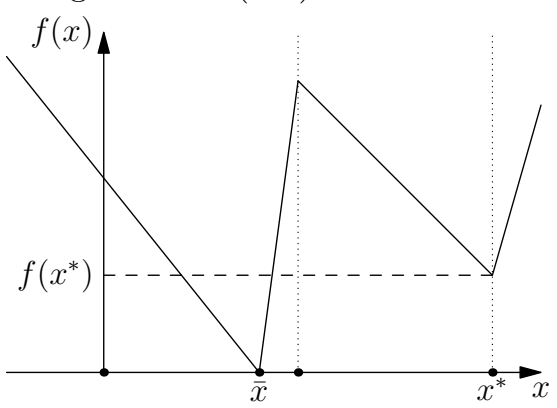

Figure 1.5.: $(I P)$ does not have the RP.

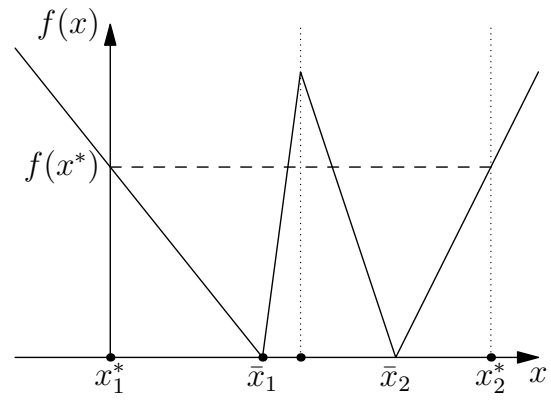

Figure 1.6.: (IP) has the RP.

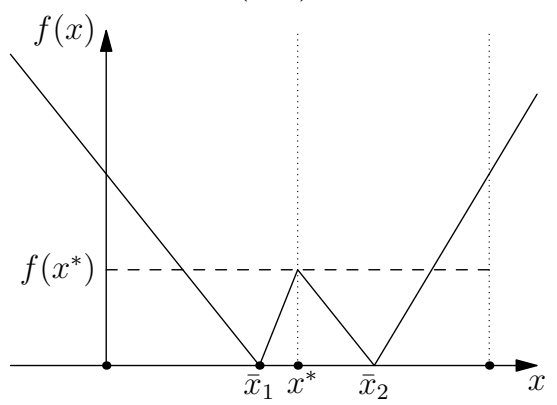

Figure 1.7.: $(I P)$ has the RP.
Again we have a unique continuous minimizer but this time two integer minimizers. Still we have the RP as

$$
X^{*} \cap \operatorname{Round}(\bar{x}) \neq \emptyset .
$$

Here the continuous as well as the integer minimizer are unique, but we do not have the RP, as

$$
x^{*} \notin \operatorname{Round}(\bar{x}) .
$$

In this example we have two continuous and two integer minimizers. As for each $\bar{x}_{i}$ there exists an $x_{j}^{*}$ such that

$$
x_{j}^{*} \in \operatorname{Round}\left(\bar{x}_{i}\right)
$$

we have the RP.

Also here $f$ has the RP: Now we have two continuous and only one integer minimizer, but still we have

$$
x^{*} \in \operatorname{Round}\left(\bar{x}_{1}\right)
$$

and

$$
x^{*} \in \operatorname{Round}\left(\bar{x}_{2}\right) .
$$




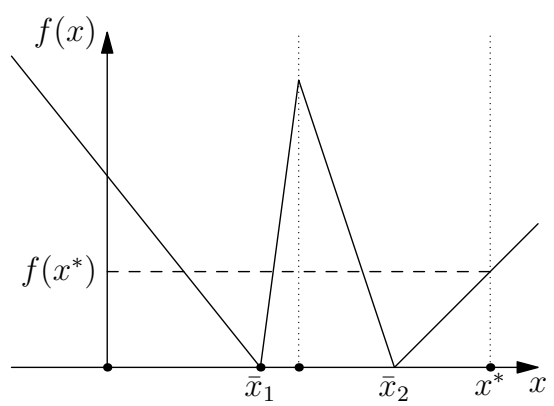

Figure 1.8.: (IP) does not have the RP.
Now we lost the RP: we still have two continuous minimizers and one integer minimizer, but while

$$
x^{*} \in \operatorname{Round}\left(\bar{x}_{2}\right)
$$

we have

$$
x^{*} \notin \operatorname{Round}\left(\bar{x}_{1}\right) .
$$

The Rounding Property provides us with the following straightforward algorithm to solve an integer problem that has the Rounding Property:

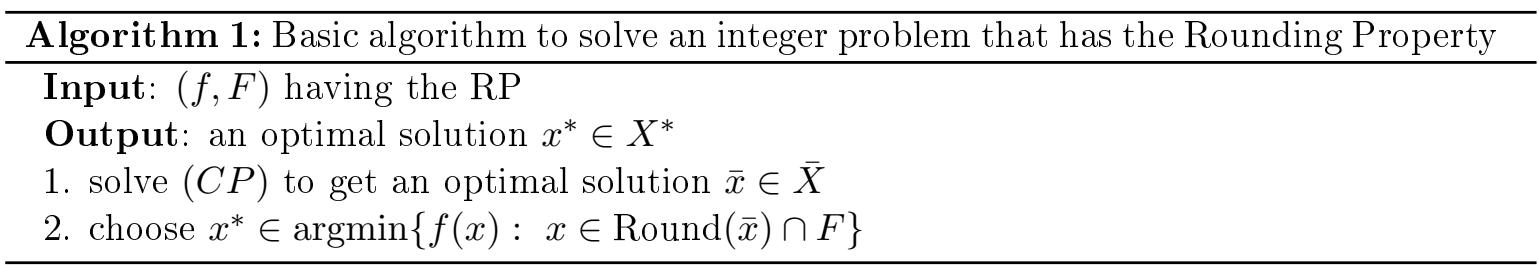

Lemma 1.10. Algorithm 1 is well-defined and correct, i.e.,

(i) $\operatorname{Round}(\bar{x}) \cap F \neq \emptyset$ and

(ii) $x^{*} \in X^{*}$.

Proof. As $(I P)$ has the RP there exists $\widetilde{x} \in X^{*} \cap \operatorname{Round}(\bar{x})$. This means,

(i) $\widetilde{x} \in X^{*} \cap \operatorname{Round}(\bar{x}) \subseteq F \cap \operatorname{Round}(\bar{x})$.

(ii) $f(\widetilde{x})=\min \{f(x): x \in F\}=\min \{f(x): x \in F \cap \operatorname{Round}(\bar{x})\}=f\left(x^{*}\right) \Rightarrow x^{*} \in X^{*}$.

The runtime of Algorithm 1 consists of the time to solve $(C P)$ and the evaluation of the objective function in up to $2^{n}$ points and their comparison. This means that its efficiency depends only on the efficiency in solving the continuous relaxation and the dimension $n$.

The latter point can be improved by observing that we do not have to take all points in Round $(\bar{x})$ into consideration, but only the feasible ones. Depending on the feasible region $F$ it is possible that $|\operatorname{Round}(\bar{x}) \cap F| \ll 2^{n}$.

One could think that it might be a little over the top to demand that for every optimal solution $\bar{x} \in \bar{X}$ we have $X^{*} \cap \operatorname{Round}(\bar{x}) \neq \emptyset$ - would it not be enough if there is one optimal solution to $(C P)$ that can be rounded? The problem is that if we need to find a particular optimal solution to $(C P)$ we cannot be sure that this could be done in the same time as solving $(C P)$, since finding an arbitrary solution is in general easier than finding a specific one. Furthermore, we do not know how to get a specific solution to $(C P)$ as we use a black box algorithm to solve it and we do not know which solution we are looking for, meaning that we would probably have to find all continuous optimal solutions, while most algorithms only find one. Compare also Chapter 4 for further remarks on this topic. 
If $n$ is rather large it might not be practical to evaluate and compare the objective values of all up to $2^{n}$ points in $\operatorname{Round}(\bar{x})$. Also if we think of the special case of binary optimization problems

$$
\begin{array}{lll}
(B P) \min & f(x) \\
\text { s.t. } & x \in F \\
& x \in\{0,1\}^{n},
\end{array}
$$

the Rounding Property would in general not be helpful since the continuous relaxation is $(C P) \min \left\{f(x): x \in F, x \in[0,1]^{n}\right\}$ and therefore $\bar{x}_{i} \in[0,1]$ for all $i=1, \ldots, n$ and $\operatorname{Round}(\bar{x})=\{0,1\}^{n}$ in the worst case. This means that here Algorithm 1 would need to enumerate all feasible 0 -1-vectors, which is always an (inefficient) way to solve $(B P)$.

In order to deal with both situations we introduce the Strong Rounding Property:

Definition 1.11. We say that an integer problem $(I P)$ has the Strong Rounding Property $(S R P)$ if for any optimal solution $\bar{x} \in \bar{X}$ to its continuous relaxation $(C P)$ it holds that $\lfloor\bar{x}\rceil \in X^{*}$.

The following relation between the Rounding and the Strong Rounding Property is obvious as $\lfloor x\rceil \in \operatorname{Round}(x)$ for all $x \in \mathbb{R}^{n}$.

Corollary 1.12. If (IP) has the Strong Rounding Property, it also has the Rounding Property.

Example 1.13. Let us consider again the problems in Example 1.9: the only one that has the SRP is the one in Figure 1.7, as $\left\lfloor\bar{x}_{1}\right\rceil=x^{*}=\left\lfloor\bar{x}_{2}\right\rceil$. This means that the opposite of Corollary 1.12 is of course not true in general.

The advantage of the Strong Rounding Property compared to the Rounding Property is that $\lfloor x\rceil$ is unique for all $x \in \mathbb{R}^{n}$, as we mentioned before. This means that as soon as we solved $(C P)$ we have a solution to $(I P)$ :

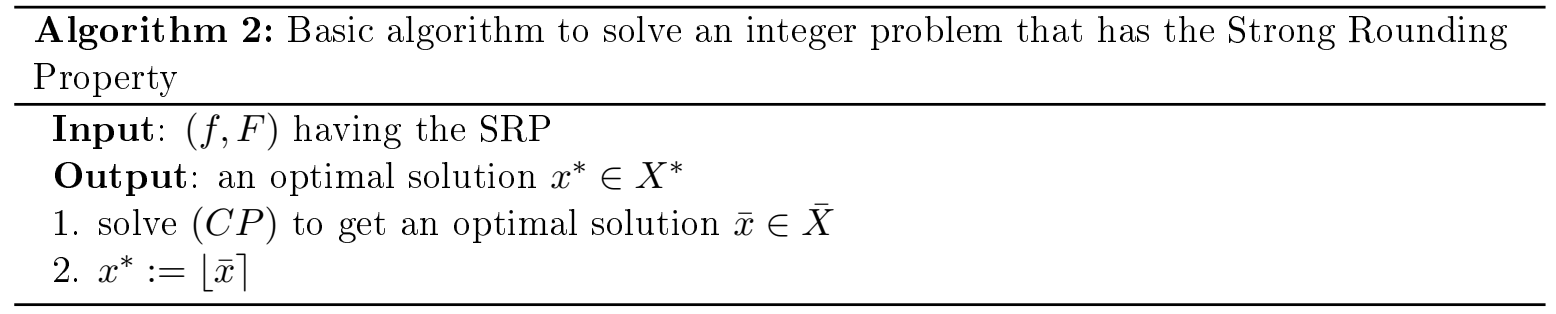

Corollary 1.14. Algorithm 2 is correct, i.e., $x^{*} \in X^{*}$.

Proof. This follows directly by the definition of the SRP.

The runtime of Algorithm 2 is the time we need to solve $(C P)$. In particular, if $(C P)$ is solvable in polynomial time so is $(I P)$ no matter what $n$ is.

But of course the Strong Rounding Property is a stricter assumption than the Rounding Property and therefore fulfilled for a smaller class of problems. Therefore we consider both properties in the following. 
Also the Strong Rounding Property can be seen as a proximity result:

$(f, F)$ has the SRP $\Rightarrow$ for all $\bar{x} \in \bar{X}$ there exists $x^{*} \in X^{*}$ such that $\left\|\bar{x}-x^{*}\right\|_{\infty} \leq 0.5$

But note that in contrast to the Rounding Property we do not get the reverse here. This is due to the fact that $\lfloor\bar{x}\rceil \in X^{*}$ is a stronger assumption than the existence of an $x^{*} \in X^{*}$ such that $\left\|\bar{x}-x^{*}\right\|_{\infty} \leq 0.5$, since we made $\lfloor\bar{x}\rceil$ unique.

Remark 1.15. We stress here that the Rounding Property as well as the Strong Rounding Property are trivially fulfilled as soon as $\bar{X} \subseteq \mathbb{Z}^{n}$. Accordingly, we can ignore all optimal solutions $\bar{x}$ to $(C P)$ that are already integer. Therefore we assume without loss of generality $\bar{X} \cap \mathbb{Z}^{n}=\emptyset$ in the following.

\subsubsection{Level set approach}

As mentioned before we investigate the level sets of the objective function to identify problems that have the (Strong) Rounding Property.

Notation 1.16. Given $F \subseteq \mathbb{R}^{n}$ and a function $f: F \rightarrow \mathbb{R}$ we call the set of all points whose objective value is less or equal a given level $z \in \mathbb{R}$ the level set of $f$ in $F$ corresponding to $z$ and denote it by

$$
\mathcal{L}_{\leq, f}^{F}(z):=\{x \in F: f(x) \leq z\} .
$$

Remark 1.17. - What we call a level set here is also often referred to as lower level set or sub level set.

- We write $\mathcal{L}_{\leq, f}(z)$ for $\mathcal{L}_{\leq, f}^{\mathbb{R}^{n}}(z)$.

- If there is no ambiguity we skip the subscript $f$.

- $\mathcal{L}_{\leq, f}(z)=\emptyset$ if and only if $z<\min _{x \in \mathbb{R}^{n}} f(x)$

Using the level sets of the objective function we can reformulate $(I P)$ (or $(C P)$ ) as

$$
\begin{array}{ll}
\min & z \\
\text { s.t. } & \mathcal{L}_{\leq, f}^{F}(z) \cap \mathbb{Z}^{n} \neq \emptyset \quad\left(\text { or } \mathcal{L}_{\leq, f}^{F}(z) \neq \emptyset\right) .
\end{array}
$$

(This reformulation and the proof can be found for example in Hamacher and Nickel [1995].)

Note that in this formulation we assume that $f$ is defined on $F$ which means that $\mathcal{L}_{\leq, f}^{F}(z)$ is the set of all feasible points whose objective value is smaller or equal than $z$. Therefore Problem (1.4) means finding the smallest level $z$ such there is at least one feasible point in the corresponding level set. This means that the optimal solution to (1.4) is the integer (or continuous) minimum of $(I P)$ whereas an optimal solution to $(I P)$ (or $(C P)$ ) is a minimizer. If we want to find a minimizer after solving (1.4) we can take any point in $\mathcal{L}_{\leq, f}^{F}\left(z^{*}\right) \cap \mathbb{Z}^{n}$ (or $\left.\mathcal{L}_{\leq, f}^{F}\left(z^{*}\right)\right)$.

We use this level set approach to decide whether a given integer problem has the Rounding Property. Therefore we use the following reformulation. 
Lemma 1.18. $\quad-(f, F)$ has the Rounding Property $\Longleftrightarrow$ for any optimal solution $\bar{x}$ to $(C P)$ and for all $x \in \mathbb{Z}^{n} \cap F$ we have that

$$
\mathcal{L}_{\leq}^{F}(f(x)) \cap \operatorname{Round}(\bar{x}) \neq \emptyset
$$

- $(f, F)$ has the Strong Rounding Property $\Longleftrightarrow$ for any optimal solution $\bar{x}$ to $(C P)$ and for all $x \in \mathbb{Z}^{n} \cap F$ we have that

$$
\lfloor\bar{x}\rceil \in \mathcal{L}_{\leq}^{F}(f(x)) .
$$

Proof. “”"

- Assume that $(f, F)$ has the RP and let $\bar{x}$ be an optimal solution to $(C P)$. Then there exists $x^{*} \in \operatorname{Round}(\bar{x})$ optimal to $(I P)$. This means that $x^{*} \in F$ and $f(x) \geq f\left(x^{*}\right)$ for any $x \in \mathbb{Z}^{n} \cap F$, hence $x^{*} \in \mathcal{L}_{\leq}(f(x))$ for all $x \in \mathbb{Z}^{n} \cap F$ and therefore $\mathcal{L}_{\leq}^{F}(f(x)) \cap \operatorname{Round}(\bar{x}) \neq$ $\emptyset$.

- If $(f, F)$ has the SRP, $x^{*}:=\lfloor\bar{x}\rceil$ is an optimal solution to $(I P)$ and hence contained in $\mathcal{L}_{\leq}^{F}(f(x))$ for all $x \in \mathbb{Z}^{n} \cap F$.

"£" Let $\bar{x}$ be optimal for $(C P)$. (Remember that we assumed that there is at least one optimal solution $x^{*}$ to $(I P)$, hence there is also at least one $x \in \mathbb{Z}^{n} \cap F$.)

- We know that $\mathcal{L}_{\leq}^{F}(f(x)) \cap \operatorname{Round}(\bar{x}) \neq \emptyset$ for any $x \in \mathbb{Z}^{n} \cap F$, i.e., for any $x \in \mathbb{Z}^{n} \cap F$ there exists a $y \in \operatorname{Round}(\bar{x}) \cap F$ such that $f(y) \leq f(x)$. This means, one of the points in $\operatorname{Round}(\bar{x}) \cap F$ is optimal for $(I P)$, and the RP holds.

- If $\lfloor\bar{x}\rceil \in \mathcal{L}_{\leq}(f(x))$ for any $x \in Z^{n} \cap F$ we obtain that $f(\lfloor\bar{x}\rceil) \leq f(x)$ for any $x \in \mathbb{Z}^{n} \cap F$, hence $\lfloor\bar{x}\rceil$ is optimal for $(I P)$, and the SRP holds.

We demonstrate the meaning of Lemma 1.18 in the following example.

Example 1.19. Consider the integer problem $\left(\|x-a\|_{2}, \mathbb{R}^{2}\right)$ where $a \in \mathbb{R}^{2}$. The level sets of the objective function $f: \mathbb{R}^{2} \rightarrow \mathbb{R}, x \mapsto\|x-a\|_{2}$ are circles centered at a, compare Figure 1.9.

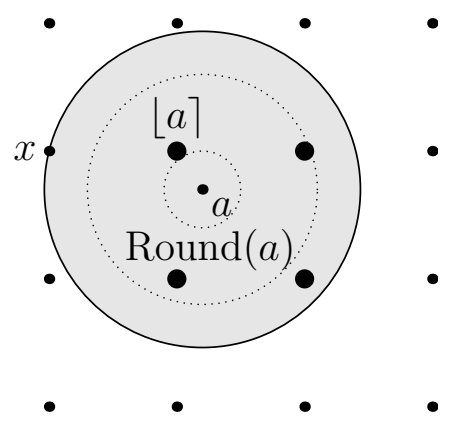

Figure 1.9.: $\left(\|x-a\|_{2}, \mathbb{R}^{2}\right)$ has the Strong Rounding Property. 
We see here that whenever a level set, i.e., a circle is large enough to contain an $x \in \mathbb{Z}^{2}$ it contains at least one point of $\operatorname{Round}(a)$. In fact we even have that $\lfloor a\rceil$ is contained in each such circle and hence $\left(\|x-a\|_{2}, \mathbb{R}^{2}\right)$ does not only have the RP but even the SRP.

We generalize the observation made in Example 1.19 to arbitrary dimensions and arbitrary $p$-norms.

Lemma 1.20. The integer problem $\left(\|x-a\|_{p}, \mathbb{R}^{n}\right)$ where $a \in \mathbb{R}^{n}$, has the Strong Rounding Property.

Proof. $\bar{x}=a$ is the unique optimal solution to the continuous relaxation. Furthermore, since $f(x)=\|x-a\|_{p}=\sqrt[p]{\sum_{i=1}^{n}\left|x_{i}-a_{i}\right|^{p}}$ and $\left|y-a_{i}\right| \geq\left|\left\lfloor a_{i}\right\rceil-a_{i}\right| \forall y \in \mathbb{Z}, \forall i=1, \ldots, n$ we get $f(\lfloor a\rceil) \leq f(x) \forall x \in \mathbb{Z}^{n}$.

This does not stay true if we use an arbitrary norm, for example $\|x\|_{Q}:=\sqrt{x^{t} Q x}$ for positive definite $Q \in \mathbb{R}^{n, n}$.

Example 1.21. Consider the problem $\left(\|x-a\|_{Q}, \mathbb{R}^{2}\right)$ where $a \in \mathbb{R}^{2}$ and $Q$ positive definite. Take for example $a=(0.4,0)$ and $Q=\left(\begin{array}{cc}909 & -525 \\ -525 & 349\end{array}\right)$. Then we get $\bar{x}=a$, $\operatorname{Round}(a)=\{(0,0),(1,0)\}$ and $f((0,0))=145.44, f((1,0))=327.24$ but $f((1,1))=46.24$. Hence $\operatorname{Round}(a) \cap X^{*}=\emptyset$, compare also Figure 1.10 .

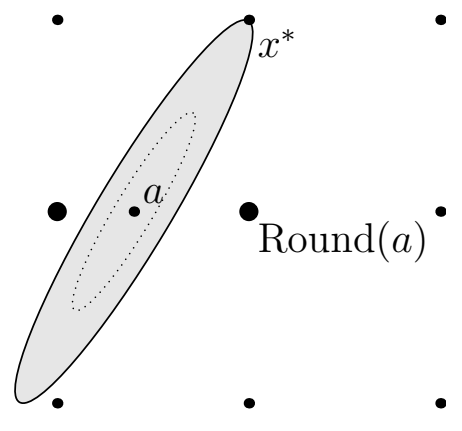

Figure 1.10.: $\left(\|x-a\|_{Q}, \mathbb{R}^{2}\right)$ does in general not have the Rounding Property.

We come back to the question under which assumption on $\|\cdot\|$ the problem $\left(\|x-a\|, \mathbb{R}^{n}\right)$ has the Rounding Property in Section 3.4.2. 


\subsection{Round sets}

We have seen in Lemma 1.20 that round level sets seem to comprise the Rounding Property. The purpose of this section is hence to generalize Lemma 1.20 to depend only on the shape of the level sets. But first we formalize what we mean by "round".

Notation 1.22. Let $p \in[1, \infty], x \in \mathbb{R}^{n}$ and $r \in \mathbb{R}_{0}^{+} \cup\{\infty\}$ then we denote by

$$
B_{p}(x, r):=\left\{y \in \mathbb{R}^{n}:\|x-y\|_{p} \leq r\right\}
$$

the scaled and shifted p-norm unit-ball.

For $r=\infty$ we have $B_{p}(x, r)=\mathbb{R}^{n}$ for all $p \in[1, \infty]$ and for all $x \in \mathbb{R}^{n}$.

Theorem 1.23 (Strong Rounding Property for $p$-norm balls). Let $(f, F)$ be given. Assume that for any optimal solution $\bar{x}$ to $(C P)$ we have that $\lfloor\bar{x}\rceil \in F$ and for any level $f(\bar{x}) \leq$ $z \leq f(\lfloor\bar{x}\rceil)$ there exists a $p \in[1, \infty]$ and a radius $r \in \mathbb{R}_{0}^{+} \cup\{\infty\}$ such that

$$
\mathcal{L}_{\leq}^{F}(z)=B_{p}(\bar{x}, r) .
$$

Then $(f, F)$ has the Strong Rounding Property.

Proof. Using Lemma 1.18 we show that for any optimal solution $\bar{x}$ to $(C P)$ and for all $x \in$ $\mathbb{Z}^{n} \cap F$ we have $\lfloor\bar{x}\rceil \in \mathcal{L}_{\leq}^{F}(f(x))$.

If $f(\lfloor\bar{x}\rceil) \leq f(x)$ we are done. Assume hence that $f(x) \leq f(\lfloor\bar{x}\rceil)$. This means, there exist $p \in[1, \infty]$ and $r \in \mathbb{R}_{0}^{+} \cup\{\infty\}$ such that $\mathcal{L}_{\leq}^{F}(f(x))=B_{p}(\bar{x}, r)$. As $x \in F$ we have $x \in \mathcal{L}_{\leq}^{F}(f(x))$ and therefore $r \geq\|\bar{x}-x\|_{p}$.

$$
\begin{aligned}
\Rightarrow \quad\|\bar{x}-\lfloor\bar{x}\rceil\|_{p} & \leq\|\bar{x}-x\|_{p} \quad \text { as } x \in \mathbb{Z}^{n} \\
& \leq r
\end{aligned}
$$

This means that $\lfloor\bar{x}\rceil \in B_{p}(\bar{x}, r)=\mathcal{L}_{\leq}^{F}(f(x))$.

Along with Theorem 1.23 we state that the assumptions of this theorem lead to a unique $\bar{x}$.

Lemma 1.24. Assume that for any optimal solution $\bar{x}$ to $(C P)$ there exists a $p(\bar{x}) \in[1, \infty]$ and a radius $r(\bar{x}) \in \mathbb{R}_{0}^{+} \cup\{\infty\}$ such that $\bar{X}=B_{p}(\bar{x}, r)$. Then we have $|\bar{X}|=1$ or $\bar{X}=\mathbb{R}^{n}$.

Proof. Assume that $\bar{X} \neq \mathbb{R}^{n}$. By our basic assumption we know that $\bar{X} \neq \emptyset$. Let $\bar{x}_{1} \in \bar{X}$. Assume that $\bar{X} \neq\left\{\bar{x}_{1}\right\}$, i.e., $0<r\left(\bar{x}_{1}\right)<\infty$. Let $x_{2} \in B_{p\left(\bar{x}_{1}\right)}\left(\bar{x}_{1}, r\left(\bar{x}_{1}\right)\right)$, such that $\| x_{2}-$ $\bar{x}_{1} \|_{p\left(\bar{x}_{1}\right)}=r\left(\bar{x}_{1}\right)$. This means, $x_{2} \neq \bar{x}_{1}$ and $x_{2} \in \bar{X}$. Thus there exist $p\left(x_{2}\right) \in[1, \infty]$ and $r\left(x_{2}\right) \in \mathbb{R}^{+}$such that $\bar{X}=B_{p\left(x_{2}\right)}\left(x_{2}, r\left(x_{2}\right)\right) .\left(r\left(x_{2}\right)>0\right.$ as $\bar{x}_{1} \in \bar{X}$ and $\left.\bar{x}_{1} \neq x_{2}.\right)$ Then we know that $r\left(x_{2}\right) \geq\left\|\bar{x}_{1}-x_{2}\right\|_{p\left(x_{2}\right)}$ as $\bar{x}_{1} \in \bar{X}$.

For $\bar{x}_{3}=2 x_{2}-\bar{x}_{1}$ we have $x_{3} \in B_{p\left(x_{2}\right)}\left(x_{2}, r\left(x_{2}\right)\right)=\bar{X}$ since $\left\|x_{3}-x_{2}\right\|_{p\left(x_{2}\right)}=\left\|\bar{x}_{1}-x_{2}\right\|_{p\left(x_{2}\right)} \leq$ $r\left(x_{2}\right)$, but also

$$
\left\|x_{3}-\bar{x}_{1}\right\|_{p\left(\bar{x}_{1}\right)}=2\left\|x_{2}-\bar{x}_{1}\right\|_{p\left(\bar{x}_{1}\right)}>\left\|x_{2}-\bar{x}_{1}\right\|_{p\left(\bar{x}_{1}\right)}=r\left(\bar{x}_{1}\right) \quad \text { since } x_{2} \neq \bar{x}_{1}
$$

which is a contradiction to $\bar{X}=B_{p\left(\bar{x}_{1}\right)}\left(\bar{x}_{1}, r\left(\bar{x}_{1}\right)\right)$. (See also Figure 1.11 for an illustration.) 


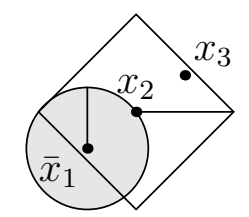

Figure 1.11.: Illustrating the proof of Lemma 1.24.

Theorem 1.23 is a basic scheme we will see often in this work. Therefore, we add a few comments here. The basic form of this type of theorems is always the same:

Let $(f, F)$ be given. Assume that for any optimal solution $\bar{x}$ to $(C P)$ and for any level $f(\bar{x}) \leq z \leq \min \{f(x): x \in \operatorname{Round}(\bar{x}) \cap F\} \quad$ or $f(\bar{x}) \leq z \leq f(\lfloor\bar{x}\rceil)$ respectively) the level set $\mathcal{L}_{<}^{F}(z)$ has the geometric shape $\mathcal{A}$ with respect to $\bar{x}$. Then $(f, F)$ has the (Strong) Rounding Property.

The basic idea here is as long as the level sets spread nicely, i.e., they have shape $\mathcal{A}$ (in Theorem 1.23 that would be scaled and shifted $p$-norm balls w.r.t. $\bar{x}$ ), for small $z$, we can be sure that the first integer point reached by the growing level sets will be one of the rounded ones. We only need this nice behavior until the first integer point is touched. But as we do not know which one will be the first one, we demand it until the first of the rounded points is reached.

We have seen in the proof of Theorem 1.23 that we actually only need this nice shape for the level sets that contain a feasible integer point on their boundary but we write our theorems with the stricter condition that it holds for all $z \leq f(\lfloor\bar{x}\rceil)$ because we assume that this is easier to test, compare Chapter 4 for a relaxation.

Especially to Theorem 1.23 we make the following comments.

(i) Note that we allow that $p$ and $r$ might differ for different levels (and also for different $\bar{x}$ but we have seen in Lemma 1.24 that $\bar{x}$ is unique), see Figure 1.12 for an example: the level sets displayed here are scaled and shifted $p$-norm balls, until the first integer point is reached - thus the conditions of Theorem 1.23 are met.

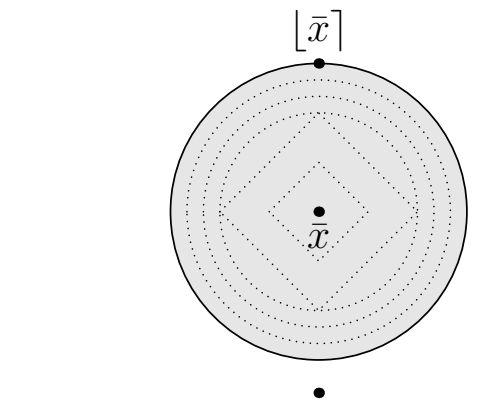

Figure 1.12.: Example for the first level sets of a function that lead to the Strong Rounding Property due to Theorem 1.23. 
(ii) Note also the difference between the statement in Lemma 1.20 and the one in Theorem 1.23: while we assumed in Lemma 1.20 that the function is a shifted $p$-norm, we only assume that the level sets have the shape of scaled and shifted $p$-norm balls in Theorem 1.23. This condition is for example also met by the following function:

$$
f(x)= \begin{cases}0 & \text { for } x=a \\ 1 & \text { for } x:\|x-a\|_{p} \leq 1 \\ 2 & \text { for } x:\|x-a\|_{p} \leq 2 \\ \|x-a\|_{p} & \text { otherwise. }\end{cases}
$$

Furthermore, we allow in Theorem 1.23 different $p$-norm balls for each level.

(iii) The theorem is not true in general if we replace $p$-norm balls by arbitrary norm balls: compare the example displayed in Figure 1.13: the set $B$ defines a norm by $\gamma_{B}(x):=$ $\inf \{\lambda \geq 0: x \in \lambda \cdot B\}$ since $B$ is convex, compact, centrally symmetric and contains the origin in its interior. The level sets displayed can therefore be seen as the level sets of $\gamma_{B}(x-\bar{x})$. Nevertheless, it is obvious that $x^{*} \notin \operatorname{Round}(\bar{x})$ so that $\left(\gamma_{B}, \mathbb{R}^{2}\right)$ does not even have the RP.

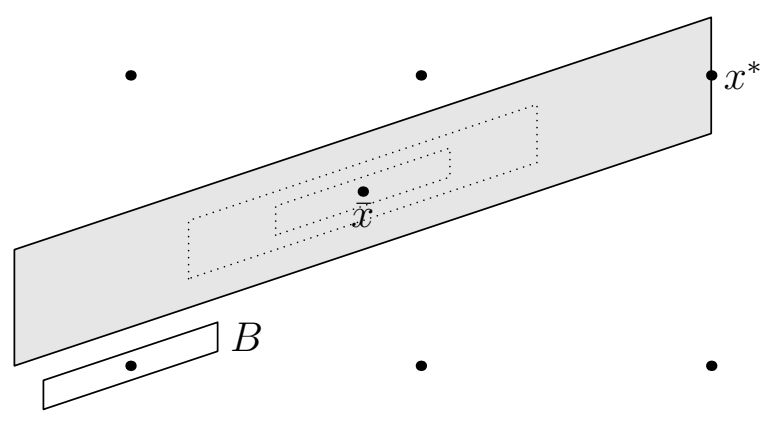

Figure 1.13.: Example that Theorem 1.23 does not stay true if the inducing norm is not a $p$-norm but an arbitrary norm.

(iv) Let us consider the special case $n=1$. Here $B_{p}(x, r)=\{y:|x-y| \leq r\}$ for all $p \in[1, \infty]$.

This can easily be generalized to guarantee the Rounding Property instead of the Strong Rounding Property: the level sets do not need to be symmetric intervals about $\bar{x}$ but can be arbitrary nested intervals.

Lemma 1.25. Let $n=1$ and $(f, F)$ be given. Assume that for any optimal solution $\bar{x}$ to $(C P)$ and for any level $f(\bar{x}) \leq z \leq \min \{f(x): x \in \operatorname{Round}(\bar{x}) \cap F\}$ the level set $\mathcal{L}_{\leq}^{F}(z)$ is an interval. Then $(f, F)$ has the Rounding Property. If $\lfloor\bar{x}\rceil \in F$ and the intervals are symmetric about $\bar{x}$ for all $f(\bar{x}) \leq z \leq f(\lfloor\bar{x}\rceil)$, then $(f, F)$ has the Strong Rounding Property.

Proof. (i) Let $\bar{x} \in \bar{X}$ and $x \in \mathbb{Z}^{n} \cap F$. Using Lemma 1.18 we show $\mathcal{L}_{\leq, f}^{F}(f(x)) \cap \operatorname{Round}(\bar{x}) \neq$ $\emptyset$. If $\min \{f(u): u \in \operatorname{Round}(\bar{x}) \cap F\} \leq f(x)$ we are done. Otherwise we have 
that $\mathcal{L}_{\leq}^{F}(f(x))$ is connected. This means that $\mathcal{L}_{\leq}^{F}(f(x)) \supseteq\left\{\begin{array}{ll}{[\bar{x}, x]} & \text { if } \bar{x} \leq x \\ {[x, \bar{x}]} & \text { if } x<\bar{x} .\end{array}\right.$ Define $\widetilde{x}:=\left\{\begin{array}{ll}\lceil\bar{x}\rceil & \text { if } \bar{x} \leq x \\ \lfloor\bar{x}\rfloor & \text { if } x \leq \bar{x} .\end{array}\right.$ Then we have $\widetilde{x} \in \operatorname{Round}(\bar{x})$ and $\widetilde{x} \in \mathcal{L}_{\leq}^{F}(f(x))$, since $\widetilde{x} \in$ $\left\{\begin{array}{ll}{[\bar{x}, x]} & \text { if } \bar{x} \leq x \\ {[x, \bar{x}]} & \text { if } x<\bar{x}\end{array}\right.$. This means, $\mathcal{L}_{\leq}^{F}(f(x)) \cap \operatorname{Round}(\bar{x}) \neq \emptyset$.

(ii) If the level sets are symmetric intervals about $\bar{x}$, for each $z \leq f(\lfloor\bar{x}\rceil)$ there exists a radius $r(z)$ such that $\mathcal{L}_{\leq}^{F}(z)=\{x \in \mathbb{R}:|x-\bar{x}| \leq r\}$. This means that either $\bar{X}=\mathbb{R}$ which implies the Strong Rounding Property or $\bar{X}=\{\bar{x}\}$, compare Lemma 1.24. The latter case means that the assumptions of Theorem 1.23 are fulfilled (for any $p \in[1, \infty]$ ) and hence the problem has the Strong Rounding Property.

In the following we will see which properties of $B_{p}(\bar{x}, r)$ do guarantee the (Strong) Rounding Property and use these insights to find more general shapes that lead to either the Rounding Property or the Strong Rounding Property. 


\section{Part I.}

\section{Sufficient conditions for the Rounding Property}





\section{Quasi-round sets}

Theorem 1.23 shows that "round level sets", i.e., level sets that have the shape of scaled $p$ norm-balls, lead to the Strong Rounding Property. This raises the question: what if the sets are not "totally round" but only "almost round", do we at least keep the Rounding Property?

The remainder of this chapter is organized as follows. First we introduce the concept of quasi-round sets and show some basic properties. Then we show for which "degree of quasiroundness" we get the Rounding or even the Strong Rounding Property. In the next section we show some examples of functions whose level sets are quasi-round as well as an application to robust optimization. We end this chapter by a generalization that leads to proximity results.

\subsection{Properties of quasi-round sets}

The basic concept of this chapter is the quasi-roundness of a set.

Definition 2.1. Given $\alpha \geq 0$ and $p \in[1, \infty]$ we call a set $M \subseteq \mathbb{R}^{n}(\alpha, p)$-quasi-round with respect to a point $x^{0}$ if there exist $r \in \mathbb{R}_{0}^{+}$and $R \in \mathbb{R}_{0}^{+}$where $R-r \leq \alpha$ such that

$$
B_{p}\left(x^{0}, r\right) \subseteq M \subseteq B_{p}\left(x^{0}, R\right) .
$$

See some examples of $(0.4,2)$-quasi-round sets in Figure 2.1.
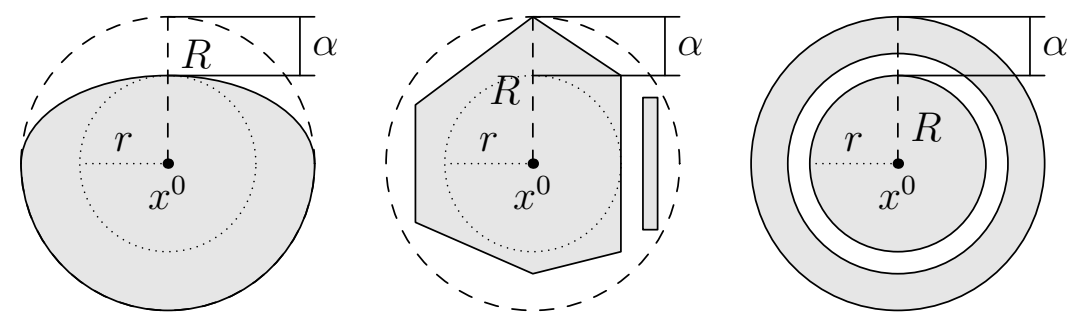

Figure 2.1.: Examples for sets that are $(0.4,2)$-quasi-round.

Whether a given set $M$ is $(\alpha, p)$-quasi-round depends on $\alpha$ and $p$, see Figure 2.2. 


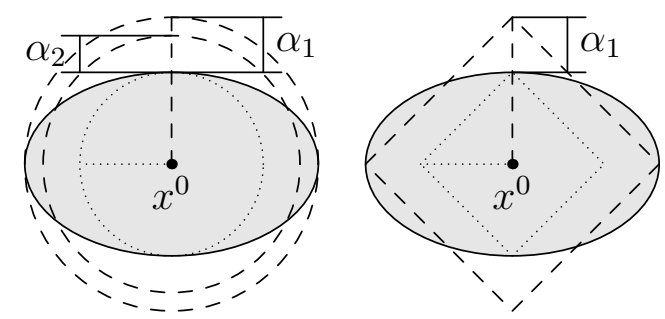

Figure 2.2.: The ellipsoid is $\left(\alpha_{1}, 2\right)$-quasi-round, but not $\left(\alpha_{2}, 2\right)$-quasi-round nor $\left(\alpha_{1}, 1\right)$-quasiround.

First of all we observe that $p$-round sets are $(\alpha, p)$-quasi-round for every $\alpha$ and that on the other hand the only sets that are $(0, p)$-quasi-round w.r.t. $x^{0}$ are sets of the form $B_{p}\left(x^{0}, r\right)$ for $r \geq 0$.

Lemma 2.2. $M \subseteq \mathbb{R}^{n}$ is $(\alpha, p)$-quasi-round w.r.t. $x^{0}$ for every $\alpha \geq 0$ if and only if $M=$ $B_{p}\left(x^{0}, r\right)$.

Proof. " $\Rightarrow$ " If $M$ is $(0, p)$-quasi-round w.r.t. $x^{0}$ there exists $r \in \mathbb{R}_{0}^{+}$such that $B_{p}\left(x^{0}, r\right) \subseteq$ $M \subseteq B_{p}\left(x^{0}, r\right)$.

" $\Leftarrow " M=B_{p}\left(x^{0}, r\right)$ is $(\alpha, p)$-quasi-round w.r.t. $x^{0}$ for every $\alpha \geq 0$ : set $R=r$.

Next we state some direct consequences of Definition 2.1.

Remark 2.3. Let $M \subseteq \mathbb{R}^{n}$ be $(\alpha, p)$-quasi-round w.r.t. $x^{0}$ and let $r, R \in \mathbb{R}_{0}^{+}$such that $B_{p}\left(x^{0}, r\right) \subseteq M \subseteq B_{p}\left(x^{0}, R\right)$. Then we observe

(i) $R \geq r$ as $B_{p}\left(x^{0}, r\right) \subseteq B_{p}\left(x^{0}, R\right)$.

(ii) $M$ is bounded, as an unbounded set can never be $(\alpha, p)$-quasi-round for any $\alpha$ and any $p \in[1, \infty]$ since we require $R<\infty$.

(iii) $r \geq 0$ means that $x^{0}$ needs to be in $M$. This is not necessary, but in the following we investigate whether level sets are quasi-round w.r.t. a continuous minimizer and therefore we have $x^{0} \in M$ anyway.

(iv) $M$ is also $\left(\alpha^{\prime}, p\right)$-quasi-round w.r.t. $x^{0}$ for all $\alpha^{\prime} \geq \alpha$.

In the next lemma we identify some set manipulations that preserve the quasi-roundness of sets.

Lemma 2.4. Let $M \subseteq \mathbb{R}^{n}$ be $(\alpha, p)$-quasi-round w.r.t. $x^{0} \in M$. Then the following holds:

(i) $M+x$ is $(\alpha, p)$-quasi-round w.r.t. $x^{0}+x$ for all $x \in \mathbb{R}^{n}$.

(ii) Any reflection of $M$ about a hyperplane through $x^{0}$ parallel to the coordinate axes is $(\alpha, p)$-quasi-round w.r.t. $x^{0}$. 
Let $M_{1} \subseteq \mathbb{R}^{n}$ be $\left(\alpha_{1}, p\right)$-quasi-round w.r.t. $x^{1} \in M_{1}$ and $M_{2} \subseteq \mathbb{R}^{n}\left(\alpha_{2}, p\right)$-quasi-round w.r.t. $x^{2} \in M_{2}$. Let $R_{1}:=\min \left\{R \in \mathbb{R}: M_{1} \subseteq B_{p}\left(x^{1}, R\right)\right\}$ and $R_{2}:=\min \left\{R \in \mathbb{R}: M_{2} \subseteq\right.$ $\left.B_{p}\left(x^{2}, R\right)\right\}$. Let w.l.o.g. $R_{1} \geq R_{2}$. Then the following holds:

(iii) If $x^{1}=x^{2}$ the union $M_{1} \cup M_{2}$ is $\left(\alpha_{1}, p\right)$-quasi-round w.r.t. $x^{1}$.

(iv) If $x^{1}=x^{2}$ and $R_{1}-\alpha_{1}<R_{2}-\alpha_{2}$ the intersection $M_{1} \cap M_{2}$ is $\left(\alpha_{1}, p\right)$-quasi-round w.r.t. $x^{1}$. If $x^{1}=x^{2}$ and $R_{2}-\alpha_{2} \leq R_{1}-\alpha_{1}$ then $M_{1} \cap M_{2}$ is $\left(\alpha_{2}, p\right)$-quasi-round w.r.t. $x^{1}$.

(v) The Minkowski sum $M_{1}+M_{2}:=\left\{m_{1}+m_{2}: m_{1} \in M_{1}, m_{2} \in M_{2}\right\}$ is $\left(\alpha_{1}+\alpha_{2}, p\right)$-quasiround w.r.t. $x^{1}+x^{2}$.

Proof. (i) This is a special case of (v) since $\{x\}$ is $(0, p)$-quasi-round w.r.t. $x$ for all $p \in$ $[1, \infty]$.

(ii) Reflecting the set about hyperplanes through $x^{0}$ parallel to the coordinate axes does not change the distances of the points in $M$ to $x^{0}$.

(iii) $M_{1} \cup M_{2} \subseteq B_{p}\left(x^{1}, R_{1}\right)$ since $M_{2} \subseteq B_{p}\left(x^{1}, R_{2}\right) \subseteq B_{p}\left(x^{1}, R_{1}\right)$. And since $B_{p}\left(x^{1}, r_{1}\right) \subseteq M_{1}$ we have $B_{p}\left(x^{1}, r_{1}\right) \subseteq M_{1} \cup M_{2} \subseteq B_{p}\left(x^{1}, R_{1}\right)$ and $R_{1}-r_{1} \leq \alpha_{1}$.

(iv) $M_{1} \cap M_{2} \subseteq B_{p}\left(x^{1}, R_{2}\right) \subseteq B_{p}\left(x^{1}, R_{1}\right)$. Let $r_{1}:=R_{1}-\alpha_{1}$. This means, $B_{p}\left(x^{1}, r_{1}\right) \subseteq M_{1}$ by definition of $R_{1}$ since $M_{1}$ is $\left(\alpha_{1}, p\right)$-quasi-round w.r.t. $x^{1}$. Let $r_{2}:=R_{2}-\alpha_{2}$. Then we get by the same argument $B_{p}\left(x^{1}, r_{2}\right) \subseteq M_{2}$.

If $r_{1}<r_{2}$ we have $B_{p}\left(x^{1}, r_{1}\right) \subseteq B_{p}\left(x^{1}, r_{2}\right) \subseteq M_{2}$ and hence $B_{p}\left(x^{1}, r_{1}\right) \subseteq M_{1} \cap M_{2} \subseteq$ $B_{p}\left(x^{1}, R_{1}\right)$ and $R_{1}-r_{1} \leq \alpha_{1}$.

If $r_{1} \geq r_{2}$ we get analogously $B_{p}\left(x^{1}, r_{2}\right) \subseteq M_{1} \cap M_{2} \subseteq B_{p}\left(x^{1}, R_{2}\right)$ and $R_{2}-r_{2} \leq \alpha_{2}$.

(v) $M_{1}+M_{2} \subseteq B_{p}\left(x^{1}+x^{2}, R_{1}+R_{2}\right)$ :

Let $m \in M_{1}+M_{2}$, i.e., $m=m_{1}+m_{2}$ for some $m_{1} \in M_{1}$ and $m_{2} \in M_{2}$. This means, $\left\|m-\left(x^{1}+x^{2}\right)\right\|_{p} \leq\left\|m_{1}-x^{1}\right\|_{p}+\left\|m_{2}-x^{2}\right\|_{p} \leq R_{1}+R_{2}$. Furthermore,

$$
B_{p}\left(x^{1}+x^{2}, r_{1}+r_{2}\right)=\underbrace{B_{p}\left(x^{1}, r_{1}\right)}_{\subseteq M_{1}}+\underbrace{B_{p}\left(x^{2}, r_{2}\right)}_{\subseteq M_{2}} \subseteq M_{1}+M_{2}
$$

and $R_{1}+R_{2}-\left(r_{1}+r_{2}\right) \leq \alpha_{1}+\alpha_{2}$. 


\subsection{Quasi-round sets and the Rounding Property}

In this section we investigate how to use quasi-round level sets to derive the Rounding Property. As we have seen that $(0, p)$-quasi-round sets are exactly the scaled $p$-norm-balls and on the other hand if $\alpha$ is large enough every bounded set is $(\alpha, p)$-quasi-round, it is obvious that the question whether quasi-round level sets lead to the Rounding Property is going to depend highly on $\alpha$.

Theorem 2.5 (Rounding Property for quasi-round level sets). Let $(f, F)$ be given. Assume that for any optimal solution $\bar{x}$ to $(C P)$ and for any level $f(\bar{x}) \leq z \leq \min \{f(x): x \in$ $\operatorname{Round}(\bar{x}) \cap F\}$ there exists $p \in[1, \infty]$ such that the level set $\mathcal{L}_{\leq}^{F}(z)$ is $\left(\alpha_{p}(\bar{x}, F), p\right)$-quasiround with respect to $\bar{x}$, where

$$
\alpha_{p}(\bar{x}, F):=d_{p}\left(\bar{x},\left(\mathbb{Z}^{n} \cap F\right) \backslash \operatorname{Round}(\bar{x})\right)-d_{p}\left(\bar{x}, \mathbb{Z}^{n} \cap F\right) .
$$

Then $(f, F)$ has the Rounding Property.

Here $d_{p}(x, M):=\min _{y \in M}\|x-y\|_{p}$ is the $p$-norm point-set distance. Note that $\alpha_{p}(\bar{x}, F) \geq 0$, hence $\left(\alpha_{p}(\bar{x}, F), p\right)$-quasi-roundness is well-defined.

Proof. Let $\bar{x} \in \bar{X}$ and $x \in \mathbb{Z}^{n} \cap F$. Using Lemma 1.18 we show that $\mathcal{L}_{\leq}^{F}(f(x)) \cap \operatorname{Round}(\bar{x}) \neq \emptyset$. If $\min \{f(u): u \in \operatorname{Round}(\bar{x}) \cap F\} \leq f(x)$ we are done. Otherwise we have that $\mathcal{L}_{<}^{F}(f(x))$ is $\left(\alpha_{p}(\bar{x}, F), p\right)$-quasi-round w.r.t. $\bar{x}$ and hence there exist $r, R \in \mathbb{R}_{0}^{+}$such that $B_{p}(\bar{x}, r) \subseteq$ $\mathcal{L}_{<}^{F}(f(x)) \subseteq B_{p}(\bar{x}, R)$ and $R-r \leq \alpha_{p}(\bar{x}, F)$.

As $d_{p}\left(\bar{x}, \mathbb{Z}^{n} \cap F\right) \geq d_{p}\left(\bar{x}, \mathbb{Z}^{n}\right)=\|\bar{x}-\lfloor\bar{x}\rceil\|_{p}$, compare Lemma 1.20, we have

$$
\begin{aligned}
\|\bar{x}-\lfloor\bar{x}\rceil\|_{p} & \leq d_{p}\left(\bar{x},\left(\mathbb{Z}^{n} \cap F\right) \backslash \operatorname{Round}(\bar{x})\right)-\alpha_{p}(\bar{x}, F) \\
& \leq\|\bar{x}-x\|_{p}-\alpha_{p}(\bar{x}, F) \quad \text { as } x \in \mathbb{Z}^{n} \cap F \text { and } x \notin \operatorname{Round}(\bar{x}) \\
& \leq R-\alpha_{p}(\bar{x}, F) \quad \text { as } x \in \mathcal{L}_{\leq}^{F}(f(x)) \subseteq B_{p}(\bar{x}, R) \\
& \leq r .
\end{aligned}
$$

This means, $\lfloor\bar{x}\rceil \in B_{p}(\bar{x}, r) \subseteq \mathcal{L}_{\leq}^{F}(f(x))$ and hence Round $(\bar{x}) \cap F \neq \emptyset$. Thus there exists $\widetilde{x} \in \operatorname{argmin}\{f(u): u \in \operatorname{Round}(\bar{x}) \cap F\}$ and $f(\widetilde{x}) \leq f(\lfloor\bar{x}\rceil) \leq f(x)<f(\widetilde{x})$.

Like this $f(x)<\min \{f(u): u \in \operatorname{Round}(\bar{x}) \cap F\}$ leads to a contradiction.

Notation 2.6. Throughout this chapter we denote by $\widetilde{x}$ an element of $\operatorname{argmin} d_{p}\left(\bar{x}, \mathbb{Z}^{n} \backslash\right.$ $\operatorname{Round}(\bar{x}))$ and by $\widetilde{x}^{F}$ an element of $\operatorname{argmin} d_{p}\left(\bar{x},\left(\mathbb{Z}^{n} \cap F\right) \backslash \operatorname{Round}(\bar{x})\right)$. These are the closest, not rounded, (feasible) points. Analogously we denote by $\lfloor\bar{x}\rceil^{F}$ an element of $\operatorname{argmin} d_{p}\left(\bar{x}, \mathbb{Z}^{n} \cap\right.$ $F)$, i.e., a closest feasible integer.

Remark 2.7. (i) Note that also here, like in Theorem 1.23, it does not have to be the same $p$ for all levels $z$, but it can depend on $z$.

(ii) The choice of $\alpha_{p}(\bar{x}, F)$ makes sure that as soon as a feasible integer point is in one of the level sets (and therefore in $B_{p}(\bar{x}, R)$ ), $r$ is large enough such that there is at least one feasible rounded point in $B_{p}(\bar{x}, r)$ and therefore also in the level set, see also Figure 2.3. 


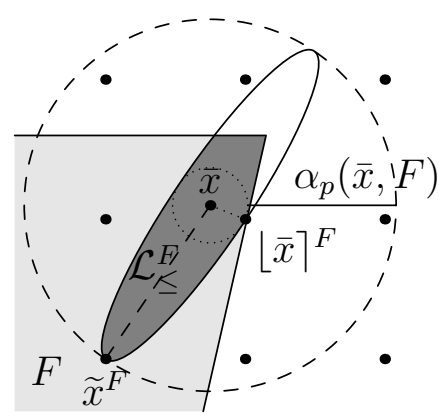

Figure 2.3.: Illustrating the definition of $\alpha_{p}(\bar{x}, F):\lfloor\bar{x}\rceil^{F} \in \operatorname{argmin} d_{2}\left(\bar{x}, \mathbb{Z}^{n} \cap F\right)$ and $\widetilde{x}^{F} \in$ $\operatorname{argmin} d_{2}\left(\bar{x},\left(\mathbb{Z}^{n} \cap F\right) \backslash \operatorname{Round}(\bar{x})\right)$.

(iii) It is indeed possible that $|\bar{X}|>1$ and the level sets are $\left(\alpha_{p}(\bar{x}, F), p\right)$-quasi-round w.r.t. all points in $\bar{X}$, compare the example in Figure 2.4: the set $\mathcal{L}_{\leq}\left(f\left(x^{*}\right)\right)$ is $\left(\alpha_{2}\left(\bar{x}, \mathbb{R}^{2}\right), 2\right)$ quasi-round w.r.t. $\bar{x}$ for all $\bar{x} \in \bar{X}$ (since it is $\left(\alpha_{2}, 2\right)$-quasi-round w.r.t. $\bar{x}$ for all $\bar{x} \in \bar{X}$, compare Corollary 2.12).

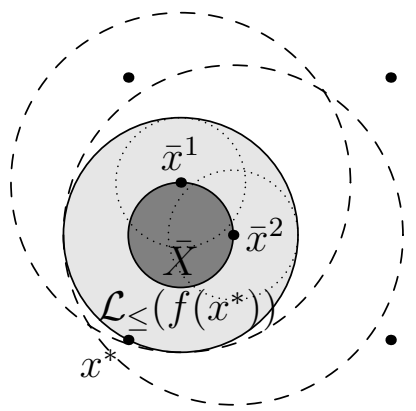

Figure 2.4.: The depicted level set is $\left(\alpha_{2}\left(\bar{x}, \mathbb{R}^{2}\right), 2\right)$-quasi-round w.r.t. $\bar{x}$ for all $\bar{x} \in \bar{X}$.

To apply Theorem 2.5 it is necessary to calculate $\alpha_{p}(\bar{x}, F)$ but as it depends on $F$ this cannot be done in general. Therefore it might be helpful to consider the following alternative formulation.

Theorem 2.8 (Rounding Property for quasi-round level sets). Let $(f, F)$ be given. Assume that for any optimal solution $\bar{x}$ to $(C P)$ and for any level $f(\bar{x}) \leq z \leq \min \{f(x): f \in$ $\operatorname{Round}(\bar{x}) \cap F\}$ there exists $p \in[1, \infty]$ such that the level set $\mathcal{L}_{\leq}^{F}(z)$ is $\left(\alpha_{p}(\bar{x}), p\right)$-quasi-round with respect to $\bar{x}$, where

$$
\alpha_{p}(\bar{x}):=d_{p}\left(\bar{x}, \mathbb{Z}^{n} \backslash \operatorname{Round}(\bar{x})\right)-d_{p}\left(\bar{x}, \mathbb{Z}^{n}\right) .
$$

Then $(f, F)$ has the Rounding Property.

Proof. Analogously to the proof of Theorem 2.5.

If $F=\mathbb{R}^{n}$ we have that $\alpha_{p}\left(\bar{x}, \mathbb{R}^{n}\right)=\alpha_{p}(\bar{x})$ but in general we cannot say whether $\alpha_{p}(\bar{x}, F)$ or $\alpha_{p}(\bar{x})$ is smaller, and therefore imposes a stricter assumption, compare the following example. 
Example 2.9. In Figure $2.5 d_{2}\left(\bar{x}, \mathbb{Z}^{n} \cap F\right)=\left\|\bar{x}-\lfloor\bar{x}\rceil^{F}\right\|_{2}>\|\bar{x}-\lfloor\bar{x}\rceil\|_{2}=d_{2}\left(\bar{x}, \mathbb{Z}^{n}\right)$ whereas $d_{2}\left(\bar{x},\left(\mathbb{Z}^{n} \cap F\right) \backslash \operatorname{Round}(\bar{x})\right)=d_{2}\left(\bar{x}, \mathbb{Z}^{n} \backslash \operatorname{Round}(\bar{x})\right)=\|\bar{x}-\widetilde{x}\|_{2}$ and therefore

$$
\begin{aligned}
\alpha_{2}(\bar{x}) & =d_{2}\left(\bar{x}, \mathbb{Z}^{n} \backslash \operatorname{Round}(\bar{x})\right)-d_{2}\left(\bar{x}, \mathbb{Z}^{n}\right) \\
& >d_{2}\left(\bar{x},\left(\mathbb{Z}^{n} \cap F\right) \backslash \operatorname{Round}(\bar{x})\right)-d_{2}\left(\bar{x}, \mathbb{Z}^{n} \cap F\right)=\alpha_{2}(\bar{x}, F) .
\end{aligned}
$$

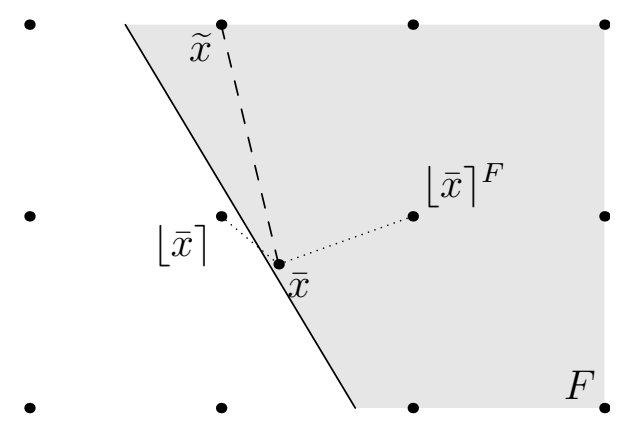

Figure 2.5.: Situation where $\alpha_{2}(\bar{x})>\alpha_{2}(\bar{x}, F)$.

On the other hand in Figure $2.6 d_{2}\left(\bar{x}, \mathbb{Z}^{n} \cap F\right)=d_{2}\left(\bar{x}, \mathbb{Z}^{n}\right)=\|\bar{x}-\lfloor\bar{x}\rceil\|_{2}$ and $d_{2}\left(\bar{x},\left(\mathbb{Z}^{n} \cap F\right) \backslash\right.$ $\operatorname{Round}(\bar{x}))=:\left\|\bar{x}-\widetilde{x}^{F}\right\|_{2}>\|\bar{x}-\widetilde{x}\|_{2}=d_{2}\left(\bar{x}, \mathbb{Z}^{n} \backslash \operatorname{Round}(\bar{x})\right)$ and therefore

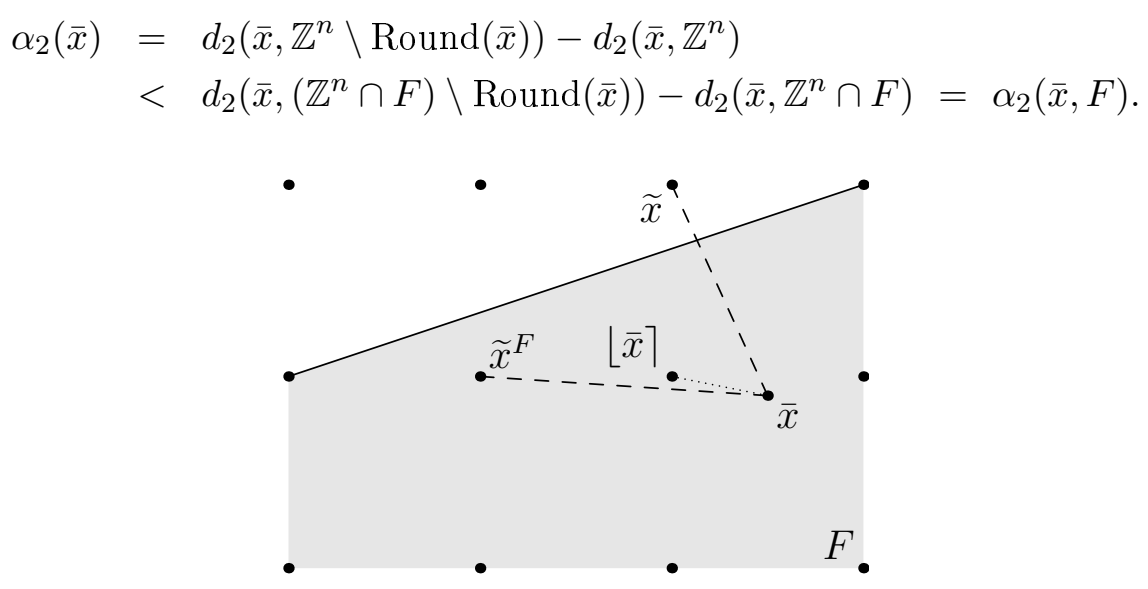

Figure 2.6.: Situation where $\alpha_{2}(\bar{x})<\alpha_{2}(\bar{x}, F)$.

It might seem odd that nevertheless both guarantee the Rounding Property. The reason is that in fact $\alpha_{p}^{\prime}(\bar{x}, F):=d_{p}\left(\bar{x},\left(\mathbb{Z}^{n} \cap F\right) \backslash \operatorname{Round}(\bar{x})\right)-d_{p}\left(\bar{x}, \mathbb{Z}^{n}\right)$ is sufficient for the Rounding Property (see Theorem 2.10) and

$$
\begin{aligned}
\alpha_{p}(\bar{x}) & =d_{p}\left(\bar{x}, \mathbb{Z}^{n} \backslash \operatorname{Round}(\bar{x})\right)-d_{p}\left(\bar{x}, \mathbb{Z}^{n}\right) \\
& \leq d_{p}\left(\bar{x},\left(\mathbb{Z}^{n} \cap F\right) \backslash \operatorname{Round}(\bar{x})\right)-d_{p}\left(\bar{x}, \mathbb{Z}^{n}\right)=\alpha_{p}^{\prime}(\bar{x}, F) . \\
\alpha_{p}(\bar{x}, F) & =d_{p}\left(\bar{x},\left(\mathbb{Z}^{n} \cap F\right) \backslash \operatorname{Round}(\bar{x})\right)-d_{p}\left(\bar{x}, \mathbb{Z}^{n} \cap F\right) \\
& \leq d_{p}\left(\bar{x},\left(\mathbb{Z}^{n} \cap F\right) \backslash \operatorname{Round}(\bar{x})\right)-d_{p}\left(\bar{x}, \mathbb{Z}^{n}\right)=\alpha_{p}^{\prime}(\bar{x}, F) .
\end{aligned}
$$


Theorem 2.10 (Rounding Property for quasi-round level sets). Let $(f, F)$ be given. Assume that for any optimal solution $\bar{x}$ to $(C P)$ and for any level $f(\bar{x}) \leq z \leq \min \{f(x): f \in$ $\operatorname{Round}(\bar{x}) \cap F\}$ there exists $p \in[1, \infty]$ such that the level set $\mathcal{L}_{\leq}^{F}(z)$ is $\left(\alpha_{p}^{\prime}(\bar{x}, F), p\right)$-quasiround with respect to $\bar{x}$, where

$$
\alpha_{p}^{\prime}(\bar{x}, F):=d_{p}\left(\bar{x},\left(\mathbb{Z}^{n} \cap F\right) \backslash \operatorname{Round}(\bar{x})\right)-d_{p}\left(\bar{x}, \mathbb{Z}^{n}\right) .
$$

Then $(f, F)$ has the Rounding Property.

Proof. The proof is analogous to the ones of Theorems 2.5 and 2.8 .

As mentioned before, to apply one of the Theorems $2.5,2.8$ or 2.10 it is crucial to know the respective $\alpha$. Since $\alpha_{p}(\bar{x}, F)$ and $\alpha_{p}^{\prime}(\bar{x}, F)$ depend on $F$ we are not able to compute them without knowing $F$ and we restrict our analysis to $\alpha_{p}(\bar{x})$.

Lemma 2.11. For a given $y \in \mathbb{R}^{n}$ we define $a_{j}:=\left|\left\lfloor y_{j}\right\rceil-y_{j}\right|$ and choose $i \in \operatorname{argmin}_{j=1, \ldots, n} a_{j}$. Then we get that $\alpha_{p}(y):=d_{p}\left(y, \mathbb{Z}^{n} \backslash \operatorname{Round}(y)\right)-d_{p}\left(y, \mathbb{Z}^{n}\right)$ is given by:

(i) for $p=\infty$ :

$$
\alpha_{\infty}(y)=1+a_{i}-\max _{j=1, \ldots, n} a_{j}
$$

(ii) for $p \in[1, \infty)$ :

$$
\alpha_{p}(y)=\sqrt[p]{\left(a_{i}+1\right)^{p}+\sum_{j \neq i} a_{j}^{p}}-\sqrt[p]{\sum_{j=1}^{n} a_{j}^{p}} .
$$

Proof. By Lemma 1.20 we have $d_{p}\left(y, \mathbb{Z}^{n}\right)=\|\lfloor y\rceil-y\|_{p}$ for $p \in[1, \infty]$.

For $\widetilde{y} \in \mathbb{Z}^{n}$ with

$$
\widetilde{y}_{j}:= \begin{cases}\left\lceil y_{i}\right\rceil+1 & \text { if } j=i \text { and }\left\lfloor y_{i}\right\rceil=\left\lceil y_{i}\right\rceil \\ \left\lfloor y_{i}\right\rfloor-1 & \text { if } j=i \text { and }\left\lfloor y_{i}\right\rceil=\left\lfloor y_{i}\right\rfloor \neq y_{i} \\ \left\lfloor y_{j}\right\rceil & \text { if } j \neq i\end{cases}
$$

we have $\widetilde{y} \in \underset{x \in \mathbb{Z}^{n} \backslash \operatorname{Round}(y)}{\operatorname{argmin}}\|x-y\|_{p}$, i.e., the closest, not rounded point w.r.t. $y$ :

- $\widetilde{y} \notin \operatorname{Round}(y)$ as for all $x \in \operatorname{Round}(y)$ and for all $j=1, \ldots, n$ we have $\left|x_{j}-y_{j}\right|<1$ but as $a_{i} \geq 0$ we get $\left|\widetilde{y}_{i}-y_{i}\right|=a_{i}+1 \geq 1$.

- $\|\widetilde{y}-y\|_{p} \leq\|x-y\|_{p}$ for all $x \in \mathbb{Z}^{n} \backslash \operatorname{Round}(y)$ :

As $x \notin \operatorname{Round}(y)$ there exists $k \in\{1, \ldots, n\}$ with $\left|x_{k}-y_{k}\right| \geq 1$ and hence $\left|x_{k}-y_{k}\right| \geq 1+a_{k}$ as $x_{k} \in \mathbb{Z}$. Furthermore, we have $\left|x_{j}-y_{j}\right| \geq a_{j}$ for all $j \in\{1, \ldots, n\}$ as $x_{j} \in \mathbb{Z}$.

(i) Let $p=\infty$ : As $\|x-y\|_{\infty} \geq 1+a_{k}$ and $\|\widetilde{y}-y\|_{\infty}=1+a_{i}$ we have $\|x-y\|_{\infty} \geq 1+$ $a_{k} \geq 1+a_{i}=\|\widetilde{y}-y\|_{\infty}$.

(ii) For $p \in[1, \infty)$ we get $\|\widetilde{y}-y\|_{p}^{p}-\|x-y\|_{p}^{p}=\sum_{j \neq i} a_{j}^{p}+\left(1+a_{i}\right)^{p}-\sum_{j=1}^{n}\left|x_{j}-y_{j}\right|^{p}$. If $k=i$ we have $\|\widetilde{y}-y\|_{p}^{p}-\|x-y\|_{p}^{p} \leq\left(1+a_{i}\right)^{p}-\left|x_{i}-y_{i}\right|^{p} \leq 0$.

Otherwise, if $k \neq i$ we have $\|\widetilde{y}-y\|_{p}^{p}-\|x-y\|_{p}^{p} \leq a_{k}^{p}+\left(1+a_{i}\right)^{p}-a_{i}^{p}-\left(1+a_{k}\right)^{p} \leq 0$ as $a_{k}^{p}-\left(1+a_{k}\right)^{p} \leq a_{i}^{p}-\left(1+a_{i}\right)^{p}$ because $x^{p}-(1+x)^{p}$ is monotonically decreasing for $p \geq 1$ and $a_{i} \leq a_{k}$. 
This means,

(i) for $p=\infty$ : $\alpha_{\infty}(y)=\|\widetilde{y}-y\|_{\infty}-\|\lfloor y\rceil-y\|_{\infty}=1+a_{i}-\max _{j=1, \ldots, n} a_{j}$

(ii) and for $p \in[1, \infty): \alpha_{p}(y)=\|\widetilde{y}-y\|_{p}-\|\lfloor y\rceil-y\|_{p}=\sqrt[p]{\left(a_{i}+1\right)^{p}+\sum_{j \neq i} a_{j}^{p}}-\sqrt[p]{\sum_{j=1}^{n} a_{j}^{p}}$.

We can generalize $\alpha_{p}(\bar{x})$ even further by making it independent of $\bar{x}$.

Corollary 2.12. Let $(f, F)$ be given. Assume that for any optimal solution $\bar{x}$ to $(C P)$ and for any level $f(\bar{x}) \leq z \leq \min \{f(x): f \in \operatorname{Round}(\bar{x}) \cap F\}$ there exists $p \in[1, \infty]$ such that the level set $\mathcal{L}_{\leq}^{F}(z)$ is $\left(\alpha_{p}, p\right)$-quasi-round with respect to $\bar{x}$, where

$$
\alpha_{p}:=\min _{y \in \mathbb{R}^{n}}\left\{d_{p}\left(y, \mathbb{Z}^{n} \backslash \operatorname{Round}(y)\right)-d_{p}\left(y, \mathbb{Z}^{n}\right)\right\} .
$$

Then $(f, F)$ has the Rounding Property.

Proof. Since $\alpha_{p} \leq \alpha_{p}(\bar{x})$ the result follows from Theorem 2.8 and Remark 2.3 (iv).

We can calculate $\alpha_{p}$ explicitly, depending on $p$ and $n$.

Lemma 2.13. (i) For $p=\infty$ we have

$$
\alpha_{\infty}:=\min _{y \in \mathbb{R}^{n}}\left\{d_{\infty}\left(y, \mathbb{Z}^{n} \backslash \operatorname{Round}(y)\right)-d_{\infty}\left(y, \mathbb{Z}^{n}\right)\right\}=0.5
$$

(ii) For $p \in[1, \infty)$ we have

$$
\alpha_{p}:=\min _{y \in \mathbb{R}^{n}}\left\{d_{p}\left(y, \mathbb{Z}^{n} \backslash \operatorname{Round}(y)\right)-d_{p}\left(y, \mathbb{Z}^{n}\right)\right\}=0.5 \cdot\left(\sqrt[p]{n-1+2^{p}}-\sqrt[p]{n-1}\right) .
$$

Proof. (i) Let $p=\infty$ : using Lemma 2.11 we have to solve

$$
\alpha_{\infty}=\min _{y \in \mathbb{R}^{n}}\left\{1+\min _{j=1, \ldots, n} a_{j}-\max _{j=1, \ldots, n} a_{j}\right\}
$$

where $a_{j}:=\left|\left\lfloor y_{j}\right\rceil-y_{j}\right|$.

As $0 \leq a_{j} \leq 0.5$ for all $j=1, \ldots, n$ we get $\alpha_{\infty} \geq 0.5$. On the other hand for $y=$ $(0.5,1, \ldots, 1) \in \mathbb{R}^{n}$ we get: $\alpha_{\infty}(y)=0.5$ and therefore $\alpha_{\infty} \leq 0.5$.

(ii) Let $p \in[1, \infty)$ : using Lemma 2.11 we have to solve

$$
\alpha_{p}=\min _{y \in \mathbb{R}^{n}} \sqrt[p]{\left(a_{i}+1\right)^{p}+\sum_{j \neq i} a_{j}^{p}}-\sqrt[p]{\sum_{j=1}^{n} a_{j}^{p}}
$$


where $a_{j}:=\left|\left\lfloor y_{j}\right\rceil-y_{j}\right|$ and $i \in \operatorname{argmin}_{j=1, \ldots, n} a_{j}$, fixed, i.e.,

$$
\begin{aligned}
\alpha_{p}=\min & \sqrt[p]{\left(1+a_{i}\right)^{p}+\sum_{j \neq i} a_{j}^{p}}-\sqrt[p]{\sum_{j=1}^{n} a_{j}^{p}} \\
\text { s.t. } & 0 \leq a_{i} \leq a_{j} \leq 0.5 \quad \forall j=1, \ldots, n
\end{aligned}
$$

For $p=1$ we see directly that $\alpha_{1}=1$.

For $p>1$ we consider first the problem $\min \{\sqrt[p]{b+x}-\sqrt[p]{c+x}: l \leq x \leq u\}$, with $l \geq 0$ and $b>c \geq 0$. The objective function decreases with $x \geq 0$ as

$$
\frac{d}{d x}(\sqrt[p]{b+x}-\sqrt[p]{c+x})=\frac{1}{p}\left((\underbrace{b+x}_{\geq c+x})^{1 / p-1}-(c+x)^{1 / p-1}\right) \leq 0
$$

(since $\frac{1}{p}-1<0$ ) and hence the minimum is attained when $x=u$. So in our problem the optimal $a$ satisfies $a_{j}=\frac{1}{2}$ for $j \neq i$ and we are left with $\min \left\{\sqrt[p]{\left(1+a_{i}\right)^{p}+d}-\right.$ $\left.\sqrt[p]{a_{i}^{p}+d}: 0 \leq a_{i} \leq \frac{1}{2}\right\}$, where $d=(n-1) / 2^{p}$. The above function of $a_{i}$ is differentiable and its derivative is $\left(\left(1+a_{i}\right)^{p}+d\right)^{\frac{1}{p}-1}\left(1+a_{i}\right)^{p-1}-\left(a_{i}^{p}+d\right)^{\frac{1}{p}-1} a_{i}^{p-1}$, which is obviously nonnegative when $a_{i} \geq 0$. So the function is monotonically increasing in $a_{i}$ and the minimum is attained when $a_{i}=0$, hence the result.

We show in an easy example that the different $\alpha$ s we introduced might indeed all be different.

Example 2.14. Let $n=p=2, \bar{x}=(0.8,0.6)$ and $F=\left\{(x, y) \in \mathbb{R}^{2}: y \leq-x+1.6\right\}$, compare Figure 2.\%.

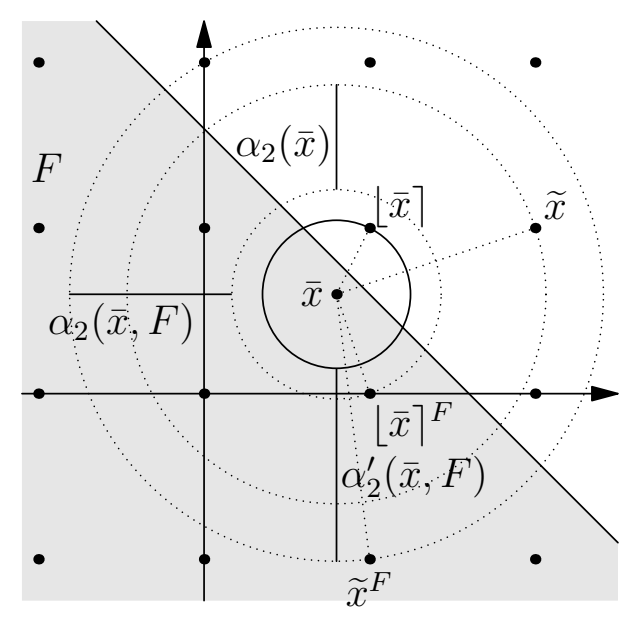

Figure 2.7.: Comparing the different $\alpha$ s used in this section. 
Then we get $\lfloor\bar{x}\rceil=(1,1),\lfloor\bar{x}\rceil^{F}=(1,0), \widetilde{x}=(2,1)$ and $\widetilde{x}^{F}=(1,-1)$ and therefore

$$
\begin{aligned}
\alpha_{2}^{\prime}(\bar{x}, F) & :=\left\|\widetilde{x}^{F}-\bar{x}\right\|_{2}-\|\lfloor\bar{x}\rceil-\bar{x}\|_{2} \approx 1.165 \\
\alpha_{2}(\bar{x}, F) & :=\left\|\widetilde{x}^{F}-\bar{x}\right\|_{2}-\left\|\lfloor\bar{x}\rceil^{F}-\bar{x}\right\|_{2} \approx 0.98 \\
\alpha_{2}(\bar{x}) & :=\|\widetilde{x}-\bar{x}\|_{2}-\|\lfloor\bar{x}\rceil-\bar{x}\|_{2} \approx 0.818 \\
\alpha_{2} & :=\min _{x \in \mathbb{R}^{2}}\left(d_{2}\left(x, \mathbb{Z}^{2} \backslash \operatorname{Round}(x)\right)-d_{2}\left(x, \mathbb{Z}^{2}\right)\right)=\frac{1}{2}(\sqrt{5}-1) \approx 0.618 .
\end{aligned}
$$

This means of course that there might be problems where we are able to detect the Rounding Property by using Theorem 2.10 but not by using Theorem 2.5, Theorem 2.8 or Corollary 2.12, compare the example in Figure 2.8.

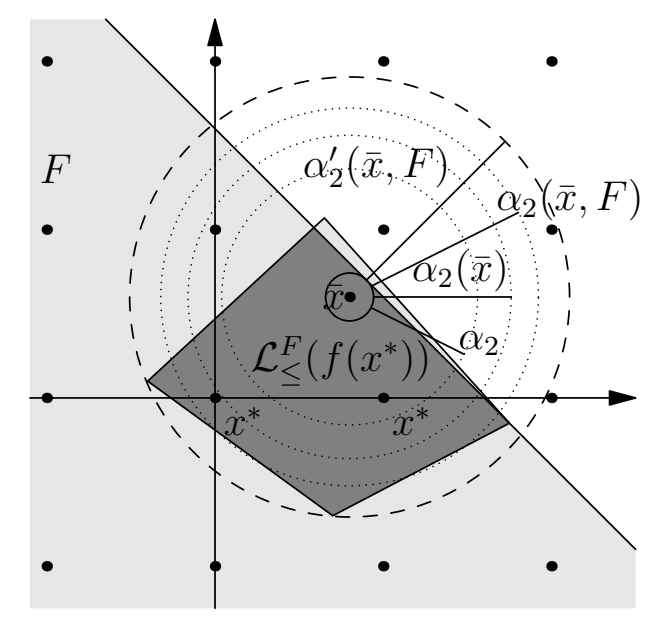

Figure 2.8.: $(f, F)$ has the Rounding Property, but is only $\left(\alpha_{2}^{\prime}(\bar{x}, F), 2\right)$-quasi-round and not $\left(\alpha_{2}(\bar{x}, F), 2\right)-,\left(\alpha_{2}(\bar{x}), 2\right)$ - or $\left(\alpha_{2}, 2\right)$-quasi-round.

In the following we collect some properties of $\alpha_{p}$. To make clear that it depends on $n$ we denote $\alpha_{p}(n)=0.5\left(\sqrt[p]{n-1+2^{p}}-\sqrt[p]{n-1}\right)$.

We start by fixing $p$ and investigating how $\alpha_{p}(n)$ depends on $n$. Let $p \in[1, \infty]$ be fixed:

(i) $p=\infty: \alpha_{\infty}(n)=0.5$ for all $n \geq 1$

(ii) $p=1: \alpha_{1}(n)=1$ for all $n \geq 1$

(iii) $p \in(1, \infty): \alpha_{p}(1)=1$ and $\alpha_{p}$ is monotonically decreasing in $n$ :

$$
\begin{gathered}
\frac{d}{d n} \alpha_{p}=\frac{1}{2 p} \cdot\left[\frac{1}{\left(n-1+2^{p}\right)^{1-1 / p}}-\frac{1}{(n-1)^{1-1 / p}}\right]<0 . \\
\Rightarrow \quad 0 \leq \alpha_{p}(n) \leq 1 \text { for all } p \in[1, \infty], \text { for all } n \geq 1 .
\end{gathered}
$$

(iv) $\lim _{n \rightarrow \infty} \alpha_{p}(n)=0$ for all fixed $p \in(1, \infty)$.

Compare Figure 2.9 for graphs of $\alpha_{p}(n)$ for some fixed values of $p$.

A smaller value of $\alpha$ means that the set has to be "rounder". This means that for higher dimensions $n$ the level sets have to be rounder to guarantee the Rounding Property. This effect occurs later for higher values of $p$. 


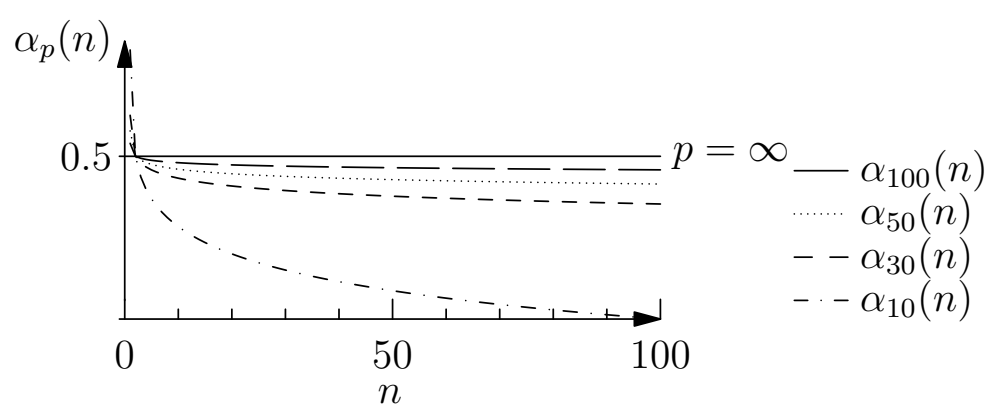

Figure 2.9.: Graphs of $\alpha_{p}(n)$ for some fixed values of $p$.

Now we fix $n$ and see how $\alpha_{p}(n)$ depends on $p$. Let $n \geq 1$ be fixed:

(i) $n=1: \alpha_{p}(1)=1$ for $p \in[1, \infty)$

(ii) $\lim _{p \rightarrow \infty} \alpha_{p}(n)=\alpha_{\infty}=0.5$ for all $n>1$ :

$$
\lim _{p \rightarrow \infty} \alpha_{p}(n)=\lim _{p \rightarrow \infty} 0.5[\underbrace{\sqrt[p]{n-1+2^{p}}}_{\rightarrow 2}-\underbrace{\sqrt[p]{n-1}}_{\rightarrow 1}]=0.5 .
$$

Compare Figure 2.10 for graphs of $\alpha_{p}(n)$ for some fixed values of $n$.

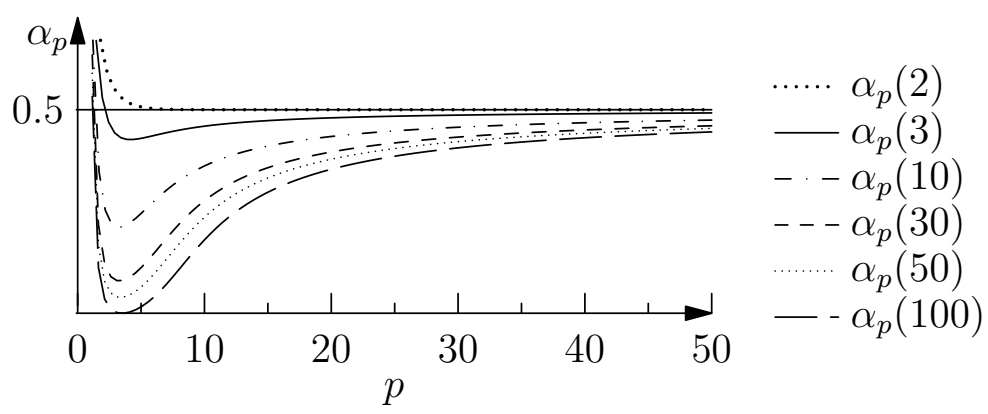

Figure 2.10.: Graphs of $\alpha_{p}(n)$ for some fixed values of $n$.

We observe an eye-catching minimum for each dimension $n \geq 3$ around $p=3$. Even though it might look like this, the minima are not all at the same position but differ for different values of $n$. This might suggest that it is more unlikely that a set is $\left(\alpha_{3}, 3\right)$-quasi-round than for example $\left(\alpha_{20}, 20\right)$-quasi-round. Note however that whether or not a set is $\left(\alpha_{p}, p\right)$-quasi-round does not only depend on the value of $\alpha_{p}$, but also on the question whether its shape is similar to the $p$-norm unit ball.

At this point we explain why we restrict $R-r$ and not for example $\frac{r}{R}$ in the definition of quasi-roundness (compare Definition 2.1). We want to make sure that as soon as $R \geq$ $\min _{x \in \mathbb{Z}^{n} \backslash \operatorname{Round}(\bar{x})}\|x-\bar{x}\|_{p}$ we have that $r \geq \min _{x \in \mathbb{Z}^{n}}\|x-\bar{x}\|_{p}$ (ignoring $F$ at this point for the sake of readability). We could also restrict $\frac{r}{R}$ (as for example in the closely related definition 
of the asphericity of a set (compare Dvoretzky [1963]) to enforce this property. This would mean to require

$$
\frac{r}{R} \geq \frac{\min _{x \in \mathbb{Z}^{n}}\|x-\bar{x}\|_{p}}{\min _{x \in \mathbb{Z}^{n} \backslash \operatorname{Round}(\bar{x})}\|x-\bar{x}\|_{p}} .
$$

On the other hand in Theorem 2.8 we restrict

$$
r \geq R-\min _{x \in \mathbb{Z}^{n} \backslash \operatorname{Round}(\bar{x})}\|x-\bar{x}\|_{p}+\min _{x \in \mathbb{Z}^{n}}\|x-\bar{x}\|_{p}
$$

This means that for $R \leq \min _{x \in \mathbb{Z}^{n} \backslash \operatorname{Round}(\bar{x})}\|x-\bar{x}\|_{p}$ we have that (2.1) gives a stricter bound than (2.2):

$$
\begin{aligned}
& R \leq \min _{x \in \mathbb{Z}^{n} \backslash \operatorname{Round}(\bar{x})}\|x-\bar{x}\|_{p} \\
& \Leftrightarrow \quad R \cdot(\underbrace{\min _{x \in \mathbb{Z}^{n} \backslash \operatorname{Round}(\bar{x})}\|x-\bar{x}\|_{p}-\min _{x \in \mathbb{Z}^{n}}\|x-\bar{x}\|_{p}}_{>0}) \leq\left(\min _{x \in \mathbb{Z}^{n} \backslash \operatorname{Round}(\bar{x})}\|x-\bar{x}\|_{p}\right)^{2} \\
& -\min _{x \in \mathbb{Z}^{n} \backslash \operatorname{Round}(\bar{x})}\|x-\bar{x}\|_{p} \cdot \min _{x \in \mathbb{Z}^{n}}\|x-\bar{x}\|_{p} \\
& \Leftrightarrow \quad R-\min _{x \in \mathbb{Z}^{n} \backslash \operatorname{Round}(\bar{x})}\|x-\bar{x}\|_{p}+\min _{x \in \mathbb{Z}^{n}}\|x-\bar{x}\|_{p} \leq R \cdot \frac{\min _{x \in \mathbb{Z}^{n}}\|x-\bar{x}\|_{p}}{\min _{x \in \mathbb{Z}^{n} \backslash \operatorname{Round}(\bar{x})}\|x-\bar{x}\|_{p}}
\end{aligned}
$$

As soon as $R>\min _{x \in \mathbb{Z}^{n} \backslash \operatorname{Round}(\bar{x})}\|x-\bar{x}\|_{p}$ we have $r \geq \min _{x \in \mathbb{Z}^{n}}\|x-\bar{x}\|_{p}$ in both (2.1) and (2.2) and hence there is at least one rounded point in the corresponding level set and hence we are not interested in this case. This means that for all cases we are interested in (2.1) gives a stricter bound and hence we prefer to restrict $R-r$ instead of $\frac{r}{R}$.

Next we investigate which function manipulations keep the quasi-roundness of the level sets and therefore induce functions that have the Rounding Property.

Lemma 2.15. (i) Let $f: \mathbb{R}^{n} \rightarrow \mathbb{R}$ and assume that for any optimal solution $\bar{x}$ to $(C P)$ and for any level $f(\bar{x}) \leq z \leq \min \{f(x): x \in \operatorname{Round}(\bar{x})\}$ there exists $p \in[1, \infty]$ such that the level set $\mathcal{L}_{\leq, f}(z)$ is $\left(\alpha_{p}(\bar{x}), p\right)$-quasi-round w.r.t. $\bar{x}$.

a) Define $g_{1}(x):=f(x)+\gamma$ where $\gamma \in \mathbb{R}$.

b) Define $g_{2}(x):=\gamma f(x)$ where $\gamma \in \mathbb{R}, \gamma \geq 0$.

(ii) Let $f: \mathbb{R}^{n} \rightarrow \mathbb{R}, a \in \mathbb{R}^{n}$. Define $g_{3}(x):=f(x-a)$. A ssume that for any optimal solution $\bar{x}$ to $(C P)$ and for any level $g_{3}(\bar{x}) \leq z \leq \min \left\{g_{3}(x): x \in \operatorname{Round}(\bar{x}+a)\right\}$ there exists $p \in[1, \infty]$ such that the level set $\mathcal{L}_{\leq, f}(z)$ is $\left(\alpha_{p}(\bar{x}+a), p\right)$-quasi-round w.r.t. $\bar{x}$.

(iii) Let $f_{1}: \mathbb{R}^{n} \rightarrow \mathbb{R}, f_{2}: \mathbb{R}^{n} \rightarrow \mathbb{R}$ and assume that the sets of continuous minimizers coincide: $\bar{X}_{1}=\bar{X}_{2}$.

a) Assume that there exists $p \in[1, \infty]$ such that for $i \in\{1,2\}$ : for any optimal solution $\bar{x} \in \bar{X}_{i}$ and for any level $f_{i}(\bar{x}) \leq z \leq \min \left\{f_{i}(x): x \in \operatorname{Round}(\bar{x})\right\}$ the level set $\mathcal{L}_{\leq, f_{i}}(z)$ is $\left(\alpha_{p}(\bar{x}), p\right)$-quasi-round w.r.t. $\bar{x}$. Define $g_{4}(x):=\min \left\{f_{1}(x), f_{2}(x)\right\}$. 
b) Assume that there exists $p \in[1, \infty]$ such that for $i \in\{1,2\}$ : for any optimal solution $\bar{x} \in \bar{X}_{i}$ and for any level $f_{i}(\bar{x}) \leq z \leq \max \left\{\min \left\{f_{1}(x): x \in \operatorname{Round}(\bar{x})\right\}, \min \left\{f_{2}(x):\right.\right.$ $x \in \operatorname{Round}(\bar{x})\}\}$ the level set $\mathcal{L}_{\leq, f_{i}}(z)$ is $\left(\alpha_{p}(\bar{x}), p\right)$-quasi-round w.r.t. $\bar{x}$. Define $g_{5}(x):=\max \left\{f_{1}(x), f_{2}(x)\right\}$.

Then for each $i \in\{1, \ldots, 5\}$ there exists $p \in[1, \infty]$ for any optimal solution $\bar{x} \in \min \left\{g_{i}(x)\right.$ : $\left.x \in \mathbb{R}^{n}\right\}$ and for any level $g_{i}(\bar{x}) \leq z \leq \min \left\{g_{i}(x): x \in \operatorname{Round}(\bar{x})\right\}$ such that $\mathcal{L}_{\leq, g_{i}}(z)$ is $\left(\alpha_{p}(\bar{x}), p\right)$-quasi-round w.r.t. $\bar{x}$ and hence $\left(g_{i}, \mathbb{R}^{n}\right)$ has the Rounding Property with Theorem 2.8.

Proof. $\quad g_{1}$ : Let $\bar{x} \in \operatorname{argmin}\left\{g_{1}(x): x \in \mathbb{R}^{n}\right\}$. Then we have also $\bar{x} \in \bar{X}$ and hence for all $f(\bar{x}) \leq z \leq \min \{f(x): x \in \operatorname{Round}(\bar{x})\}$ there exists $p \in[1, \infty]$ such that $\mathcal{L}_{\leq, f}(z)$ is $\left(\alpha_{p}(\bar{x}), p\right)$-quasi-round w.r.t. $\bar{x}$. Since $\mathcal{L}_{\leq, g_{1}}(z)=\mathcal{L}_{\leq, f}(z-\gamma)$, this means that for all $g_{1}(\bar{x}) \leq z \leq \min \left\{g_{1}(x): x \in \operatorname{Round}(\bar{x})\right\}$ there exists $p \in[1, \infty]$ such that $\mathcal{L}_{\leq, g_{1}}(z)$ is $\left(\alpha_{p}(\bar{x}), p\right)$-quasi-round w.r.t. $\bar{x}$.

$g_{2}$ : The result for $g_{2}$ follows analogously if $\gamma>0$ with the observation that $\mathcal{L}_{\leq, g_{2}}(z)=$ $\mathcal{L}_{\leq, f}\left(\frac{z}{\gamma}\right)$. For $\gamma=0$ we have $g_{2}(x) \equiv 0$ and hence $\bar{X}=\mathbb{R}^{n}$ and $X^{*}=\mathbb{Z}^{n}$.

$g_{3}$ : Let $\bar{x} \in \operatorname{argmin}\left\{g_{3}(x): x \in \mathbb{R}^{n}\right\}$. Then we have $\bar{x}-a \in \bar{X}$ and hence for all levels $g_{3}(\bar{x}) \leq z \leq \min \left\{g_{3}(x): x \in \operatorname{Round}(\bar{x})\right\}$ there exists $p \in[1, \infty]$ such that the level set $\mathcal{L}_{\leq, f}(z)$ is $\left(\alpha_{p}(\bar{x}), p\right)$-quasi-round w.r.t. $\bar{x}-a$. Furthermore, $\mathcal{L}_{\leq, g_{3}}(z)=\mathcal{L}_{\leq, f}(z)+a$ and hence $\mathcal{L}_{\leq, g_{3}}(z)$ is $\left(\alpha_{p}(\bar{x}), p\right)$-quasi-round w.r.t. $\bar{x}$ due to Lemma 2.4.

$g_{4}$ : Let $\bar{x} \in \operatorname{argmin}\left\{g_{4}(x): x \in \mathbb{R}^{n}\right\}$. Then we have $\bar{x} \in \bar{X}_{1}=\bar{X}_{2}$ and hence there exists $p \in[1, \infty]$ such that for all $f_{1}(\bar{x}) \leq z \leq \min \left\{f_{1}(x): x \in \operatorname{Round}(\bar{x})\right\}$ the level set $\mathcal{L}_{\leq, f_{1}}(z)$ is $\left(\alpha_{p}(\bar{x}), p\right)$-quasi-round w.r.t. $\bar{x}$ and for all $f_{2}(\bar{x}) \leq z \leq \min \left\{f_{2}(x): x \in \operatorname{Round}(\bar{x})\right\}$ the level set $\mathcal{L}_{\leq, f_{2}}(z)$ is $\left(\alpha_{p}(\bar{x}), p\right)$-quasi-round w.r.t. $\bar{x}$. This means that for all $g_{4}(\bar{x}) \leq$ $z \leq \min \left\{g_{4}(x): x \in \operatorname{Round}(\bar{x})\right\}=\min \left\{\min \left\{f_{1}(x): x \in \operatorname{Round}(\bar{x})\right\}, \min \left\{f_{2}(x): x \in\right.\right.$ $\operatorname{Round}(\bar{x})\}\}$ the level set $\mathcal{L}_{\leq, g_{4}}(z)=\mathcal{L}_{\leq, f_{1}}(z) \cup \mathcal{L}_{\leq, f_{2}}(z)$ is $\left(\alpha_{p}(\bar{x}), p\right)$-quasi-round w.r.t. $\bar{x}$, due to Lemma 2.4 .

$g_{5}:$ Let $\bar{x} \in \operatorname{argmin}\left\{g_{5}(x): x \in \mathbb{R}^{n}\right\}$. Then we have $\bar{x} \in \bar{X}_{1}=\bar{X}_{2}$ and hence there exists $p \in$ $[1, \infty]$ such that for all $f_{1}(\bar{x}) \leq z \leq \max \left\{\min \left\{f_{1}(x): x \in \operatorname{Round}(\bar{x})\right\}, \min \left\{f_{2}(x): x \in\right.\right.$ $\operatorname{Round}(\bar{x})\}\}$ the level set $\mathcal{L}_{\leq, f_{1}}(z)$ is $\left(\alpha_{p}(\bar{x}), p\right)$-quasi-round w.r.t. $\bar{x}$ and for all $f_{2}(\bar{x}) \leq$ $z \leq \max \left\{\min \left\{f_{1}(x): x \in \operatorname{Round}(\bar{x})\right\}, \min \left\{f_{2}(x): x \in \operatorname{Round}(\bar{x})\right\}\right\}$ the level set $\mathcal{L}_{\leq, f_{2}}(z)$ is $\left(\alpha_{p}(\bar{x}), p\right)$-quasi-round w.r.t. $\bar{x}$. This means that for all $g_{5}(\bar{x}) \leq z \leq$ $\min \left\{g_{5}(x): x \in \operatorname{Round}(\bar{x})\right\}=\max \left\{\min \left\{f_{1}(x): x \in \operatorname{Round}(\bar{x})\right\}, \min \left\{f_{2}(x): x \in\right.\right.$ $\operatorname{Round}(\bar{x})\}\}$ the level set $\mathcal{L}_{\leq, g_{5}}(z)=\mathcal{L}_{\leq, f_{1}}(z) \cap \mathcal{L}_{\leq, f_{2}}(z)$ is $\left(\alpha_{p}(\bar{x}), p\right)$-quasi-round w.r.t. $\bar{x}$, due to Lemma 2.4 .

The next question is, whether we even get the Strong Rounding Property as in Theorem 1.23. In general $\left(\alpha_{p}, p\right)$-quasi-roundness does not imply the Strong Rounding Property, compare Figure 2.11 for a counterexample. The point $\bar{x}$ is the unique continuous minimizer and the depicted level set $\mathcal{L}_{\leq}\left(f\left(x^{*}\right)\right)$ is $\left(\alpha_{2}, 2\right)$-quasi-round. Nevertheless, the problem does not have the Strong Rounding Property, as $\lfloor\bar{x}\rceil \notin X^{*}$. 


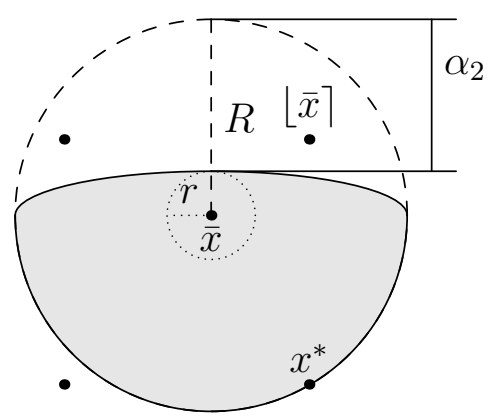

Figure 2.11.: $\left(\alpha_{p}, p\right)$-quasi-roundness is in general not enough to guarantee the Strong Rounding Property.

As we know on the other hand, that for $\alpha=0$, i.e., for round level sets we have the Strong Rounding Property (compare Theorem 1.23), it seems promising to decrease $\alpha$ to get the Strong Rounding Property. This is actually possible as the next theorem shows.

Theorem 2.16 (Strong Rounding Property for quasi-round level sets). Let $(f, F)$ be given. Assume that for any optimal solution $\bar{x}$ to $(C P)$ we have $\lfloor\bar{x}\rceil \in F$ and for any level $f(\bar{x}) \leq z \leq f(\lfloor\bar{x}\rceil)$ there exists a $p \in[1, \infty]$ such that the level set $\mathcal{L}_{\leq}^{F}(z)$ is $\left(\beta_{p}^{\prime}(\bar{x}, F), p\right)$ quasi-round with respect to $\bar{x}$, where

$$
\beta_{p}^{\prime}(\bar{x}, F):=d_{p}\left(\bar{x},\left(\mathbb{Z}^{n} \cap F\right) \backslash\{\lfloor\bar{x}\rceil\}\right)-d_{p}\left(\bar{x}, \mathbb{Z}^{n}\right) .
$$

Then $(f, F)$ has the Strong Rounding Property.

Proof. Let $\bar{x} \in \bar{X}$ and $x \in \mathbb{Z}^{n} \cap F$. Using Lemma 1.18 we show that $\lfloor\bar{x}\rceil \in \mathcal{L}_{\leq}^{F}(f(x))$. If $f(\lfloor\bar{x}\rceil) \leq f(x)$ we are done. Otherwise we have $x \neq\lfloor\bar{x}\rceil$ and there exists $p \in[1, \infty]$ such that $\mathcal{L}_{\leq}^{F}(f(x))$ is $\left(\beta_{p}^{\prime}(\bar{x}, F), p\right)$-quasi-round w.r.t. $\bar{x}$. This means that there exist $r, R \in \mathbb{R}_{0}^{+}$ such that $B_{p}(\bar{x}, r) \subseteq \mathcal{L}_{<}^{F}(f(x)) \subseteq B_{p}(\bar{x}, R)$ and $R-r \leq \beta_{p}^{\prime}(\bar{x}, F)$.

As $d_{p}\left(\bar{x}, \mathbb{Z}^{n}\right)=\|\bar{x}-\lfloor\bar{x}\rceil\|_{p}$, compare Lemma 1.20, we have

$$
\begin{aligned}
\|\bar{x}-\lfloor\bar{x}\rceil\|_{p} & =d_{p}\left(\bar{x},\left(\mathbb{Z}^{n} \cap F\right) \backslash\lfloor\bar{x}\rceil\right)-\beta_{p}^{\prime}(\bar{x}, F) \\
& \leq\|\bar{x}-x\|_{p}-\beta_{p}^{\prime}(\bar{x}, F) \quad \text { as } x \in \mathbb{Z}^{n} \cap F \text { and } x \neq\lfloor\bar{x}\rceil \\
& \leq R-\beta_{p}^{\prime}(\bar{x}, F) \quad \text { as } x \in \mathcal{L}_{\leq}^{F}(f(x)) \subseteq B_{p}(\bar{x}, R) \\
& \leq r .
\end{aligned}
$$

This means, $\lfloor\bar{x}\rceil \in B_{p}(\bar{x}, r) \subseteq \mathcal{L}_{\leq}^{F}(f(x))$ and hence $f(x)<f(\lfloor\bar{x}\rceil) \leq f(x)$. Like this $f(x)<$ $f(\lfloor\bar{x}\rceil)$ leads to a contradiction.

Note that we assume explicitly here that $\lfloor\bar{x}\rceil \in F$. This was not necessary in the corresponding theorem for the Rounding Property. The difference is that we assumed for example in Theorem 2.10 that the level sets are quasi-round for all $z \leq \min \{f(x): x \in \operatorname{Round}(\bar{x}) \cap F\}$. We state Theorem 2.16 in the way it is, since the advantage of the Strong Rounding Property is that we do not have to compare all points in $\operatorname{Round}(\bar{x}) \cap F$. Hence also the assumptions should not require knowledge about all those points. Furthermore, if $\lfloor\bar{x}\rceil \notin F(f, F)$ cannot 
have the Strong Rounding Property and hence it is good anyway to check this assumption first.

As for the Rounding Property we get the following two weaker formulations.

Corollary 2.17 (Strong Rounding Property for quasi-round level sets). Let $(f, F)$ be given. Assume that for any optimal solution $\bar{x}$ to $(C P)$ we have $\lfloor\bar{x}\rceil \in F$ and for any level $f(\bar{x}) \leq z \leq f(\lfloor\bar{x}\rceil)$ there exists $p \in[1, \infty]$ such that the level set $\mathcal{L}_{\leq}^{F}(z)$ is $\left(\beta_{p}(\bar{x}, F), p\right)$ - or $\left(\beta_{p}(\bar{x}), p\right)$-quasi-round with respect to $\bar{x}$, where

$$
\begin{gathered}
\beta_{p}(\bar{x}, F):=d_{p}\left(\bar{x},\left(\mathbb{Z}^{n} \cap F\right) \backslash\{\lfloor\bar{x}\rceil\}\right)-d_{p}\left(\bar{x}, \mathbb{Z}^{n} \cap F\right), \\
\beta_{p}(\bar{x}):=d_{p}\left(\bar{x}, \mathbb{Z}^{n} \backslash\{\lfloor\bar{x}\rceil\}\right)-d_{p}\left(\bar{x}, \mathbb{Z}^{n}\right) .
\end{gathered}
$$

Then $(f, F)$ has the Strong Rounding Property.

Proof. These are direct consequences of Theorem 2.16 since $\beta_{p}(\bar{x}, F) \leq \beta_{p}^{\prime}(\bar{x}, F)$ and $\beta_{p}(\bar{x}) \leq$ $\beta_{p}^{\prime}(\bar{x})$.

Notation 2.18. For the rest of this chapter we denote by $\hat{x}$ an element of $\operatorname{argmin} d_{p}\left(\bar{x}, \mathbb{Z}^{n} \backslash\right.$ $\{\lfloor\bar{x}\rceil\})$ and by $\hat{x}^{F}$ an element of $\operatorname{argmin} d_{p}\left(\bar{x},\left(\mathbb{Z}^{n} \cap F\right) \backslash\{\lfloor\bar{x}\rceil\}\right)$.

Just as in the case of $\alpha_{p}(\bar{x})$ and $\alpha_{p}(\bar{x}, F), \beta_{p}(\bar{x})=\beta_{p}(\bar{x}, F)$ if $F=\mathbb{R}^{n}$, but there is no general order between $\beta_{p}(\bar{x})$ and $\beta_{p}(\bar{x}, F)$ if $F \neq \mathbb{R}^{n}$, as can be seen for example in Figure 2.12.

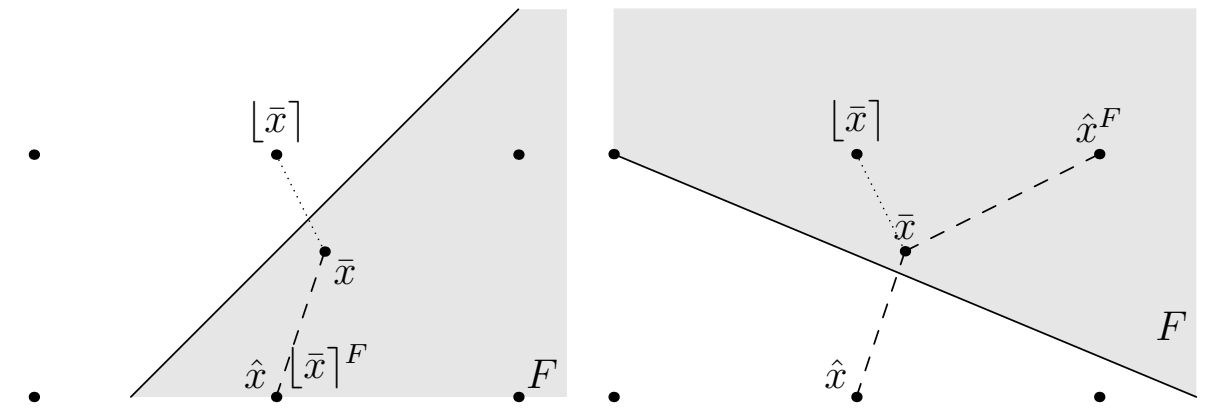

Figure 2.12.: In the left example we have $\beta_{p}(\bar{x})=\|\bar{x}-\hat{x}\|_{p}-\|\bar{x}-\lfloor\bar{x}\rceil\|_{p}>0=\beta_{p}(\bar{x}, F)$ whereas in the right picture we have $\beta_{p}(\bar{x})=\|\hat{x}-\bar{x}\|_{p}-\|\lfloor\bar{x}\rceil-\bar{x}\|_{p}<\| \hat{x}^{F}-$ $\bar{x}\left\|_{p}-\right\|\lfloor\bar{x}\rceil-\bar{x} \|_{p}=\beta_{p}(\bar{x}, F)$.

Now we investigate the value of $\beta_{p}(y)$.

Lemma 2.19. For a given $y \in \mathbb{R}^{n}$ define $a_{j}:=\left|\left\lfloor y_{j}\right\rceil-y_{j}\right|$ and choose $l \in \operatorname{argmax}_{j=1, \ldots, n} a_{j}$. Then $\beta_{p}(y):=d_{p}\left(y, \mathbb{Z}^{n} \backslash\{\lfloor\bar{x}\rceil\}\right)-d_{p}\left(y, \mathbb{Z}^{n}\right)$ is given by:

(i) for $p=\infty: \beta_{\infty}(y)=1-2 a_{l}$

(ii) for $p \in[1, \infty): \beta_{p}(y)=\sqrt[p]{\left(1-a_{l}\right)^{p}+\sum_{j \neq l} a_{j}^{p}}-\sqrt[p]{\sum_{j=1}^{n} a_{j}^{p}}$. 
Proof. By Lemma 1.20 we have $d_{p}\left(y, \mathbb{Z}^{n}\right)=\|\lfloor y\rceil-y\|_{p}$ for $p \in[1, \infty]$.

We define $\hat{y} \in \mathbb{Z}^{n}$ by

$$
\hat{y}_{j}:= \begin{cases}\left\lceil y_{l}\right\rceil & \text { if } j=l \text { and }\left\lfloor y_{l}\right\rceil=\left\lfloor y_{l}\right\rfloor \neq y_{l} \\ \left\lfloor y_{l}\right\rfloor & \text { if } j=l \text { and }\left\lfloor y_{l}\right\rceil=\left\lceil y_{l}\right\rceil \neq y_{l} \\ y_{l}+1 & \text { if } j=l \text { and } y_{l} \in \mathbb{Z} \\ \left\lfloor y_{j}\right\rceil & \text { if } j \neq l\end{cases}
$$

This means, $\left|y_{j}-\hat{y}_{j}\right|=a_{j}$ for all $j \neq l$ and $\left|y_{l}-\hat{y}_{l}\right|=1-a_{l}$. We show $\hat{y} \in \underset{x \in \mathbb{Z}^{n} \backslash\{\lfloor y\rceil\}}{\operatorname{argmin}}\|x-y\|_{p}$ :

- Obviously $\hat{y} \neq\lfloor y\rceil$ since $\hat{y}_{l} \neq\left\lfloor y_{l}\right\rceil$.

- $\|\hat{y}-y\|_{p} \leq\|x-y\|_{p}$ for all $x \in \mathbb{Z}^{n} \backslash\{\lfloor y\rceil\}$ :

As $x \neq\lfloor y\rceil$ there exists $k \in\{1, \ldots, n\}$ with $x_{k} \neq\left\lfloor y_{k}\right\rceil$ and hence $\left|x_{k}-y_{k}\right| \geq 1-a_{k}$ as $x_{k} \in \mathbb{Z}$. Furthermore, we have $\left|x_{j}-y_{j}\right| \geq a_{j}$ for all $j \in\{1, \ldots, n\}$ as $x_{j} \in \mathbb{Z}$.

(i) Let $p=\infty$ : since $\|x-y\|_{\infty} \geq 1-a_{k}$ and $\|\hat{y}-y\|_{\infty}=1-a_{l}$, as $\left|\hat{y}_{j}-y_{j}\right|=a_{j} \leq$ $0.5 \leq 1-a_{l}$ for all $j \neq l$, we get $\|x-y\|_{\infty} \geq 1-a_{k} \geq 1-a_{l}=\|\hat{y}-y\|_{\infty}$ as $a_{l} \geq a_{j} \forall j$.

(ii) For $p \in[1, \infty)$ we get $\|\hat{y}-y\|_{p}^{p}-\|x-y\|_{p}^{p}=\sum_{j \neq l} a_{j}^{p}+\left(1-a_{l}\right)^{p}-\sum_{j=1}^{n}\left|x_{j}-y_{j}\right|^{p}$.

If $k=l$ we have $\|\hat{y}-y\|_{p}^{p}-\|x-y\|_{p}^{p} \leq\left(1-a_{l}\right)^{p}-\left|x_{l}-y_{l}\right|^{p} \leq 0$.

Otherwise, if $k \neq l$ we have $\|\hat{y}-y\|_{p}^{p}-\|x-y\|_{p}^{p} \leq a_{k}^{p}+\left(1-a_{l}\right)^{p}-a_{l}^{p}-\left(1-a_{k}\right)^{p} \leq 0$, as $a_{k}^{p}-\left(1-a_{k}\right)^{p} \leq a_{l}^{p}-\left(1-a_{l}\right)^{p}$ since $x^{p}-(1-x)^{p}$ is monotonically increasing for $p \geq 1$ and $a_{k} \leq a_{l}$.

This means,

(i) for $p=\infty$ : $\beta_{\infty}(y)=\|\hat{y}-y\|_{\infty}-\|\lfloor y\rceil-y\|_{\infty}=1-a_{l}-a_{l}=1-2 a_{l}$

(ii) and for $p \in[1, \infty): \beta_{p}(y)=\|\hat{y}-y\|_{p}-\|\lfloor y\rceil-y\|_{p}=\sqrt[p]{\left(1-a_{l}\right)^{p}+\sum_{j \neq l} a_{j}^{p}}-\sqrt[p]{\sum_{j=1}^{n} a_{j}^{p}}$.

If we try to generalize $\beta_{p}(\bar{x})$ even further as we did with $\alpha_{p}(\bar{x})$ by taking $\min _{x \in \mathbb{R}^{n}} \beta_{p}(x)$ we observe that $\min _{x \in \mathbb{R}^{n}} \beta_{p}(x)=0$, as for example for $x=(0.5, \ldots, 0.5)$ we have $\beta_{p}(x)=0$.

We can adapt Lemma 2.15 to guarantee the Strong Rounding Property by replacing $\alpha_{p}(\bar{x})$ by $\beta_{p}(\bar{x})$ therefore we do not state it again here.

Remark 2.20. It is obvious by the definitions of $\alpha_{p}(y)$ and $\beta_{p}(y)$ that we always have $\alpha_{p}(y) \geq$ $\beta_{p}(y)$. In Figure 2.13 we marked for two different points $u$ and $v$ the points $\operatorname{Round}(x),\lfloor x\rceil, \widetilde{x}$ and $\hat{x}$ for $x \in\{u, v\}$. Furthermore, the gray area shows the set $B_{2}\left(x,\|x-\lfloor x\rceil\|_{2}\right)$ for $x \in\{u, v\}$ and the dotted circles give the size of $\alpha_{2}(x)$ and $\beta_{2}(x)$ for $x \in\{u, v\}$. 


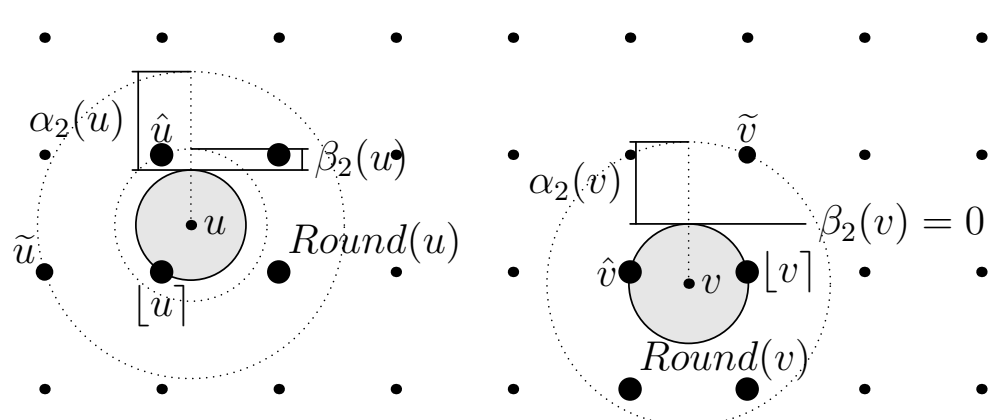

Figure 2.13.: Comparing the values of $\alpha_{2}(x)$ and $\beta_{2}(x)$ for two different points $x \in\{u, v\}$.

Remark 2.21. The fact that the level sets of a function are quasi-round (or even round) does not say anything about the actual function values. Especially it is not possible to give some bound on $f\left(x^{*}\right)-f(\bar{x})$, compare for example the function

$$
f: \mathbb{R} \rightarrow \mathbb{R}, \quad f(x)=M \cdot\left\lceil\frac{\left\|x-x^{0}\right\|_{2}}{\left\|x^{0}-\left\lfloor x^{0}\right\rceil\right\|_{2}}\right\rceil
$$

for $M \in \mathbb{R}^{+}$and $x^{0} \in \mathbb{R}^{n} \backslash \mathbb{Z}^{n}$. Obviously $\bar{x}:=\operatorname{argmin}_{x \in \mathbb{R}^{n}} f(x)=x^{0}$ with $f(\bar{x})=0$, the level sets are scaled Euclidean balls centered at $x^{0}$ and $x^{*}=\lfloor\bar{x}\rceil$. But $f\left(x^{*}\right)-f(\bar{x})=M$ can be arbitrarily large. The function described here is shown for $n=1$ in Figure 2.14.

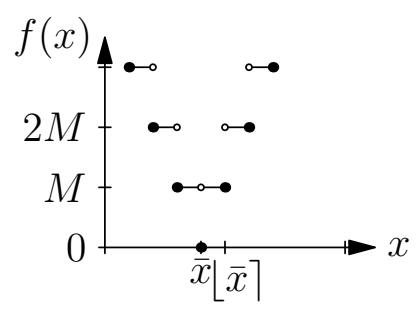

Figure 2.14.: Even if $\left|\bar{x}-x^{*}\right|<0.5$ as $x^{*}=\lfloor\bar{x}\rceil$ the difference in the function values $\mid f(\bar{x})-$ $f\left(x^{*}\right) \mid=M$ can get arbitrarily large.

This behavior does not dependent on $f$ being continuous. 


\subsection{Applications}

In this section we give some examples of functions that have quasi-round level sets as well as an application to robust optimization.

\subsubsection{Perturbed $\mathrm{p}$ norms}

Consider a $p$ norm that is perturbed by some additive noise:

$$
f: \mathbb{R}^{n} \rightarrow \mathbb{R}, \quad f(x):=\|x-a\|_{p}+\epsilon(x)
$$

where $a \in \mathbb{R}^{n}$ and $\epsilon: \mathbb{R}^{n} \rightarrow\left[0, \epsilon_{\max }\right], x \mapsto \epsilon(x)$ such that $\epsilon(a)=0$ is a small perturbance. This means, $B_{p}\left(a, z-\epsilon_{\max }\right) \subseteq \mathcal{L}_{\leq, f}(z) \subseteq B_{p}(a, z)$ for all $z \in \mathbb{R}$, i.e., $\mathcal{L}_{\leq, f}(z)$ is $(\alpha, p)$-quasi-round w.r.t. $a$ for all $\alpha \geq \epsilon_{\max }$.

Since $\epsilon(x) \geq 0$ for all $x \in \mathbb{R}^{n}$ and $\epsilon(a)=0$ we have furthermore $\bar{X}=\{a\}$ and hence if $\epsilon_{\max } \leq \alpha_{p}(a)$,

$$
\begin{array}{rll}
(I P) & \min & f(x)=\|x-a\|_{p}+\epsilon(x) \\
\text { s.t. } & x \in \mathbb{Z}^{n}
\end{array}
$$

has the Rounding Property. Furthermore, if $\epsilon_{\max } \leq \beta_{p}(a),\left(f, \mathbb{R}^{n}\right)$ even has the Strong Rounding Property. This means, if the noise is additive and positive we can investigate the (Strong) Rounding Property if we know the maximal value of the perturbance.

In the following we give three examples for realizations of $\epsilon(x)$ perturbing the Euclidean norm in $\mathbb{R}^{2}$ :

(i) $\epsilon(x)=\epsilon$ and $\epsilon \sim \mathcal{U}\left(0, \epsilon_{\max }\right), \epsilon(a)=0$, i.e., $\epsilon$ is a typical noise added to a function, compare Figure 2.15 for an example level set.

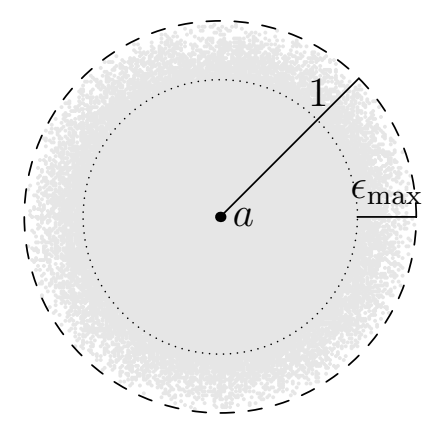

Figure 2.15.: The gray area shows the level set $\mathcal{L}_{\leq}(1)$.

(ii) $\epsilon(x)$ depends continuously on the angle between $x-a$ and a fixed axis which may be interpreted as a perturbance of the level set, compare also Figure 2.16. 


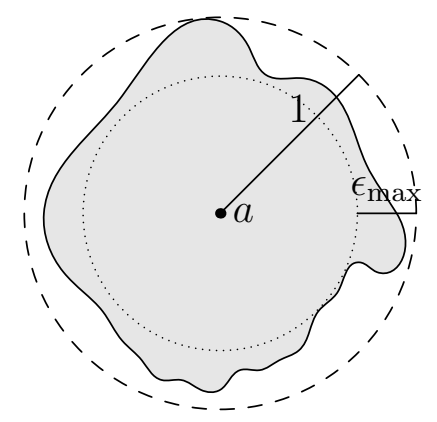

Figure 2.16.: The gray area shows the level set $\mathcal{L}_{\leq}(1)$.

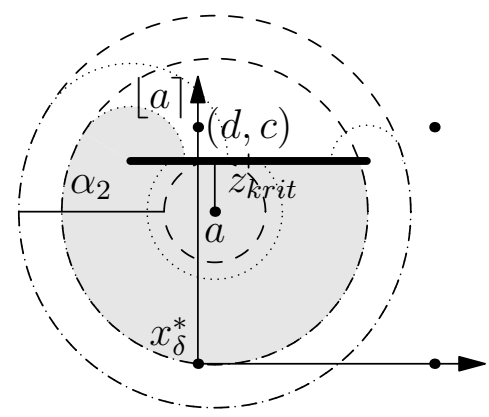

Figure 2.17.: The bold line is the barrier and the gray area shows an example level set.

(iii) Another perturbance which is often considered in location theory is the insertion of a barrier, compare Katz and Cooper [1981]. The line-shaped barrier that we consider here, as shown in Figure 2.17, is a special case of a closed polyhedron as a barrier and as we measure the distances by the Euclidean norm this is a special case of the problems considered by Butt and Cavalier [1996].

Here we consider a barrier of the form $B_{\delta}:=\left\{x \in \mathbb{R}^{2}: d-\delta \leq x_{1} \leq d+\delta, x_{2}=c\right\}$ (such that $a \notin B_{\delta}$ ). This results in

$$
f_{B_{\delta}}(x)=\left\{\begin{array}{l}
\|x-a\|_{2} \quad \text { if the way from } x \text { to } a \text { is not affected } \\
\min \left\{\|x-(d-\delta, c)\|_{2}+\|a-(d-\delta, c)\|_{2},\right. \\
\left.\|x-(d+\delta, c)\|_{2}+\|a-(d+\delta, c)\|_{2}\right\} \quad \text { otherwise }
\end{array}\right.
$$

and thus

$\epsilon(x)=\left\{\begin{array}{c}0 \quad \text { if } x_{2} \leq \max \left\{\frac{a_{2}-c}{a_{1}-(d-\delta)} x_{1}+a_{2}-a_{1} \frac{a_{2}-c}{a_{1}-(d-\delta)}, \frac{a_{2}-c}{a_{1}-(d+\delta)} x_{1}+a_{2}-a_{1} \frac{a_{2}-c}{a_{1}-(d+\delta)}\right\} \\ -\|x-a\|_{2}+\min \left\{\|x-(d-\delta, c)\|_{2}+\|a-(d-\delta, c)\|_{2},\right. \\ \left.\|x-(d+\delta, c)\|_{2}+\|a-(d+\delta, c)\|_{2}\right\} \quad \text { otherwise. }\end{array}\right.$

Hence $\epsilon(x) \geq 0$ for all $x \in \mathbb{R}^{2}$ and $\bar{X}=\{a\}$ as mentioned before. In contrast to the other examples we do not get a general $\epsilon_{\max }$ here. But we now that for $z_{k r i t}:=$ $\min _{y \in B_{\delta}}\|a-y\|_{2}$ the ball $B_{2}\left(a, z_{k r i t}\right) \subseteq \mathcal{L}_{\leq}(z)$ for all $z \geq z_{k r i t}$ (and for all $z \leq z_{k r i t}$ we 
have $\mathcal{L}_{\leq, f_{B_{\delta}}}(z)=B_{2}(a, z)$ anyway), compare again Figure 2.17. This means that the level sets are $\left(\alpha_{2}(a), 2\right)$-quasi-round w.r.t. $a$ for all $z \leq z_{k r i t}+\alpha_{2}(a)$.

\subsubsection{Strongly convex functions with Lipschitz-continuous gradient}

The following family of functions is given in a recent paper by Baes et al. [2013]: let $f$ be strongly convex and differentiable, have Lipschitz-continuous gradient and fulfill the inequalities

$$
\nabla f(y)^{t}(x-y)+\frac{l}{2}\|x-y\|_{2}^{2} \leq f(x)-f(y) \leq \nabla f(y)^{t}(x-y)+\frac{L}{2}\|x-y\|_{2}^{2} \quad \forall x, y \in \mathbb{R}^{n}
$$

for universal scalars $0 \leq l \leq L$. Assume that $\bar{x}$ is the continuous minimizer of $f$. (The minimizer is unique as $f$ is strongly convex.) Then we get $\nabla f(\bar{x})=0$ and for all $y \neq \bar{x}$

$$
\frac{l}{2}\|\bar{x}-y\|_{2}^{2} \leq f(y)-f(\bar{x}) \leq \frac{L}{2}\|\bar{x}-y\|_{2}^{2}
$$

and therefore

$$
\left\{y \in \mathbb{R}^{n}: f(y) \leq z\right\} \subseteq\left\{y \in \mathbb{R}^{n}: f(\bar{x})+\frac{l}{2}\|\bar{x}-y\|_{2}^{2} \leq z\right\}=B_{2}\left(\bar{x}, \sqrt{\frac{2}{l}(z-f(\bar{x}))}\right)
$$

and

$$
\left\{y \in \mathbb{R}^{n}: f(y) \leq z\right\} \supseteq\left\{y \in \mathbb{R}^{n}: f(\bar{x})+\frac{L}{2}\|\bar{x}-y\|_{2}^{2} \leq z\right\}=B_{2}\left(\bar{x}, \sqrt{\frac{2}{L}(z-f(\bar{x}))}\right) .
$$

This means, if $l, L$ satisfy

$$
\frac{1}{\sqrt{l}}-\frac{1}{\sqrt{L}} \leq \frac{\alpha_{2}}{\sqrt{2(f(\widetilde{x})-f(\bar{x}))}}
$$

the level sets of $f$ are $\left(\alpha_{2}, 2\right)$-quasi-round for all $z \leq f(\widetilde{x}):=\min \{f(x): x \in \operatorname{Round}(\bar{x})\}$ and thus $\left(f, \mathbb{R}^{n}\right)$ has the Rounding Property.

\subsubsection{Robust optimization}

Following the approach of Ben-Tal and Nemirovski [1998] we consider an uncertain optimization problem of the following form

$$
\begin{array}{lll}
(P(\xi)) \min & f(x, \xi) \\
\text { s.t. } & F(x, \xi) \leq 0 \\
& x \in \mathbb{R}^{n}
\end{array}
$$

where $f, F: \mathbb{R}^{n} \times \mathbb{R}^{k} \rightarrow \mathbb{R}$ with some uncertain parameter $\xi \in U$ and $U \subseteq \mathbb{R}^{k}$ is the uncertainty set: the set of possible scenarios for the parameter $\xi$.

Given such an uncertain problem the goal is to find a solution $x$ that is feasible for all possible scenarios, i.e., $F(x, \xi) \leq 0 \forall \xi \in U$ and that gives the best possible guaranteed value $\sup _{\xi \in U} f(x, \xi)$. This results in the solution of the robust counterpart of $(P(\xi))$

$$
\begin{aligned}
& (R C) \min \sup _{\xi \in U} f(x, \xi) \\
& \text { s.t. } \quad F(x, \xi) \leq 0 \quad \forall \xi \in U \\
& x \in \mathbb{R}^{n} \text {. }
\end{aligned}
$$


Again, we are interested in integer robust optimization problems and consider (2.3) with an additional integrality constraint

$$
\begin{array}{lll}
(I P(\xi)) & \min & f(x, \xi) \\
\text { s.t. } & F(x, \xi) \leq 0 \\
& x \in \mathbb{Z}^{n} .
\end{array}
$$

In the well-known book on robust integer optimization problems and its applications by Kouvelis and $\mathrm{Yu}$ [1997], the authors consider besides the robustness concept we consider here (the so called minimax or, as they call it, the absolute robust criterion) also two other concepts (robust deviation and relative robust decisions). They provide several complexity results and approaches for special (linear and nonlinear) discrete robust optimization problems. It turns out that the robust counterparts of many polynomially solvable discrete optimization problems become NP-hard. Besides that book there is of course a lot of literature on

- nonlinear robust optimization: for example the basic paper by Ben-Tal and Nemirovski [1998] we already cited, Ben-Tal and Nemirovski [2002] or Ben-Tal et al. [2012] just to mention a small and not at all complete selection. For conic quadratic and semidefinite robust optimization there is the book by Ben-Tal et al. [2009]. An overview on concepts for robust optimization and some algorithms is also given by Goerigk [2013].

- and on discrete robust optimization: which is mainly on special discrete problems such as for example Yu and Yang [1998] for robust shortest paths or Yu [1996] for robust knapsack problems. A recent survey on complexity results, approximability and algorithms for some combinatorial problems is provided by Aissi et al. [2009].

But as far as we know there is not much literature on robust discrete nonlinear optimization - probably due to the fact that robust optimization, discrete optimization and nonlinear optimization are already hard each for themselves.

Coming back to our approach, we consider the robust counterpart to problem (2.5):

$$
\begin{aligned}
& (I P R C) \quad \min \sup _{\xi \in U} f(x, \xi) \\
& \text { s.t. } F(x, \xi) \leq 0 \quad \forall \xi \in U \\
& x \in \mathbb{Z}^{n} \text {. }
\end{aligned}
$$

To solve $(I P R C)$ we investigate whether it has the (Strong) Rounding Property. This means, we assume, as before, that $(R C)$ is efficiently solvable.

Therefore we define for any fixed $\xi \in U f_{\xi}(x):=f(x, \xi)$ and for brevity's sake $F_{\xi}:=\{x \in$ $\left.\mathbb{R}^{n}: F(x, \xi) \leq 0\right\}$. Using these notations we can reformulate $(I P R C)$ as

$$
\begin{array}{ll}
\min & \hat{f}(x):=\sup _{\xi \in U} f_{\xi}(x) \\
\text { s.t. } & x \in \hat{F}:=\bigcap_{\xi \in U} F_{\xi} \\
& x \in \mathbb{Z}^{n} .
\end{array}
$$

Then we are again interested in the feasible level sets of $\hat{f}$ and we observe that

$$
\mathcal{L}_{\leq, \hat{f}}^{\hat{F}}(z)=\bigcap_{\xi \in U} F_{\xi} \cap \bigcap_{\xi \in U} \mathcal{L}_{\leq, f_{\xi}}(z)=\bigcap_{\xi \in U} \mathcal{L}_{\leq, f_{\xi}}^{F_{\xi}}(z) .
$$


This means, if $\bigcap_{\xi \in U} \mathcal{L}_{\leq, f_{\xi}}^{F_{\xi}}(z)$ is $\left(\alpha_{p}^{\prime}(\bar{x}, \hat{F}), p\right)$-quasi-round w.r.t. $\bar{x}$ for any optimal solution $\bar{x}$ to $(R C)$ Theorem 2.10 guarantees the Rounding Property.

Note that in contrast to many approaches in robust optimization our approach works for finite or continuous uncertainty sets.

We get the following result which we state and prove in the most general case we consider here. Afterwards we show some consequences and examples for special cases.

Lemma 2.22. Assume that for any optimal solution $\bar{x}$ to $(R C)$ and for any level $\hat{f}(\bar{x}) \leq z \leq$ $\min \{\hat{f}(x): x \in \operatorname{Round}(\bar{x}) \cap \hat{F}\}$ there exist $\epsilon(z), r(z), R(z) \in \mathbb{R}_{0}^{+}$such that

$$
B_{2}\left(m_{\xi}(z), r(z)\right) \subseteq \mathcal{L}_{\leq, f_{\xi}}^{F_{\xi}}(z) \subseteq B_{2}\left(m_{\xi}(z), R(z)\right)
$$

with $m_{\xi}(z) \in B_{2}(\bar{x}, \epsilon(z))$ for all $\xi \in U$ and $R(z)-r(z)+2 \epsilon(z) \leq \alpha_{2}^{\prime}(\bar{x}, \hat{F})$. Then $(I P R C)$ has the Rounding Property.

Proof. We show that for any level $z \leq \min \{\hat{f}(x): x \in \operatorname{Round}(\bar{x})\}$ the level set $\mathcal{L}_{\leq, \hat{f}}^{\hat{F}}(z)$ is $\left(\alpha_{2}^{\prime}(\bar{x}, \hat{F}), 2\right)$-quasi-round. Then Theorem 2.10 ends the proof. To this end we show that

$$
B_{2}(\bar{x}, r(z)-\epsilon(z)) \subseteq \mathcal{L}_{\leq, \hat{f}}^{\hat{F}}(z) \subseteq B_{2}(\bar{x}, R(z)+\epsilon(z)) .
$$

$B_{2}(\bar{x}, r(z)-\epsilon(z)) \subseteq \mathcal{L}_{\leq, \hat{f}}^{\hat{F}}(z):$

Let $x \in B_{2}(\bar{x}, r(z)-\epsilon(z))$. Then we have for all $\xi \in U$

$$
\left\|x-m_{\xi}(z)\right\|_{2} \leq\|x-\bar{x}\|_{2}+\left\|\bar{x}-m_{\xi}(z)\right\|_{2} \leq(r(z)-\epsilon(z))+\epsilon(z)=r(z) .
$$

Hence $x \in B_{2}\left(m_{\xi}(z), r(z)\right) \subseteq \mathcal{L}_{\leq, f_{\xi}}^{F_{\xi}}(z)$ for all $\xi \in U$ and thus $x \in \mathcal{L}_{\leq, \hat{f}}^{\hat{F}}(z)$.

The other direction follows directly since

$$
B_{2}\left(m_{\xi}(z), R(z)\right) \subseteq B_{2}(\bar{x}, R(z)+\epsilon(z)) .
$$

Since $R(z)-r(z)+2 \epsilon(z) \leq \alpha_{2}^{\prime}(\bar{x}, \hat{F})$, this means that $\mathcal{L}_{\leq, \hat{f}}^{\hat{F}}(z)$ is $\left(\alpha_{2}^{\prime}(\bar{x}, \hat{F}), 2\right)$-quasi-round.

If we assume in Lemma 2.22 that $R(z)-r(z)+2 \epsilon(z) \leq \beta_{2}^{\prime}(\bar{x}, \hat{F})$ we get that $(I P R C)$ has the Strong Rounding Property.

In the following we give some examples for Lemma 2.22. In order to keep our results readable we only consider one fixed $z \geq f(\bar{x})$ and check if the corresponding level set $\mathcal{L}_{\leq, \hat{f}}^{\hat{F}}(z)$ is quasiround. Furthermore, we set $m_{\xi}=\xi$ for all $\xi \in U$ for simplicity.

(i) Assume that for all $\xi \in U$ we have $F_{\xi}=\mathbb{R}^{n}, \mathcal{L}_{\leq, f_{\xi}}(z)=B_{2}(\xi, r(z))$ and $U \subseteq B_{2}\left(\bar{x}, \frac{\alpha_{2}(\bar{x})}{2}\right)$. Then $\mathcal{L}_{\leq, \hat{f}}(z)$ is $\left(\alpha_{2}(\bar{x}), 2\right)$-quasi-round, compare also the example in Figure 2.18.

(ii) Assume that we have $\underline{r}(z), \bar{r}(z) \in \mathbb{R}_{0}^{+}$such that $\mathcal{L}_{\leq, f_{\xi}}(z)=B_{2}\left(\xi, r_{\xi}(z)\right)$ where $\underline{r}(z) \leq$ $r_{\xi}(z) \leq \bar{r}(z)$ for all $\xi \in U$. Assume furthermore that $F_{\xi}=\mathbb{R}^{n}$ for all $\xi \in U$ and that $U \subseteq B_{2}\left(\bar{x}, \frac{\alpha_{2}(\bar{x})-\bar{r}(z)+\underline{r}(z)}{2}\right)$. Then $\mathcal{L}_{\leq, \hat{f}}(z)$ is $\left(\alpha_{2}(\bar{x}), 2\right)$-quasi-round, compare also the example in Figure 2.19. 


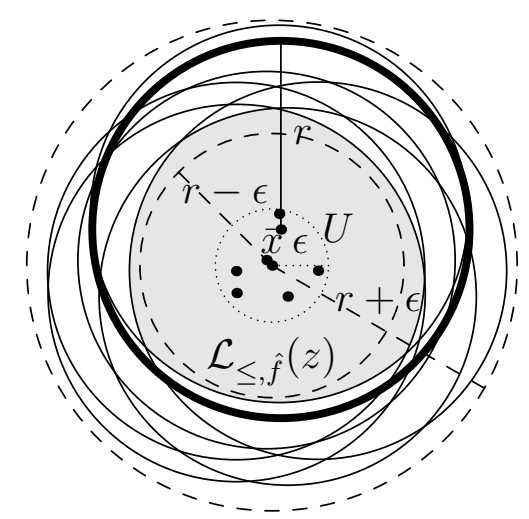

Figure 2.18.: All level sets $\mathcal{L}_{\leq, f_{\xi}}(z)$ are Euclidean balls with the same radius $r$.

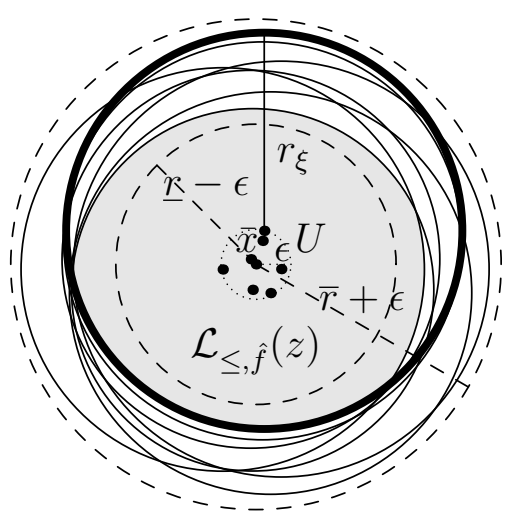

Figure 2.19.: All level sets $\mathcal{L}_{\leq, f_{\xi}}(z)$ are Euclidean balls with radius $r_{\xi}$ such that $\underline{r} \leq r_{\xi} \leq \bar{r}$.

Compared to (i) we observe that to guarantee the same $\alpha_{2}(\bar{x})$-quasi-roundness $\epsilon$ has to be smaller. Furthermore, we observe that the approximation

$$
B_{2}(\bar{x}, \underline{r}(z)-\epsilon(z)) \subseteq \mathcal{L}_{\leq, \hat{f}}(z) \subseteq B_{2}(\bar{x}, \bar{r}(z)+\epsilon(z))
$$

is on average weaker than in the former case: in (i) $B_{2}(\bar{x}, r(z)-\epsilon(z)) \subseteq \mathcal{L}_{\leq, \hat{f}}(z)$ is sharp (in the sense that $B_{2}(\bar{x}, r) \nsubseteq \mathcal{L}_{\leq, \hat{f}}(z)$ for all $\left.r>r(z)-\epsilon(z)\right)$ as soon as there exists $\xi \in U$ such that $\bar{x}=\xi$. On the other hand in (ii) $B_{2}(\bar{x}, \underline{r}(z)-\epsilon(z)) \subseteq \mathcal{L}_{\leq, \hat{f}}(z)$ is sharp if there exists $\xi \in U$ such that $\bar{x}=\xi$ and $r_{\xi}(z)=\underline{r}(z)$. The same observation is true for $B_{2}(\bar{x}, r(z)+\epsilon(z))$ or $B_{2}(\bar{x}, \bar{r}(z)+\epsilon(z))$ respectively where there has to exist $\xi \in U$ such that $\|\xi-\bar{x}\|_{2}=\epsilon(z)$ and $r_{\xi}(z)=\bar{r}(z)$ in (ii).

(iii) Assume that we have $r(z), R(z) \in \mathbb{R}_{0}^{+}$such that $B_{2}(\xi, r(z)) \subseteq \mathcal{L}_{\leq, f_{\xi}}(z) \subseteq B_{2}(\xi, R(z))$ for all $\xi \in U$. Assume furthermore that $F_{\xi}=\mathbb{R}^{n}$ for all $\xi \in U$ and that $U \subseteq$ $B_{2}\left(\bar{x}, \frac{\alpha_{2}(\bar{x})-R(z)+r(z)}{2}\right)$. Then $\mathcal{L}_{\leq, \hat{f}}(z)$ is $\left(\alpha_{2}(\bar{x}), 2\right)$-quasi-round, compare also the example in Figure 2.20.

Compared to (ii) we see that these assumptions do not impose any further constraints 


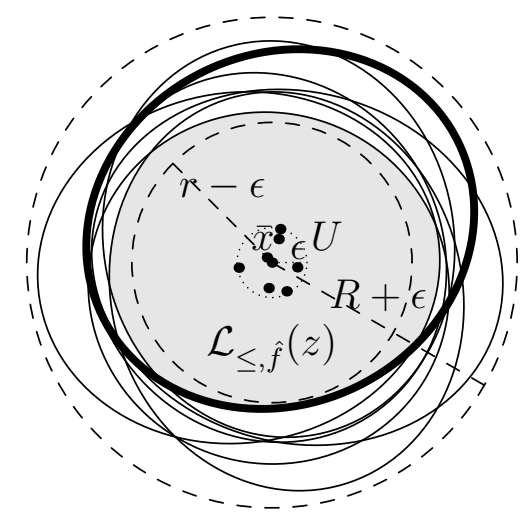

Figure 2.20.: All level sets $\mathcal{L}_{\leq, f_{\xi}}(z)$ fulfill $B_{2}(\xi, r) \subseteq \mathcal{L}_{\leq, f_{\xi}}(z) \subseteq B_{2}(\xi, R)$.

on $\epsilon(z)$ : we can keep the same size as before (setting $r(z)=\underline{r}(z)$ and $R(z)=\bar{r}(z)$ ) and also the approximation is sharp under the same assumptions as before.

\subsubsection{Quasi-round ellipsoids}

Chapter 5 is dedicated to convex quadratic functions and their level sets which are ellipsoids. Nevertheless, since it is a nice application of our concept of quasi-round sets we mention the following result here:

Whether an ellipsoid is quasi-round is determined by the difference between the length of the longest and the shortest semiaxis which in turn are determined by the eigenvalues of the descriptive matrix. This means, the ellipsoid $\left\{x \in \mathbb{R}^{n}:\left(x-x^{0}\right)^{t} Q\left(x-x^{0}\right) \leq 1\right\}$ for a positive definite matrix $Q$ is $(\alpha, 2)$-quasi-round w.r.t. $x^{0}$ if and only if $\frac{1}{\sqrt{\lambda_{\min }}}-\frac{1}{\sqrt{\lambda_{\max }}} \leq \alpha$ where $\lambda_{i}$ denote the eigenvalues of $Q$ and $\lambda_{\min }:=\min _{i} \lambda_{i}$ and $\lambda_{\max }:=\max _{i} \lambda_{i}$.

Note that we only consider quasi-roundness for $p=2$ here due to the inherent connection to ellipsoids. Further results on ellipsoids and convex quadratic functions as well as the proof of this result can be found in Chapter 5 . 


\subsection{Proximity results}

As already mentioned in Section 1.2 the (Strong) Rounding Property can also be seen as a proximity result. Using this interpretation we have shown

(i) If the level sets are $\left(\alpha_{p}^{\prime}(\bar{x}, F), p\right)$-quasi-round w.r.t. $\bar{x} \in \bar{X}$ there exists $x^{*} \in X^{*}$ such that $\left\|\bar{x}-x^{*}\right\|_{\infty}<1$.

(ii) If the level sets are $\left(\beta_{p}^{\prime}(\bar{x}, F), p\right)$-quasi-round w.r.t. $\bar{x} \in \bar{X}$ there exists $x^{*} \in X^{*}$ such that $\left\|\bar{x}-x^{*}\right\|_{\infty} \leq 0.5$.

Now we generalize these results to obtain, given $c>\frac{1}{2}$, some value $\alpha_{p}^{c}(\bar{x}, F)$ such that $\left(\alpha_{p}^{c}(\bar{x}, F), p\right)$-quasi-round level sets w.r.t. $\bar{x} \in \bar{X}$ make sure that there exists some $x^{*} \in X^{*}$ such that $\left\|\bar{x}-x^{*}\right\|_{\infty}<c$. This makes sense only for $c>\frac{1}{2}$ since for $x=(0.5, \ldots, 0.5) \in \mathbb{R}^{n}$ it is not possible to conclude $\|x-y\|_{\infty}<0.5$ for any $y \in \mathbb{Z}^{n}$.

Theorem 2.23 (Proximity result for quasi-round level sets). Let $(f, F), c>\frac{1}{2}$ be given. Assume that for any optimal solution $\bar{x}$ to $(C P)$ and for any level $f(\bar{x}) \leq z \leq \min \{f(x)$ : $\left.x \in \mathbb{Z}^{n} \cap F,\|x-\bar{x}\|_{\infty}<c\right\}$ there exists $p \in[1, \infty]$ such that the level set $\mathcal{L}_{\leq}^{F}(z)$ is $\left(\left(\alpha_{p}^{c}\right)^{\prime}(\bar{x}, F), p\right)$-quasi-round with respect to $\bar{x}$, where

$$
\left(\alpha_{p}^{c}\right)^{\prime}(\bar{x}, F):=d_{p}\left(\bar{x},\left(\mathbb{Z}^{n} \cap F\right) \backslash\left\{x:\|x-\bar{x}\|_{\infty}<c\right\}\right)-d_{p}\left(\bar{x}, \mathbb{Z}^{n}\right) .
$$

Then for each $\bar{x} \in \bar{X}$ there exists an $x^{*} \in X^{*}$ such that $\left\|x^{*}-\bar{x}\right\|_{\infty}<c$.

Again $\left(\alpha_{p}^{c}\right)^{\prime}(\bar{x}, F) \geq 0$ and hence $\left(\left(\alpha_{p}^{c}\right)^{\prime}(\bar{x}, F), p\right)$-quasi-roundness is well-defined.

Proof. (This proof is very similar to the one of Theorem 2.5. Nevertheless, we write it down here to show that the situation is basically the same.)

Let $\bar{x} \in \bar{X}$ and $x \in \mathbb{Z}^{n} \cap F$. We aim at showing that $\mathcal{L}_{\leq}^{F}(f(x)) \cap\left\{y \in \mathbb{Z}^{n}:\|y-\bar{x}\|_{\infty}<c\right\} \neq \emptyset$.

If $\min \left\{f(y): y \in \mathbb{Z}^{n} \cap F,\|y-\bar{x}\|_{\infty}<c\right\} \leq f(x)$ we are done. Otherwise we have that $x \notin\left\{y:\|y-\bar{x}\|_{\infty}<c\right\}$ and $\mathcal{L}_{\leq}^{F}(f(x))$ is $\left(\left(\alpha_{p}^{c}\right)^{\prime}(\bar{x}, F), p\right)$-quasi-round w.r.t. $\bar{x}$ and hence there exist $r, R \in \mathbb{R}_{0}^{+}$such that $B_{p}(\bar{x}, r) \subseteq \mathcal{L}_{\leq}^{F}(f(x)) \subseteq B_{p}(\bar{x}, R)$ and $R-r \leq\left(\alpha_{p}^{c}\right)^{\prime}(\bar{x}, F)$. As $d_{p}\left(\bar{x}, \mathbb{Z}^{n}\right)=\|\bar{x}-\lfloor\bar{x}\rceil\|_{p}$ we have

$$
\begin{aligned}
\|\bar{x}-\lfloor\bar{x}\rceil\|_{p} & =d_{p}\left(\bar{x},\left(\mathbb{Z}^{n} \cap F\right) \backslash\left\{y:\|y-\bar{x}\|_{\infty}<c\right\}\right)-\left(\alpha_{p}^{c}\right)^{\prime}(\bar{x}, F) \\
& \leq\|x-\bar{x}\|_{p}-\left(\alpha_{p}^{c}\right)^{\prime}(\bar{x}, F) \leq R-\left(\alpha_{p}^{c}\right)^{\prime}(\bar{x}, F) \leq r .
\end{aligned}
$$

Hence $\lfloor\bar{x}\rceil \in B_{p}(\bar{x}, r) \subseteq \mathcal{L}_{\leq}^{F}(f(x))$ and therefore $\mathbb{Z}^{n} \cap F \cap\left\{y:\|y-\bar{x}\|_{\infty}<c\right\} \neq \emptyset$ since $\|\bar{x}-\lfloor\bar{x}\rceil\|_{\infty} \leq 0.5<c$. This means that there exists $\widetilde{x} \in \operatorname{argmin}\left\{f(y): y \in \mathbb{Z}^{n} \cap F,\|y-\bar{x}\|_{\infty}<\right.$ $c\}$ and $f(\widetilde{x}) \leq f(\lfloor\bar{x}\rceil) \leq f(x)<f(\widetilde{x})$. Thus $f(x)<\min \left\{f(y): y \in \mathbb{Z}^{n} \cap F,\|y-\bar{x}\|_{\infty}<c\right\}$ leads to a contradiction.

Note that this proximity result gives us again a finite candidate set: if we know that there exists an $x^{*} \in X^{*}$ such that $\left\|x^{*}-\bar{x}\right\|_{\infty}<c$ we can solve the integer problem by solving its continuous relaxation and then comparing the objective values of the at most $(2\lceil c\rceil)^{n}$ points in $\left\{x \in \mathbb{Z}^{n}:\|x-\bar{x}\|_{\infty}<c\right\}$.

Just as before we get the following weaker formulations. 
Corollary 2.24 (Proximity result for quasi-round level sets). Let $(f, F), c>\frac{1}{2}$ be given. Assume that for any optimal solution $\bar{x}$ to $(C P)$ and for any level $f(\bar{x}) \leq z \leq \min \{f(x)$ : $\left.x \in \mathbb{Z}^{n} \cap F,\|x-\bar{x}\|_{\infty}<c\right\}$ there exists $p \in[1, \infty]$ such that the level set $\mathcal{L}_{\leq}^{F}(z)$ is $\left(\alpha_{p}^{c}(\bar{x}, F), p\right)-,\left(\alpha_{p}^{c}(\bar{x}), p\right)$ - or $\left(\alpha_{p}^{c}, p\right)$ - quasi-round with respect to $\bar{x}$, where

$$
\begin{aligned}
\alpha_{p}^{c}(\bar{x}, F) & :=d_{p}\left(\bar{x},\left(\mathbb{Z}^{n} \cap F\right) \backslash\left\{x:\|x-\bar{x}\|_{\infty}<c\right\}\right)-d_{p}\left(\bar{x}, \mathbb{Z}^{n} \cap F\right), \\
\alpha_{p}^{c}(\bar{x}) & :=d_{p}\left(\bar{x}, \mathbb{Z}^{n} \backslash\left\{x:\|x-\bar{x}\|_{\infty}<c\right\}\right)-d_{p}\left(\bar{x}, \mathbb{Z}^{n}\right) \\
\alpha_{p}^{c} & :=\min _{y \in \mathbb{R}^{n}}\left[d_{p}\left(y, \mathbb{Z}^{n} \backslash\left\{x:\|x-y\|_{\infty}<c\right\}\right)-d_{p}\left(y, \mathbb{Z}^{n}\right) .\right.
\end{aligned}
$$

Then for each $\bar{x} \in \bar{X}$ there exists an $x^{*} \in X^{*}$ such that $\left\|x^{*}-\bar{x}\right\|_{\infty}<c$.

Proof. These are direct consequences of Theorem 2.23 since $\alpha_{p}^{c}(\bar{x}, F) \leq\left(\alpha_{p}^{c}\right)^{\prime}(\bar{x}, F)$ and $\alpha_{p}^{c} \leq$ $\alpha_{p}^{c}(\bar{x}) \leq\left(\alpha_{p}^{c}\right)^{\prime}(\bar{x}, F)$.

Next we determine $\alpha_{p}^{c}(y)$.

Lemma 2.25. For a given $y \in \mathbb{R}^{n}$ and $c \in \mathbb{N}$ we define $a_{j}:=\left|\left\lfloor y_{j}\right\rceil-y_{j}\right|$ and choose $i \in$ $\operatorname{argmin}_{j=1, \ldots, n} a_{j}$. Then we get that

$$
\alpha_{p}^{c}(y):=d_{p}\left(y, \mathbb{Z}^{n} \backslash\left\{x:\|x-y\|_{\infty}<c\right\}\right)-d_{p}\left(y, \mathbb{Z}^{n}\right)
$$

is given by:

(i) for $p=\infty$ :

$$
\alpha_{\infty}^{c}(y)=c+a_{i}-\max _{j=1, \ldots, n} a_{j}
$$

(ii) for $p \in[1, \infty)$ :

$$
\alpha_{p}^{c}(y)=\sqrt[p]{\left(a_{i}+c\right)^{p}+\sum_{j \neq i} a_{j}^{p}}-\sqrt[p]{\sum_{j=1}^{n} a_{j}^{p}} .
$$

Proof. By Lemma 1.20 we have $d_{p}\left(y, \mathbb{Z}^{n}\right)=\|\lfloor y\rceil-y\|_{p}$.

For $\widetilde{y}$ with

$$
\widetilde{y}_{j}:= \begin{cases}\left\lceil y_{i}\right\rceil+c & \text { if } j=i \text { and }\left\lfloor y_{i}\right\rceil=\left\lceil y_{i}\right\rceil \\ \left\lfloor y_{i}\right\rfloor-c & \text { if } j=i \text { and }\left\lfloor y_{i}\right\rceil=\left\lfloor y_{i}\right\rfloor \\ \left\lfloor y_{j}\right\rceil & \text { if } j \neq i\end{cases}
$$

we have $\widetilde{y} \in \underset{x \in \mathbb{Z}^{n} \backslash\left\{x:\|x-y\|_{\infty}<c\right\}}{\operatorname{argmin}}\|x-y\|_{p}$ :

- Obviously $\widetilde{y} \in \mathbb{Z}^{n}$ since $c \in \mathbb{N}$

- $\|y-\widetilde{y}\|_{\infty}=\left|y_{i}-\widetilde{y}_{i}\right|=\left\{\begin{array}{ll}\left\lceil y_{i}\right\rceil+c-y_{i} & \text { if }\left\lfloor y_{i}\right\rceil=\left\lceil y_{i}\right\rceil \\ y_{i}-\left\lfloor y_{i}\right\rfloor+c & \text { if }\left\lfloor y_{i}\right\rceil=\left\lfloor y_{i}\right\rfloor\end{array}=a_{i}+c \geq c\right.$.

- $\|\widetilde{y}-y\|_{p} \leq\|x-y\|_{p}$ for all $x \in \mathbb{Z}^{n} \backslash\left\{x:\|x-y\|_{\infty}<c\right\}$ :

As $\|x-y\|_{\infty} \geq c$ there exists $k \in\{1, \ldots, n\}$ with $\left|x_{k}-y_{k}\right| \geq c$. This means, $\left|x_{k}-y_{k}\right| \geq$ $c+a_{k}$ as $x_{k} \in \mathbb{Z}$. Furthermore, we have $\left|x_{j}-y_{j}\right| \geq a_{j}$ for all $j \in\{1, \ldots, n\}$ as $x_{j} \in \mathbb{Z}$. 
(i) Let $p=\infty$ : since $\|x-y\|_{\infty} \geq c+a_{k}$ and $\|\widetilde{y}-y\|_{\infty}=c+a_{i}$ we have $\|x-y\|_{\infty} \geq$ $c+a_{k} \geq c+a_{i}=\|\widetilde{y}-y\|_{\infty}$.

(ii) For $p \in[1, \infty)$ we get $\|\widetilde{y}-y\|_{p}^{p}-\|x-y\|_{p}^{p}=\sum_{j \neq i} a_{j}^{p}+\left(c+a_{i}\right)^{p}-\sum_{j=1}^{n}\left|x_{j}-y_{j}\right|^{p}$.

If $k=i$ we have $\|\widetilde{y}-y\|_{p}^{p}-\|x-y\|_{p}^{p} \leq\left(c+a_{i}\right)^{p}-\left|x_{i}-y_{i}\right|^{p} \leq 0$.

Otherwise, if $k \neq i$ : $\|\widetilde{y}-y\|_{p}^{p}-\|x-y\|_{p}^{p} \leq a_{k}^{p}+\left(c+a_{i}\right)^{p}-a_{i}^{p}-\left(c+a_{k}\right)^{p} \leq 0$ as $a_{k}^{p}-\left(c+a_{k}\right)^{p} \leq a_{i}^{p}-\left(c+a_{i}\right)^{p}$ since $x^{p}-(c+x)^{p}$ is monotonically decreasing for $c>\frac{1}{2}, p \geq 1$ and $a_{i} \leq a_{k}$.

This means,

(i) for $p=\infty$ : $\alpha_{\infty}^{c}(y)=\|\widetilde{y}-y\|_{\infty}-\|\lfloor y\rceil-y\|_{\infty}=c+a_{i}-\max _{j=1, \ldots, n} a_{j}$

(ii) and for $p \in[1, \infty): \alpha_{p}^{c}(y)=\|\widetilde{y}-y\|_{p}-\|\lfloor y\rceil-y\|_{p}=\sqrt[p]{\left(a_{i}+c\right)^{p}+\sum_{j \neq i} a_{j}^{p}}-\sqrt[p]{\sum_{j=1}^{n} a_{j}^{p}}$.

Using this lemma, we can explicitly calculate $\alpha_{p}^{c}$.

Lemma 2.26. For $c \in \mathbb{N}$ and

(i) $p=\infty$ we have

$$
\alpha_{\infty}^{c}:=\min _{y \in \mathbb{R}^{n}} d_{\infty}\left(y, \mathbb{Z}^{n} \backslash\left\{x:\|x-y\|_{\infty}<c\right\}\right)-d_{\infty}\left(y, \mathbb{Z}^{n}\right)=c-0.5
$$

(ii) $p \in[1, \infty)$ we have

$$
\alpha_{p}^{c}:=\min _{y \in \mathbb{R}^{n}} d_{p}\left(y, \mathbb{Z}^{n} \backslash\left\{x:\|x-y\|_{\infty}<c\right\}\right)-d_{p}\left(y, \mathbb{Z}^{n}\right)=0.5 \cdot\left(\sqrt[p]{n-1+(2 c)^{p}}-\sqrt[p]{n-1}\right) .
$$

Proof. (i) Let $p=\infty$ : using Lemma 2.25 we have to solve

$$
\alpha_{\infty}^{c}=\min _{y \in \mathbb{R}^{n}}\left\{c+\min _{j=1, \ldots, n} a_{j}-\max _{j=1, \ldots, n} a_{j}\right\}
$$

where $a_{j}:=\left|\left\lfloor y_{j}\right\rceil-y_{j}\right|$.

As $0 \leq a_{j} \leq 0.5$ for all $j=1, \ldots, n$ we get $\alpha_{\infty}^{c} \geq c-0.5$. On the other hand for $y=(0.5,1, \ldots, 1) \in \mathbb{R}^{n}$ we get: $\alpha_{\infty}^{c}(y)=c-0.5$ and therefore $\alpha_{\infty}^{c} \leq c-0.5$.

(ii) Let $p \in[1, \infty)$ : using Lemma 2.25 we have to solve

$$
\alpha_{p}^{c}=\min _{y \in \mathbb{R}^{n}} \sqrt[p]{\left(a_{i}+c\right)^{p}+\sum_{j \neq i} a_{j}^{p}}-\sqrt[p]{\sum_{j=1}^{n} a_{j}^{p}}
$$


where $a_{j}:=\left|\left\lfloor y_{j}\right\rceil-y_{j}\right|$ and $i \in \operatorname{argmin}_{j=1, \ldots, n} a_{j}$, i.e.,

$$
\begin{aligned}
\alpha_{p}^{c}= & \min \sqrt[p]{\left(c+a_{i}\right)^{p}+\sum_{j \neq i} a_{j}^{p}}-\sqrt[p]{\sum_{j=1}^{n} a_{j}^{p}} \\
\text { s.t. } & 0 \leq a_{i} \leq a_{j} \leq 0.5 \quad \forall j=1, \ldots, n
\end{aligned}
$$

For $p=1$ we see directly that $\alpha_{1}=c$.

For $p>1$ : as in the proof of Lemma 2.13 we get that the optimal $a$ satisfies $a_{j}=\frac{1}{2}$ for $j \neq i$ and we are left with $\min \left\{\sqrt[p]{\left(a_{i}+c\right)^{p}+d}-\sqrt[p]{a_{i}^{p}+d}: 0 \leq a_{i} \leq \frac{1}{2}\right\}$, where $d=(n-1) / 2^{p}$. Again the minimum of the function is attained when $a_{i}=0$.

We get the following special cases: $\alpha_{p}^{1}(\bar{x}, F)=\alpha_{p}(\bar{x}, F), \alpha_{p}^{1}(\bar{x})=\alpha_{p}(\bar{x})$ and $\alpha_{p}^{1}=\alpha_{p}$. Note however that $\beta_{p}$ is not a special case of this Lemma since $\{\lfloor\bar{x}\rceil\} \neq\left\{x:\|x-\bar{x}\|_{\infty} \leq 0.5\right\}$ in general. 


\subsection{Summary}

We introduced the definition of a quasi-round set: given a fixed value $\alpha \geq 0$ and $p \in[1, \infty]$, we say that a set $M \subseteq \mathbb{R}^{n}$ is $(\alpha, p)$-quasi-round with respect to a specified center $x^{0}$, if there exist $r, R \in \mathbb{R}_{0}^{+}$where $R-r \leq \alpha$ such that $B_{p}\left(x^{0}, r\right) \subseteq M \subseteq B_{p}\left(x^{0}, R\right)$.

We are interested in quasi-round sets since we showed in Theorem 2.5 that quasi-round level sets induce the Rounding Property, if we fix $\alpha$ to the appropriate value. The geometric idea of the proof is that the choice of $\alpha$ makes sure that whenever an integer point, that is not among the rounded points, is in a level set and hence in $B_{p}(\bar{x}, R), B_{p}(\bar{x}, r)$ is large enough to contain at least one integer point and hence $\lfloor\bar{x}\rceil$. We formulated Theorem 2.5 for different values of $\alpha$ and showed the dependencies between them. If we choose $\alpha$ independently of $F$ we are able to give its value explicitly, compare Lemmas 2.11 and 2.13. Next we investigated the behavior of this $\alpha$ depending on the dimension $n$ and the parameter $p$. Then we showed some function manipulations that keep the quasi-roundness of the level sets and hence the Rounding Property.

Choosing a smaller value for $\alpha$, quasi-round level sets also guarantee the Strong Rounding Property. Hence the challenge was again to determine this value.

Section 2.3 gives some examples of problems whose level sets are quasi-round with respect to the continuous minimizer and who hence have the Rounding Property. A typical situation is the one of perturbed $p$-norms: the level sets are no longer scaled $p$-norm balls but still quasi-round, if the perturbance is not too large. We ended the section on applications by looking at robust optimization problems. Since a robust solution has to be feasible in each scenario and the objective is to minimize the worst-case objective value, the level sets of the robust counterpart are the intersections of the level sets for each scenario. Hence the question is: under which assumptions on the scenario set and on the level sets for each scenario does the robust counterpart have quasi-round level sets?

In the last section we adapted the value of $\alpha$ mentioned above to give other proximity results: as mentioned before the Rounding Property and the Strong Rounding Property can be interpreted as proximity results. Now we allow a larger value of $\alpha$ and do no longer get the Rounding Property but still a proximity result.

We collect the proximity results of this chapter in the following table: 


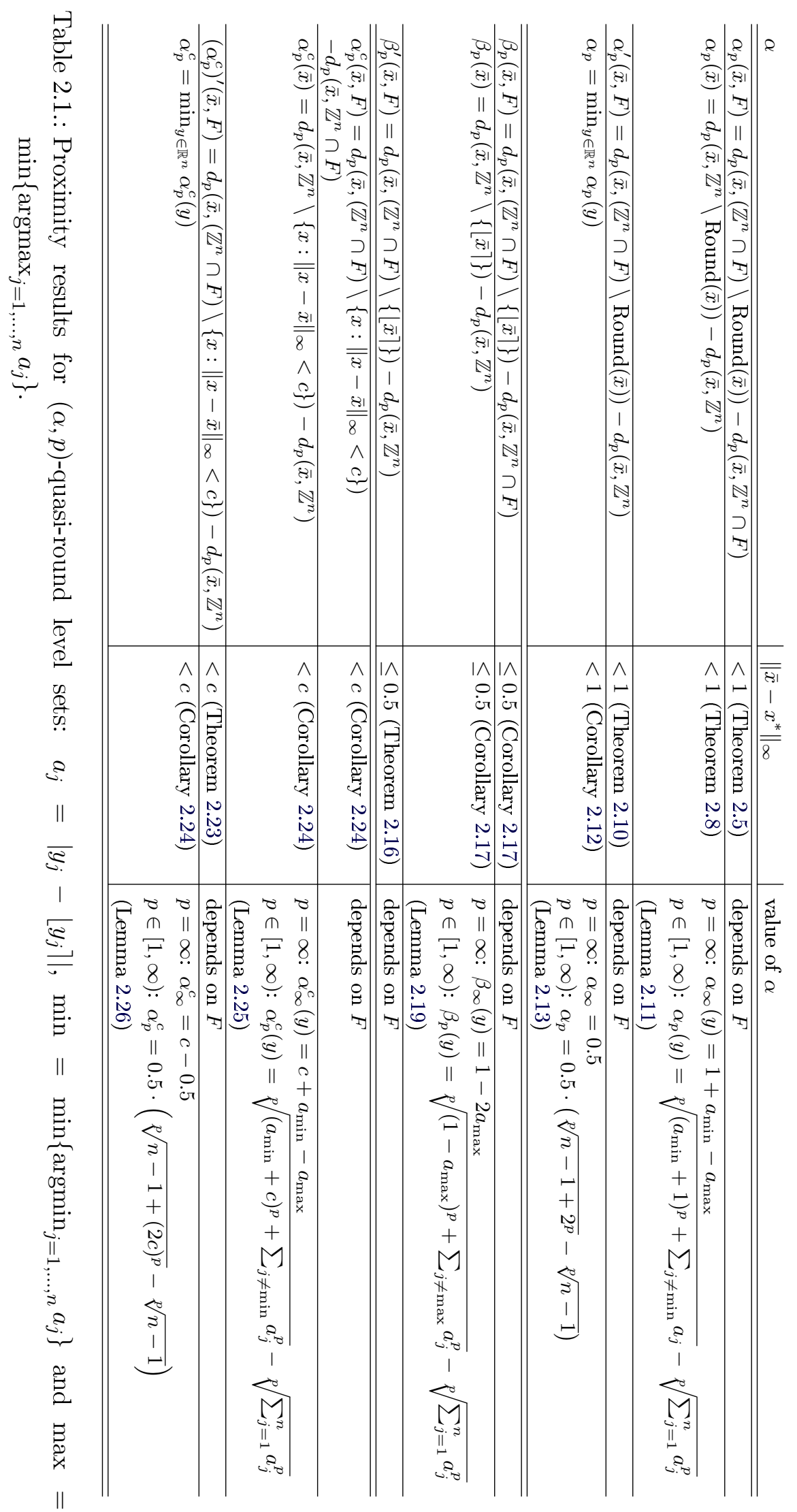




\section{Cross-shaped sets}

As mentioned before we want to make sure that whenever a feasible integer point is contained in a level set there is also a feasible rounded point (or the unique rounded point respectively) of every continuous optimal solution in this level set. One way to guarantee this is to require the level sets to be "cross-shaped".

The remainder of this chapter is organized as follows. First we define what we mean by a crossshaped set and show some basic properties. In the next section we prove that cross-shaped level sets imply the Rounding Property and investigate under which additional assumptions they also imply the Strong Rounding Property. Since the typical form of cross-shaped sets might at first glance look quite similar to biconvex sets we investigate in the next section the connection between the two definitions. Then we show some examples of functions which have cross-shaped level sets and hence the Rounding Property. We end this chapter by introducing a generalization, namely quasi-cross-shaped sets.

\subsection{Properties of cross-shaped sets}

We start by giving a basic notation we need in the following. This notation is based on the one given in Boltyanski et al. [1996], compare Remark 3.3.

Notation 3.1. Given two points $a, b \in \mathbb{R}^{n}$ we denote the 1-norm-segment given by $a$ and $b$ by

$$
[a, b]_{1}:=\left\{x \in \mathbb{R}^{n}: x_{i}=\lambda_{i} a_{i}+\left(1-\lambda_{i}\right) b_{i}, \lambda_{i} \in[0,1] \forall i=1, \ldots, n\right\} .
$$

Since we have $[a, b]_{1}=\left[a_{1}, b_{1}\right]_{1} \times \ldots \times\left[a_{n}, b_{n}\right]_{1}$ where $\left[a_{i}, b_{i}\right]_{1}=\left\{\begin{array}{l}{\left[a_{i}, b_{i}\right] \quad \text { if } a_{i} \leq b_{i}} \\ {\left[b_{i}, a_{i}\right] \quad \text { otherwise }}\end{array},[a, b]_{1}\right.$ is the box given by $a$ and $b$, see Figure 3.1 .

The basic concept in this chapter is a cross-shaped set.

Definition 3.2. A set $M \subseteq \mathbb{R}^{n}$ is called cross-shaped w.r.t. $x^{0}$ if for any $y \in M$ the 1-norm-segment $\left[x^{0}, y\right]_{1}$ is contained in $M$.

See some examples of cross-shaped sets in Figure 3.2. 


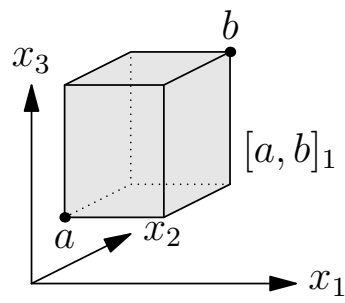

Figure 3.1.: Illustrating the definition of $[a, b]_{1}$.
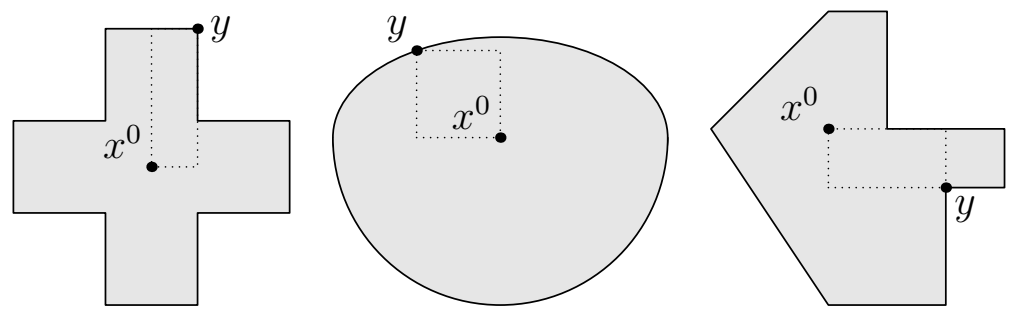

Figure 3.2.: Examples for cross-shaped sets.

Remark 3.3. (i) Compare the definition of a star-shaped set: a set $M \subseteq \mathbb{R}^{n}$ is called star-shaped if there exists a point $x^{0} \in M$, such that for any $y \in M$ the line segment $\lambda x^{0}+(1-\lambda) y, \lambda \in[0,1]$ is contained in $M$. This means that every cross-shaped set is also starshaped since $\left\{\lambda x^{0}+(1-\lambda) y: \lambda \in[0,1]\right\} \subset\left[x^{0}, y\right]_{1}$.

(ii) Both cross-shaped and star-shaped are special cases of d-star-shaped sets introduced by Boltyanski et al. [1996]: let $d: \mathbb{R}^{n} \rightarrow \mathbb{R}$ be a norm and define for $a, b \in \mathbb{R}^{n}$ the d-segment

$$
[a, b]_{d}:=\left\{x \in \mathbb{R}^{n}: d(a-x)+d(x-b)=d(a-b)\right\} .
$$

(Note that the definition of $[a, b]_{1}$ given above coincides with this definition for the 1 norm.)

Then a set $M \subseteq \mathbb{R}^{n}$ is called d-star-shaped if a point $x^{0} \in M$ exists such that for any $y \in M$ the d-segment $\left[x^{0}, y\right]_{d}$ is contained in $M$. This means that 2-star-shaped is starshaped and 1-star-shaped is cross-shaped. (Note however that we defined cross-shapedness with respect to a specified point which is a difference to the original definition.)

Whereas we show in the following that cross-shaped level sets guarantee the Rounding Property, for all other $p>1$ p-star-shaped is not sufficient for the Rounding Property.

(iii) Just as star-shaped sets, cross-shaped sets need not be convex. Nor need convex sets be cross-shaped, compare Figure 3.3: the set on the left is cross-shaped w.r.t. $x^{0}$ but not convex. The one in the middle is star-shaped w.r.t. $x^{0}$ but not cross-shaped (nor convex) and the set on the right is convex (and star-shaped w.r.t. $x^{0}$ ) but not cross-shaped w.r.t. $x^{0}$ (or any other point).

We collect some set operations that preserve the cross-shapedness.

Lemma 3.4. Let $M \subseteq \mathbb{R}^{n}$ be cross-shaped w.r.t. $x^{0} \in M$. Then the following holds: 

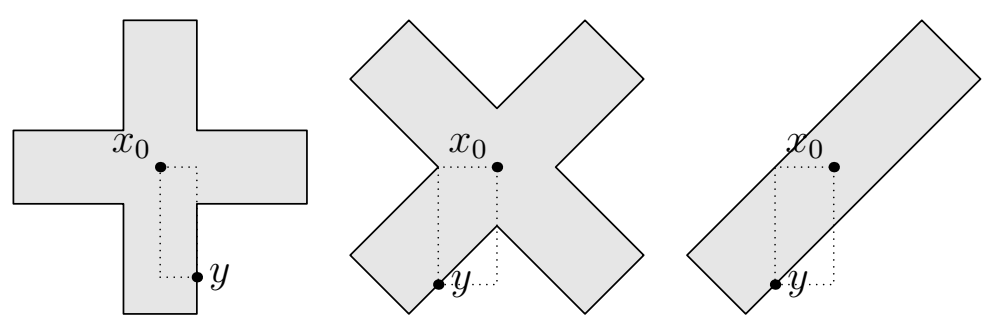

Figure 3.3.: Examples for the connections between cross-shaped, star-shaped and convex sets.

(i) $M+x$ is cross-shaped w.r.t. $x^{0}+x$ for all $x \in \mathbb{R}^{n}$.

(ii) $\lambda\left(M-x^{0}\right):=\left\{\lambda \cdot\left(x-x^{0}\right): x \in M\right\}$ is cross-shaped w.r.t. 0 for all $\lambda>0$. This means (with (i)) that $\lambda\left(M-x^{0}\right)+x^{0}$ is cross-shaped w.r.t. $x^{0}$.

(iii) Any reflection of $M$ about a hyperplane through $x^{0}$ parallel to the coordinate axes is cross-shaped w.r.t. $x^{0}$.

Let $M_{1} \subseteq \mathbb{R}^{n}$ be cross-shaped w.r.t. $x^{1} \in M_{1}$ and $M_{2} \subseteq \mathbb{R}^{n}$ be cross-shaped w.r.t. $x^{2} \in M_{2}$.

(iv) If $x^{1}=x^{2}$ we have that the intersection $M_{1} \cap M_{2}$ and the union $M_{1} \cup M_{2}$ are cross-shaped w.r.t. $x^{1}$.

(v) The Minkowski sum $M_{1}+M_{2}$ is cross-shaped w.r.t. $x^{1}+x^{2}$.

Proof. (i) This is a direct consequence of (v).

(ii) Let $y \in \lambda\left(M-x^{0}\right)$, i.e., $y=\lambda\left(x-x^{0}\right)$ for some $x \in M$. Let $p \in[0, y]_{1}$, i.e., there are $\mu_{1}, \ldots, \mu_{n} \in[0,1]$ such that

$$
\begin{aligned}
p & =\left(\mu_{1} y_{1}, \ldots, \mu_{n} y_{n}\right)=\left(\mu_{1} \lambda\left(x_{1}-x_{1}^{0}\right), \ldots, \mu_{n} \lambda\left(x_{n}-x_{n}^{0}\right)\right) \\
& =\lambda(\underbrace{\left(\mu_{1} x_{1}+\left(1-\mu_{1}\right) x_{1}^{0}, \ldots, \mu_{n} x_{n}+\left(1-\mu_{n}\right) x_{n}^{0}\right)}_{\in\left[x^{0}, x\right]_{1} \subseteq M}-x^{0}) \\
& \in \lambda \cdot\left(M-x^{0}\right) .
\end{aligned}
$$

(iii) Let $h^{j}:=\left\{x \in \mathbb{R}^{n}: x_{i}=x_{i}^{0} \forall i \neq j\right\}$ denote the hyperplane about which we reflect $M$ and $M^{j}$ the resulting set, i.e., $M^{j}$ is the set of all points $y \in \mathbb{R}^{n}$ such that there exists $x \in M$ with $y_{i}=x_{i}$ for all $i \neq j$ and $y_{j}=2 x_{j}^{0}-x_{j}$. Let $y \in M^{j}$ and consider $\left[x^{0}, y\right]_{1}=\left\{p \in \mathbb{R}^{n}: p_{k}=\lambda_{k} x_{k}^{0}+\left(1-\lambda_{k}\right) y_{k}, \lambda_{k} \in[0,1] \forall k=1, \ldots, n\right\}$. Then we have for each $p \in\left[x^{0}, y\right]_{1}$ that $p_{i}=q_{i}$ for $i \neq j$ and $p_{j}=2 x_{j}^{0}-q_{j}$ for $q_{k}=\lambda_{k} x_{k}^{0}+\left(1-\lambda_{k}\right) x_{k}$ for all $k \in\{1, \ldots, n\}$. This means $q \in\left[x^{0}, x\right]_{1} \subseteq M$ as $M$ is cross-shaped w.r.t. $x^{0}$ and hence $p \in M^{j}$. Thus $\left[x^{0}, y\right]_{1} \subseteq M^{j}$ for all $y \in M^{j}$ and hence $M^{j}$ is cross-shaped w.r.t. $x^{0}$.

(iv) Let $y \in M_{1} \cap M_{2}$, i.e., $y \in M_{i}$ for $i \in\{1,2\}$. Thus $\left[x^{1}, y\right]_{1} \subseteq M_{i}$ and hence $\left[x^{1}, y\right]_{1} \subseteq$ $M_{1} \cap M_{2}$. Analogously: if $y \in M_{1} \cup M_{2}$, there exists $k \in\{1,2\}$ such that $y \in M_{k}$. Thus $\left[x^{1}, y\right]_{1} \in M_{k}$ and hence $\left[x^{1}, y\right]_{1} \subseteq M_{1} \cup M_{2}$. 
(v) Let $y \in M_{1}+M_{2}$, i.e., $y=m^{1}+m^{2}$ for some $m^{1} \in M_{1}$ and $m^{2} \in M_{2}$. Then we get

$$
\begin{aligned}
{\left[x^{1}+x^{2}, y\right]_{1}=} & \left\{p \in \mathbb{R}^{n}: p_{k}=\lambda_{k}\left(x_{k}^{1}+x_{k}^{2}\right)+\left(1-\lambda_{k}\right)\left(m_{k}^{1}+m_{k}^{2}\right),\right. \\
& \left.\lambda_{k} \in[0,1] \forall k=1, \ldots, n\right\} \\
\subseteq & \left\{p \in \mathbb{R}^{n}: p=q^{1}+q^{2} \text { s.t. } q^{l} \in\left[x^{l}, m^{l}\right]_{1} l \in\{1,2\}\right\} \\
\subseteq & \left\{p \in \mathbb{R}^{n}: p=q^{1}+q^{2} \text { s.t. } q^{l} \in M_{l} l \in\{1,2\}\right\}=M_{1}+M_{2} .
\end{aligned}
$$

Note that (iv) is true for any index set $J$ : if all sets $M_{j}, j \in J$ are cross-shaped w.r.t. the same point $x^{0}$, also $\bigcup_{j \in J} M_{j}$ and $\bigcap_{j \in J} M_{j}$ are cross-shaped w.r.t. $x^{0}$.

Result (v) can be generalized to any finite number of sets by induction.

In general, the point $x^{0}$ with respect to whom a given set is cross-shaped is not unique. Note however that as soon as we ask for the set to be cross-shaped with respect to every point it contains, we already determine its shape, as the following lemma shows.

Lemma 3.5. Boxes are the only sets that are cross-shaped w.r.t. every point they contain.

An $n$-dimensional box is thereby a Cartesian product of $n$ 1-dimensional (maybe unbounded or open) intervals.

Proof. It is obvious that boxes are cross-shaped w.r.t. each point they contain. It remains to show the opposite direction.

Let $M \subseteq \mathbb{R}^{n}$ be a set that is cross-shaped w.r.t. each $x \in M$. Then we already know that $M$ is connected and convex.

We have to show that

$$
M=\left\{\begin{array}{cc}
{\left[\min _{x \in M} x_{1}, \max _{x \in M} x_{1}\right]} & \text { if min, max exist } \\
\left(\inf _{x \in M} x_{1}, \max _{x \in M} x_{1}\right. & \text { if only max exists } \\
{\left[\min _{x \in M} x_{1}, \sup _{x \in M} x_{1}\right)} & \text { if only min exists } \\
\left(\inf _{x \in M} x_{1}, \sup _{x \in M} x_{1}\right) & \text { otherwise }
\end{array}\right\} \times \ldots \times\left\{\begin{array}{cc}
{\left[\min _{x \in M} x_{n}, \max _{x \in M} x_{n}\right]} & \text { if min, max exist } \\
\left(\inf _{x \in M} x_{n}, \max _{x \in M} x_{n}\right] & \text { if only max exists } \\
{\left[\min _{x \in M} x_{n}, \sup _{x \in M} x_{n}\right)} & \text { if only min exists } \\
\left(\inf _{x \in M} x_{n}, \sup _{x \in M} x_{n}\right) & \text { otherwise }
\end{array}\right\}
$$

Thereby " $\subseteq$ " is obvious and it remains to be shown that each $\widetilde{x}$ where

$$
\tilde{x}_{i} \in\left\{\begin{array}{cc}
{\left[\min _{x \in M} x_{i}, \max _{x \in M} x_{i}\right]} & \text { if min, max exist } \\
\left(\inf _{x \in M} x_{i}, \max _{x \in M} x_{i}\right] & \text { if only max exists } \\
{\left[\min _{x \in M} x_{i}, \sup _{x \in M} x_{i}\right)} & \text { if only min exists } \\
\left(\inf _{x \in M} x_{i}, \sup _{x \in M} x_{i}\right) & \text { otherwise }
\end{array}\right\}
$$

is in $M$.

First we show that for each $i \in\{1, \ldots, n\}$ there exists an $x^{i} \in M$ such that $x_{i}^{i}=\widetilde{x}_{i}$ :

if $\widetilde{x}_{i} \in\left[\min _{x \in M} x_{i}, \max _{x \in M} x_{i}\right]$ there exists $\underline{x} \in M$ such that $\underline{x}_{i}=\min _{x \in M} x_{i} \leq \widetilde{x}_{i}$ and $\bar{x} \in M$ such that $\bar{x}_{i}=\max _{x \in M} x_{i} \geq \widetilde{x}_{i}$ and as $M$ is cross-shaped w.r.t $\underline{x}$ the set $[\underline{x}, \bar{x}]_{1}$ is contained in $M$ and therefore there exists an $x^{i} \in M$ such that $x_{i}^{i}=\widetilde{x}_{i}$. 
if $\widetilde{x}_{i} \in\left(\inf _{x \in M} x_{i}, \max _{x \in M} x_{i}\right]$ there exists $\underline{x} \in M$ such that $\underline{x}_{i} \leq \widetilde{x}_{i}$ : if this would not be the case it would mean that $\widetilde{x}_{i}<x_{i}$ for all $x \in M$ and this is a contradiction to $\widetilde{x}_{i}>\inf _{x \in M} x_{i}$. Analogously to the first case we get that there is a $\bar{x} \in M$ such that $\bar{x}_{i}=\max _{x \in M} x_{i} \geq \widetilde{x}_{i}$ and thus as above we get that there exists an $x^{i} \in M$ such that $x_{i}^{i}=\widetilde{x}_{i}$.

if $\widetilde{x}_{i} \in\left[\min _{x \in M} x_{i}, \sup _{x \in M} x_{i}\right)$ there exists $\underline{x} \in M$ such that $\underline{x}_{i}=\min _{x \in M} x_{i} \leq \widetilde{x}_{i}$ and $\bar{x} \in M$ such that $\bar{x}_{i} \geq \widetilde{x}_{i}$ with the same arguments as above and accordingly it follows again that there is an $x^{i} \in M$ such that $x_{i}^{i}=\widetilde{x}_{i}$.

if $\widetilde{x}_{i} \in\left(\inf _{x \in M} x_{i}, \sup _{x \in M} x_{i}\right)$ there exist $\underline{x} \in M$ and $\bar{x} \in M$ such that $\underline{x}_{i} \leq \widetilde{x}_{i} \leq \bar{x}_{i}$ by the same arguments as above and again this means, there exists $x^{i} \in M$ such that $x_{i}^{i}=\widetilde{x}_{i}$.

Choose $x^{1} \in M$ such that $x_{1}^{1}=\widetilde{x}_{1}$ and $x^{2} \in M$ such that $x_{2}^{2}=\widetilde{x}_{2}$. As $M$ is cross-shaped w.r.t. $x^{1}$ and $x^{2} \in M$ we have that $\left[x^{1}, x^{2}\right]_{1} \subseteq M$. This means that there is also an $x^{12} \in M$ where $x_{1}^{12}=x_{1}^{1}=\widetilde{x}_{1}$ and $x_{2}^{12}=x_{2}^{2}=\widetilde{x}_{2}$ and therefore $M$ is also cross-shaped w.r.t. $x^{12}$. Choose $x^{3} \in M$ such that $x_{3}^{3}=\widetilde{x}_{3}$. Then we get that $\left[x^{12}, x^{3}\right]_{1} \subseteq M$ and therefore there is an $x^{123} \in M$ where $x_{1}^{123}=x_{1}^{12}=\widetilde{x}_{1}, x_{2}^{123}=x_{2}^{12}=\widetilde{x}_{2}$ and $x_{3}^{123}=x_{3}^{3}=\widetilde{x}_{3}$. Again $M$ is cross-shaped w.r.t. $x^{123}$ and we choose $x^{4} \in M$ such that $x_{4}^{4}=\widetilde{x}_{4}$ and so on. In the end we get $x^{12 \ldots n}=\widetilde{x} \in M$.

For orthogonal rectangles, i.e., for bounded 2-dimensional boxes this result is stated in Rawlins and Wood [1988]: they call a set $M \subseteq \mathbb{R}^{2}$ box convex if for all pairs $p, q \in M$ the orthogonal rectangle defined by them lies wholly in $M$ and show that the only sets that are box convex are orthogonal rectangles. 


\subsection{Cross-shaped sets and the Rounding Property}

In this section we investigate how to use cross-shaped level sets to derive the Rounding Property.

Theorem 3.6 (Rounding Property for cross-shaped level sets.). Let $(f, F)$ be given. Assume that for any optimal solution $\bar{x}$ to $(C P)$ and for any level $f(\bar{x}) \leq z \leq \min \{f(x): x \in$ $\operatorname{Round}(\bar{x}) \cap F\}$ the level set $\mathcal{L}_{\leq}^{F}(z)$ is cross-shaped w.r.t. $\bar{x}$. Then $(f, F)$ has the Rounding Property.

Proof. Let $\bar{x} \in \bar{X}$ and $x \in \mathbb{Z}^{n} \cap F$. Using Lemma 1.18 we show that $\mathcal{L}_{\leq}^{F}(f(x)) \cap \operatorname{Round}(\bar{x}) \neq \emptyset$. If $\min \{f(u): u \in \operatorname{Round}(\bar{x}) \cap F\} \leq f(x)$ we are done. Otherwise we have that $\mathcal{L}_{\leq}^{F}(f(x))$ is cross-shaped w.r.t. $\bar{x}$.

Define $y \in \mathbb{Z}^{n}$ by $y_{i}:=\left\{\begin{array}{ll}\left\lceil\bar{x}_{i}\right\rceil & \text { if } x_{i}>\bar{x}_{i} \\ \left\lfloor\bar{x}_{i}\right\rfloor & \text { if } x_{i}<\bar{x}_{i} . \\ \bar{x}_{i} & \text { if } x_{i}=\bar{x}_{i}\end{array}\right.$ Then we have that $y \in[\bar{x}, x]_{1} \subseteq \mathcal{L}_{\leq}^{F}(f(x))$, thus $y \in \operatorname{Round}(\bar{x}) \cap F$. This means that $\operatorname{Round}(\bar{x}) \cap F \neq \emptyset$ and hence there exists $\widetilde{x} \in$ $\operatorname{argmin}\{f(u): u \in \operatorname{Round}(\bar{x}) \cap F\}$ and therefore $f(\widetilde{x}) \leq f(y) \leq f(x)<f(\widetilde{x})$. Hence $f(\widetilde{x})>f(x)$ leads to a contradiction and therefore it holds for all $x \in \mathbb{Z}^{n} \cap F$ that $f(\widetilde{x}) \leq f(x)$. Thus we have $\widetilde{x} \in \mathcal{L}_{\leq}^{F}(f(x)) \cap \operatorname{Round}(\bar{x})$.

Remark 3.7. (i) The assumption of Theorem 3.6 requires especially that the set $\bar{X}=$ $\mathcal{L}_{\leq}(f(\bar{x}))$ is cross-shaped w.r.t. every $\bar{x} \in \bar{X}$, by Lemma 3.5 this means that $\bar{X}$ has to be a (maybe degenerated) box.

(ii) It is indeed possible that $|\bar{X}|>1$ and the level sets are cross-shaped w.r.t. all points in $\bar{X}$ even if the level sets are not all boxes, compare the example in Figure 3.4: the set $\mathcal{L}_{\leq}\left(f\left(x^{*}\right)\right)$ is cross-shaped w.r.t. every $\bar{x} \in \bar{X}$ and hence $\left(f, \mathbb{R}^{2}\right)$ has the Rounding Property. We also see that the function does not have the Strong Rounding Property since for the unique integer minimizer $x^{*}$ we get $x^{*} \neq\left\lfloor\bar{x}^{2}\right\rceil$.

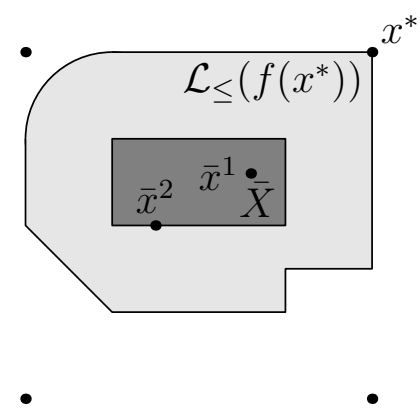

Figure 3.4.: The depicted level set is cross-shaped w.r.t. every $\bar{x} \in \bar{X}$.

Next we investigate which function manipulations keep the cross-shapedness of the level sets and therefore induce functions that have the Rounding Property. 
Lemma 3.8. (i) Let $f: \mathbb{R}^{n} \rightarrow \mathbb{R}$ and assume that for any optimal solution $\bar{x} \in \bar{X}$ to $(C P)$ and for any level $f(\bar{x}) \leq z \leq \min \{f(x): x \in \operatorname{Round}(\bar{x})\}$ the level set $\mathcal{L}_{\leq, f}(z)$ is cross-shaped w.r.t. $\bar{x}$.

a) Define $g_{1}(x):=f(x)+\gamma$ where $\gamma \in \mathbb{R}$.

b) Define $g_{2}(x):=\alpha f(x)$ where $\alpha \in \mathbb{R}, \alpha \geq 0$.

c) Let $\hat{f}: \mathbb{R} \rightarrow \mathbb{R}$ be strictly increasing and define $g_{3}(x):=\hat{f}(f(x))$.

(ii) Let $f: \mathbb{R}^{n} \rightarrow \mathbb{R}, \beta \in \mathbb{R} \backslash\{0\}, a \in \mathbb{R}^{n}$. Define $g_{4}(x):=f(\beta x-a)$. Assume that for any optimal solution $\bar{x}$ to $(C P)$ and for any level $g_{4}\left(\frac{\bar{x}+a}{\beta}\right) \leq z \leq \min \left\{g_{4}(x): x \in\right.$ $\left.\operatorname{Round}\left(\frac{\bar{x}+a}{\beta}\right)\right\}$ the level set $\mathcal{L}_{\leq, f}(z)$ is cross-shaped w.r.t. $\bar{x}$.

(iii) Let $f_{1}: \mathbb{R}^{n} \rightarrow \mathbb{R}, f_{2}: \mathbb{R}^{n} \rightarrow \mathbb{R}$ and assume that the sets of continuous minimizers coincide: $\bar{X}_{1}=\bar{X}_{2}$.

a) Assume for $i \in\{1,2\}$ : for any optimal solution $\bar{x} \in \bar{X}_{i}$ and for any level $f_{i}(\bar{x}) \leq$ $z \leq \min \left\{f_{i}(x): x \in \operatorname{Round}(\bar{x})\right\}$ the level set $\mathcal{L}_{\leq, f_{i}}(z)$ is cross-shaped w.r.t. $\bar{x}$. Define $g_{5}(x):=\min \left\{f_{1}(x), f_{2}(x)\right\}$.

b) Assume for $i \in\{1,2\}$ : for any optimal solution $\bar{x} \in \bar{X}_{i}$ and for any level $f_{i}(\bar{x}) \leq$ $z \leq \max \left\{\min \left\{f_{1}(x): x \in \operatorname{Round}(\bar{x})\right\}, \min \left\{f_{2}(x): x \in \operatorname{Round}(\bar{x})\right\}\right\}$ the level set $\mathcal{L}_{\leq, f_{i}}(z)$ is cross-shaped w.r.t. $\bar{x}$. Define $g_{6}(x):=\max \left\{f_{1}(x), f_{2}(x)\right\}$.

c) Assume for $i \in\{1,2\}$ : for any optimal solution $\bar{x} \in \bar{X}_{i}$ and for any level $z \in \mathbb{R}$ the level set $\mathcal{L}_{\leq, f_{i}}(z)$ is cross-shaped w.r.t. $\bar{x}$. Define $g_{7}(x):=f_{1}(x)+f_{2}(x)$.

Then for each $i \in\{1, \ldots, 7\}$ for any optimal solution $\bar{x} \in \min \left\{g_{i}(x): x \in \mathbb{R}^{n}\right\}$ and for any level $g_{i}(\bar{x}) \leq z \leq \min \left\{g_{i}(x): x \in \operatorname{Round}(\bar{x})\right\}$ the level set $\mathcal{L}_{\leq, g_{i}}(z)$ is cross-shaped w.r.t. $\bar{x}$ and hence $\left(g_{i}, \mathbb{R}^{n}\right)$ has the Rounding Property due to Theorem 3.6.

Proof. $g_{1}$ : Let $\bar{x} \in \operatorname{argmin}\left\{g_{1}(x): x \in \mathbb{R}^{n}\right\}$. Then $\bar{x} \in \bar{X}$ and hence for all levels $f(\bar{x}) \leq$ $z \leq \min \{f(x): x \in \operatorname{Round}(\bar{x})\}$ the level set $\mathcal{L}_{\leq, f}(z)$ is cross-shaped w.r.t. $\bar{x}$. As in the proof of Lemma 2.15, this means that the level sets $\mathcal{L}_{\leq, g_{1}}(z)$ are cross-shaped w.r.t. $\bar{x}$ for all $g_{1}(\bar{x}) \leq z \leq \min \left\{g_{1}(x): x \in \operatorname{Round}(\bar{x})\right\}$.

$g_{2}$ : if $\alpha>0$ : analogously to (i); if $\alpha=0$ we have $g_{2}(x) \equiv 0$ and hence trivially the Rounding Property.

$g_{3}:$ Let $\bar{x} \in \operatorname{argmin}\left\{g_{3}(x): x \in \mathbb{R}^{n}\right\}$. Then $\bar{x} \in \bar{X}$ and hence for all levels $f(\bar{x}) \leq z \leq$ $\min \{f(x): x \in \operatorname{Round}(\bar{x})\}$ the level set $\mathcal{L}_{\leq, f}(z)$ is cross-shaped w.r.t. $\bar{x}$.

Let $g_{3}(\bar{x}) \leq z \leq \min \left\{g_{3}(x): x \in \operatorname{Round}(\bar{x})\right\}, y \in \mathcal{L}_{\leq, g_{3}}(z)$ and $\widetilde{x} \in \operatorname{argmin}\{f(x): x \in$ $\operatorname{Round}(\bar{x})\}=\operatorname{argmin}\left\{g_{3}(x): x \in \operatorname{Round}(\bar{x})\right\}$. Then we have $g_{3}(y)=\hat{f}(f(y)) \leq z \leq$ $g_{3}(\widetilde{x})=\hat{f}(f(\widetilde{x}))$ and hence $f(y) \leq f(\widetilde{x})$. Therefore $\mathcal{L}_{\leq, f}(f(y))$ is cross-shaped w.r.t. $\bar{x}$ due to the assumption and thus $[\bar{x}, y]_{1} \subseteq \mathcal{L}_{\leq, f}(f(y))$. This means, for all $p \in[\bar{x}, y]_{1}$ we have $f(p) \leq f(y)$ and so $g_{3}(p)=\hat{f}(f(p)) \leq \hat{f}(f(y)) \leq z$ and therefore $[\bar{x}, y]_{1} \subseteq \mathcal{L}_{\leq, g_{3}}(z)$.

$g_{4}:$ Let $\bar{x} \in \operatorname{argmin}\left\{g_{4}(x): x \in \mathbb{R}^{n}\right\}$. Then $\beta \bar{x}-a \in \bar{X}$ and therefore for all $g_{4}(\bar{x}) \leq z \leq$ $\min \left\{g_{4}(x): x \in \operatorname{Round}(\bar{x})\right\}$ the level set $\mathcal{L}_{\leq, f}(z)$ is cross-shaped w.r.t. $\beta \bar{x}-a$.

Furthermore, $\mathcal{L}_{\leq, g_{4}}(z)=\frac{1}{\beta} \mathcal{L}_{\leq, f}(z)+\frac{a}{\beta}$ and hence $\mathcal{L}_{\leq, g_{4}}(z)$ is cross-shaped w.r.t. $\bar{x}$ due to Lemma 3.4. 
$g_{5}:$ Let $\bar{x} \in \operatorname{argmin}\left\{g_{5}(x): x \in \mathbb{R}^{n}\right\}$. This means that $\bar{x} \in \bar{X}_{1}=\bar{X}_{2}$ and hence $\mathcal{L}_{<, f_{1}}(z)$ is cross-shaped w.r.t. $\bar{x}$ for all $f_{1}(\bar{x}) \leq z \leq \min \left\{f_{1}(x): x \in \operatorname{Round}(\bar{x})\right\}$ and $\mathcal{L}_{\leq, f_{2}}(z)$ is cross-shaped w.r.t. $\bar{x}$ for all $f_{2}(\bar{x}) \leq z \leq \min \left\{f_{2}(x): x \in \operatorname{Round}(\bar{x})\right\}$. As in the proof of Lemma 2.15, this means that the level set $\mathcal{L}_{\leq, g_{5}}(z)$ is cross-shaped w.r.t. $\bar{x}$ for all $g_{5}(\bar{x}) \leq z \leq \min \left\{g_{5}(x): x \in \operatorname{Round}(\bar{x})\right\}$.

$g_{6}$ : Let $\bar{x} \in \operatorname{argmin}\left\{g_{6}(x): x \in \mathbb{R}^{n}\right\}$, i.e., $\bar{x} \in \bar{X}_{1}=\bar{X}_{2}$. This means that for all $z \leq$ $\max \left\{\min \left\{f_{1}(x): x \in \operatorname{Round}(\bar{x})\right\}, \min \left\{f_{2}(x): x \in \operatorname{Round}(\bar{x})\right\}\right\}$, the level sets $\mathcal{L}_{\leq, f_{1}}(z)$ and $\mathcal{L}_{\leq, f_{2}}(z)$ are cross-shaped w.r.t. $\bar{x}$. As in the proof of Lemma 2.15 this means that $\mathcal{L}_{\leq, g_{6}}(z)$ is cross-shaped w.r.t. $\bar{x}$ for all $g_{6}(\bar{x}) \leq z \leq \min \left\{g_{6}(x): x \in \operatorname{Round}(\bar{x})\right\}$.

$g_{7}$ : Let $\bar{x} \in \operatorname{argmin}\left\{g_{7}(x): x \in \mathbb{R}^{n}\right\}$. Then we have $\bar{x} \in \bar{X}_{1}=\bar{X}_{2}$. Let $z \geq g_{7}(\bar{x})$ and $y \in \mathcal{L}_{\leq, g_{7}}(z)$, i.e., $g_{7}(y)=f_{1}(y)+f_{2}(y) \leq z$.

Let $p \in[\bar{x}, y]_{1}$. Since $\mathcal{L}_{\leq, f_{1}}\left(f_{1}(y)\right)$ is cross-shaped w.r.t. $\bar{x}$ we have $p \in \mathcal{L}_{\leq, f_{1}}\left(f_{1}(y)\right)$, i.e., $f_{1}(p) \leq f_{1}(y)$ and analogously $f_{2}(p) \leq f_{2}(y)$. Together we have $g_{7}(p)=f_{1}(p)+f_{2}(p) \leq$ $f_{1}(y)+f_{2}(y) \leq z$ and hence $[\bar{x}, y]_{1} \subseteq \mathcal{L}_{\leq, g_{7}}(z)$.

We point out here that we can prove that $g_{1}, g_{2}$ and $g_{3}$ have the Rounding Property if $f$ has the Rounding Property by the observation that not only the continuous minimizers, but also the integer minimizers stay the same, but we wanted to make clear that also the cross-shapedness of the level sets is preserved.

Note that in (ii) it is not enough if $\mathcal{L}_{\leq, f}(z)$ is cross-shaped w.r.t. $\bar{x}$ for all $z \leq \min \{f(x): x \in$ Round $(\bar{x})\}$ as assumed in the first part: compare the example in Figure 3.5.

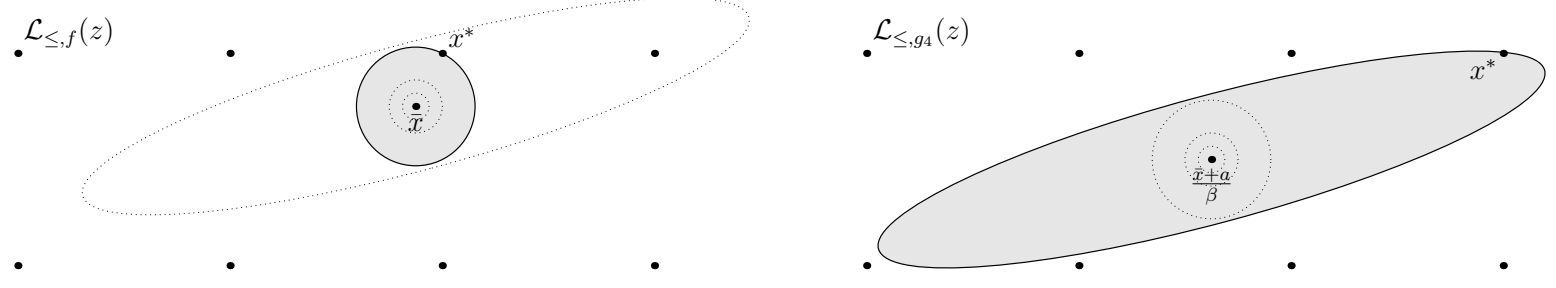

Figure 3.5.: The level sets $\mathcal{L}_{\leq, f}(z)$ are cross-shaped w.r.t. $\bar{x}$ for all $z \leq \min \{f(x): x \in$ $\operatorname{Round}(\bar{x})\}$ but not for all $z \leq \min \left\{g_{4}(x): x \in \operatorname{Round}\left(\frac{\bar{x}+\bar{a}}{\beta}\right)\right\}$ and we see that $g_{4}=f(\beta x-a)$ does not have the Rounding Property.

Also in (iii) c) we need that $\mathcal{L}_{\leq, f_{i}}(z)$ is cross-shaped w.r.t. $\bar{x}$ for all $z \in \mathbb{R}$ if we do not assume $f_{i}$ to be positive.

Note furthermore that we can generalize (iii) to any finite number of functions by induction. 
Just as with quasi-roundness also cross-shapedness does in general not imply the Strong Rounding Property, compare again Figure 3.4 for a counterexample. To also get the Strong Rounding Property we need a stronger assumption on the level sets which we define next.

Definition 3.9. As set $M \subseteq \mathbb{R}^{n}$ is called coordinate axially symmetric w.r.t. $x^{0}$ if for any $y \in M$ and for all $i \in\{1, \ldots, n\}$ it holds that the reflections of $y$ about the hyperplanes parallel to the coordinate planes and passing through $x^{0}$, i.e., $z^{i} \in \mathbb{R}^{n}$ with

$$
z_{j}^{i}:= \begin{cases}y_{j} & \text { if } j \neq i \\ 2 x_{j}^{0}-y_{j} & \text { if } j=i\end{cases}
$$

are in $M$.

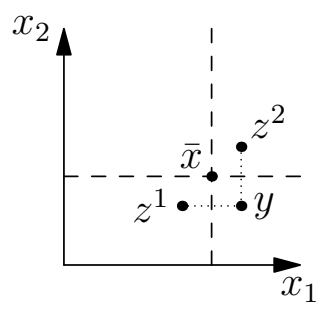

Figure 3.6.: Illustrating Definition 3.9.

We state that the set manipulations in Lemma 3.4 also preserve the coordinate axial symmetry of the sets:

Lemma 3.10. Let $M \subseteq \mathbb{R}^{n}$ be coordinate axially symmetric w.r.t. $x^{0} \in M$. Then the following holds:

(i) $M+x$ is coordinate axially symmetric w.r.t. $x^{0}+x$ for all $x \in \mathbb{R}^{n}$.

(ii) $\lambda\left(M-x^{0}\right)$ is coordinate axially symmetric w.r.t. 0 for all $\lambda>0$.

(iii) Any reflection of $M$ about a hyperplane through $x^{0}$ parallel to the coordinate axes is coordinate axially symmetric w.r.t. $x^{0}$.

Let $M_{1} \subseteq \mathbb{R}^{n}$ be coordinate axially symmetric w.r.t. $x^{1} \in M_{1}$ and $M_{2} \subseteq \mathbb{R}^{n}$ w.r.t. $x^{1} \in M_{2}$.

(iv) If $x^{1}=x^{2}$ we have that the intersection $M_{1} \cap M_{2}$ and the union $M_{1} \cup M_{2}$ are coordinate axially symmetric w.r.t. $x^{1}$.

(v) The Minkowski sum $M_{1}+M_{2}$ is coordinate axially symmetric w.r.t. $x^{1}+x^{2}$.

Proof. We use the abbreviation cas for "coordinate axially symmetric" in this proof.

(i) follows from (v) 
(ii) Let $y \in \lambda\left(M-x^{0}\right)$, i.e., there exists $m \in M$ such that $y=\lambda\left(m-x^{0}\right)$. Then we have

$$
\begin{aligned}
z^{j}:= & \left(y_{1}, \ldots, y_{j-1},-y_{j}, y_{j+1}, \ldots, y_{n}\right) \\
= & \left(\lambda\left(m_{1}-x_{1}^{0}\right), \ldots, \lambda\left(m_{j-1}-x_{j-1}^{0}\right),-\lambda\left(m_{j}-x_{j}^{0}\right),\right. \\
& \left.\lambda\left(m_{j+1}-x_{j+1}^{0}\right), \ldots, \lambda\left(m_{n}-x_{n}^{0}\right)\right) \\
= & \lambda[\underbrace{\left(m_{1}, \ldots, m_{j-1}, 2 x_{j}^{0}-m_{j}, m_{j+1}, \ldots, m_{n}\right)}_{\in M \text { since } M \text { is cas w.r.t. } x^{0}}-x^{0}] \\
& \in \lambda\left(M-x^{0}\right)
\end{aligned}
$$

for all $j \in\{1, \ldots, n\}$.

(iii) Let $h^{i}:=\left\{x \in \mathbb{R}^{n}: x_{k}=x_{k}^{0} \forall k \neq i\right\}$ denote the hyperplane about which we reflect $M$ and $M^{i}$ the resulting set. Then we have $M^{i}=M$ and hence $M^{i}$ is also cas w.r.t $x^{0}$.

(iv) Let $y \in M_{1} \cap M_{2}$, i.e., $y \in M_{i}$ for $i \in\{1,2\}$. Since $M_{i}$ is cas w.r.t. $x^{1}$ we also have that $z^{j} \in M_{i}$ and hence $z^{j} \in M_{1} \cap M_{2}$ for all $j \in\{1, \ldots, n\}$.

Let $y \in M_{1} \cup M_{2}$, i.e., $y \in M_{k}$ for some $k \in\{1,2\}$. Again since $M_{k}$ is cas w.r.t. $x^{1}$ also $z^{j} \in M_{k} \subseteq M_{1} \cup M_{2}$ for all $j \in\{1, \ldots, n\}$.

(v) Let $y \in M_{1}+M_{2}$, i.e., $y=m^{1}+m^{2}$ for some $m^{1} \in M_{1}$ and $m^{2} \in M_{2}$. Then we have

$$
\begin{aligned}
z^{k}:= & \left(y_{1}, \ldots, y_{k-1}, 2\left(x_{k}^{1}+x_{k}^{2}\right)-y_{k}, y_{k+1}, \ldots, y_{n}\right) \\
= & \begin{array}{r}
\left(m_{1}^{1}+m_{1}^{2}, \ldots, m_{k-1}^{1}+m_{k-1}^{2}, 2\left(x_{k}^{1}+x_{k}^{2}\right)-\left(m_{k}^{1}+m_{k}^{2}\right),\right. \\
\left.m_{k+1}^{1}+m_{k+1}^{2}, \ldots, m_{n}^{1}+m_{n}^{2}\right)
\end{array} \\
= & \underbrace{\left(m_{1}^{1}, \ldots, m_{k-1}^{1}, 2 x_{k}^{1}-m_{k}^{1}, m_{k+1}^{1}, \ldots, m_{n}^{1}\right)}_{\in M_{1} \text { since } M_{1} \text { is cas w.r.t. } x^{1}} \\
& \quad+\underbrace{\left(m_{1}^{2}, \ldots, m_{k-1}^{2}, 2 x_{k}^{2}-m_{k}^{2}, m_{k+1}^{2}, \ldots, m_{n}^{2}\right)}_{\in M_{2} \text { since } M_{2} \text { is cas w.r.t. } x^{2}}
\end{aligned}
$$

for all $k \in\{1, \ldots, n\}$.

Together with cross-shapedness coordinate axial symmetry guarantees the Strong Rounding Property.

Theorem 3.11 (Strong Rounding Property for cross-shaped and coordinate axially symmetric level sets.). Let $(f, F)$ be given. Assume that for any optimal solution $\bar{x}$ to $(C P)$ we have $\lfloor\bar{x}\rceil \in F$ and for any level $f(\bar{x}) \leq z \leq f(\lfloor\bar{x}\rceil)$ the level set $\mathcal{L}_{\leq}^{F}(z)$ is cross-shaped and coordinate axially symmetric w.r.t. $\bar{x}$. Then $(f, F)$ has the Strong Rounding Property.

Proof. Let $\bar{x} \in \bar{X}$ and $x \in \mathbb{Z}^{n} \cap F$. Using Lemma 1.18 we show that $\lfloor\bar{x}\rceil \in \mathcal{L}_{\leq}^{F}(f(x))$. If $f(\lfloor\bar{x}\rceil) \leq f(x)$ we are done. Otherwise we have $x \neq\lfloor\bar{x}\rceil$ and $\mathcal{L}_{\leq}^{F}(f(x))$ is cross-shaped and coordinate axially symmetric w.r.t. $\bar{x}$. 
Define $y^{1} \in \mathbb{Z}^{n}$ by

$$
y_{i}^{1}:= \begin{cases}\left\lceil\bar{x}_{i}\right\rceil & \text { if } x_{i}>\bar{x}_{i} \\ \left\lfloor\bar{x}_{i}\right\rfloor & \text { if } x_{i}<\bar{x}_{i} \\ \bar{x}_{i} & \text { if } x_{i}=\bar{x}_{i}\end{cases}
$$

Then we have $y^{1} \in \operatorname{Round}(\bar{x})$ and $y^{1} \in[\bar{x}, x]_{1} \subseteq \mathcal{L}_{\leq}^{F}(f(x))$, as in the proof of Theorem 3.6.

Define $y^{2} \in \mathbb{R}^{n}$ by

$$
y_{i}^{2}= \begin{cases}y_{i}^{1} & \text { if }\left\lfloor\bar{x}_{i}\right\rceil=y_{i}^{1} \\ 2 \bar{x}_{i}-y_{i}^{1} & \text { otherwise }\end{cases}
$$

This means that $y^{2}$ is obtained by reflecting $y^{1}$ several times along the hyperplanes through $\bar{x}$ and as $\mathcal{L}_{\leq}^{F}(f(x))$ is coordinate axially symmetric w.r.t. $\bar{x}$ and $y^{1} \in \mathcal{L}_{\leq}^{F}(f(x))$ we get that each of these reflections yields a point in $\mathcal{L}_{\leq}^{F}(f(x))$ and hence $y^{2} \in \mathcal{L}_{\leq}^{F}(f(x))$. As $\mathcal{L}_{\leq}^{F}(f(x))$ is cross-shaped w.r.t. $\bar{x}$ we know that $\left[\bar{x}, y^{2}\right]_{1} \subseteq \mathcal{L}_{\leq}^{F}(f(x))$.

Now we show that $\lfloor\bar{x}\rceil \in\left[\bar{x}, y^{2}\right]_{1} \subseteq \mathcal{L}_{\leq}^{F}(f(x))$, i.e., that $\left\lfloor\bar{x}_{i}\right\rceil \in\left\{\begin{array}{l}{\left[\bar{x}_{i}, y_{i}^{2}\right] \quad \text { if } \bar{x}_{i} \leq y_{i}^{2}} \\ {\left[y_{i}^{2}, \bar{x}_{i}\right] \quad \text { otherwise }}\end{array}\right.$ for all $i \in\{1, \ldots, n\}$.

We distinguish the following cases:

(i) if $\left\lfloor\bar{x}_{i}\right\rceil=y_{i}^{1}: y_{i}^{2}=\left\lfloor\bar{x}_{i}\right\rceil$ and we are done.

(ii) if $\left\lfloor\bar{x}_{i}\right\rceil>y_{i}^{1}: y_{i}^{2}=2 \bar{x}_{i}-y_{i}^{1}$ and $y_{i}^{1}<\bar{x}_{i}<\left\lfloor\bar{x}_{i}\right\rceil$ as both $\left\lfloor\bar{x}_{i}\right\rceil$ and $y_{i}^{1}$ are in $\operatorname{Round}\left(\bar{x}_{i}\right)$. Since we also know $\left|y_{i}^{1}-\bar{x}_{i}\right| \geq\left|\left\lfloor\bar{x}_{i}\right\rceil-\bar{x}_{i}\right|$ we get $\left\lfloor\bar{x}_{i}\right\rceil-\bar{x}_{i} \leq \bar{x}_{i}-y_{i}^{1}$. Hence we have $y_{i}^{2}=2 \bar{x}_{i}-y_{i}^{1} \geq\left\lfloor\bar{x}_{i}\right\rceil$, i.e., $\left\lfloor\bar{x}_{i}\right\rceil \in\left[\bar{x}_{i}, y_{i}^{2}\right]$.

(iii) if $\left\lfloor\bar{x}_{i}\right\rceil<y_{i}^{1}$ : analogously.

We really need cross-shaped and coordinate axially symmetric level sets since coordinate axially symmetric level sets alone are not even enough to guarantee the Rounding Property in general, compare Figure 3.7

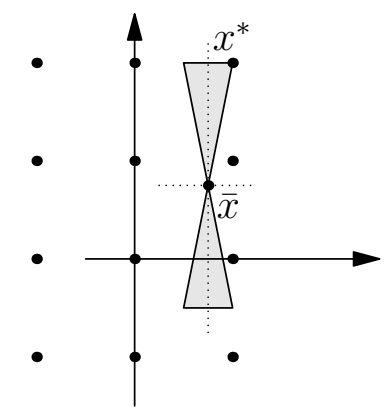

Figure 3.7.: Coordinate axially symmetric level sets do not guarantee the Rounding Property.

Next we check, as in Lemma 3.8, which function manipulations preserve the coordinate axial symmetry of the level sets and see which further functions have the Strong Rounding Property if we found one that has cross-shaped and coordinate axially symmetric level sets. 
Lemma 3.12. (i) Let $f: \mathbb{R}^{n} \rightarrow \mathbb{R}$ and assume that for any optimal solution $\bar{x}$ to $(C P)$ we have that $\lfloor\bar{x}\rceil \in F$ and for any level $f(\bar{x}) \leq z \leq f(\lfloor\bar{x}\rceil)$ the level set $\mathcal{L}_{\leq, f}(z)$ is cross-shaped and coordinate axially symmetric w.r.t. $\bar{x}$.

a) Define $g_{1}(x):=f(x)+\gamma$ where $\gamma \in \mathbb{R}$.

b) Define $g_{2}(x):=\alpha f(x)$ where $\alpha \in \mathbb{R}, \alpha \geq 0$.

c) Let $\hat{f}: \mathbb{R} \rightarrow \mathbb{R}$ be strictly increasing and define $g_{3}(x):=\hat{f}(f(x))$.

(ii) Let $f: \mathbb{R}^{n} \rightarrow \mathbb{R}, \beta \in \mathbb{R} \backslash\{0\}, a \in \mathbb{R}^{n}$. Define $g_{4}(x):=f(\beta x-a)$. Assume that for any optimal solution $\bar{x}$ to $(C P)$ we have that $\left\lfloor\frac{\bar{x}+a}{\beta}\right\rceil \in F$ and for any level $g_{4}\left(\frac{\bar{x}+a}{\beta}\right) \leq z \leq$ $g_{4}\left(\left\lfloor\frac{\bar{x}+a}{\beta}\right\rceil\right)$ the level set $\mathcal{L}_{\leq, f}(z)$ is cross-shaped and coordinate axially symmetric w.r.t. $\bar{x}$.

(iii) Let $f_{1}: \mathbb{R}^{n} \rightarrow \mathbb{R}, f_{2}: \mathbb{R}^{n} \rightarrow \mathbb{R}$ and assume that the sets of continuous minimizers coincide: $\bar{X}_{1}=\bar{X}_{2}$

a) Assume for $i \in\{1,2\}$ : for any optimal solution $\bar{x} \in \bar{X}_{i}$ we have $\lfloor\bar{x}\rceil \in F$ and for any level $f_{i}(\bar{x}) \leq z \leq f_{i}(\lfloor\bar{x}\rceil)$ the level set $\mathcal{L}_{\leq, f_{i}}(z)$ is cross-shaped and coordinate axially symmetric w.r.t. $\bar{x}$. Define $g_{5}(x):=\min \left\{f_{1}(x), f_{2}(x)\right\}$.

b) Assume for $i \in\{1,2\}$ : for any optimal solution $\bar{x} \in \bar{X}_{i}$ we have $\lfloor\bar{x}\rceil \in F$ and for any level $f_{i}(\bar{x}) \leq z \leq \max \left\{f_{1}(\lfloor\bar{x}\rceil), f_{2}(\lfloor\bar{x}\rceil)\right\}$ the level set $\mathcal{L}_{\leq, f_{i}}(z)$ is cross-shaped and coordinate axially symmetric w.r.t. $\bar{x}$. Define $g_{6}(x):=\max \left\{f_{1}(x), f_{2}(x)\right\}$.

c) Assume for $i \in\{1,2\}$ : for any optimal solution $\bar{x} \in \bar{X}_{i}$ we have $\lfloor\bar{x}\rceil \in F$ and for any level $z \geq f_{i}(\bar{x})$ the level set $\mathcal{L}_{\leq, f_{i}}(z)$ is cross-shaped and coordinate axially symmetric w.r.t. $\bar{x}$. Define $g_{7}(x):=f_{1}(x)+f_{2}(x)$.

Then for each $i \in\{1, \ldots, 7\}$ for any optimal solution $\bar{x} \in \min \left\{g_{i}(x): x \in \mathbb{R}^{n}\right\}$ we have $\lfloor\bar{x}\rceil \in F$ and for any level $g_{i}(\bar{x}) \leq z \leq g_{i}(\lfloor\bar{x}\rceil)$ the level set $\mathcal{L}_{\leq, g_{i}}(z)$ is cross-shaped and coordinate axially symmetric w.r.t. $\bar{x}$ and hence $\left(g_{i}, \mathbb{R}^{n}\right)$ has the Strong Rounding Property due to Theorem 3.11.

Proof. Again we use the abbreviation cas for "coordinate axially symmetric".

$g_{1}:$ Let $\bar{x} \in \operatorname{argmin}\left\{g_{1}(x): x \in \mathbb{R}^{n}\right\}$. Then $\bar{x} \in \bar{X}$ and hence $\lfloor\bar{x}\rceil \in F$ and for all levels $g_{1}(\bar{x}) \leq z \leq g_{1}(\lfloor\bar{x}\rceil)$ the level set $\mathcal{L}_{\leq, g_{1}}(z)$ is cross-shaped and cas w.r.t. $\bar{x}$ (as in the proof of Lemma 3.8).

$g_{2}$ : if $\alpha>0$ : analogously to (i); if $\alpha=0$ we have $g_{2}(x) \equiv 0$ and hence trivially the Strong Rounding Property.

$g_{3}$ : Let $\bar{x} \in \operatorname{argmin}\left\{g_{3}(x): x \in \mathbb{R}^{n}\right\}$. Then $\bar{x} \in \operatorname{argmin}\left\{f(x): x \in \mathbb{R}^{n}\right\}$ and hence for all levels $f(\bar{x}) \leq z \leq f(\lfloor\bar{x}\rceil)$ the level sets $\mathcal{L}_{\leq, f}(z)$ are cross-shaped and cas w.r.t. $\bar{x}$.

Let $g_{3}(\bar{x}) \leq z \leq g_{3}(\lfloor\bar{x}\rceil), y \in \mathcal{L}_{\leq, g_{3}}(z)$. Then we have (as in the proof of Lemma 3.8) that $\mathcal{L}_{\leq, f}(f(y))$ is cas w.r.t. $\bar{x}$ and hence $z^{j} \in \mathbb{R}^{n}$ defined by

$$
z_{k}^{j}:= \begin{cases}y_{k} & \text { if } k \neq j \\ 2 \bar{x}_{j}-y_{j} & \text { if } k=j\end{cases}
$$


fulfills $z^{j} \in \mathcal{L}_{\leq, f}(f(y))$, i.e., $f\left(z^{j}\right) \leq f(y) \forall j \in\{1, \ldots, n\}$. Hence $g_{3}\left(z^{j}\right)=\hat{f}\left(f\left(z^{j}\right)\right) \leq$ $\hat{f}(f(y)) \leq z$ and thus $z^{j} \in \mathcal{L}_{\leq, g_{3}}(z)$ for all $j \in\{1, \ldots, n\}$. Thus (together with Lemma 3.8) $\mathcal{L}_{\leq, g_{3}}(z)$ is cross-shaped and cas w.r.t. $\bar{x}$ for all $g_{3}(\bar{x}) \leq z \leq g_{3}(\lfloor\bar{x}\rceil)$.

The claim for $g_{4}, g_{5}, g_{6}$ follows as in Lemma 3.8 with Lemma 3.10 .

$g_{7}$ : Let $\bar{x} \in \operatorname{argmin}\left\{g_{7}(x): x \in \mathbb{R}^{n}\right\}$, i.e., $\bar{x} \in \bar{X}_{1}=\bar{X}_{2}$. Let $z \geq g_{7}(\bar{x})$. By Lemma 3.8 we know that $\mathcal{L}_{\leq, g_{7}}(z)$ is cross-shaped w.r.t. $\bar{x}$. Let $y \in \mathcal{L}_{\leq, g_{7}}(z)$. Since $\mathcal{L}_{\leq, f_{1}}(z)$ and $\mathcal{L}_{\leq, f_{2}}(z)$ are cas w.r.t. $\bar{x}$ we have that $z_{k}^{l}:=\left\{\begin{array}{ll}y_{k} & \text { if } k \neq l \\ 2 \bar{x}_{l}-y_{l} & \text { if } k=l\end{array}\right.$ fulfills $z^{l} \in \mathcal{L}_{\leq, f_{1}}\left(f_{1}(y)\right)$ and $z^{l} \in \mathcal{L}_{\leq, f_{2}}\left(f_{2}(y)\right)$, i.e., $f_{1}\left(z^{l}\right) \leq f_{1}(y)$ and $f_{2}\left(z^{l}\right) \leq f_{2}(y)$. Together we get $g_{7}\left(z^{l}\right)=$ $f_{1}\left(z^{l}\right)+f_{2}\left(z^{l}\right) \leq f_{1}(y)+f_{2}(y)=g_{7}(y) \leq z$ and hence $z^{l} \in \mathcal{L}_{\leq, g_{7}}(z)$ for all $l \in\{1, \ldots, n\}$. Hence $\mathcal{L}_{\leq, g_{7}}(z)$ is also cas w.r.t. $\bar{x}$.

Just as before we can also prove that $g_{1}, g_{2}$ and $g_{3}$ have the Strong Rounding Property by using the fact that the function manipulations do not change the continuous or integer minimizers.

We end this section by proving a sufficient and necessary condition on a function $f: \mathbb{R}^{n} \rightarrow \mathbb{R}$ that makes sure that the level sets are coordinate axially symmetric.

Lemma 3.13. Let $f: \mathbb{R}^{n} \rightarrow \mathbb{R}, \bar{x} \in \operatorname{argmin}\left\{f(x): x \in \mathbb{R}^{n}\right\}$ and $z^{0} \geq f(\bar{x})$. Then we have $\mathcal{L}_{\leq, f}(z)$ is coordinate axially symmetric w.r.t. $\bar{x}$ for all $z \leq z^{0}$

if and only if

$\forall x \in \mathcal{L}_{\leq, f}\left(z^{0}\right)$ we have $f(y)=f(x) \forall y \in \mathbb{R}^{n}$ with $\left|y_{j}-\bar{x}_{j}\right|=\left|x_{j}-\bar{x}_{j}\right| \forall j \in\{1, \ldots, n\}$,

i.e., if $f(x)$ only depends on $\left(\left|x_{1}-\bar{x}_{1}\right|, \ldots,\left|x_{n}-\bar{x}_{n}\right|\right)$.

Proof. Once again we use the abbreviation cas for "coordinate axially symmetric".

" $\Rightarrow$ ": Let $x \in \mathcal{L}_{\leq, f}\left(z^{0}\right)$, i.e., $\mathcal{L}_{\leq, f}(f(x))$ is cas w.r.t. $\bar{x}$. Let $y \in \mathbb{R}^{n}$ such that $\left|y_{j}-\bar{x}_{j}\right|=$ $\left|x_{j}-\bar{x}_{j}\right|$, i.e., $y_{j} \in\left\{x_{j}, 2 \bar{x}_{j}-x_{j}\right\}$ for all $j \in\{1, \ldots, n\}$. Thus by repeated application of the definition of cas $y \in \mathcal{L}_{\leq, f}(f(x))$ and hence $f(y) \leq f(x) \leq z^{0}$.

But this means that also $\mathcal{L}_{\leq, f}(f(y))$ is cas w.r.t. $\bar{x}$ and in the same way we get $x \in$ $\mathcal{L}_{\leq, f}(f(y))$ and hence $f(x) \leq f(y)$. Together we get $f(y)=f(x)$.

"६": Let $z \leq z^{0}$ and $y \in \mathcal{L}_{\leq, f}(z)$, i.e., also $y \in \mathcal{L}_{\leq, f}\left(z^{0}\right)$. Define $z_{k}^{j}:=\left\{\begin{array}{ll}y_{k} & \text { if } k \neq j \\ 2 \bar{x}_{j}-y_{j} & \text { if } k=j\end{array}\right.$.

Then we get for all $k=1, \ldots, n$ : $\left|z_{k}^{j}-\bar{x}_{k}\right|=\left|y_{k}-\bar{x}_{k}\right|$ and hence $f\left(z^{j}\right)=f(y) \leq z$. This means that $z^{j} \in \mathcal{L}_{\leq, f}(z)$ for all $j=1, \ldots, n$ and hence $\mathcal{L}_{\leq, f}(z)$ is cas w.r.t. $\bar{x}$.

This means that for example the level sets of $p$-norms are coordinate axially symmetric with respect to the origin. We will see more examples in Section 3.4. 


\subsection{Connection to convexity}

In this section we compare the definition of cross-shaped sets with the different variations of convexity. Besides the classical convex sets, we investigate also the following two definitions.

Definition 3.14 (Gorski et al. [2007]). Let $X \subseteq \mathbb{R}^{n_{1}}$ and $Y \subseteq \mathbb{R}^{n_{2}}$ such that $n_{1}+n_{2}=n$ be two non-empty, convex sets and let $M \subseteq X \times Y$. Define $M_{x}:=\{y \in Y:(x, y) \in M\}$ and $M_{y}:=\{x \in X:(x, y) \in M\}$. The set $M \subseteq X \times Y$ is called a biconvex set on $X \times Y$ if $M_{x}$ is convex for every $x \in X$ and $M_{y}$ is convex for every $y \in Y$.

The next definition is a generalization of the original definition given by Rawlins and Wood [1988] to $n$-dimensions.

Definition 3.15. A set $M \subseteq \mathbb{R}^{n}$ is called ortho-convex if for all $x, y \in M$ such that there exists $j \in\{1, \ldots, n\}$ with $x_{i}=y_{i}$ for all $i \neq j$ the set $[x, y]_{1}$ is contained in $M$.

We get the following connections between these three variations of convexity:

(i) If $M$ is convex it is clearly also ortho-convex (compare also Rawlins and Wood [1988]).

(ii) If $M$ is convex it is also biconvex for all possible $X, Y$, compare for example Theorem 2.2. in Gorski et al. [2007].

(iii) Even if $M$ is either biconvex for all possible $X, Y$ or ortho-convex it need not be convex, compare Figure 3.8 left.

(iv) If $n=2$ biconvexity and ortho-convexity are by definition equivalent.

(v) If $n>2$ and $M$ is ortho-convex it need not be biconvex for any $X, Y$, compare Figure 3.8 right.

(vi) If $M$ is biconvex on some $X, Y$ it is also ortho-convex, see Lemma 3.16.
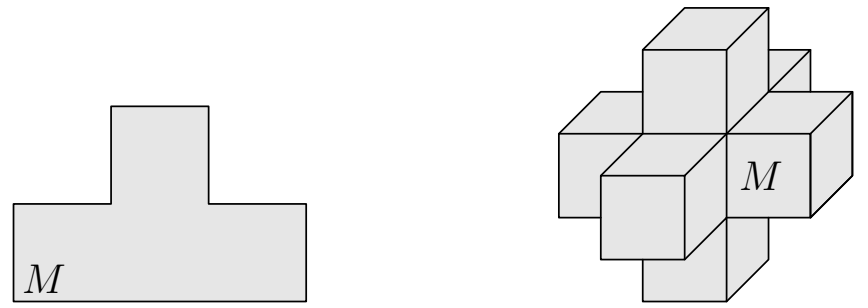

Figure 3.8.: Left: the set $M$ is biconvex and ortho-convex but not convex. Right: the set $M$ is ortho-convex but not biconvex on any $X, Y$.

Lemma 3.16. If $M$ is biconvex on some $X, Y$, then $M$ is ortho-convex.

Proof. Let $M \subseteq X \times Y$ biconvex and let $J_{x}, J_{y}$ denote the corresponding partition of the index set $\{1, \ldots, n\}$. Let $\left(x^{1}, y^{1}\right),\left(x^{2}, y^{2}\right) \in M$ with a $j \in\{1, \ldots, n\}$ such that $\left(x^{1}, y^{1}\right)_{i}=\left(x^{2}, y^{2}\right)_{i}$ for all $i \neq j$. We have to show that $\left[\left(x^{1}, y^{1}\right),\left(x^{2}, y^{2}\right)\right]_{1} \subseteq M$. 
Let us assume without loss of generality that $j \in J_{x}$ and therefore $y^{1}=y^{2}$. Let $z \in$ $\left[\left(x^{1}, y^{1}\right),\left(x^{2}, y^{2}\right)\right]_{1}$ and denote by $z_{J_{x}}$ the components of $z$ belonging to $J_{x}$. Then we have $z_{\mid J_{y}}=y^{1}, z_{i}=x_{i}^{1}$ for all $i \in J_{x} \backslash\{j\}$ and $z_{j}=\lambda\left(x^{1}, y^{1}\right)_{j}+(1-\lambda)\left(x^{2}, y^{2}\right)_{j}$ for a $\lambda \in[0,1]$.

Consider $M_{y^{1}}:=\left\{x \in X:\left(x, y^{1}\right) \in M\right\}$ : we have $x^{1}, x^{2} \in M_{y^{1}}$ and therefore also $z_{\mid J_{x}} \in M_{y^{1}}$ as $M_{y^{1}}$ convex. Hence $\left(z_{\mid J_{x}}, y^{1}\right) \in M$ and thus $z \in M$.

This means, $\left[\left(x^{1}, y^{1}\right),\left(x^{2}, y^{2}\right)\right]_{1} \subseteq M$.

Now we investigate the connections to cross-shaped. We have already seen that there are sets that are convex but not cross-shaped and sets that are cross-shaped (and even coordinate axially symmetric) that are not convex.

Lemma 3.17. Let $M \subseteq \mathbb{R}^{n}$ be cross-shaped w.r.t. some $x^{0}$, then $M$ is ortho-convex.

Proof. Let $x, y \in M, j \in\{1, \ldots, n\}$ such that $x_{i}=y_{i}$ for all $i \neq j$. Without loss of generality let $x_{j} \leq y_{j}$. We have to show that

$$
Z:=\left\{z \in \mathbb{R}^{n}: z_{i}=x_{i} \forall i, z_{j} \in\left[x_{j}, y_{j}\right]\right\} \subseteq M .
$$

If $x_{j}^{0} \leq x_{j}$ we know that $x \in\left[x^{0}, y\right]_{1}$ as $x_{i}=y_{i}$ for all $i \neq j$ and $x_{j} \in\left[x_{j}^{0}, y_{j}\right]$. This means that $Z \subseteq\left[x^{0}, y\right]_{1} \subseteq M$ since $M$ is cross-shaped w.r.t. $x^{0}$.

If $x_{j}^{0} \geq y_{j}$ we get analogously $y \in\left[x, x^{0}\right]_{1}$ and therefore $Z \subseteq\left[x, x^{0}\right]_{1} \subseteq M$.

If $x_{j} \leq x_{j}^{0} \leq y_{j}$ we have $\left[x_{j}, y_{j}\right]=\left[x_{j}, x_{j}^{0}\right] \cup\left[x_{j}^{0}, y_{j}\right]$ and therefore $Z=\left\{x \in \mathbb{R}^{n}: z_{i}=\right.$ $\left.x_{i} \forall i, z_{j} \in\left[x_{j}, x_{j}^{0}\right]\right\} \cup\left\{z \in \mathbb{R}^{n}: z_{i}=x_{i} \forall i, z_{j} \in\left[x_{j}^{0}, y_{j}\right]\right\}$ and this means, $Z \subseteq$ $\left[x, x^{0}\right]_{1} \cup\left[x^{0}, y\right]_{1} \subseteq M$.

By (iv) above, this means that for $n=2$ it holds that if $M \subseteq \mathbb{R}^{2}$ is cross-shaped w.r.t. some $x^{0}$ it is also biconvex. In general this it not the case, compare Figure 3.9. This set is obviously cross-shaped (and even coordinate axially symmetric) w.r.t. $x^{0}$ but there is no coordinate partition $X \times Y$ of the $\mathbb{R}^{3}$ such that this set is biconvex.

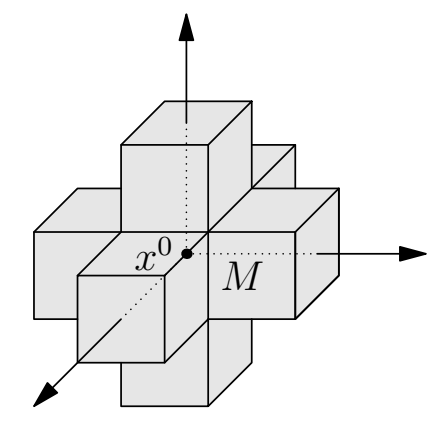

Figure 3.9.: The set $M$ is cross-shaped w.r.t. $x^{0}$ but not biconvex on any $X, Y$.

If $M$ is ortho-convex or biconvex for all $X, Y$ there need not be an $x^{0} \in M$ such that $M$ is cross-shaped w.r.t. $x^{0}$, compare the example in Figure 3.10. In fact, sets that are biconvex or ortho-convex need not be connected, but even for connected sets biconvexity or ortho-convexity is not enough to guarantee cross-shapedness. 


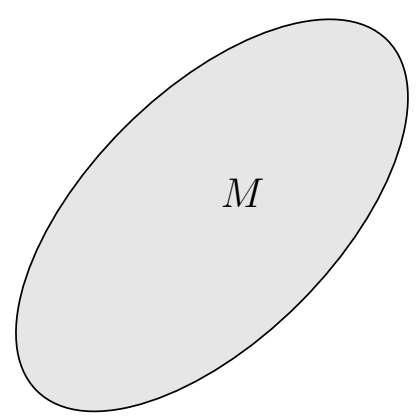

Figure 3.10.: The set $M$ is biconvex and ortho-convex but not cross-shaped w.r.t. any $x^{0}$.

A sufficient assumption to get cross-shapedness is coordinate axially symmetry and orthoconvexity.

Lemma 3.18. Let $M \subseteq \mathbb{R}^{n}$ be coordinate axially symmetric w.r.t. $x^{0}$ and ortho-convex, then it is cross-shaped w.r.t. $x^{0}$.

Proof. Let $y \in M$ and define $z \in \mathbb{R}^{n}$ by $z_{i}=2 x_{i}^{0}-y_{i}$ for all $i=1, \ldots, n$.

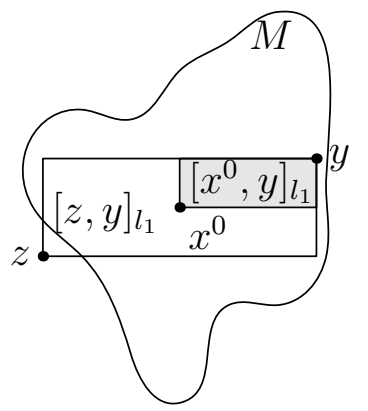

First we show $\left[y, x^{0}\right]_{1} \subseteq[y, z]_{1}$ :

Let $x \in\left[y, x^{0}\right]_{1}$, i.e., there exists $\lambda \in \mathbb{R}^{n}$ such that $x_{i}=\lambda_{i} y_{i}+\left(1-\lambda_{i}\right) x_{i}^{0}$ for all $i=1, \ldots, n$. Furthermore, $x_{i}^{0}=\frac{1}{2} y_{i}+\frac{1}{2} z_{i}$ for all $i=1, \ldots, n$.

$$
\Rightarrow \quad x_{i}=\lambda_{i} y_{i}+\left(1-\lambda_{i}\right)\left(\frac{1}{2} y_{i}+\frac{1}{2} z_{i}\right)=\left(\frac{1}{2}+\frac{\lambda_{i}}{2}\right) y_{i}+\left(\frac{1}{2}-\frac{\lambda_{i}}{2}\right) z_{i}
$$

and therefore $x \in[y, z]_{1}$ since $0 \leq \frac{1}{2}+\frac{\lambda_{i}}{2} \leq 1$.

Now we show $[y, z]_{1} \subseteq M$ and this implies that $M$ is cross-shaped w.r.t. $x^{0}$.

To this end we show first that (i) $\left[y,\left(z_{1}, y_{2}, \ldots, y_{n}\right)\right]_{1} \subseteq M$ and then for all $j=1, \ldots, n-1$ :

(ii) $\left[y,\left(z_{1}, \ldots, z_{j}, y_{j+1}, \ldots, y_{n}\right)\right]_{1} \subseteq M \quad \Rightarrow \quad\left[y,\left(z_{1}, \ldots, z_{j+1}, y_{j+2}, \ldots, y_{n}\right)\right]_{1} \subseteq M$.

This implies $[y, z]_{1} \subseteq M$.

(i) Since $M$ is cas and $y \in M$ we get $\left(z_{1}, y_{2}, \ldots, y_{n}\right) \in M$ and therefore also $\left[y,\left(z_{1}, y_{2}, \ldots, y_{n}\right)\right]_{1} \subseteq M$ as $M$ is convex in the first component, compare the assumption. 
(ii) For $x \in\left[\left(y_{1}, \ldots, y_{j}\right),\left(z_{1}, \ldots, z_{j}\right)\right]_{1}$ we have

$$
\left(x, y_{j+1}, \ldots, y_{n}\right) \in\left[y,\left(z_{1}, \ldots, z_{j}, y_{j+1}, \ldots, y_{n}\right)\right]_{1} \subseteq M
$$

and since $M$ is cas this means, $\left(x, z_{j+1}, y_{j+2}, \ldots, y_{n}\right) \in M$.

Hence $\left[\left(x, y_{j+1}, \ldots, y_{n}\right),\left(x, z_{j+1}, y_{j+2}, \ldots, y_{n}\right)\right]_{1} \subseteq M$ as $M$ is ortho-convex.

$$
\Rightarrow \quad=\quad \bigcup_{x \in\left[\left(y_{1}, \ldots, y_{j}\right),\left(z_{1}, \ldots, z_{j}\right)\right]_{1}}^{\left[y,\left(z_{1}, \ldots, z_{j+1}, y_{j+2}, \ldots, y_{n}\right)\right]_{1}}\left[\left(x, y_{j+1}, \ldots, y_{n}\right),\left(x, z_{j+1}, y_{j+2}, \ldots, y_{n}\right)\right]_{1} \subseteq M .
$$

Since biconvexity and convexity imply ortho-convexity we get the following corollary.

Corollary 3.19. (i) Let $B$ be biconvex on some $X, Y$ and axially symmetric w.r.t. $x^{0}$ then $B$ is cross-shaped w.r.t. $x^{0}$.

(ii) Let $B$ be convex and coordinate axially symmetric w.r.t. $x^{0}$ then $B$ is cross-shaped w.r.t. $x^{0}$.

The next corollary summarizes what this means for quasiconvex functions.

Corollary 3.20. Let $f: \mathbb{R}^{n} \rightarrow \mathbb{R}$ be quasiconvex. Assume that for all optimal solutions $\bar{x}$ to $(C P)$ and for all $x \in \mathcal{L}_{\leq, f}(f(\lfloor\bar{x}\rceil))$ we have that $f(y)=f(x)$ for all $y \in \mathbb{R}^{n}:\left|y_{j}-\bar{x}_{j}\right|=$ $\left|x_{j}-\bar{x}_{j}\right| \forall j \in\{1, \ldots, n\}$. Then $\left(f, \mathbb{R}^{n}\right)$ has the Strong Rounding Property.

Proof. Since $f$ is quasiconvex $\mathcal{L}_{\leq, f}(z)$ is convex for all $z \in \mathbb{R}$. The second assumption makes sure that for all optimal solutions $\bar{x}$ to $(C P)$ the level set $\mathcal{L}_{\leq, f}(z)$ is coordinate axially symmetric w.r.t. $\bar{x}$ for all $z \leq f(\lfloor\bar{x}\rceil)$, compare Lemma 3.13. Using Corollary 3.19 and Theorem 3.11 completes the proof.

We summarize the results we got in this section in Figure 3.11 .

If we assume furthermore that the set $M$ is coordinate axially symmetric w.r.t. a point $x^{0}$ we get the results shown in Figure 3.12. 


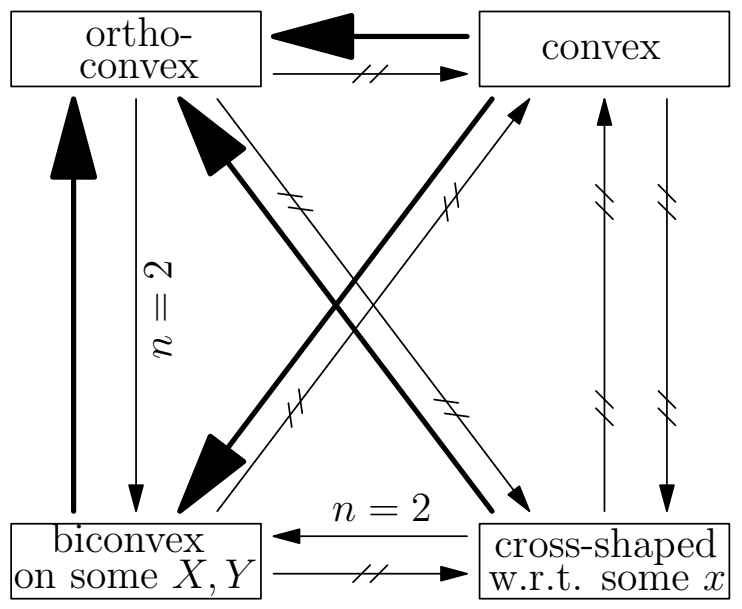

Figure 3.11.: The connections between cross-shapedness and the different variations of convexity covered in this section. The bold arrows mean implication, the thin arrows mean implication under special assumptions (in this case $n=2$ ) and the struck out arrows mean no implication.

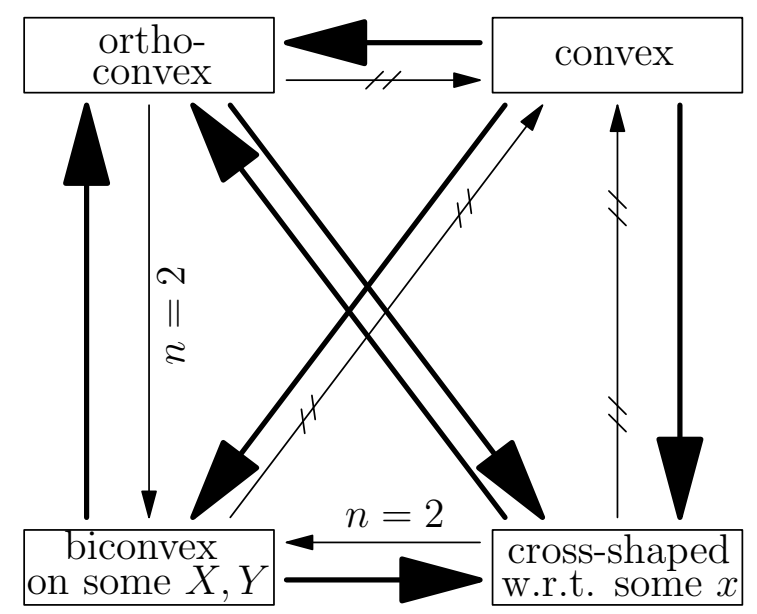

Figure 3.12.: The connections between cross-shapedness and the different variations of convexity if the set is coordinate axially symmetric with respect to some $x^{0}$. The bold arrows mean implication, the thin arrows mean implication under special assumptions (in this case $n=2$ ) and the struck out arrows mean no implication. 


\subsection{Applications}

In this section we give some examples of functions that have cross-shaped level sets and investigate for which $p \in\{1,2, \infty\}$ the median or center problem with $p$ norm has the (Strong) Rounding Property.

\subsubsection{Separable quasiconvex functions}

Similar to Hemmecke et al. [2010] we call a function $f: \mathbb{R}^{n} \rightarrow \mathbb{R}$ separable quasiconvex if it can be written as

$$
f(x)=\sum_{i=1}^{n} f_{i}\left(x_{i}\right)
$$

with quasiconvex functions $f_{i}: \mathbb{R} \rightarrow \mathbb{R}$ for all $i=1, \ldots, n$.

Lemma 3.21. The level sets of a separable quasiconvex function $f$ are cross-shaped with respect to any optimal solution $\bar{x}$ to the continuous relaxation $(C P) \min \left\{f(x): x \in \mathbb{R}^{n}\right\}$. This means, $\left(f, \mathbb{R}^{n}\right)$ has the Rounding Property.

Proof. Special case of Lemma 3.23.

We generalize $\sum_{i=1}^{n} f_{i}\left(x_{i}\right)$ as an ordered median function of the vector

$$
x^{f}:=\left(f_{1}\left(x_{1}\right), \ldots, f_{n}\left(x_{n}\right)\right) .
$$

Definition 3.22 (e.g. Puerto and Nickel [2005]). A function $g_{\lambda}: \mathbb{R}^{n} \rightarrow \mathbb{R}$ is an ordered median function, if

$$
g_{\lambda}(x)=\lambda^{t} \operatorname{sort}(x) \quad \text { for some } \lambda=\left(\lambda_{1}, \ldots, \lambda_{n}\right) \in \mathbb{R}^{n}
$$

where $\operatorname{sort}(x)$ is the sorted version of $x: \operatorname{sort}(x): \mathbb{R}^{n} \rightarrow \mathbb{R}^{n}, x \mapsto\left(x_{(1)}, x_{(2)}, \ldots, x_{(n)}\right)$ such that $x_{(1)} \leq x_{(2)} \leq \ldots \leq x_{(n)}$.

With this definition $f(x):=\sum_{i=1}^{n} f_{i}\left(x_{i}\right)=g_{(1, \ldots, 1)}\left(x^{f}\right)$.

Lemma 3.23. Let $f_{i}: \mathbb{R} \rightarrow \mathbb{R}$ be quasiconvex functions and $\lambda_{i} \geq 0$ for all $i=1, \ldots, n$. Then the level sets of $g_{\lambda}\left(x^{f}\right)$ are cross-shaped with respect to any optimal solution $\bar{x}$ to the continuous relaxation $\min \left\{g_{\lambda}\left(x^{f}\right): x \in \mathbb{R}^{n}\right\}$. This means, $\left(g_{\lambda}\left(x^{f}\right), \mathbb{R}^{n}\right)$ has the Rounding Property.

Proof. We use the following notation: $\operatorname{sort}(x)=:\left(x_{(1)_{x}}, x_{(2)_{x}}, \ldots, x_{(n)_{x}}\right)$ and $\operatorname{sort}(y)=$ : $\left(y_{(1)_{y}}, \ldots, y_{(n)_{y}}\right)$ to make clear that the two vectors are ordered in different ways.

(i) First we show that if $a=\left(a_{1}, \ldots, a_{n}\right)$ and $b=\left(b_{1}, \ldots, b_{n}\right)$ such that $a_{i} \leq b_{i}$ for all $i=1, \ldots, n$ we have $a_{(i)_{a}} \leq b_{(i)_{b}}$ for all $i=1, \ldots, n$. 
We show the claim by mathematical induction:

Base Case: $b_{(1)_{b}}=b_{k}$ for some $k \in\{1, \ldots, n\}$ and therefore: $b_{(1)_{b}}=b_{k} \geq a_{k} \geq$ $a_{(1)_{a}}$.

Inductive Step: $j \rightarrow j+1$ : assume $a_{(j+1)_{a}}>b_{(j+1)_{b}}$. This means, $a_{(k)_{a}}>$ $b_{(j+1)_{b}}$ for all $k=j+1, \ldots, n$ and therefore $b_{(j+1)_{b}}<a_{(k)_{a}} \leq b_{(k)_{a}} \forall k=$ $j+1, \ldots, n$. Hence there are $n-j$ entries in $b$ that are greater than $b_{(j+1)_{b}}$, which is a contradiction to the definition of $b_{(j+1)_{b}}$.

(ii) Let $\bar{x}$ be an optimal solution to $\min \left\{g_{\lambda}\left(x^{f}\right): x \in \mathbb{R}^{n}\right\}$. We show that this means that $f_{k}\left(\bar{x}_{k}\right) \leq f_{k}(x)$ for all $x \in \mathbb{R}$ and for all $k=1, \ldots, n$.

Assume that there is a $k \in\{1, \ldots, n\}$ and an $x \in \mathbb{R}$ such that $f_{k}(x)<f_{k}\left(\bar{x}_{k}\right)$. Define $y:=\left(\bar{x}_{1}, \ldots, \bar{x}_{k-1}, x, \bar{x}_{k+1}, \ldots, \bar{x}_{n}\right)$. Then we have $f_{i}\left(y_{i}\right) \leq f_{i}\left(\bar{x}_{i}\right)$ for all $i=1, \ldots, n$. But this means (using (i)), $f_{(i)_{f(y)}}\left(y_{(i)_{f(y)}}\right) \leq f_{(i)_{f(\bar{x})}}\left(\bar{x}_{(i)_{f(\bar{x})}}\right)$ for all $i=1, \ldots, n$ and since $f_{(i)_{f(y)}}\left(y_{(i)_{f(y)}}\right)=f_{(i)_{f(\bar{x})}}\left(\bar{x}_{(i)_{f(\bar{x})}}\right)$ for all $i=1, \ldots, n$ would mean $f_{k}\left(\bar{x}_{k}\right)=f_{k}\left(y_{k}\right)$ we know that there is at least one $j \in\{1, \ldots, n\}$ such that $f_{(j)_{f(y)}}\left(y_{(j)_{f(y)}}\right)<f_{(j)_{f(\bar{x})}}\left(\bar{x}_{(j)_{f(\bar{x})}}\right)$. Hence

$$
\sum_{i=1}^{n} \lambda_{i} f_{(i)_{f(y)}}\left(y_{(i)_{f(y)}}\right)<\sum_{i=1}^{n} \lambda_{i} f_{(i)_{f(\bar{x})}}\left(\bar{x}_{(i)_{f(\bar{x})}}\right)
$$

as $\lambda_{i} \geq 0$, which is a contradiction to $\bar{x}$ being an optimal solution to $\min \left\{g_{\lambda}\left(x^{f}\right): x \in \mathbb{R}^{n}\right\}$.

(iii) Let $z \geq g_{\lambda}\left(\bar{x}^{f}\right)$. We show that the corresponding level set is cross-shaped w.r.t. $\bar{x}$. If $\mathcal{L}_{\leq}(z)=\{\bar{x}\}$ we are done. Therefore assume that there is another point $y \in \mathcal{L}_{\leq}(z)$. Then every point $x \in[\bar{x}, y]_{1}$ can be described as $x=\left(\mu_{1} \bar{x}_{1}+\left(1-\mu_{1}\right) y_{1}, \ldots, \mu_{n} \bar{x}_{n}+\left(1-\mu_{n}\right) y_{n}\right)$ with $\mu_{i} \in[0,1]$ for all $i=1, \ldots, n$. This means,

$$
x^{f}=\left(f_{1}\left(\mu_{1} \bar{x}_{1}+\left(1-\mu_{1}\right) y_{1}\right), \ldots, f_{n}\left(\mu_{n} \bar{x}_{n}+\left(1-\mu_{n}\right) y_{n}\right)\right)
$$

and

$$
\begin{aligned}
f_{i}\left(\mu_{i} \bar{x}_{i}+\left(1-\mu_{i}\right) y_{i}\right) & \leq \max \left\{f_{i}\left(\bar{x}_{i}\right), f_{i}\left(y_{i}\right)\right\} \quad \text { as } f_{i} \text { quasiconvex } \\
& =f_{i}\left(y_{i}\right) \quad \text { compare (ii) }
\end{aligned}
$$

Using (i) we get $f_{(i)_{f(x)}}\left(x_{(i)_{f(x)}}\right) \leq f_{(i)_{f(y)}}\left(y_{(i)_{f(y)}}\right)$ and therefore

$$
\begin{aligned}
g_{\lambda}\left(x^{f}\right) & =\sum_{i=1}^{n} \lambda_{i} f_{(i)_{f(x)}}\left(x_{(i)_{f(x)}}\right) \\
& \leq \sum_{i=1}^{n} \lambda_{i} f_{(i)_{f(y)}}\left(y_{(i)_{f(y)}}\right) \quad \text { as } \lambda_{i} \geq 0 \\
& =g_{\lambda}\left(y^{f}\right) \leq z .
\end{aligned}
$$

So we have $x \in \mathcal{L}_{\leq}(z)$ and thus $[\bar{x}, y]_{1} \subseteq \mathcal{L}_{\leq}(z)$ for every $y \in \mathcal{L}_{\leq}(z)$. Summarizing, $\mathcal{L}_{\leq}(z)$ is cross-shaped w.r.t. $\bar{x}$ for all $z \geq g_{\lambda}\left(\bar{x}^{f}\right)$. 
Example 3.24. Let $f_{1}: \mathbb{R} \rightarrow \mathbb{R}, x \mapsto x^{2}$ and $f_{2}: \mathbb{R} \rightarrow \mathbb{R}, x \mapsto|x|$. Then we consider $\lambda \in\left\{(1,1),(1,2),(2,1),(-1,1)\right.$ and depict the resulting level sets $\mathcal{L}_{\leq, g_{\lambda^{j}}\left(x^{f}\right)}(1)$ for $j=1, \ldots, 4$ in Figure 3.13 .
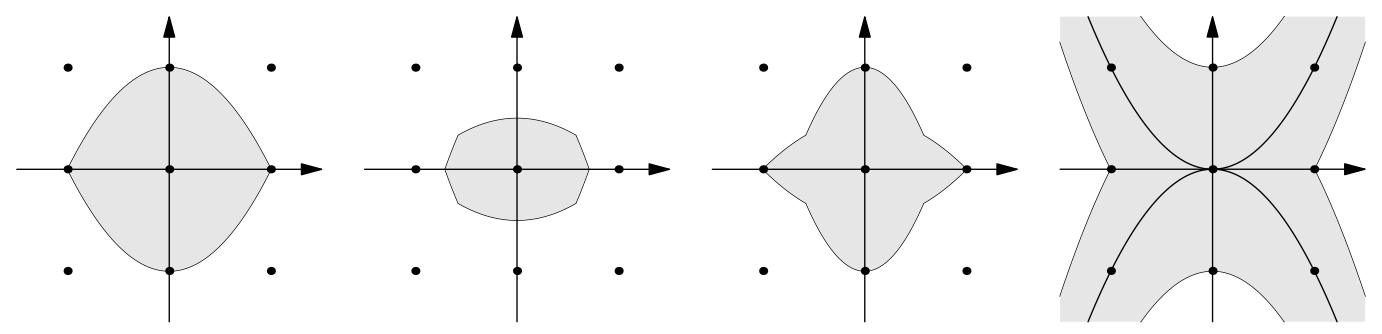

Figure 3.13.: The level sets $\mathcal{L}_{\leq, g_{\lambda^{j}}\left(x^{f}\right)}(1)$ for $\lambda^{1}=(1,1), \lambda^{2}=(1,2), \lambda^{3}=(2,1)$ and $\lambda^{4}=$ $(-1,1)$.

We see that for $\lambda^{1}, \lambda^{2}$ and $\lambda^{3}$, i.e., for $\lambda \geq 0$ the level sets are cross-shaped w.r.t. 0 , the unique continuous optimal solution in these cases. Whereas for $\lambda^{4}$ the level set is not crossshaped with respect to any point. Also $g_{\lambda^{4}}\left(x^{f}\right)$ does not have the Rounding Property: $\bar{X}=$ $\left\{\left(x_{1}, x_{2}\right): x_{1}^{2}=\left|x_{2}\right|\right\}$ and $X^{*}=\bar{X} \cap \mathbb{Z}^{2}=\left\{\left(x_{1}, x_{2}\right): x_{1} \in \mathbb{Z},\left|x_{2}\right|=x_{1}^{2}\right\}$. This means, e.g. $(108.16,10.4) \in \bar{X}$ but $\operatorname{Round}((108.16,10.4))=\{(108,10),(109,10),(108,11),(109,11)\}$, i.e., $\operatorname{Round}((108.16,10.4)) \cap X^{*}=\emptyset$.

If we assume $f_{i}(x)=x$ for all $i=1, \ldots, n$ we get that $g_{\lambda}(x)$ is convex if $\lambda_{1} \leq \lambda_{2} \leq \ldots \leq \lambda_{n}$ (even "if and only if", compare Grzybowski et al. [2011]). But we see in the example in Figure 3.14 that the level sets do not have to be cross-shaped if $\lambda_{1}<0$.

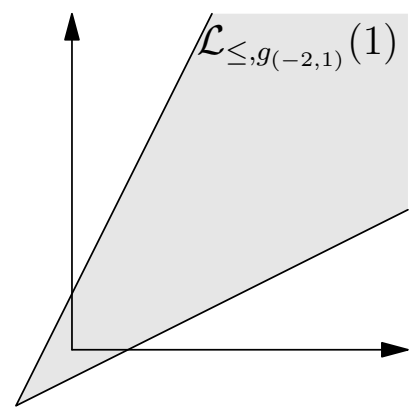

Figure 3.14.: The ordered median function $g_{\lambda}(x)$ for increasing $\lambda$ need not have cross-shaped level sets.

This raises the question: are the level sets of $g_{\lambda}\left(x^{f}\right)$ cross-shaped only if $\lambda_{i} \geq 0$ for all $i$ ?

The answer is "no": compare the example $f_{1}(x)=x^{2}$ and $f_{2}(x)=0$. Let furthermore $\lambda=(-1,1)$. Then we have $f\left(x_{1}, x_{2}\right)=x_{1}^{2}$, i.e., $\bar{X}=\left\{\left(0, x_{2}\right): x_{2} \in \mathbb{R}\right\}$ and the level sets are of the form $\mathcal{L}_{\leq}(z)=\left\{\left(x_{1}, x_{2}\right): x_{1}^{2} \leq z\right\}$ and hence cross-shaped w.r.t. every $\bar{x} \in \bar{X}$.

Remark 3.25. In general we do not get the Strong Rounding Property in Lemma 3.23 since not even a convex function in one dimension need have the Strong Rounding Property. 
The special case of minimizing separable convex functions over the integer lattice has already been considered in the literature. We mention the work of Hochbaum and Shanthikumar [1990] who consider the following problem

$$
\begin{array}{ll}
(I P) \min & f(x) \\
\text { s.t. } & A x \geq b \\
& x \in \mathbb{Z}^{n}
\end{array}
$$

where $f: \mathbb{R}^{n} \rightarrow \mathbb{R}$ is a separable convex function and give the following proximity result: for each optimal solution $\bar{x}$ to $(C P)$, there exists an optimal solution $x^{*}$ for $(I P)$ such that $\left\|\bar{x}-x^{*}\right\|_{\infty} \leq n \cdot \Delta$, where $\Delta$ is the maximum of the absolute values of the determinants of the square submatrices of $A$.

For the special case of $A=\left(\begin{array}{c}I_{n} \\ -I_{n}\end{array}\right)$ and $b=\left(\begin{array}{c}l \\ -u\end{array}\right)$ we get

$$
\begin{array}{lll}
(I P) & \min & f(x) \\
\text { s.t. } & l \leq x \leq u \\
& x \in \mathbb{Z}^{n}
\end{array}
$$

where $f: \mathbb{R}^{n} \rightarrow \mathbb{R}$ is a separable convex function and $l \leq u, l, u \in \mathbb{R}^{n}$.

Let $\bar{x}$ be any optimal solution to $(C P)$. Lemma 3.21 shows that the level sets $\mathcal{L}_{\leq}(z)$ are cross-shaped w.r.t $\bar{x}$ for all $z \geq f(\bar{x})$. The feasible set $F=\left\{x \in \mathbb{R}^{n}: l \leq x \leq u\right\}$ is a box and therefore cross-shaped w.r.t. every point $x \in F$ (compare Lemma 3.5). As $\bar{x}$ is an optimal solution to $(C P)$ we get $\bar{x} \in F$ and thus $\mathcal{L}_{\leq}(z)$ and $F$ are both cross-shaped w.r.t. $\bar{x}$. It follows that $\mathcal{L}_{\leq}^{F}(z)$ is also cross-shaped w.r.t. $\bar{x}$ for all $z \geq f(\bar{x})$, hence $(f, F)$ has the rounding property due to Theorem 3.6.

The proximity result in Hochbaum and Shanthikumar [1990] would give us $\left\|\bar{x}-x^{*}\right\|_{\infty} \leq n$ (since $\Delta=1$ ) whereas the Rounding Property gives us the stronger bound $\left\|\bar{x}-x^{*}\right\|_{\infty} \leq 1$ as we know that there is an $x^{*} \in \operatorname{Round}(\bar{x})$.

This means that in this special case we get a stronger proximity result than Hochbaum and Shanthikumar [1990] but we are limited to the case of box constraints.

While the result in Hochbaum and Shanthikumar [1990] holds for more complicated constraints our result holds for more complicated objective functions than just separable convex ones: the property of having cross-shaped level sets is by far not limited to separable quasiconvex functions, compare for example the function $f(x)=\|x\|_{\infty}$ which has cross-shaped level sets w.r.t. 0 but is not separable:

Assume that $f\left(x_{1}, x_{2}\right)=\max \left\{\left|x_{1}\right|,\left|x_{2}\right|\right\}$ is separable, i.e., there are $f_{1}: \mathbb{R} \rightarrow \mathbb{R}$ and $f_{2}: \mathbb{R} \rightarrow \mathbb{R}$, s.t. $f\left(x_{1}, x_{2}\right)=f_{1}\left(x_{1}\right)+f_{2}\left(x_{2}\right)$. Let $|y| \leq 1$, then we get

$$
f_{1}(y)+f_{2}(1)=f(y, 1)=\|(y, 1)\|_{\infty}=1=\|(1, y)\|_{\infty}=f(1, y)=f_{1}(1)+f_{2}(y) .
$$

Together this means, $\|(y, y)\|_{\infty}=f(y, y)=f_{1}(y)+f_{2}(y)=\underbrace{2-f_{1}(1)-f_{2}(1)}_{=\text {const }}$ for all $|y| \leq 1$ which is obviously a contradiction. 


\subsubsection{Norms}

We have already shown that in general functions of the form $f: \mathbb{R}^{n} \rightarrow \mathbb{R}, x \mapsto\|x-a\|$ for some $a \in \mathbb{R}^{n}$ and a norm $\|\cdot\|: \mathbb{R}^{n} \rightarrow \mathbb{R}$ do not have the Rounding Property, compare again Figure 1.13. Nevertheless, there are some special cases of norms, like the already mentioned $p$-norms, for which $f(x)=\|x-a\|$ has the Rounding Property or even the Strong Rounding Property. In this section we investigate for some special cases of norms whether they have the (Strong) Rounding Property or not.

We use in this section that $\|x\|=\lambda$ if and only if $x \in \lambda \cdot B_{\|\cdot\|}$ and $x \notin \mu \cdot B_{\|\cdot\|}$ for all $\mu<\lambda$ where $B_{\|\cdot\|}:=\left\{x \in \mathbb{R}^{n}:\|x\| \leq 1\right\}$ is the unit ball of the norm $\|\cdot\|$. This means that the level sets of $f(x):=\|x-a\|$ are of the form $\mathcal{L}_{\leq}(z)=a+z \cdot B_{\|\cdot\|}$ for all $z>0$.

We start with absolute norms.

\section{Absolute Norms}

Definition 3.26 (Bauer et al. [1961]). A norm $\|\cdot\|$ is called absolute if $\|x\|=\||x|\|$ for all $x \in \mathbb{R}^{n}$.

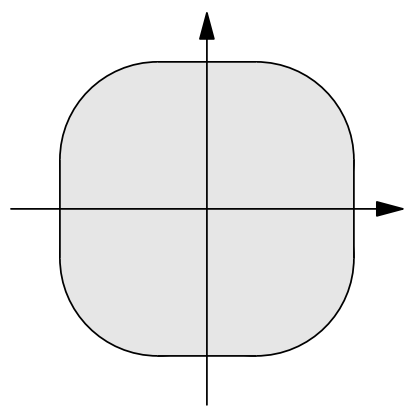

Figure 3.15.: An example for a unit ball of an absolute norm.

Lemma 3.27. $B_{\|\cdot\|}:=\left\{x \in \mathbb{R}^{n}:\|x\| \leq 1\right\}$ is cross-shaped and coordinate axially symmetric w.r.t. 0 if and only if $\|\cdot\|$ is absolute. This means that $\left(\|x-a\|, \mathbb{R}^{n}\right)$ for some $a \in \mathbb{R}^{n}$ has the Strong Rounding Property if $\|\cdot\|$ is absolute.

Proof. " $\Rightarrow$ ": let $u \in \mathbb{R}^{n},\|u\|=\lambda \Leftrightarrow u \in \mathcal{L}_{\leq}(\lambda)=\lambda \cdot B_{\|\cdot\|}$ and $u \notin \mathcal{L}_{\leq}(\mu)=\mu \cdot B_{\|\cdot\|}$ for all $\mu<\lambda$. Since $\lambda \cdot B_{\|\cdot\|}$ is coordinate axially symmetric w.r.t. 0 also $|u| \in \lambda \cdot B_{\|\cdot\| \cdot}$. Assume that $|u| \in \mu \cdot B_{\|\cdot\|}$ for $\mu<\lambda$ then we would also get $u \in \mu \cdot B_{\|\cdot\|}$ by the same argument. Therefore $\||u|\|=\lambda=\|u\|$. Hence $\|\cdot\|$ is absolute.

$" \Leftarrow$ ": $B_{\|\cdot\|}$ is also convex since it is the unit ball of a norm, Lemma 3.13 gives us that it is coordinate axially symmetric w.r.t. 0 and using Corollary 3.19 we get that it is also cross-shaped. 
In Bauer et al. [1961] it is shown that every absolute norm is monotonic and vice versa where a norm is called monotonic if $\left|x_{i}\right| \leq\left|y_{i}\right|$ for all $i=1, \ldots, n$ implies $\|x\| \leq\|y\|$ for all $x, y \in \mathbb{R}^{n}$. This means that the result of Lemma 3.27 also holds for monotonic norms. There are some more properties of norms that are equivalent to absoluteness (compare Johnson and Nylen [1991]) but we only state monotonicity here since it is the most common one.

A class of absolute norms are the $p$-norms for $p \in[1, \infty]$. But note that a similar result also holds for the class of functions $f_{p}(x):=\left(\sum_{i=1}^{n}\left|x_{i}-a_{i}\right|^{p}\right)^{1 / p}$ for $p \in(0,1)$ which are of course not norms.

Lemma 3.28. $\left(f_{p}^{a}(x)=\left(\sum_{i=1}^{n}\left|x_{i}-a_{i}\right|^{p}\right)^{1 / p}, \mathbb{R}^{n}\right)$ for $a \in \mathbb{R}^{n}$ and $p \in(0,1)$ has the Strong Rounding Property.

Proof. (i) $a$ is the unique continuous minimizer as $f_{p}^{a}(x)>0=f_{p}^{a}(a)$ for all $x \neq a$ and for all $p \in(0,1)$.

(ii) $\mathcal{L}_{\leq}(z)$ is cross-shaped w.r.t. $a$ for all $z \geq f_{p}^{a}(a)=0$ :

let $y \in \mathcal{L}_{\leq}(z)$, i.e., $f_{p}^{a}(y) \leq z$ and $x \in[a, y]_{1}$, i.e., $x_{i}=\lambda_{i} a_{i}+\left(1-\lambda_{i}\right) y_{i}$ for all $i=1, \ldots, n$ where $\lambda_{i} \in[0,1]$. This means,

$$
f(x)=\left(\sum_{i=1}^{n}\left|\left(1-\lambda_{i}\right)\left(y_{i}-a_{i}\right)\right|^{p}\right)^{1 / p} \leq\left(\sum_{i=1}^{n}\left|y_{i}-a_{i}\right|^{p}\right)^{1 / p}=f_{p}^{a}(y) \leq z .
$$

This means that $[a, y]_{1} \subseteq \mathcal{L}_{\leq}(z)$ for all $y \in \mathcal{L}_{\leq}(z)$ and all $z \geq 0$.

(iii) $\mathcal{L}_{\leq}(z)$ is coordinate axially symmetric w.r.t. $a$ for all $z \geq f_{p}^{a}(a)=0$ due to Lemma 3.13.

Example 3.29. We consider the function $f_{p}^{a}$ for $a=(0.2,0.7)$ and $p=\frac{1}{2}$ and depict the level set $\mathcal{L}_{\leq}\left(f_{\frac{1}{2}}^{a}\left(x^{*}\right)\right)$, compare Figure 3.16.

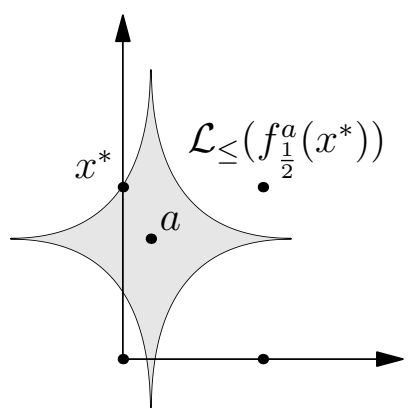

Figure 3.16.: An example for the family of functions considered in Lemma 3.28.

Next we investigate block norms. 


\section{Block norms}

Definition 3.30 (Ward and Wendell [1985]). A norm $\|\cdot\|$ with a polyhedral unit ball in $\mathbb{R}^{n}$ is called a block norm.

Block norms do in general not have the Rounding Property, compare again the example in Figure 1.13.

But there is a special class of block norms that actually has the Rounding Property.

Definition 3.31 (Ward and Wendell [1980]). Let $\alpha_{1}, \alpha_{2} \in \mathbb{R}_{0}^{+},\left(\alpha_{1}, \alpha_{2}\right) \neq(0,0)$. Then the weighted one-infinity norm is given as

$$
\|x\|_{\left(\alpha_{1}, \alpha_{2}\right)}:=\alpha_{1}\|x\|_{1}+\alpha_{2} \sqrt{2}\|x\|_{\infty} .
$$

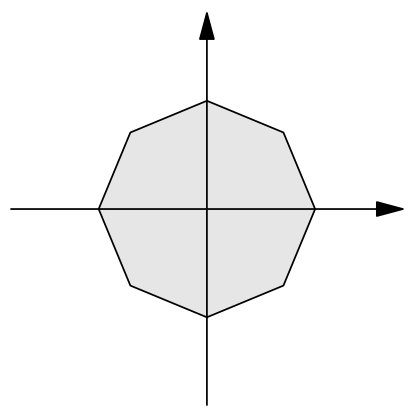

Figure 3.17.: An example for a unit ball of a one-infinity norm.

Lemma 3.32. $\left(\|x-a\|_{\left(\alpha_{1}, \alpha_{2}\right)}, \mathbb{R}^{n}\right)$ for $a \in \mathbb{R}^{n}$ and $\alpha_{1}, \alpha_{2} \in \mathbb{R}_{0}^{+},\left(\alpha_{1}, \alpha_{2}\right) \neq(0,0)$ has the Strong Rounding Property.

Proof. Since both $\|\cdot\|_{\infty}$ and $\|\cdot\|_{1}$ are absolute, so is $\|\cdot\|_{\left(\alpha_{1}, \alpha_{2}\right)}$. Therefore the result follows from Lemma 3.27.

The cornered p-norm as presented in Büsing et al. [2011]:

$$
\begin{gathered}
\|\| x \mid\left\|_{p}:=\right\| x\left\|_{\infty}+\frac{1}{p}\right\| x \|_{1} \quad \text { for } p \in[1, \infty), \\
\|\| x\|\|_{\infty}:=\|x\|_{\infty}
\end{gathered}
$$

is a special case of a one-infinity-norm and therefore Lemma 3.32 also holds for cornered $p$-norms.

The next class of norms we analyze is the one of weighted norms. 


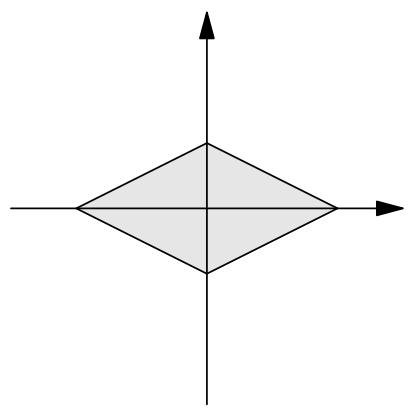

Figure 3.18.: An example for a unit ball of a weighted 1-norm.

\section{Weighted norms}

Definition 3.33 (similar to Büsing et al. [2011]). For any norm $\|\cdot\|$ on $\mathbb{R}^{n}$ and any $\lambda \in \mathbb{R}^{n}$ with $\lambda>0$ we define the weighted version of $\|\cdot\|$

$$
\|x\|^{\lambda}:=\left\|\left(\lambda_{1} x_{1}, \ldots, \lambda_{n} x_{n}\right)\right\| .
$$

Lemma 3.34. If $B_{\|\cdot\|}$ is cross-shaped w.r.t. 0 for some norm $\|\cdot\|$ so is $B_{\|\cdot\| \lambda}$ for its weighted version for any $\lambda \in \mathbb{R}^{n}$ with $\lambda>0$. If $B_{\|\cdot\|}$ is coordinate axially symmetric w.r.t. 0 so is $B_{\|\cdot\| \lambda}$. This means that $\left(\|x-a\|^{\lambda}, \mathbb{R}^{n}\right)$ for some $a \in \mathbb{R}^{n}$ has the (Strong) Rounding Property.

Proof. If $B_{\|\cdot\|}$ is cross-shaped w.r.t. 0 we know that if $\|x\| \leq 1$ also $\left\|\left(\mu_{1} x_{1}, \ldots, \mu_{n} x_{n}\right)\right\| \leq 1$ if $\mu_{i} \in[0,1]$ for all $i=1, \ldots, n$. Let now $\lambda \in \mathbb{R}^{n}, \lambda>0$ and let $x \in B_{\|\cdot\|^{\lambda}}$, i.e., $\|x\|^{\lambda}=$ $\left\|\left(\lambda_{1} x_{1}, \ldots, \lambda_{n} x_{n}\right)\right\| \leq 1$. Let $y \in[0, x]_{1}$, i.e., $y_{i}=\mu_{i} x_{i}$ for all $i=1, \ldots, n$. Then we have

$$
\|y\|^{\lambda}=\left\|\left(\lambda_{1} y_{1}, \ldots, \lambda_{n} y_{n}\right)\right\|=\left\|\left(\mu_{1} \lambda_{1} x_{1}, \ldots, \mu_{n} \lambda_{n} x_{n}\right)\right\| \leq 1
$$

since $\left\|\left(\lambda_{1} x_{1}, \ldots, \lambda_{n} x_{n}\right)\right\| \leq 1$ and $B_{\|\cdot\|}$ cross-shaped w.r.t. 0 .

If $B_{\|\cdot\|}$ is coordinate axially symmetric w.r.t. 0 we know that if $\|x\| \leq 1$ also $\left\|\left(x_{1}, \ldots, x_{i-1},-x_{i}, x_{i+1}, \ldots, x_{n}\right)\right\| \leq 1$ for all $i \in\{1, \ldots, n\}$. Let now $\lambda \in \mathbb{R}^{n}, \lambda>0$ and let $x \in B_{\|\cdot\|^{\lambda}}$, i.e., $\|x\|^{\lambda}=\left\|\left(\lambda_{1} x_{1}, \ldots, \lambda_{n} x_{n}\right)\right\| \leq 1$. Define $z_{j}^{i}:=\left\{\begin{array}{ll}x_{j} & \text { if } j \neq i \\ -x_{i} & \text { otherwise }\end{array}\right.$. Then we have $\left\|z^{i}\right\|^{\lambda}=\left\|\left(\lambda_{1} x_{1}, \ldots, \lambda_{i-1} x_{i-1},-\lambda_{i} x_{i}, \lambda_{i+1} x_{i+1}, \ldots, \lambda_{n} x_{n}\right)\right\| \leq 1$ for all $i \in\{1, \ldots, n\}$ since $\|x\|^{\lambda} \leq 1$. And hence $B_{\|\cdot\|^{\lambda}}$ is coordinate axially symmetric w.r.t. 0 .

We end with a short note on elliptic norms.

\section{Elliptic norms}

Definition 3.35 (e.g. Körner [2011]). A norm $\|\cdot\|$ is called elliptic if its unit ball is an ellipsoid. ${ }^{1}$ 


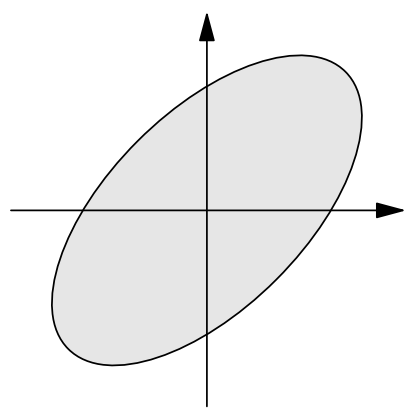

Figure 3.19.: An example for a unit ball of an elliptic norm.

Chapter 5 is dedicated to the special case of convex quadratic functions which includes the function $f(x)=\|x-a\|$ for some $a \in \mathbb{R}^{n}$ and an elliptic norm $\|\cdot\|$. For the sake of completeness we cite the most important results here.

(i) In general, an elliptic norm need not have the Rounding Property, compare the counterexample in Figure 5.1 where the depicted set could be the unit ball of an elliptic norm.

(ii) An ellipsoid is cross-shaped if and only if it is axis-parallel. In this case it is also coordinate axially symmetric (Lemma 5.4).

(iii) An ellipsoid $\left\{x \in \mathbb{R}^{n}: x^{t} Q x \leq z\right\}$ (for $Q$ positive definite) is $\alpha$-quasi-round w.r.t. 0 if and only if $\frac{1}{\sqrt{\lambda_{\min }}}-\frac{1}{\sqrt{\lambda_{\max }}} \leq \alpha$, where $\lambda_{\min }$ and $\lambda_{\max }$ are the smallest and largest eigenvalues of $Q$ (Lemma 5.8).

\subsubsection{Location theory}

We end this section by giving some applications to location theory. Problems from location theory are an interesting field of application for the Rounding Property. First of all typically the dimension $n$ is rather small, which means that comparing the up to $2^{n}$ points in $\operatorname{Round}(\bar{x}) \cap$ $F$ is practicable and secondly the continuous counterparts are well-researched and for many problems an efficient algorithm is at hand.

In the following we investigate two different types of location problems: given existing facilities $p^{1}, \ldots, p^{M} \in \mathbb{R}^{n}$, positive weights $w_{m}$ for all $m=1, \ldots, M$ and a norm $\|\cdot\|: \mathbb{R}^{n} \rightarrow \mathbb{R}$, we consider the integer median location problem

$$
\begin{array}{ll}
\min & \sum_{m=1}^{M} w_{m}\left\|x-p^{m}\right\| \\
\text { s.t. } & x \in \mathbb{Z}^{n}
\end{array}
$$

and the integer center location problem

$$
\begin{array}{ll}
\min & \max _{m=1}^{M} w_{m}\left\|x-p^{m}\right\| \\
\text { s.t. } & x \in \mathbb{Z}^{n} .
\end{array}
$$

\footnotetext{
${ }^{1}$ In geometry these norms are often also called Euclidean (e.g. Thompson [1996]).
} 
A survey on continuous median location problems can be found in Drezner et al. [2002] and an overview on continuous center location problems is provided by Plastria [2002].

Integer location problems arise for example in average sampling approaches where an integer representative for a set of sampled points is to be found. Finding the best integer point to represent a set of sampled points can be modeled as an integer location problem. An other application are location problems on a grid -for example a crossing has to be chosen in a city with only orthogonal and equidistant streets.

We start by investigating median location problems and observe first of all that in general Problem 3.1 does not have the Rounding Property, consider the following example.

Example 3.36. Given four points $p^{1}=(0,0), p^{2}=(0,1), p^{3}=(3,0)$ and $p^{4}=(2.05,0.3)$ with equal weights $w_{1}=\ldots=w_{4}$ in the plane, we want to solve the following median problem

$$
\min \left\{\left\|x-p^{1}\right\|_{2}+\left\|x-p^{2}\right\|_{2}+\left\|x-p^{3}\right\|_{2}+\left\|x-p^{4}\right\|_{2}: x \in \mathbb{Z}^{2}\right\} .
$$

An optimal solution to its continuous relaxation is $\bar{x}=p^{4}$ (compare for example Drezner et al. [2002]) and some of the first level sets are depicted in Figure 3.20.

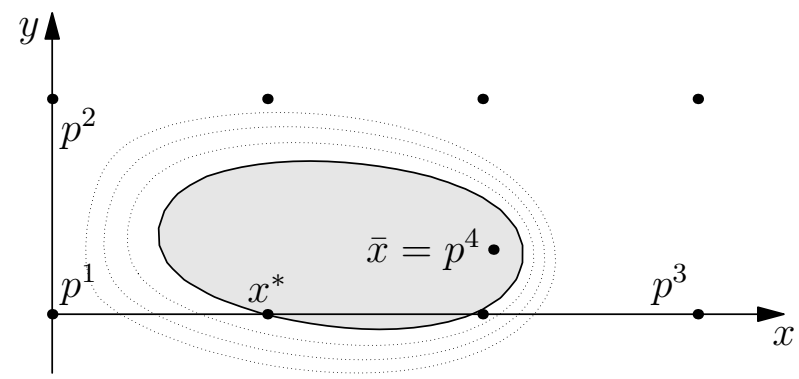

Figure 3.20.: The median problem with Euclidean norm does in general not have the Rounding Property.

We see that the unique optimal solution to the integer median problem is $x^{*}=(1,0)$ and hence $\operatorname{Round}(\bar{x}) \cap X^{*}=\emptyset$ and the problem does not have the Rounding Property.

Note that this example also shows why it is important to assume in Corollary 3.8 and Corollary 3.12 (vii) that the set of continuous minimizers to $f_{1}$ and $f_{2}$ coincide, to make sure that the level sets of $f_{1}+f_{2}$ are cross-shaped: obviously the functions $f_{i}=\left\|x-p_{i}\right\|_{2}$ have cross-shaped and even coordinate axially symmetric level sets, but since the sets of continuous minimizers do not coincide we do not get the Rounding Property here.

Next we note that for the special case of the 1-norm problem (3.1) has the Rounding Property since the objective function is separable convex, compare Lemma 3.21. Furthermore, the continuous relaxation of this problem can be solved in $\mathcal{O}(n M)$ time by calculating the coordinate wise median (compare Drezner et al. [2002]). This means, in fixed dimension problem (3.1) can be solved in $\mathcal{O}\left(n M 2^{n}\right)$ time. An example of a median location problem with 1-norm is given next.

Example 3.37. Given three points $p^{1}=(1,1), p^{2}=(2,3)$ and $p^{3}=(4,1.5)$ with equal weights $w_{1}=w_{2}=w_{3}$ in the plane, we want to solve the following median problem

$$
\min \left\{\left\|x-p^{1}\right\|_{1}+\left\|x-p^{2}\right\|_{1}+\left\|x-p^{3}\right\|_{1}: x \in \mathbb{Z}^{2}\right\} .
$$


Then the unique continuous optimal solution is $\bar{x}=(2,1.5)$ and the level sets can be seen in Figure 3.21 (compare for example Hamacher and Nickel [1995]).

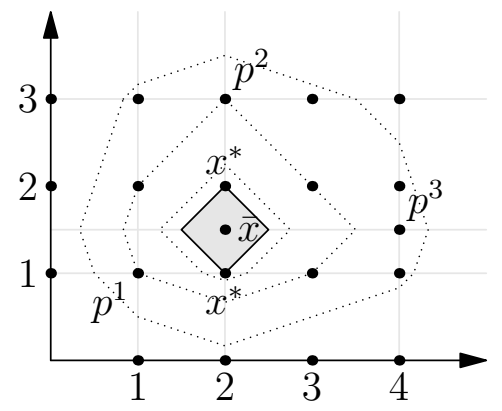

Figure 3.21.: The median problem with 1-norm has cross-shaped level sets and hence the Rounding Property.

Now we look at the $\infty$-norm and observe that the median problem with $\infty$-norm does in general not have the Rounding Property: consider the following example.

Example 3.38. Given two points $p^{1}=(0.8,0.6)$ and $p^{2}=(3,1)$ with equal weights $w_{1}=w_{2}$, we want so solve the following median problem:

$$
\min \left\{\left\|x-p^{1}\right\|_{\infty}+\left\|x-p^{2}\right\|_{\infty}: x \in \mathbb{Z}^{2}\right\} .
$$

The set of optimal solutions to the continuous relaxation is given by $\bar{X}=\left\{\left(x_{1}, x_{2}\right) \in \mathbb{R}^{2}\right.$ : $1.4-$ $\left.x_{1} \leq x_{2} \leq 4-x_{1}, x_{1}-2 \leq x_{2} \leq x_{1}-0.2\right\}$ (compare e.g. Hamacher and Nickel [1995]). Hence $X^{*}=\{(2,0),(2,1),(3,1)\}$ and $\operatorname{Round}\left(p^{1}\right) \cap X^{*}=\emptyset$ even though $p^{1} \in \bar{X}$, compare also Figure 3.22.

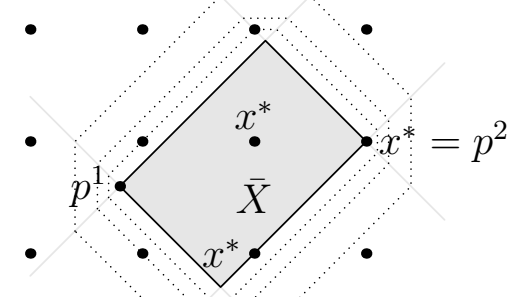

Figure 3.22.: The median problem with $\infty$-norm does in general not have the Rounding Property.

Another median problem with cross-shaped level sets is the the median problem with squared Euclidean norm:

$$
\begin{array}{ll}
\min & \sum_{m=1}^{M} w_{m}\left\|x-p^{m}\right\|_{2}^{2} \\
\text { s.t. } & x \in \mathbb{Z}^{n}
\end{array}
$$


which is strictly speaking not of form 3.1 since $\|\cdot\|_{2}^{2}$ is not a norm.

The level sets of this problem are Euclidean balls about the unique optimal solution to the continuous relaxation $\bar{x}=\frac{1}{\sum_{m=1}^{M} w_{m}}\left(\sum_{m=1}^{M} w_{m} p_{1}^{m}, \ldots, \sum_{m=1}^{M} w_{m} p_{n}^{m}\right)$ (compare Eyster and White [1973]) and hence cross-shaped and coordinate axially symmetric about $\bar{x}$. Therefore the problem even has the Strong Rounding Property.

Example 3.39. Given again the three points from Example $3.37 p^{1}=(1,1), p^{2}=(2,3)$ and $p^{3}=(4,1.5)$ with equal weights $w_{1}=w_{2}=w_{3}$ in the plane, we want to solve the following median problem

$$
\min \left\{\left\|x-p^{1}\right\|_{2}^{2}+\left\|x-p^{2}\right\|_{2}^{2}+\left\|x-p^{3}\right\|_{2}^{2}: x \in \mathbb{Z}^{2}\right\} .
$$

The unique continuous minimizer is

$$
\bar{x}=\frac{1}{3}(7,5.5)=\left(\frac{7}{3}, \frac{11}{6}\right)
$$

and the level sets are concentric circles as depicted in Figure 3.23. Hence the unique optimal solution is $x^{*}=\lfloor\bar{x}\rceil=(2,2)$.

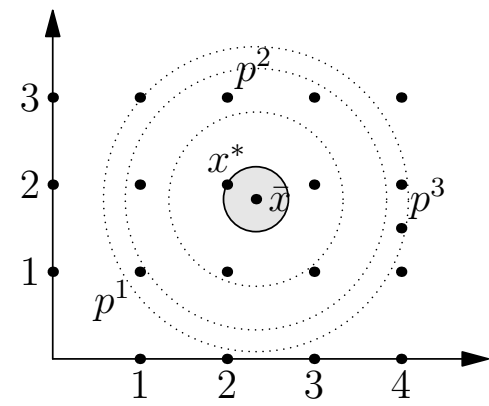

Figure 3.23.: The median problem with squared Euclidean norm has the Strong Rounding Property.

In general the problem

$$
\begin{array}{ll}
\min & \sum_{m=1}^{M} w_{m}\left\|x-p^{m}\right\|_{p}^{p} \\
\text { s.t. } & x \in \mathbb{Z}^{n}
\end{array}
$$

for any $p \in[1, \infty)$ has the Rounding Property since the objective function is separable convex. Now we move on to center location problems and observe first of all that the level sets of $f(x)=\max _{m=1}^{M} w_{m}\left\|x-p^{m}\right\|$ are the intersection of scaled and shifted unit balls (compare e.g. Hamacher and Schöbel [1997]):

$$
\mathcal{L}_{\leq, f}(z)=\bigcap_{m=1}^{M}\left(\frac{z}{w_{m}} \cdot B_{\|\cdot\|}+p^{m}\right) .
$$

Again we start with a negative result for the Euclidean norm: in general the center problem with Euclidean norm does not have the Rounding Property, compare the following example. 
Example 3.40. Given two points $p^{1}=\left(\frac{73}{78}, \frac{11}{39}\right)$ and $p^{2}=\left(\frac{473}{78},-\frac{89}{39}\right)$ with equal weights $w_{1}=$ $w_{2}$ in the plane, we want to solve the following center problem

$$
\min \left\{\max \left\{\left\|x-p^{1}\right\|_{2},\left\|x-p^{2}\right\|_{2}\right\}: x \in \mathbb{Z}^{2}\right\} .
$$

The unique optimal solution to its continuous relaxation is the intersection of the bisector with the connecting line $\bar{x}=(3.5,-1)$ (compare Figure 3.24). But the unique integer minimizer is $x^{*}=(4,0)$, compare also Figure 3.24.

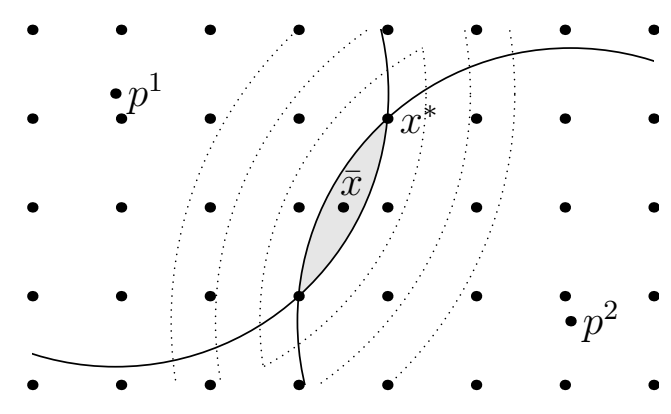

Figure 3.24.: The center problem with Euclidean norm does in general not have the Rounding Property.

We see that the problem does not have the Rounding Property.

Note that this is also a counterexample for the squared Euclidean norm case, since the level sets have the same geometric shape.

Turning our attention to the 1-norm we see that this center problem does not have the Rounding Property either, compare the following example.

Example 3.41. Given the two points $p^{1}=\left(\frac{7}{4}, \frac{1}{2}\right), p^{2}=\left(\frac{7}{8}-42, \frac{1}{2}\right)$ with weights $w_{1}=1$ and $w_{2}=\frac{1}{48}$ we want to solve the center problem with 1-norm

$$
\min \left\{\max \left\{\left\|x-p^{1}\right\|_{1},\left\|x-p^{2}\right\|_{1}\right\}: x \in \mathbb{Z}^{2}\right\} .
$$

We get the unique continuous optimal solution $\bar{x}=\left(\frac{7}{8}, \frac{1}{2}\right), X^{*}=\{(2,0),(2,1)\}$ and see that $\operatorname{Round}(\bar{x}) \cap X^{*}=\emptyset$, compare also Figure 3.25.

On the other hand, now the $\infty$-norm leads to the Rounding Property since the intersection of finitely many scaled and shifted $\infty$-norm unit balls are boxes and hence cross-shaped w.r.t. any continuous optimal solution. Compare also the following example.

Example 3.42. Given three points $p^{1}=(1.2,1), p^{2}=(0,0.8)$ and $p^{3}=(1.6,2.2)$ with weights $w_{1}=1, w_{2}=\frac{1}{2}$ and $w_{3}=\frac{1}{3}$ in the plane, we want to solve the following center problem

$$
\min \left\{\max \left\{\left\|x-p^{1}\right\|_{\infty},\left\|x-p^{2}\right\|_{\infty},\left\|x-p^{3}\right\|_{\infty}\right\}: x \in \mathbb{Z}^{2}\right\} .
$$

The set of continuous minimizers is $\bar{X}=\left\{\left(x_{1}, x_{2}\right): x_{1}=0.8,1 \leq x_{2} \leq 1.4\right\}$, the level sets are boxes as depicted in Figure 3.26 and the unique integer minimizer is $x^{*}=(1,1)$. This means, this example even has the Strong Rounding Property. 


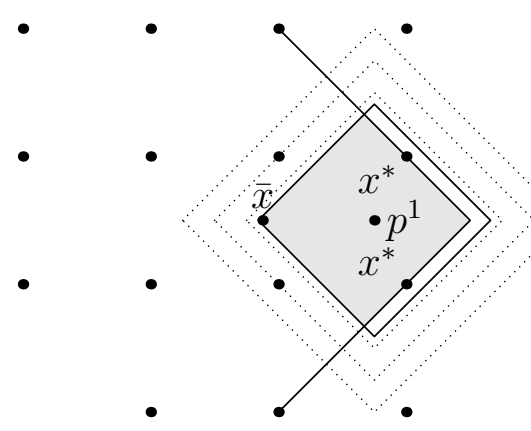

Figure 3.25.: The center problem with 1-norm does in general not have the Rounding Property.

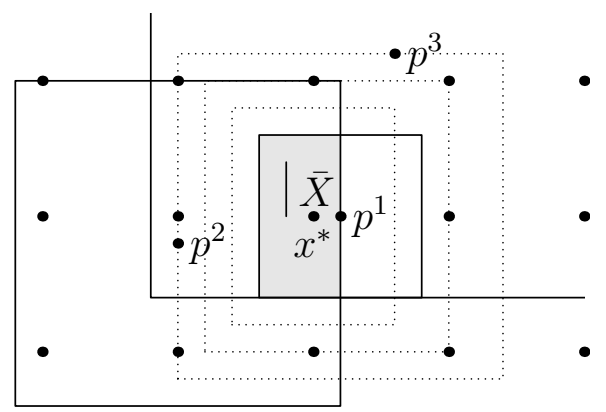

Figure 3.26.: The center problem with $\infty$-norm has the Rounding Property.

Summarizing the results of this section we get the following table.

\begin{tabular}{c|c|c} 
distance & problem & Rounding Property \\
\hline \hline$\|\cdot\|_{2}$ & median & no \\
$\|\cdot\|_{2}$ & center & no \\
$\|\cdot\|_{1}$ & median & Rounding Property \\
$\|\cdot\|_{1}$ & center & no \\
$\|\cdot\|_{\infty}$ & median & no \\
$\|\cdot\|_{\infty}$ & center & Rounding Property \\
$\|\cdot\|_{2}^{2}$ & median & Strong Rounding Property \\
$\|\cdot\|_{2}^{2}$ & center & no
\end{tabular}




\subsection{Quasi-cross-shaped sets}

Just as we defined quasi-round sets to be a generalization of round sets we do the same for cross-shaped sets. This means, we enclose a given set between two scaled versions of a cross-shaped set where the difference between the scaling factors is limited. For general (not necessarily cross-shaped) sets we call this property $\epsilon$-similarity. In the following we investigate under which assumptions quasi-cross-shaped sets guarantee the Rounding Property.

Definition 3.43. Given $\epsilon \geq 0$ we call a set $M \subseteq \mathbb{R}^{n} \epsilon$-similar to a set $A \subseteq \mathbb{R}^{n}$ w.r.t. $x^{0}$ if there are $\lambda_{1}, \lambda_{2} \in \mathbb{R}_{0}^{+}$such that

$$
\lambda_{1}\left(A-x^{0}\right)+x^{0} \subseteq M \subseteq \lambda_{2}\left(A-x^{0}\right)+x^{0}
$$

and $\lambda_{2}-\lambda_{1} \leq \epsilon$ and we call $M \epsilon$-quasi-cross-shaped w.r.t. $x^{0}$ if there exists a cross-shaped set $B \subseteq \mathbb{R}^{n}$ such that $M$ is $\epsilon$-similar to $B$ w.r.t. $x^{0}$.

Note if $A=B_{p}\left(x^{0}, 1\right)$ and $\epsilon=\alpha$ we get the definition of $(\alpha, p)$-quasi-round as in Definition 2.1 since $B_{p}\left(x^{0}, R\right)=R\left(B_{p}\left(x^{0}, 1\right)-x^{0}\right)+x^{0}$ and this also means that any $\alpha$-quasi-round set is also $\alpha$-quasi-cross-shaped.

Notation 3.44. Given a set $A \subseteq \mathbb{R}^{n}$, a point $x^{0} \in A$ and a point $x \in \mathbb{R}^{n}$ we use the following notation

$$
p_{A, x^{0}}(x):=\inf \left\{\lambda \geq 0: x \in \lambda\left(A-x^{0}\right)+x^{0}\right\} .
$$

If $A \subseteq \mathbb{R}^{n}$ is cross-shaped w.r.t. $x^{0},\left(p_{A, x^{0}}, \mathbb{R}^{n}\right)$ has the Rounding Property, since the level sets $\mathcal{L}_{\leq, p_{A, x^{0}}}(z)=z \cdot\left(A-x^{0}\right)+x^{0}$ are cross-shaped w.r.t. $x^{0}$ (compare Lemma 3.4).

Next we show that $\epsilon$-quasi-cross-shapedness guarantees the Rounding Property.

Theorem 3.45 (Rounding Property for quasi-cross-shaped sets). Let $(f, F)$ be given. Assume that for any optimal solution $\bar{x}$ to $(C P)$ and for any level $f(\bar{x}) \leq z \leq \min \{f(x): x \in$ $\operatorname{Round}(\bar{x}) \cap F\}$ the level set $\mathcal{L}_{\leq}^{F}(z)$ is $\epsilon(A, \bar{x}, F)$-quasi-cross-shaped w.r.t. $\bar{x}$ where

$$
\epsilon(A, \bar{x}, F):=\min _{x \in\left(\mathbb{Z}^{n} \cap F\right) \backslash \operatorname{Round}(\bar{x})} p_{A, \bar{x}}(x)-\min _{x \in \mathbb{Z}^{n} \cap F} p_{A, \bar{x}}(x) .
$$

Then $(f, F)$ has the Rounding Property.

By saying that a set $M$ is $\epsilon(A)$-quasi-cross-shaped we mean that there exists a cross-shaped set $A$ such that $M$ is $\epsilon(A)$-similar to $A$. Furthermore, we define $\infty-\infty=0$ here and note that $\epsilon(A, \bar{x}, F) \geq 0$ and hence $\epsilon(A, \bar{x}, F)$-similarity is well-defined.

Proof. Let $\bar{x} \in \bar{X}$ and $x \in \mathbb{Z}^{n} \cap F$. Using Lemma 1.18 we show that $\mathcal{L}_{\leq}^{F}(f(x)) \cap \operatorname{Round}(\bar{x}) \neq \emptyset$. If $\min \{f(u): u \in \operatorname{Round}(\bar{x}) \cap F\} \leq f(x)$ we are done. Otherwise we have

(i) $x \notin \operatorname{Round}(\bar{x})$ as $x \in F$ and

(ii) there exists a set $A$ that is cross-shaped w.r.t. $\bar{x}$ such that $\mathcal{L}_{\leq}^{F}(f(x))$ is $\epsilon(A, \bar{x}, F)$-similar to $A$ w.r.t. $\bar{x}$, i.e., there exist $\lambda_{1}, \lambda_{2} \in \mathbb{R}_{0}^{+}$s.t.

$$
\lambda_{1}(A-\bar{x})+\bar{x} \subseteq \mathcal{L}_{\leq}^{F}(f(x)) \subseteq \lambda_{2}(A-\bar{x})+\bar{x}
$$

and $\lambda_{2}-\lambda_{1} \leq \epsilon(A, \bar{x}, F)$. 
As $x \in \mathcal{L}_{\leq}^{F}(f(x))$ we have

$$
p_{A, \bar{x}}(x):=\inf \{\lambda \geq 0: x \in \lambda(A-\bar{x})+\bar{x}\} \leq \lambda_{2} .
$$

Let $\hat{x} \in \underset{\operatorname{argmin}}{\arg } p_{A, \bar{x}}(y) \cap \operatorname{Round}(\bar{x})$. Then we have $\min _{y \in \mathbb{Z}^{n} \cap F} p_{A, \bar{x}}(y) \geq p_{A, \bar{x}}(\hat{x})$ and hence $y \in \mathbb{Z}^{n}$

$\neq \emptyset$ as $\left(p_{A, \bar{x}}, \mathbb{R}^{n}\right)$ has the RP

$\epsilon(A, \bar{x}, F)=\min _{y \in\left(\mathbb{Z}^{n} \cap F\right) \backslash \operatorname{Round}(\bar{x})} p_{A, \bar{x}}(y)-\min _{y \in \mathbb{Z}^{n} \cap F} p_{A, \bar{x}}(y) \leq \min _{y \in\left(\mathbb{Z}^{n} \cap F\right) \backslash \operatorname{Round}(\bar{x})} p_{A, \bar{x}}(y)-p_{A, \bar{x}}(\hat{x})$.

This means,

$$
\begin{aligned}
p_{A, \bar{x}}(\hat{x}) & \leq \min _{y \in\left(\mathbb{Z}^{n} \cap F\right) \backslash \operatorname{Round}(\bar{x})} p_{A, \bar{x}}(y)-\epsilon(A, \bar{x}, F) \\
& \leq p_{A, \bar{x}}(x)-\epsilon(A, \bar{x}, F) \quad \text { as } x \in \mathbb{Z}^{n} \cap F, x \notin \operatorname{Round}(\bar{x}) \\
& \leq \lambda_{2}-\epsilon(A, \bar{x}, F) \leq \lambda_{1} .
\end{aligned}
$$

And therefore $\hat{x} \in \lambda_{1}(A-\bar{x})+\bar{x} \subseteq \mathcal{L}_{<}^{F}(f(x))$. Thus Round $(\bar{x}) \cap F \neq \emptyset$ and there exists $\widetilde{x} \in \operatorname{argmin}\{f(u): u \in \operatorname{Round}(\bar{x}) \cap F\}$. Then we have $f(\widetilde{x}) \leq f(\hat{x}) \leq f(x)<f(\widetilde{x})$. Thus the assumption $f(x)<\min \{f(u): x \in \operatorname{Round}(\bar{x}) \cap F\}$ leads to a contradiction.

Remark 3.46. (i) We note again that we are not looking for one $A$ that fits for all level sets and all $\bar{x} \in \bar{X}$ but for each level $f(\bar{x}) \leq z \leq \min \{f(x): x \in \operatorname{Round}(\bar{x}) \cap F\}$ and each $\bar{x} \in \bar{X}$ we can find $A(\bar{x}, z)$.

(ii) This theorem contains Theorem 3.6 as a special case.

(iii) If we consider the special case that $A-x^{0}$ is the unit ball of a norm $\|\cdot\|_{A}$. We get $x \in \lambda\left(A-x^{0}\right)+x^{0} \Leftrightarrow\left\|x-x^{0}\right\|_{A} \leq \lambda$ and hence $p_{A, x^{0}}=\left\|x-x^{0}\right\|_{A}$. This means,

$$
\epsilon(A, \bar{x}, F)=\min _{x \in\left(\mathbb{Z}^{n} \cap F\right) \backslash \operatorname{Round}(\bar{x})}\left\|x-x^{0}\right\|_{A}-\min _{x \in \mathbb{Z}^{n} \cap F}\left\|x-x^{0}\right\|_{A}
$$

and hence for example for $A=B_{p}\left(x^{0}, 1\right)$ we get $\epsilon(A, \bar{x}, F)=\alpha_{p}(\bar{x}, F)$. Therefore Theorem 3.45 also contains Theorem 2.5 as a special case: if the level set $\mathcal{L}_{\leq}^{F}(z)$ is $\alpha_{p}(\bar{x}, F)$ quasi-round w.r.t. $\bar{x}$ it is also $\alpha_{p}(\bar{x}, F)$-quasi-cross-shaped w.r.t. $\bar{x}$ as mentioned before.

(iv) The proof of Theorem 3.45 shows that it is not really necessary that $A$ is cross-shaped. We are going to relax that condition later, compare 4.2.

(v) The challenge is of course to decide whether there is a cross-shaped set $A$ such that $\mathcal{L}_{\leq}^{F}(z)$ is $\epsilon(A, \bar{x}, F)$-similar to $A$. This includes also the problem of determining $\epsilon(A, \bar{x}, F)$ for a given set $A$ which is not going to be easy (or even possible) in general. Also we point out that in a lot of cases $\epsilon(A, \bar{x}, F)$ is going to be 0 so that the assumption of this theorem is that the level set is cross-shaped.

(vi) In contrast to the quasi-round case it is not possible to shrink $\epsilon(A, \bar{x}, F)$ to even get the Strong Rounding Property, since even if the level sets are all cross-shaped we need not get the Strong Rounding Property.

We end this section by providing an example where Theorem 3.45 proves the Rounding Property and Theorems 3.6 and 2.5 do not. 
Example 3.47. In Figure 3.27 we see an example for a level set that is neither cross-shaped (compare $y$ ) nor $\left(\alpha_{p}, p\right)$-quasi-round for any $p$ (since $R-r>1$ and $\alpha_{p} \leq 1$ for all $p \in[1, \infty]$ ) but that has nevertheless the Rounding Property as can be shown by Theorem 3.45 with the set A depicted, since $A$ is cross-shaped and hence the level set is quasi-cross-shaped.

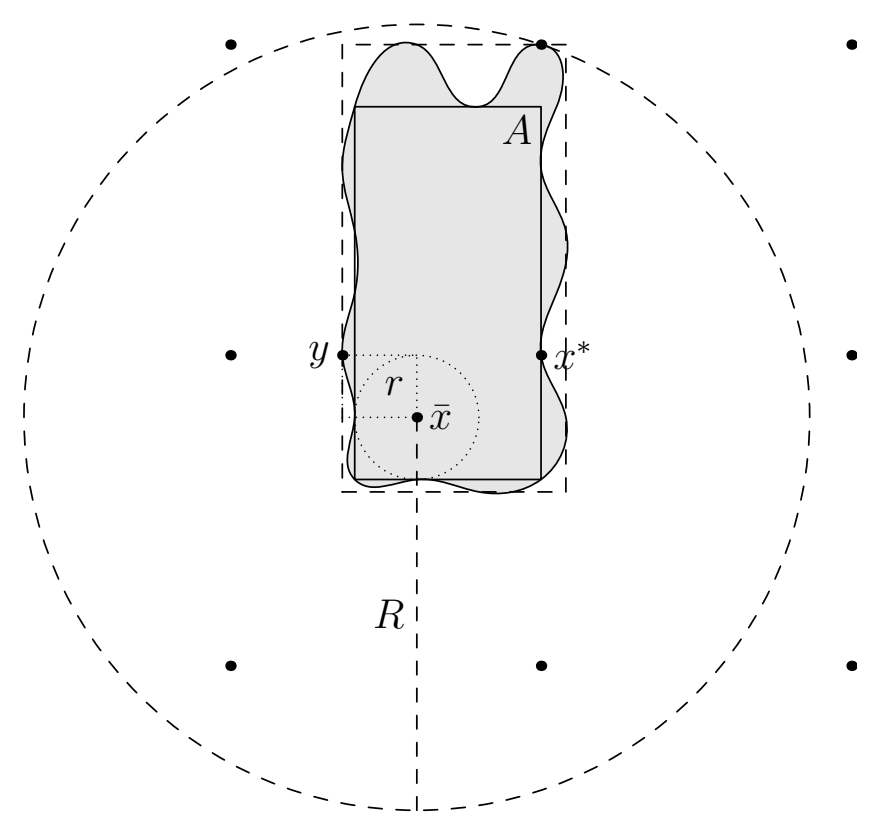

Figure 3.27.: An example for a level set whose Rounding Property can be shown by Theorem 3.45 but neither by Theorem 2.5 nor by Theorem 3.6. 


\subsection{Summary}

In this chapter we introduced the definition of a cross-shaped set: we say that a set $M \subseteq \mathbb{R}^{n}$ is cross-shaped with respect to a specified center $x^{0}$ if for any $y \in M$ also the 1-norm segment $\left[x^{0}, y\right]_{1}:=\left\{p \in \mathbb{R}^{:} p_{i}=\lambda_{i} x_{i}^{0}+\left(1-\lambda_{i}\right) y_{i}, \lambda_{i} \in[0,1] \forall i=1, \ldots, n\right\}$ is contained in $M$. We showed that the cross-shapedness of a set is preserved under certain set manipulations like scaling or translating and furthermore, that the only sets that are cross-shaped with respect to every point they contain, are boxes.

We are interested in cross-shaped sets since we showed in Theorem 3.6 that cross-shaped level sets induce the Rounding Property. The proof is, geometrically speaking, based on the observation that if $y$ is integer, the 1-norm segment $\left[x^{0}, y\right]_{1}$ contains at least one of the points of Round $\left(x^{0}\right)$. Since we now investigated cross-shaped level sets, we showed some function manipulations that keep the cross-shapedness of the level sets: for example the composition with a strictly increasing function.

To even get the Strong Rounding Property in Theorem 3.11 we need to assume additionally that the level sets are also symmetric with respect to the hyperplanes through the continuous minimizer $\bar{x}$ and parallel to the coordinate axes. We showed that this condition is, for symmetric feasible regions, equivalent to the constraint that the objective function $f(x)$ only depends on $\left(\left|x_{1}-\bar{x}_{1}\right|, \ldots,\left|x_{n}-\bar{x}_{n}\right|\right)$.

Since the definition of cross-shapedness and the examples we gave in two dimensions are reminiscent of the definition of biconvexity, we compared in Section 3.3 the definitions of convex, biconvex and ortho-convex sets with the definition of cross-shaped sets. The results are summarized in Figure 3.11 and Figure 3.12.

Section 3.4 gives some examples of problems whose level sets are cross-shaped with respect to the continuous minimizers and who hence have the Rounding Property. A class of objective functions that have (in an unconstrained problem) cross-shaped level sets with respect to any continuous minimizer are for example separable quasiconvex functions. We generalized this to ordered median functions of quasiconvex functions. Next we investigated which properties of a norm $\|\cdot\|$ make sure that the problem $\left(\|x-a\|, \mathbb{R}^{n}\right)$ for some $a \in \mathbb{R}^{n}$ has the Rounding Property: for example if the norm is absolute. We ended the section on applications by investigating which of the median and center problems for the 1-, 2- and $\infty$-norm have the (Strong) Rounding Property.

In the last section we generalized the concept of quasi-roundness to quasi-cross-shapedness. As in the former case we showed in Theorem 3.45 that if the level sets are "not too different" from a cross-shaped set, we get the Rounding Property. 


\section{Comparison and generalizations}

We end this first part by pointing out some differences between the results we achieved for quasi-round and cross-shaped level sets and by giving some common generalizations.

\subsection{Comparison}

Even though we observed a lot of parallelity in the last two chapters there are some differences that we discuss in the following. We start by comparing the basic properties of quasi-round and cross-shaped sets.

First of all, we observe that while the definition of cross-shapedness implies that the set is connected, this is not true for quasi-round sets: also not connected sets can be quasi-round, compare for example Figure 2.1.

Comparing the Lemmas 2.4 and 3.4 we observe that the cross-shapedness of a set is scale invariant and the quasi-roundness is not. This is also the reason why we only allow translation of the input in 2.4 (ii): $g_{4}(x):=f(x-a)$, while we allow in 3.4 (ii) also scaling: $g_{4}(x):=$ $f(\beta x-a)$. This scaling of the input results in scaled level sets which remain cross-shaped but not necessarily quasi-round.

On the other hand, the quasi-roundness for $p=2$ is invariant under rotation, while the cross-shapedness is not, compare Figure 4.1.
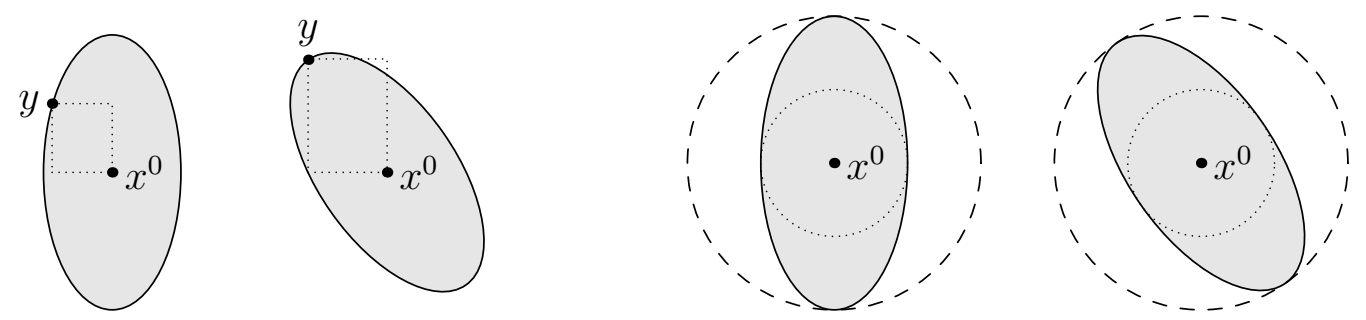

Figure 4.1.: While cross-shapedness is not preserved under rotation (left), quasi-roundness for $p=2$ is (right).

Note however that the invariance of quasi-roundness under rotation is only true for $p=2$, 
compare Figure 4.2 for a counterexample for $p=1$.
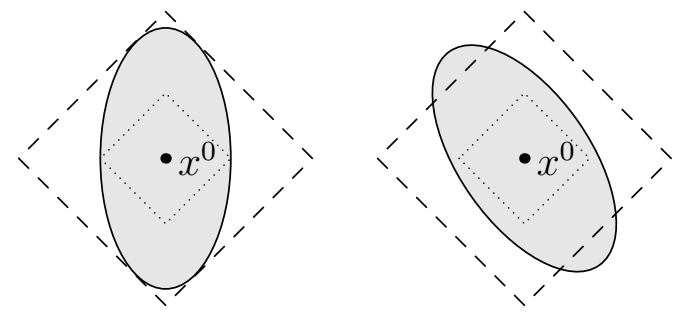

Figure 4.2.: Quasi-roundness of a set is not preserved under rotation for $p=1$.

We point out here that even though the cross-shapedness and the quasi-roundness of a set are invariant under translation the Rounding Property is not, compare Figure 4.3: $\left(p_{M, \bar{x}}, \mathbb{R}^{2}\right)$ has the Rounding Property as $x^{*}=\lfloor\bar{x}\rceil$. But translating $M$ and $\bar{x}$ destroys it: $\left(p_{M+x, \bar{x}+x}, \mathbb{R}^{2}\right)$ does not have the Rounding Property.

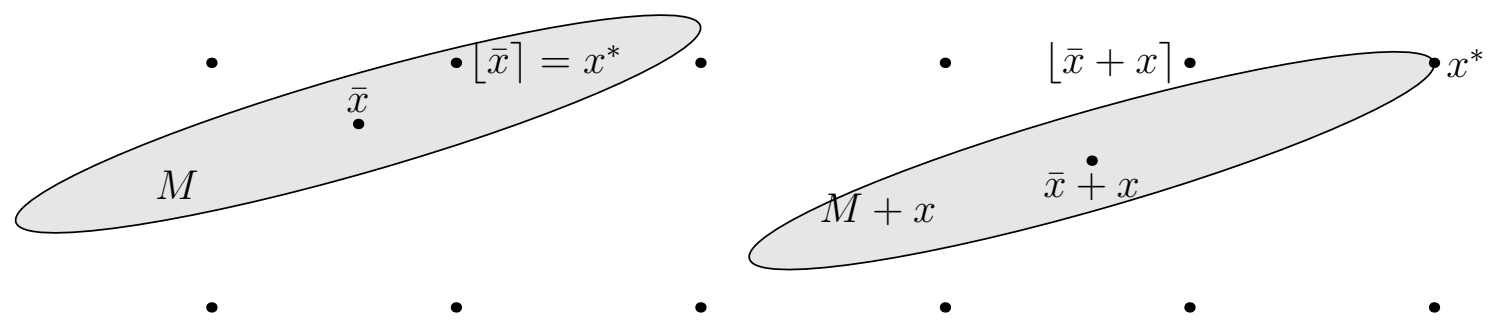

Figure 4.3.: $\left(p_{M, \bar{x}}, \mathbb{R}^{2}\right)$ has the Rounding Property, but $\left(p_{M+x, \bar{x}+x}, \mathbb{R}^{2}\right)$ does not.

The next difference we observe regards the position of $x^{0}$ in a set that is quasi-round or crossshaped w.r.t. $x^{0}$. While (at least for $\max _{x \in M}\left\|x-x^{0}\right\|_{p}>\alpha$ ) the $(\alpha, p)$-quasi-roundness of $M$ w.r.t. $x^{0}$ implies that a small neighborhood of $x^{0}$ is contained in $M$, it is not necessary that $x^{0}$ is an interior point of $M$ in order for $M$ to be cross-shaped w.r.t. $x^{0}$, compare the example in Figure 4.4.

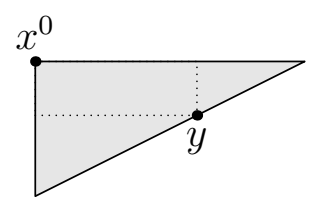

Figure 4.4.: $x^{0}$ need not be in the interior of $M$ in order for $M$ to be cross-shaped w.r.t. $x^{0}$.

The last remark concerns the Strong Rounding Property. One could get the feeling that symmetry plays an important role for the Strong Rounding Property. We point out that even though coordinate axial symmetry in combination with cross-shapedness or in combination with convexity guarantees the Strong Rounding Property (compare Theorem 3.11 and Corollary 3.19), it is not sufficient in the quasi-round case, compare Figure 4.5. 


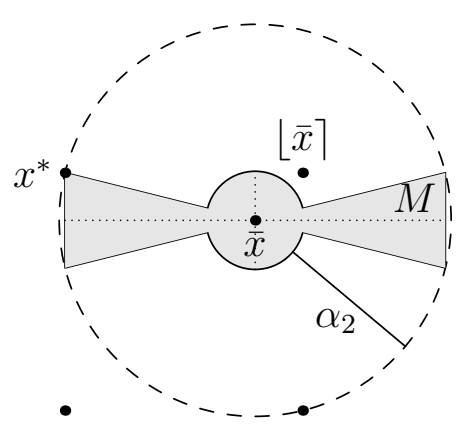

Figure 4.5.: $\left(p_{M, \bar{x}}, \mathbb{R}^{2}\right)$ does not have the Strong Rounding Property even though $M$ is coordinate axially symmetric and $\alpha_{2}$-quasi-round w.r.t. $\bar{x}$.

On the other hand, in order to guarantee the Strong Rounding Property in the quasi-round setting (compare Theorem 2.16) we do not need any symmetry assumptions.

\subsection{Generalization}

In this section we give some generalizations that can be applied to the results in both Chapter 2 and Chapter 3.

First of all we mention that we can relax the assumptions in Theorems 2.10, 3.6 etc. by observing that we do not need the assumed geometric shapes for all level sets where $z \in$ $[f(\bar{x}), \min \{f(x): x \in \operatorname{Round}(\bar{x}) \cap F\}]$ but only for all

$$
z \in[f(\bar{x}), \min \{f(x): x \in \operatorname{Round}(\bar{x}) \cap F\}] \cap f\left(\mathbb{Z}^{n} \cap F\right)
$$

where we define $f\left(\mathbb{Z}^{n} \cap F\right):=\left\{f(x): x \in \mathbb{Z}^{n} \cap F\right\}$. We formulate this for Theorem 3.45, as we mentioned before, this is the general form that contains all others as special cases.

Theorem 4.1 (Rounding Property for quasi-cross-shaped sets II). Let $(f, F)$ be given. Assume that for any optimal solution $\bar{x}$ to $(C P)$ and for any level $z \in[f(\bar{x}), \min \{f(x): x \in$ $\operatorname{Round}(\bar{x}) \cap F\}] \cap f\left(\mathbb{Z}^{n} \cap F\right)$ the level set $\mathcal{L}_{\leq}^{F}(z)$ is $\epsilon(A, \bar{x}, F)$-quasi-cross-shaped w.r.t. $\bar{x}$ where

$$
\epsilon(A, \bar{x}, F):=\min _{x \in\left(\mathbb{Z}^{n} \cap F\right) \backslash \operatorname{Round}(\bar{x})} p_{A, \bar{x}}(x)-\min _{x \in \mathbb{Z}^{n} \cap F} p_{A, \bar{x}}(x) .
$$

Then $(f, F)$ has the Rounding Property.

Proof. This proof is the same as the one of Theorem 3.45: note that we only used the quasicross-shapedness of $\mathcal{L}_{\leq}^{F}(f(x))$ where $x \in \mathbb{Z}^{n} \cap F$.

In fact, it is even enough if the assumption holds for $f\left(x^{*}\right):=\min \left\{f(x): x \in \mathbb{Z}^{n} \cap F\right\}$, but as we do not know $x^{*}$ this assumption is probably hard to check. Therefore we formulated the results with the stricter assumption that all level sets for $z \in[f(\bar{x}), \min \{f(x): x \in \operatorname{Round}(\bar{x})\}]$ have the appropriate shape. The assumption we posed in Theorem 4.1 is a compromise between these two extreme ways to formulate our results. It might be helpful to have Theorem 4.1 in this way if $\mid\left[f(\bar{x}, \min \{f(x): x \in \operatorname{Round}(\bar{x})\}] \cap f\left(\mathbb{Z}^{n} \cap F\right) \mid\right.$ is very small and we can determine 
$\mathcal{L}_{\leq}(z)$ explicitly for each of these levels. Nevertheless, we continue to formulate our results for all $z \in[f(\bar{x}), \min \{f(x): x \in \operatorname{Round}(\bar{x})\}]$ in the following - just keep in mind that we can always perform this relaxation, if helpful.

As mentioned before we do not even need $A$ to be cross-shaped in Theorem 3.45.

Theorem 4.2 (Rounding Property for $\epsilon$-similar sets). Let $(f, F)$ be given. Assume that for any optimal solution $\bar{x}$ to $(C P)$ and for any level $z \leq \min \{f(x): x \in \operatorname{Round}(\bar{x}) \cap F\}$ there exists a set $A$ such that $\left(p_{A, \bar{x}}, \mathbb{R}^{n}\right)$ has the Rounding Property and such that the level set $\mathcal{L}_{\leq}^{F}(z)$ is $\epsilon(A, \bar{x}, F)$-similar to $A$ w.r.t. $\bar{x}$ where

$$
\epsilon(A, \bar{x}, F):=\min _{x \in\left(\mathbb{Z}^{n} \cap F\right) \backslash \operatorname{Round}(\bar{x})} p_{A, \bar{x}}(x)-\min _{x \in \mathbb{Z}^{n} \cap F} p_{A, \bar{x}}(x) .
$$

Then $(f, F)$ has the Rounding Property.

Proof. This proof is the same as the one of Theorem 3.45: note that we only used that $\left(p_{A, \bar{x}}, \mathbb{R}^{n}\right)$ has the RP and not explicitly that $A$ is cross-shaped.

As before, it is very important to note here that $\epsilon(A, \bar{x}, F)$ depends highly on $A$ and is going to be 0 for many sets. Nevertheless, there are of course examples where Theorem 4.2 adds some new insights, compare also the following example.

Example 4.3. $\left(p_{M, \bar{x}}, \mathbb{R}^{2}\right)$ with the set $M$ as in Figure 4.6 obviously has the Rounding Property since $p_{M, \bar{x}}(\lfloor\bar{x}\rceil) \leq p_{M, \bar{x}}(x)$ for all $x \in \mathbb{Z}^{2}$ and hence $X^{*} \cap \operatorname{Round}(\bar{x}) \neq \emptyset$. Furthermore, $M$ fulfills the assumptions of Theorem 4.2 if we choose $A$ as depicted in Figure 4.6 (note that $\left(p_{A, \bar{x}}, \mathbb{R}^{2}\right)$ also has the RP). But $M$ is not quasi-cross-shaped: if there would be a cross-shaped set $A^{\prime}$ such that $M \subseteq A^{\prime}$ (we set $\lambda_{2}=1$ without loss of generality here), we need to have $\lfloor\bar{x}\rceil \in$ $\lambda_{1}(A-\bar{x})+\bar{x}$ by definition of $\epsilon(A, \bar{x}, F)$. But this would mean $[\lfloor\bar{x}\rceil, \bar{x}]_{1} \subseteq \lambda_{1}(A-\bar{x})+\bar{x} \subseteq M$ as $\lambda_{1}(A-\bar{x})+\bar{x}$ is also cross-shaped w.r.t. $\bar{x}$ (compare Lemma 3.4). This is however not true as can be seen in Figure 4.6.

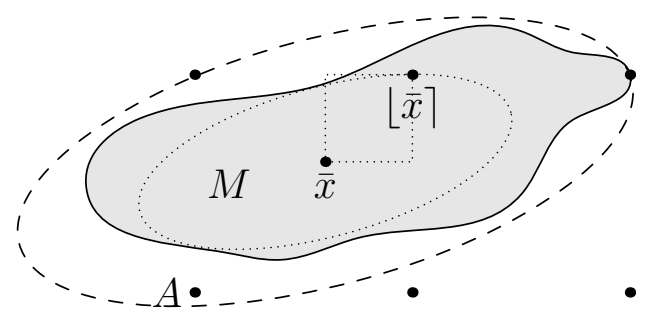

Figure 4.6.: An example for a level set whose Rounding Property can be shown by Theorem 4.2 but not by Theorem 3.45 .

Furthermore we can adapt Theorem 4.2 in an obvious way to guarantee the Strong Rounding Property. 
Theorem 4.4 (Strong Rounding Property for $\epsilon$-similar sets). Let $(f, F)$ be given. Assume that for any optimal solution $\bar{x}$ to $(C P)$ we have that $\lfloor\bar{x}\rceil \in F$ and for any level $f(\bar{x}) \leq$ $z \leq f(\lfloor\bar{x}\rceil)$ there exists a set $A$ such that $\left(p_{A, \bar{x}}, \mathbb{R}^{n}\right)$ has the Strong Rounding Property and such that the level set $\mathcal{L}_{\leq}^{F}(z)$ is $\eta(A, \bar{x}, F)$-similar to $A$ w.r.t. $\bar{x}$ where

$$
\eta(A, \bar{x}, F):=\min _{x \in\left(\mathbb{Z}^{n} \cap F\right) \backslash\lfloor\bar{x}\rceil} p_{A, \bar{x}}(x)-\min _{x \in \mathbb{Z}^{n} \cap F} p_{A, \bar{x}}(x) .
$$

Then $(f, F)$ has the Strong Rounding Property.

Proof. Let $\bar{x} \in \bar{X}$ and $x \in \mathbb{Z}^{n} \cap F$. Using Lemma 1.18 we show that $\lfloor\bar{x}\rceil \in \mathcal{L}_{\leq}^{F}(f(x))$. If $f(\lfloor\bar{x}\rceil) \leq f(x)$ we are done. Otherwise we have

(i) $x \neq\lfloor\bar{x}\rceil$ and

(ii) there exists a set $A \subseteq \mathbb{R}^{n}$ such that $\left(p_{A, \bar{x}}, \mathbb{R}^{n}\right)$ has the Strong Rounding Property and such that $\mathcal{L}_{\leq}^{F}(f(x))$ is $\eta(A, \bar{x}, F)$-similar to $A$ w.r.t. $\bar{x}$, i.e. there exist $\lambda_{1}, \lambda_{2} \in \mathbb{R}_{0}^{+}$s.t.

$$
\lambda_{1}(A-\bar{x})+\bar{x} \subseteq \mathcal{L}_{\leq}^{F}(f(x)) \subseteq \lambda_{2}(A-\bar{x})+\bar{x}
$$

and $\lambda_{2}-\lambda_{1} \leq \eta(A, \bar{x}, F)$.

As $x \in \mathcal{L}_{\leq}^{F}(f(x))$ we have $p_{A, \bar{x}}(x) \leq \lambda_{2}$. Since $\left(p_{A, \bar{x}}, \mathbb{R}^{n}\right)$ has the Strong Rounding Property we have that $\lfloor\bar{x}\rceil \in \operatorname{argmin}_{y \in \mathbb{Z}^{n}} p_{A, \bar{x}}(y)$. Hence we have $\min _{y \in \mathbb{Z}^{n} \cap F} p_{A, \bar{x}}(y)=p_{A, \bar{x}}(\lfloor\bar{x}\rceil)$ and thus

$$
\begin{aligned}
p_{A, \bar{x}}(\lfloor\bar{x}\rceil) & =\min _{y \in\left(\mathbb{Z}^{n} \cap F\right) \backslash\lfloor\bar{x}\rceil} p_{A, \bar{x}}(y)-\eta(A, \bar{x}, F) \\
& \leq p_{A, \bar{x}}(x)-\eta(A, \bar{x}, F) \quad \text { as } x \in \mathbb{Z}^{n} \cap F, x \neq\lfloor\bar{x}\rceil \\
& \leq \lambda_{2}-\eta(A, \bar{x}, F) \leq \lambda_{1} .
\end{aligned}
$$

And therefore $\lfloor\bar{x}\rceil \in \lambda_{1}(A-\bar{x})+\bar{x} \subseteq \mathcal{L}_{\leq}^{F}(f(x))$ and thus $f(\lfloor\bar{x}\rceil) \leq f(x)<f(\lfloor\bar{x}\rceil)$. Like this the assumption $f(x)<f(\lfloor\bar{x}\rceil)$ leads to a contradiction.

As already mentioned in Section 1.2 the definition of the Rounding Property might seem too restrictive: is it not enough if there is one continuous minimizer $\bar{x}$ that fulfills $\operatorname{Round}(\bar{x}) \cap X^{*} \neq$ $\emptyset$ ? As mentioned before (compare Section 1.2) we want the Rounding Property for any optimal solution $\bar{x} \in \bar{X}$ because we assume in general the existence of a black-box algorithm to solve $(C P)$ and therefore we do not know how to get a specific solution and we have to assume that it is more expensive to get a specific one. Nevertheless, there might be situations where we are indeed able to get a specific continuous minimizer. Therefore we define and discuss the Weakened Rounding Property in the following.

Definition 4.5. We say that an integer problem $(I P)$ has the Weakened Rounding Property if there exists an optimal solution $\bar{x} \in \bar{X}$ to its continuous relaxation $(C P)$ such that $X^{*} \cap \operatorname{Round}(\bar{x}) \neq \emptyset$.

First of all we observe the following simple connection between the Rounding Property and the Weakened Rounding Property. 
Lemma 4.6. If an integer problem $(f, F)$ has the Rounding Property it also has the Weakened Rounding Property.

Proof. This is a direct consequence of the definition of the Weakened Rounding Property since we always assume $\bar{X}$ to be nonempty.

Furthermore, all results we had so far can be weakened such that they guarantee the Weakened Rounding Property instead of the Rounding Property. We show this exemplarily for Theorem 4.2.

Theorem 4.7 (Weakened Rounding Property for $\epsilon$-similar sets). Let $(f, F)$ be given. Assume that there exists an optimal solution $\bar{x}$ to $(C P)$ such that for any level $z \in$ $\min \{f(x): x \in \operatorname{Round}(\bar{x}) \cap F\}$ there exists a set $A$ such that $\left(p_{A, \bar{x}}, \mathbb{R}^{n}\right)$ has the Rounding Property and the level set $\mathcal{L}_{\leq}^{F}(z)$ is $\epsilon(A, \bar{x}, F)$-similar to $A$ w.r.t. $\bar{x}$ where

$$
\epsilon(A, \bar{x}, F):=\min _{x \in\left(\mathbb{Z}^{n} \cap F\right) \backslash \operatorname{Round}(\bar{x})} p_{A, \bar{x}}(x)-\min _{x \in \mathbb{Z}^{n} \cap F} p_{A, \bar{x}}(x) .
$$

Then $(f, F)$ has the Weakened Rounding Property.

Proof. Let $\bar{x} \in \bar{X}$ as assumed in the theorem and $x \in \mathbb{Z}^{n} \cap F$.

Then the proof follows as in the proof of Theorem 3.45.

An example for a function that has the Weakened Rounding Property but not the Rounding

Property can be found in Figure 4.7.

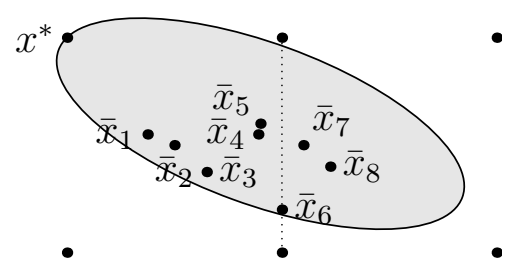

Figure 4.7.: The function whose level sets are depicted here does not have the Rounding Property, but the Weakened Rounding Property.

Assume that $\bar{X}=\left\{\bar{x}_{1}, \ldots, \bar{x}_{8}\right\}$ as depicted in Figure 4.7 and $\mathcal{L}_{\leq}\left(f\left(x^{*}\right)\right)$ is the gray set in Figure 4.7. Then the integer problem of minimizing this function over the integer lattice has the Weakened Rounding Property as $X^{*} \cap \operatorname{Round}\left(\bar{x}_{i}\right) \neq \emptyset$ for $i \in\{1,2,3,4,5\}$ but not the Rounding Property, since $X^{*} \cap \operatorname{Round}\left(\bar{x}_{i}\right)=\emptyset$ for $i \in\{6,7,8\}$.

Analogously we can adopt this concept to the Strong Rounding Property and get the Weakened Strong Rounding Property.

Definition 4.8. We say that an integer problem $(I P)$ has the Weakened Strong Rounding Property if there exists an optimal solution $\bar{x} \in \bar{X}$ to its continuous relaxation $(C P)$ such that $\lfloor\bar{x}\rceil \in X^{*}$. 
Again we can adopt the results we had so far for the Strong Rounding Property for the Weakened Strong Rounding Property. We skip this here since it does not give us any new insights. Instead we show in Figure 4.8 an example situation that has the Weakened Strong Rounding Property and the Rounding Property. This means that for all $\bar{x} \in \bar{X}$ we have $X^{*} \cap \operatorname{Round}(\bar{x}) \neq \emptyset$ and there exists at least one $\hat{x} \in \bar{X}$ such that $\lfloor\hat{x}\rceil \in X^{*}$.

In Figure 4.8 the dark gray area is $\bar{X}, X^{*}=\left\{x^{*}\right\}$ and the light gray area is $\mathcal{L}_{\leq}\left(f\left(x^{*}\right)\right)$. We see that the problem has the Rounding Property since $x^{*} \in \operatorname{Round}(\bar{x})$ for all $\bar{x} \in \bar{X}$ and the Weakened Strong Rounding Property since for all $\bar{x} \in \bar{X} \cap\left\{x \in \mathbb{R}^{2}: x_{2} \geq 0.5\right\}$ we also have $\lfloor\bar{x}\rceil=x^{*}$.

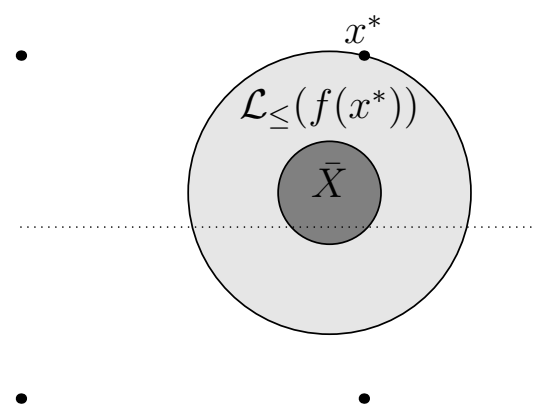

Figure 4.8.: The function whose level sets are depicted here has the Rounding Property and the Weakened Strong Rounding Property. 

Part II.

Applications and extensions 



\section{Lower bounds and algorithms for convex quadratic integer programs}

In this chapter we apply our theory of the Rounding Property to the special case of unconstrained convex quadratic integer programs, i.e., to problems of the form

$$
\begin{array}{lll}
(C Q I P) & \min & q(x):=x^{t} Q x+L^{t} x \\
& \text { s.t. } & x \in \mathbb{Z}^{n}
\end{array}
$$

where $Q \in \mathbb{R}^{n, n}$ is a positive definite matrix and $L \in \mathbb{R}^{n}$. This means that the objective function $q: \mathbb{R}^{n} \rightarrow \mathbb{R}$ is strictly convex.

This chapter is organized as follows. First we recall some facts and notations about convex quadratic integer problems and give a short literature review on their solution. Then we identify properties (of $Q$ ) that lead to cross-shaped or quasi-round level sets. In the next section we consider convex quadratic integer problems that do not have the Rounding Property. In order to solve them via a branch-and-bound approach we underestimate the objective function by another convex quadratic function that has the Strong Rounding Property in order to get an easy to calculate lower bound. Then we show how to achieve good underestimators independent of the continuous minimizer, since we hope that they perform good in a branchand-bound framework. In the last section we compare the different bounds theoretically, in an example and in some computational results.

\subsection{Convex quadratic integer programs}

The special type of problem $(C Q I P)$ has two properties that make it a good application for our theory: first of all, the continuous minimizer is unique since $q$ is strictly convex and can be calculated directly via

$$
\bar{x}=-\frac{1}{2} Q^{-1} L .
$$

And secondly we know what the level sets of $q$ look like:

$$
\mathcal{L}_{\leq}(z)=\sqrt{z-q(\bar{x})} E(Q, \bar{x}) \quad \forall z \geq q(\bar{x})
$$


where $E(Q, \bar{x})$ is the ellipsoid given by $Q$ and centered at $\bar{x}$ :

$$
E(Q, \bar{x}):=\left\{y \in \mathbb{R}^{n}:(y-\bar{x})^{t} Q(y-\bar{x}) \leq 1\right\}
$$

and $r \cdot E(Q, \bar{x}):=\bar{x}+r \cdot E(Q, 0)$ for all $r \geq 0$. (We adopt these notations and results of Buchheim et al. [2012a].)

Note here that this means that the shape of the level sets only depends on $Q$ and not on $L$. This is due to the fact that, using $\bar{x}$, we can reformulate $q$ as

$$
q(x)=q(\bar{x})+(x-\bar{x})^{t} Q(x-\bar{x}) \quad \forall x \in \mathbb{R}^{n} .
$$

Example 5.1. Consider for example the problem

$$
\begin{array}{ccc}
(C Q I P) & \min & x^{t}\left(\begin{array}{rr}
5 & -1 \\
-1 & 5
\end{array}\right) x+\left(\begin{array}{r}
3.4 \\
-7.4
\end{array}\right)^{t} x \\
\text { s.t. } & x \in \mathbb{Z}^{n} .
\end{array}
$$

Here we get $\bar{x}=\left(\begin{array}{r}-0.2 \\ 0.7\end{array}\right)$ and $q(\bar{x})=-2.93$. The level sets for $z=-2.73, z=q\left(x^{*}\right)=-2.4$ and $z=-1.93$ are shown in Figure 5.1. We see that $x^{*}=\lfloor\bar{x}\rceil=\left(\begin{array}{l}0 \\ 1\end{array}\right)$, i.e., q has the SRP.

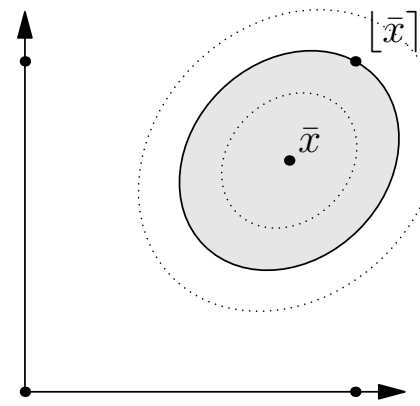

Figure 5.1.: Level sets of a convex quadratic function having the rounding property.

But of course not all convex quadratic integer problems have the Rounding Property, see for example Figure 5.2.

The remainder of this chapter is organized as follows: we end this introduction by providing a short literature review on the special case of integer quadratic optimization and introducing some notations and basic properties of ellipsoids. In the next section we investigate under which assumptions on $Q$ the level sets $\sqrt{z-q(\bar{x})} E(Q, \bar{x})$ are cross-shaped or quasi-round w.r.t. $\bar{x}$. Furthermore, we introduce a special case of quasi-cross-shapedness namely quasiaxisparallelity and investigate under which assumptions the level sets are quasi-axisparallel w.r.t. $\bar{x}$. Since a general convex quadratic integer problem is probably not going to have the Rounding Property we study in the next section how we can derive lower bounds to use in a branch-and-bound framework from the concepts we had so far. To use them efficiently in a branch-and-bound algorithm it might be useful to make their computation independent of $\bar{x}$. This is done in the second to last section. In the last section we compare the bounds we derived in this chapter theoretically, in an example and by some computational experiments. 


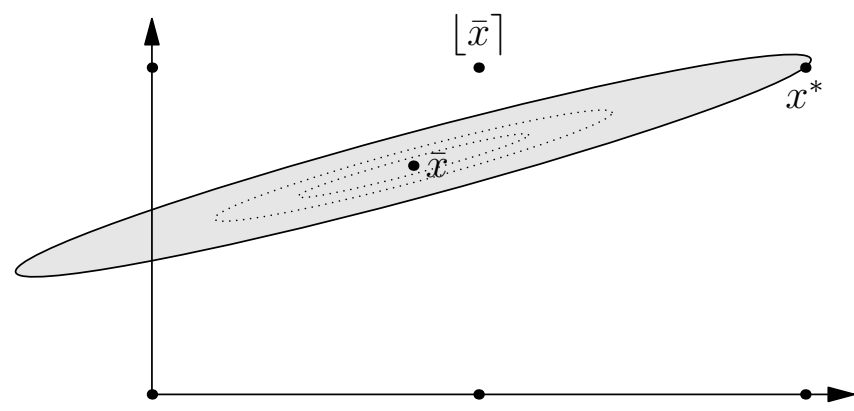

Figure 5.2.: Level sets of a convex quadratic function not having the rounding property.

Besides the fact that problem $(C Q I P)$ fits nicely into our theory it is also a very interesting problem. First of all, we have to mention that even though the continuous relaxation is easy to solve the integer problem $(C Q I P)$ is NP-hard as it is equivalent to the closest vector problem, which is known to be NP-hard (compare van Emde-Boas [1981]). This means that algorithms for the closest vector problem as the Fincke-Pohst algorithm (see Fincke and Pohst [1985]) can also be used to solve $(C Q I P)$. A recent approach to solve $(C Q I P)$ is a branch-and-bound approach by Buchheim et al. [2012a].

Literature on integer quadratic (not necessarily convex) problems is mainly on binary quadratic problems. For this special case it is shown in Carter [1984] that also the problem where $Q$ is indefinite can equivalently be formulated as a convex quadratic binary problem by replacing the matrix $Q$ by a positive definite matrix of the same size. Also the problem over the binary variables is NP-hard as it equivalent to the max-cut problem (see Barahona et al. [1989]). The approaches to solve this problem presented here fall into two groups: the first basic idea is to reformulate the problem as an equivalent problem which is either easier to solve, whose continuous relaxation is easier to solve or gives better bounds or that is for some other reason preferable. The second approach is to use a branch-and-bound framework. Note however that these two classes are not disjoint: often a reformulation is used within or before a branch-andbound algorithm.

The probably most common reformulation strategy in the binary case is the linearization of the objective function: every product $x_{i} \cdot x_{j}$ is replaced by a new variable $y_{i j}$ that is equal to 1 if and only if $x_{i}=x_{j}=1$ (Fortet [1960]). This means that the number of variables and constraints increases by $O\left(n^{2}\right)$. Furthermore, the continuous relaxation of this problem is weak. Therefore a lot of effort has been put into reducing the number of variables (e.g. Glover [1975]) and getting tighter relaxations (e.g. Adams et al. [2004], Adams and Sherali [1986]). Another idea is to use $q_{u}(x):=x^{t}(Q-\operatorname{Diag}(u)) x+(L+u)^{t} x$ instead of $q(x)$. It is obvious that $q_{u}(x)=q(x)$ for all $x \in\{0,1\}^{n}$ and hence the problems are equivalent. If $u$ is chosen in such a way that $Q-\operatorname{Diag}(u) \succeq 0$ this yields a convex quadratic reformulation (compare e.g. Hammer and Rubin [1970], Carter [1984] or McBride and Yormark [1980]). This idea is further improved by Billionnet and Elloumi [2007] and Billionnet et al. [2009] and can be generalized to general mixed- integer programs (Billionnet et al. [2012]).

In addition to and often combined with these reformulation techniques there are a lot of branchand-bound approaches, compare Pardalos and Rodgers [1990] for an overview. We mention two more recent approaches here. They differ mainly in the dual bound-computation. The first 
approach is by Helmberg and Rendl [1998]: the main idea is to reformulate $\min \left\{x^{t} Q x: x \in\right.$ $\left.\{-1,1\}^{n}\right\}$ as $\min \left\{\operatorname{tr}(Q X): X \in \mathcal{P}_{\mathcal{C}}\right\}$ (where $\mathcal{P}_{\mathcal{C}}:=\operatorname{conv}\left\{x x^{t}: x \in\{-1,1\}^{n}\right\}$ is the socalled cut polytope and $\operatorname{tr}(A)$ denotes the trace of a matrix $A$ ) and then use the semidefinite relaxation $\min \{\operatorname{tr}(Q X): \operatorname{diag}(X)=e, X \succeq 0\}$ (where $\operatorname{diag}(A)$ is the $n$ dimensional vector containing the diagonal components of $A$ ) as introduced e.g. by Rendl [1999]. This relaxation is tightened by some hypermetric inequalities, i.e., by inequalities of type $\operatorname{tr}\left(\left(b b^{t}\right)\left(x x^{t}\right)\right) \geq 1$ for $b \in\left\{y \in \mathbb{Z}^{n}: \min \left\{\left|y^{t} x\right|: x \in\{-1,1\}^{n}\right\}=1\right\}$ and solved by interior point methods. The authors argue that this approach is practical for instances with up to 100 binary variables. In the meantime it has been improved by Helmberg [2000] and Rendl et al. [2010].

A completely different approach is presented in a recent paper by Buchheim and Traversi [2013]: in order to solve $\min \left\{q(x):=x \top Q x+L^{t} x: x \in\{0,1\}^{n}\right\}$ they underestimate the objective function $q$ globally by a separable but not necessarily convex quadratic function $g_{z}^{(t)}(x):=(x-z)^{t} \operatorname{Diag}(t)(x-z)+(L+2 Q z)^{t} x-z^{t} Q z$ for a fixed point $z$. In this point $g_{z}^{(t)}$ touches $q: g_{z}^{(t)}(z)=q(z)$ and $g_{z}^{(t)}$ is a global underestimator of $q$ if and only if $Q \succeq \operatorname{Diag}(t)$. The separable quadratic function can then by linearized by using the fact that $x_{i}^{2}=x_{i}$ for $x_{i} \in\{0,1\}$. Thus calculating the lower bounds means minimizing a linear function over the set $\{0,1\}^{n}$. The challenge in this approach is to identify a good underestimator $g_{z}^{(t)}$.

We end this short literature review by mentioning some work on the quadratic integer optimization problem where the variables need not be binary. In this case, as mentioned before, even the continuous relaxation over box constraints, the so-called BoxQP-problem, is NP-hard (see Pardalos and Vavasis [1991]). Some approaches to solve the integer non-convex quadratic problem include convex reformulation (as done in Billionnet et al. [2013]), d.c. decomposition (see Bomze and Locatelli [2004]), ellipsoidal approximation (compare Buchheim et al. [2012b]) and SDP-relaxation (Buchheim and Wiegele [2012]). Proximity results for separable quadratic integer problems are given in Granot and Skorin-Kapov [1990].

To end this introduction we give some notations and some basic properties of ellipsoids that we use in the following.

Notation 5.2. (i) We denote by $Q \succeq 0$ that $Q$ is positive semidefinite and by $Q \succ 0$ that $Q$ is positive definite. Both notations hence imply that $Q$ is symmetric.

(ii) We define $E(Q, x):=\left\{y \in \mathbb{R}^{n}:(y-x)^{t} Q(y-x) \leq 1\right\}$ also for $Q \succeq 0$. Note however that this set is unbounded if $Q$ is not positive definite, but we still call it an "ellipsoid".

(iii) We define $\|x\|_{Q}:=\sqrt{x^{t} Q x}$ for $Q \succeq 0$, which is a norm if $Q \succ 0$.

Remark 5.3. (i) $E(Q, x)=x+E(Q, 0)$.

(ii) $r \cdot E(Q, x)=E\left(\frac{1}{r^{2}} Q, x\right)$. Note once again here that $r \cdot E(Q, x):=x+r \cdot E(Q, 0)$ for all $r \geq 0$ which does not correspond to the usual notation $r \cdot M$. We use this notation here since it abbreviates the notation a lot in the following.

(iii) The eigenvectors of $Q$ define the principal directions of $E(Q, 0)$ and the reciprocal square roots of the eigenvalues are the equatorial radii.

(iv) $x \in R \cdot E\left(Q, x^{0}\right)$ if and only if $\left\|x-x^{0}\right\|_{Q} \leq R$. 


\subsection{Axisparallel, quasi-round and quasi-axisparallel ellipsoids}

Since $\bar{x}=-\frac{1}{2} Q^{-1} L$ is the unique continuous minimizer we investigate in this section under which assumptions the level sets, which are of the form $r \cdot E(Q, \bar{x})$, are cross-shaped, quasiround or quasi-axisparallel w.r.t. $\bar{x}$. (Quasi-axisparallelity is a special case of quasi-crossshapedness and will be defined in this section.)

First we investigate the case of cross-shaped ellipsoids. Since cross-shapedness is invariant under translation and scaling (compare Lemma 3.4) we ask: which properties of $Q$ make sure that $E(Q, 0)$ is cross-shaped w.r.t. 0 ? We state the following lemma in a more general way, namely not only for positive definite matrices $Q$ but for positive semidefinite matrices $Q$.

Lemma 5.4. The ellipsoid $E(Q, 0)$ where $Q$ is positive semidefinite is cross-shaped w.r.t. 0 if and only if $Q=\operatorname{Diag}(t)$ for some $t \geq 0$.

Proof. We aim at showing that $Q=\operatorname{Diag}(t)$ for some $t \geq 0$ if and only if $[y, 0]_{1} \subseteq E(Q, 0)$ for all $y \in E(Q, 0)$, where $[y, 0]_{1}=\left\{p \in \mathbb{R}^{n}: p_{i}=\lambda_{i} y_{i}, \lambda_{i} \in[0,1] \forall i=1, \ldots, n\right\}$.

" $\Leftarrow$ " Let $Q=\operatorname{Diag}(t)$ for some $t \geq 0$, this means $E(Q, 0)=\left\{x \in \mathbb{R}^{n}: \sum_{i=1}^{n} t_{i} x_{i}^{2} \leq 1\right\}$. Let $y \in E(Q, 0)$, thus $\sum_{i=1}^{n} t_{i} y_{i}^{2} \leq 1$, and $p \in[y, 0]_{1}$. Then

$$
\sum_{i=1}^{n} t_{i} p_{i}^{2}=\sum_{i=1}^{n} \underbrace{t_{i}}_{\geq 0} \underbrace{\lambda_{i}^{2}}_{\leq 1} \underbrace{y_{i}^{2}}_{\geq 0} \leq \sum_{i=1}^{n} t_{i} y_{i}^{2} \leq 1 .
$$

This means, $[y, 0]_{1} \subseteq E(Q, 0)$ for all $y \in E(Q, 0)$.

“ $\Rightarrow$ Let $E(Q, 0)=\left\{x \in \mathbb{R}^{n}: \sum_{i=1}^{n} \sum_{j=1}^{n} q_{i j} x_{i} x_{j} \leq 1\right\}$ be cross-shaped w.r.t. 0 and assume that $Q \neq \operatorname{Diag}(t)$ for any $t \geq 0$, i.e., there exist indices $l \neq k$ such that $q_{k l} \neq 0$. By $Q \succeq 0$, we have $q_{k k} \geq 0, q_{l l} \geq 0, q_{k k} q_{l l}-q_{k l}^{2} \geq 0$. Using $q_{k l}^{2}>0$, this implies

$$
q_{k k}>0, \quad q_{l l}>0, \quad 4 q_{k k} q_{l l}-q_{k l}^{2}>4\left(q_{k k} q_{l l}-q_{k l}^{2}\right) \geq 0 .
$$

Now we construct $y \in E(Q, 0)$ with $[y, 0]_{1} \nsubseteq E(Q, 0)$, in contradiction to our assumption. Define $y \in \mathbb{R}^{n}$ by

$$
y_{i}= \begin{cases}0 & \text { if } i \notin\{k, l\} \\ \frac{2 \sqrt{q_{l l}}}{\sqrt{4 q_{k k} q_{l l}-q_{k l}^{2}}} & \text { if } i=k \\ -\frac{q_{k l}}{\sqrt{q_{l l}} \sqrt{4 q_{k k} q_{l l}-q_{k l}^{2}}} & \text { if } i=l .\end{cases}
$$

The vector $y$ is well-defined by (5.1) and we have $y \neq 0$. We obtain

$$
\begin{aligned}
\sum_{i, j=1}^{n} q_{i j} y_{i} y_{j} & =q_{k k} \cdot \frac{4 q_{l l}}{4 q_{k k} q_{l l}-q_{k l}^{2}}+q_{l l} \cdot \frac{q_{k l}^{2}}{q_{l l}\left(4 q_{k k} q_{l l}-q_{k l}^{2}\right)}-2 q_{k l} \cdot \frac{2 \sqrt{q_{l l}} q_{k l}}{\sqrt{q_{l l}}\left(4 q_{k k} q_{l l}-q_{k l}^{2}\right)} \\
& =\frac{4 q_{k k} q_{l l}-3 q_{k l}^{2}}{4 q_{k k} q_{l l}-q_{k l}^{2}}<1
\end{aligned}
$$


and thus $y \in E(Q, 0)$. However, for $p$ with $p_{i}=\left\{\begin{array}{ll}y_{k} & \text { if } i=k \\ 0 & \text { otherwise }\end{array}\right.$ we have $p \in[y, 0]_{1}$, but

$$
\sum_{i, j=1}^{n} q_{i j} p_{i} p_{j}=q_{k k} y_{k}^{2}=\frac{4 q_{k k} q_{l l}}{4 q_{k k} q_{l l}-q_{k l}^{2}}>1
$$

as $4 q_{k k} q_{l l}-q_{k l}^{2}>0$ and $q_{k l}^{2}>0$. This means, $p \notin E(Q, 0)$ and therefore $[y, 0]_{1} \not \subset E(Q, 0)$.

Examples for a cross-shaped ellipsoid and an ellipsoid that is not cross-shaped can be seen in Figure 5.3.
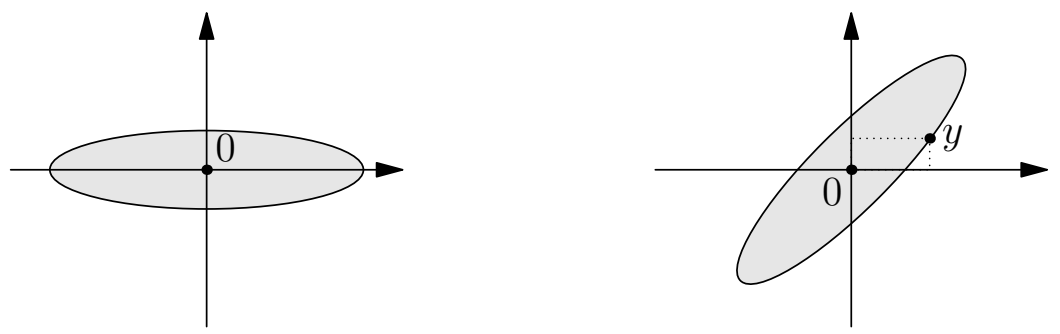

Figure 5.3.: Left: cross-shaped w.r.t. 0, right: not cross-shaped w.r.t. 0.

Notation 5.5. If $Q=\operatorname{Diag}(t)$ for some $t \geq 0$ the ellipsoid is called axisparallel.

We use the name axisparallel in the following since it suggests the right picture. Axisparallel ellipsoids are not only cross-shaped but also coordinate axially symmetric with respect to their center. Therefore we get the following lemma.

Lemma 5.6. If $Q=\operatorname{Diag}(t)$ for some $t \geq 0$, problem $(C Q I P)$ has the Strong Rounding Property and an optimal solution is given by $\left\lfloor-\frac{1}{2} Q^{-1} L\right\rceil$.

Proof. If $Q=\operatorname{Diag}(t)$ for some $t \geq 0$ the level sets are cross-shaped (compare Lemma 5.4) and coordinate axially symmetric w.r.t. $\bar{x}=-\frac{1}{2} Q^{-1} L$ : again it is enough to prove that $E(\operatorname{Diag}(t), 0)$ is cas w.r.t. 0 and this follows since $E(\operatorname{Diag}(t), 0)=\left\{x \in \mathbb{R}^{n}: \sum_{i=1}^{n} t_{i} x_{i}^{2} \leq 1\right\}$, i.e., if $y \in E(\operatorname{Diag}(t), 0)$, also $y^{i}$ defined by $y_{j}^{i}=\left\{\begin{array}{ll}y_{j} & \text { if } j \neq i \\ -y_{i} & \text { otherwise }\end{array}\right.$ is in $E(\operatorname{Diag}(t), 0)$ for all $i \in\{1, \ldots, n\}$. Therefore Theorem 3.11 proves that $\left(q, \mathbb{R}^{n}\right)$ has the SRP and hence we know that $\lfloor\bar{x}\rceil \in X^{*}$.

Remark 5.7. The fact that the level sets of $q$ are cross-shaped w.r.t. $\bar{x}$ for $Q=\operatorname{Diag}(t)$ for some $t \geq 0$ can also be proven by either of the following observations:

(i) $Q=\operatorname{Diag}(t)$ for some $t \geq 0$ implies that $q$ is of the form $q(x)=\sum_{i=1}^{n}\left(t_{i} x_{i}^{2}+l_{i} x_{i}\right)$ and therefore a separable convex function. Hence the claim follows with Lemma 3.21. 
(ii) If $t>0, E(\operatorname{Diag}(t), 0)$ can also be interpreted as the weighted version of a 2-norm unit ball:

$$
E(\operatorname{Diag}(t), 0):=\{x \in \mathbb{R}^{n}: \sum_{i=1}^{n} \underbrace{t_{i}}_{>0} x_{i}^{2} \leq 1\}=\left\{x \in \mathbb{R}^{n}:\|x\|_{2}^{\sqrt{t}} \leq 1\right\}=B_{\|\cdot\|_{2}^{\sqrt{t}}} .
$$

Consequently the claim follows with Lemma 3.34.

Now we investigate under which assumptions the level sets of the convex quadratic function $q$ are $\left(\alpha_{2}(\bar{x}), 2\right)$-quasi-round w.r.t. the continuous minimizer $\bar{x}$. To do this we analyze first when a given ellipsoid $E(Q, 0)$ is $(\alpha, 2)$-quasi-round w.r.t. 0 for some given $\alpha \geq 0$. Here the generalization to positive semidefinite matrices is not meaningful since only bounded sets can be quasi-round. Furthermore, we only investigate $p=2$ since Euclidean balls seem to be the best choice for approximating ellipsoids.

Lemma 5.8. Let $\alpha \geq 0, x \in \mathbb{R}^{n}$. Let $\lambda_{1}, \ldots, \lambda_{n}$ denote the eigenvalues of $Q$ and let $\lambda_{\min }=$ $\min _{i} \lambda_{i}$ and $\lambda_{\max }=\max _{i} \lambda_{i}$. The ellipsoid $E(Q, 0)$ is $(\alpha, 2)$-quasi-round w.r.t. 0 if and only if

$$
\frac{1}{\sqrt{\lambda_{\min }}}-\frac{1}{\sqrt{\lambda_{\max }}} \leq \alpha
$$

Note that since $Q$ is positive definite we have $\lambda_{\max } \geq \lambda_{\min }>0$.

Proof. Since for ellipsoids the incenter and the circumcenter coincide with the center of the ellipsoid, here 0 , we have that $B_{2}(0, r) \subseteq E(Q, 0)$ if and only if $r \leq r_{i n}$ where $r_{i n}$ is the inradius. Equivalently $E(Q, 0) \subseteq B_{2}(0, R)$ if and only if $R \geq R_{\text {circ }}$ where $R_{\text {circ }}$ is the circumradius.

Furthermore, we know that the inradius and circumradius are given by the smallest and biggest equatorial radius, i.e., $r_{i n}=\frac{1}{\sqrt{\lambda_{\max }}}$ and $R_{c i r c}=\frac{1}{\sqrt{\lambda_{\min }}}$.

Assume now that $E(Q, 0)$ is $(\alpha, 2)$-quasi-round w.r.t. 0 , i.e there exist $r, R \in \mathbb{R}_{0}^{+}$such that $B_{2}(0, r) \subseteq E(Q, 0) \subseteq B_{2}(0, R)$ and $R-r \leq \alpha$. This means that $r \leq r_{i n}$ and $R \geq R_{\text {circ }}$ and therefore $\frac{1}{\sqrt{\lambda_{\min }}}-\frac{1}{\sqrt{\lambda_{\max }}}=R_{\text {circ }}-r_{i n} \leq R-r \leq \alpha$. On the other hand, if $R_{\text {circ }}-r_{\text {in }} \leq \alpha$ we get that $E(Q, 0)$ is $(\alpha, 2)$-quasiround w.r.t 0 since $B_{2}\left(0, r_{i n}\right) \subseteq E(Q, 0) \subseteq B_{2}\left(0, R_{\text {circ }}\right)$.

An example for an $(\alpha, 2)$-quasi-round ellipsoid can be seen in Figure 5.4.

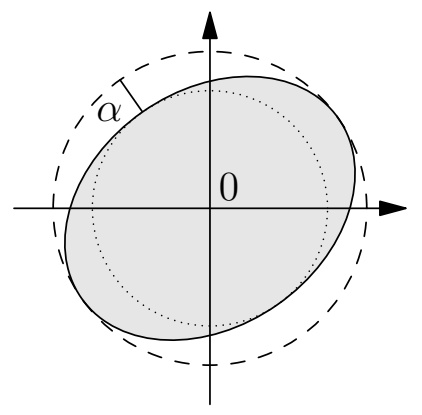

Figure 5.4.: An $(\alpha, 2)$-quasi-round ellipsoid.

As we already mentioned, quasi-roundness is not invariant under scaling and therefore it is not enough to check whether $E(Q, 0)$ is quasi-round but we have to check whether $\sqrt{z-q(\bar{x})}$. $E(Q, \bar{x})$ is $\left(\alpha_{2}(\bar{x}), 2\right)$-quasi-round w.r.t. $\bar{x}$ for all $0 \leq z \leq \min \{q(x): x \in \operatorname{Round}(\bar{x})\}$. 
Lemma 5.9. Let $\bar{x}$ be the unique continuous minimizer to $(C Q I P)$ and choose $\widetilde{x} \in$ $\operatorname{argmin}\{q(x): \quad x \in \operatorname{Round}(\bar{x})\}$. Let $\lambda_{1}, \ldots, \lambda_{n}$ denote the eigenvalues of $Q$ and $\lambda_{\min }:=\min _{i} \lambda_{i}, \lambda_{\max }:=\max _{i} \lambda_{i}$. If

$$
\frac{1}{\sqrt{\lambda_{\min }}}-\frac{1}{\sqrt{\lambda_{\max }}} \leq \frac{\alpha_{2}(\bar{x})}{\sqrt{q(\widetilde{x})-q(\bar{x})}}
$$

$(C Q I P)$ has the Rounding Property.

Proof. If $z=q(\bar{x})$ we know that $\mathcal{L}_{\leq}(q(\bar{x}))=\{\bar{x}\}$ which is quasiround w.r.t. $\bar{x}$ for all $\alpha \geq 0$. For $z>q(\bar{x})$ the level sets are of the form $\mathcal{L}_{\leq}(z)=\sqrt{z-q(\bar{x})} E(Q, \bar{x})=E\left(\frac{1}{z-q(\bar{x})} Q, \bar{x}\right)$. The eigenvalues of $\frac{1}{z-q(\bar{x})} Q$ are $\mu_{i}=\frac{\lambda_{i}}{z-q(\bar{x})}$ for all $i=1, \ldots, n$. This means that $\mu_{\min }=\frac{\lambda_{\min }}{z-q(\bar{x})}$ and $\mu_{\max }=\frac{\lambda_{\max }}{z-q(\bar{x})}$.

If $\frac{1}{\sqrt{\lambda_{\min }}}-\frac{1}{\sqrt{\lambda_{\max }}} \leq \frac{\alpha_{2}(\bar{x})}{\sqrt{q(\widetilde{x})-q(\bar{x})}}$ we know that

$$
\begin{aligned}
& \frac{1}{\sqrt{\lambda_{\min }}}-\frac{1}{\sqrt{\lambda_{\max }}} \leq \frac{\alpha_{2}(\bar{x})}{\sqrt{z-q(\bar{x})}} \quad \forall q(\bar{x})<z \leq q(\widetilde{x}) \\
\Rightarrow & \frac{1}{\sqrt{\mu_{\min }}}-\frac{1}{\sqrt{\mu_{\max }}} \leq \alpha_{2}(\bar{x}) \quad \forall q(\bar{x})<z \leq q(\widetilde{x}) .
\end{aligned}
$$

Using Lemma 5.8 this means that $\mathcal{L}_{\leq}(z)=E\left(\frac{1}{z-q(\bar{x})} Q, \bar{x}\right)$ is $\left(\alpha_{2}(\bar{x}), 2\right)$-quasi-round w.r.t. $\bar{x}$ for all $q(\bar{x}) \leq z \leq q(\widetilde{x})=\min \{q(x): x \in \operatorname{Round}(\bar{x})\}$. Hence Theorem 2.8 makes sure that $(C Q I P)$ has the RP.

As before we get the Strong Rounding Property by replacing $\alpha$ by $\beta$ :

Corollary 5.10. Let $\bar{x}$ be the unique continuous minimizer to $(C Q I P)$. Let $\lambda_{1}, \ldots, \lambda_{n}$ denote the eigenvalues of $Q$ and $\lambda_{\min }:=\min _{i} \lambda_{i}, \lambda_{\max }:=\max _{i} \lambda_{i}$. If

$$
\frac{1}{\sqrt{\lambda_{\min }}}-\frac{1}{\sqrt{\lambda_{\max }}} \leq \frac{\beta_{2}(\bar{x})}{\sqrt{q(\lfloor\bar{x}\rceil)-q(\bar{x})}}
$$

$(C Q I P)$ has the Strong Rounding Property and an optimal solution is given by $\left\lfloor-\frac{1}{2} Q^{-1} L\right\rceil$.

Proof. Analogously to the proof of Lemma 5.9 we get that $\mathcal{L}_{\leq}(z)$ is $\left(\beta_{2}(\bar{x}), 2\right)$-quasi-round w.r.t. $\bar{x}$ for all $q(\bar{x}) \leq z \leq q(\lfloor\bar{x}\rceil)$. Since $\min \{q(x): x \in \operatorname{Round}(\bar{x})\} \leq q(\lfloor\bar{x}\rceil)$ Corollary 2.17 makes sure that $(C Q I P)$ has the SRP.

Applying Theorem 2.24 we get a finite candidate set for any convex quadratic integer problem where $Q$ is positive definite.

Corollary 5.11. Let $\lambda_{1}, \ldots, \lambda_{n}$ denote the eigenvalues of $Q$ and $\lambda_{\min }:=\min _{i} \lambda_{i}, \lambda_{\max }:=$ $\max _{i} \lambda_{i}$. Let $\bar{x}$ be the unique continuous minimizer to $(C Q I P)$, choose $\widetilde{x} \in \operatorname{argmin}\{q(x): x \in$ $\operatorname{Round}(\bar{x})\}$ and let $c:=\min \left\{c \in \mathbb{Z}: \alpha_{2}^{c}(\bar{x}) \geq \sqrt{q(\widetilde{x})-q(\bar{x})} \cdot\left(\frac{1}{\sqrt{\lambda_{\min }}}-\frac{1}{\sqrt{\lambda_{\max }}}\right)\right\}$. Then there exists an optimal solution $x^{*} \in X^{*}$ to $(C Q I P)$ such that $\left\|x^{*}-\bar{x}\right\|_{\infty}<c$. 
As mentioned in Section 2.4 this means that $\left\{x \in \mathbb{Z}^{n}:\|x-\bar{x}\|_{\infty}<c\right\}$ is a finite candidate set for $(C Q I P)$ and $\left|\left\{x \in \mathbb{Z}^{n}:\|x-\bar{x}\|_{\infty}<c\right\}\right| \leq(2 c)^{n}$.

Proof. A direct consequence of Lemma 5.8 is that the ellipsoid $E(Q, \bar{x})$ is $(\gamma, 2)$-quasi-round w.r.t. $\bar{x}$ for $\gamma:=\frac{1}{\sqrt{\lambda_{\min }}}-\frac{1}{\sqrt{\lambda_{\max }}}$.

This means that any level set $\mathcal{L}_{\leq}(z)=\sqrt{z-q(\bar{x})} E(Q, \bar{x})$ is $(\sqrt{z-q(\bar{x})} \gamma, 2)$-quasi-round w.r.t. $\bar{x}$ and hence if $\sqrt{q(\widetilde{x})-q(\bar{x})} \gamma \leq \alpha_{2}^{c}(\bar{x})$ we have that $\mathcal{L}_{\leq}(z)$ is $\left(\alpha_{2}^{c}(\bar{x}), 2\right)$-quasi-round w.r.t. $\bar{x}$ for all $z \leq q(\widetilde{x})$. Since $\min \left\{q(x): x \in \mathbb{Z}^{n},\|x-\bar{x}\|_{\infty}<c\right\} \leq q(\widetilde{x})$ for all $c \in \mathbb{Z}$ this means that the assumptions of Theorem 2.24 are fulfilled and hence the claim.

For the special case of convex quadratic functions as considered here we adapt the notation of $\epsilon$-similar sets as given in Definition 3.43 to derive the definition of $\alpha$-quasi-axisparallel sets.

Definition 5.12. Let $\alpha \geq 0$. A set $M \subseteq \mathbb{R}^{n}$ is called $\alpha$-quasi-axisparallel w.r.t. $x^{0}$ if there exist $t \geq 0, r, R \in \mathbb{R}_{0}^{+}$such that

$$
r \cdot E\left(\operatorname{Diag}(t), x^{0}\right) \subseteq M \subseteq R \cdot E\left(\operatorname{Diag}(t), x^{0}\right) \quad \text { and } R-r \leq \alpha .
$$

Remark 5.13. - For any $\alpha$-quasi-axisparallel set $M$ there exists $t \geq 0$ such that $M$ is $\alpha$-similar to the set $E\left(\operatorname{Diag}(t), x^{0}\right)$ and hence any $\alpha$-quasi-axisparallel set is $\alpha$-quasicross-shaped, compare Definition 3.43.

- $(\alpha, 2)$-quasi-round sets are $\alpha$-quasi-axisparallel with $t=(1, \ldots, 1)$, compare Definition 2.1.

- If $\alpha=0$ Definition 5.12 means that $M$ has to be an axisparallel ellipsoid centered at $x^{0}$.

- If a set $M$ is $\alpha$-quasi-axisparallel w.r.t. $x^{0}$ it is also $\widetilde{\alpha}$-quasi-axisparallel w.r.t. $x^{0}$ for all $\widetilde{\alpha} \geq \alpha$.

- We use the notation that $M$ is $\alpha(t)$-quasi-axisparallel w.r.t. $x^{0}$ in the following. By this notation we mean that there exists $\hat{t} \geq 0, r, R \in \mathbb{R}_{0}^{+}$s.t. $r \cdot E\left(\operatorname{Diag}(\hat{t}), x^{0}\right) \subseteq M \subseteq$ $R \cdot E\left(\operatorname{Diag}(\hat{t}), x^{0}\right)$ and $R-r \leq \alpha(\hat{t})$.

- Note here that we allow in this general definition $t \geq 0$, i.e., $E\left(\operatorname{Diag}(t), x^{0}\right)$ unbounded. This is of course only possible for unbounded sets $M$.

An example for a quasi-axisparallel ellipsoid is given in Figure 5.5.

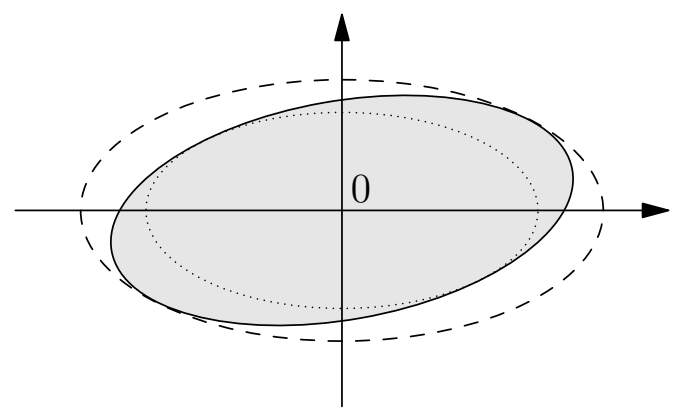

Figure 5.5.: A quasi-axisparallel ellipsoid. 
Corollary 5.14. Let $\bar{x}$ be the unique continuous minimizer to $(C Q I P)$ and choose $\widetilde{x} \in$ $\operatorname{argmin}\{q(x): x \in \operatorname{Round}(\bar{x})\}$. If $E(Q, \bar{x})$ is $\frac{\alpha(t, \bar{x})}{\sqrt{q(\widetilde{x})-q(\bar{x})}}$-quasi-axisparallel w.r.t. $\bar{x}$, where

$$
\alpha(t, \bar{x}):=\min _{y \in \mathbb{Z}^{n} \backslash \operatorname{Round}(\bar{x})}\|y-\bar{x}\|_{t}-\min _{y \in \mathbb{Z}^{n}}\|y-\bar{x}\|_{t},
$$

(CQIP) has the Rounding Property.

Here we use the notation $\|x\|_{t}:=\sqrt{x^{t} \operatorname{Diag}(t) x}$ for $t \geq 0$.

Proof. As in the proof of Lemma 5.9 we get that if $E(Q, \bar{x})$ is $\frac{\alpha(t, \bar{x})}{\sqrt{q(\widetilde{x})-q(\bar{x})}}$-quasi-axisparallel w.r.t. $\bar{x}, \mathcal{L}_{\leq, q}(z)$ is $\alpha(t, \bar{x})$-quasi-axisparallel w.r.t. $\bar{x}$ for all levels $q(\bar{x}) \leq z \leq q(\widetilde{x})=$ $\min \{q(x): x \in \operatorname{Round}(\bar{x})\}$. Hence the claim follows with Theorem 3.45 and the observation that $p_{E(\operatorname{Diag}(t), \bar{x})}(x)=\|x-\bar{x}\|_{t}$.

As with quasi-roundness and as mentioned in Section 3.5 the challenge is to determine $\alpha(t, \bar{x})$.

Lemma 5.15. For given $x, t \in \mathbb{R}^{n}, t \geq 0$ let $a_{j}=\left|x_{j}-\left\lfloor x_{j}\right\rceil\right|$ and choose $l \in \underset{j=1, \ldots, n}{\operatorname{argmin}}\left\{\left(2 a_{j}+\right.\right.$ 1) $\left.t_{j}\right\}$. Then we have

$$
\alpha(t, x)=\sqrt{t_{l}\left(1+a_{l}\right)^{2}+\sum_{j \neq l} t_{j} a_{j}^{2}}-\sqrt{\sum_{j=1}^{n} t_{j} a_{j}^{2}} .
$$

Proof. (i) Using Lemma 5.6 we get that $\min _{y \in \mathbb{Z}^{n}}\|y-x\|_{t}=\|\lfloor x\rceil-x\|_{t}$ for all $t \geq 0$.

(ii) Define $\widetilde{x}$ by

$$
\widetilde{x}_{j}= \begin{cases}\left\lceil x_{l}\right\rceil+1 & \text { if } j=l \text { and }\left\lfloor x_{l}\right\rceil=\left\lceil x_{l}\right\rceil \\ \left\lfloor x_{l}\right\rfloor-1 & \text { if } j=l \text { and }\left\lfloor x_{l}\right\rceil=\left\lfloor x_{l}\right\rfloor \neq x_{l} \\ \left\lfloor x_{j}\right\rceil & \text { if } j \neq l\end{cases}
$$

This means, $\left|\widetilde{x}_{j}-x_{j}\right|=a_{j}$ for all $j \neq l$ and $\left|\widetilde{x}_{l}-x_{l}\right|=1+a_{l}$.

We show that $\widetilde{x} \in \operatorname{argmin}_{y \in \mathbb{Z}^{n} \backslash \operatorname{Round}(x)}\|y-x\|_{t}$ :

$\widetilde{x} \in \mathbb{Z}^{n}$ but $\widetilde{x} \notin \operatorname{Round}(x)$ as for all $y \in \operatorname{Round}(x)$ and for all $j$ we have $\left|x_{j}-y_{j}\right|<1$, but $\left|\widetilde{x}_{l}-x_{l}\right|=1+a_{l} \geq 1$, as $a_{l} \geq 0$.

Let $y \in \mathbb{Z}^{n} \backslash \operatorname{Round}(x)$ : this means, there exists $k \in\{1, \ldots, n\}$ such that $\left|x_{k}-y_{k}\right| \geq$ 1 and hence $\left|x_{k}-y_{k}\right| \geq 1+a_{k}$ as $y_{k} \in \mathbb{Z}$. Furthermore, we have $\left|x_{j}-y_{j}\right| \geq a_{j}$ as $y_{j} \in \mathbb{Z}$.

Case 1: if $k=l$ we have

$$
\begin{aligned}
\|x-\widetilde{x}\|_{t}^{2}-\|x-y\|_{t}^{2} & =\sum_{j \neq l} \underbrace{t_{j}}_{\geq 0}[a_{j}^{2}-\underbrace{\left(x_{j}-y_{j}\right)^{2}}_{\geq a_{j}^{2}}]+t_{l}\left[\left(x_{l}-\widetilde{x}_{l}\right)^{2}-\left(x_{l}-y_{l}\right)^{2}\right] \\
& \leq t_{l}[\underbrace{\left(x_{l}-\widetilde{x}_{l}\right)^{2}}_{=\left(1+a_{l}\right)^{2}}-\underbrace{\left(x_{l}-y_{l}\right)^{2}}_{\geq\left(1+a_{l}\right)^{2}}] \leq 0 .
\end{aligned}
$$


Case 2: if $k \neq l$ we have $\left|x_{k}-y_{k}\right| \geq 1+a_{k},\left|x_{l}-y_{l}\right| \geq a_{l}$ and $\left|x_{k}-\widetilde{x}_{k}\right|=a_{k}$, $\left|x_{l}-\widetilde{x}_{l}\right|=1+a_{l}$. Hence we get

$$
\begin{aligned}
\|x-\widetilde{x}\|_{t}^{2}-\|x-y\|_{t}^{2} & \leq t_{l}\left[\left(x_{l}-\widetilde{x}_{l}\right)^{2}-\left(x_{l}-y_{l}\right)^{2}\right]+t_{k}\left[\left(x_{k}-\widetilde{x}_{k}\right)^{2}-\left(x_{k}-y_{k}\right)^{2}\right] \\
& \leq t_{l}\left[\left(1+a_{l}\right)^{2}-a_{l}^{2}\right]-t_{k}\left[\left(1+a_{k}\right)^{2}-a_{k}^{2}\right] \leq 0
\end{aligned}
$$

as $t_{l}\left(2 a_{l}+1\right) \leq t_{k}\left(2 a_{k}+1\right)$.

Together we get

$$
\alpha(t, x)=\|x-\widetilde{x}\|_{t}-\|\lfloor x\rceil-x\|_{t}=\sqrt{t_{l}\left(1+a_{l}\right)^{2}+\sum_{j \neq l} t_{j} a_{j}^{2}}-\sqrt{\sum_{j=1}^{n} t_{j} a_{j}^{2}} .
$$

We can adapt the assumptions of Corollary 5.14 to even get the Strong Rounding Property.

Lemma 5.16. Let $\bar{x}$ be the unique continuous minimizer to $(C Q I P)$. If $E(Q, \bar{x})$ is $\frac{\beta(t, \bar{x})}{\sqrt{q(\lfloor\bar{x}\rceil)-q(\bar{x})}}$-quasi-axisparallel w.r.t. $\bar{x}$, where

$$
\beta(t, \bar{x}):=\min _{y \in \mathbb{Z}^{n} \backslash\{\lfloor\bar{x}\rceil\}}\|y-\bar{x}\|_{t}-\min _{y \in \mathbb{Z}^{n}}\|y-\bar{x}\|_{t},
$$

$(C Q I P)$ has the Strong Rounding Property.

Proof. Since $\beta(t, \bar{x}) \leq \alpha(t, \bar{x})$ we have that $(C Q I P)$ has the RP, compare Corollary 5.14. This means that $\widetilde{x} \in \operatorname{argmin}\{q(x): x \in \operatorname{Round}(\bar{x})\}$ is an optimal solution to $(C Q I P)$.

Assume that $q(\widetilde{x})<q(\lfloor\bar{x}\rceil)$. This means, first of all $\widetilde{x} \neq\lfloor\bar{x}\rceil$. Furthermore, we know that $\mathcal{L}_{\leq, q}(q(\widetilde{x}))=\sqrt{q(\widetilde{x})-q(\bar{x})} E(Q, \bar{x})$ is $\beta(t, \bar{x})$-quasi-axisparallel w.r.t. $\bar{x}$, i.e., there exist $t \geq 0$ $r, R \in \mathbb{R}_{0}^{+}$such that

$$
r \cdot E(\operatorname{Diag}(t), \bar{x}) \subseteq \mathcal{L}_{\leq, q}(q(\widetilde{x})) \subseteq R \cdot E(\operatorname{Diag}(t), \bar{x}) \quad \text { and } R-r \leq \beta(t, \bar{x}) .
$$

Since $\min _{y \in \mathbb{Z}^{n}}\|y-x\|_{t}=\|\lfloor x\rceil-x\|_{t}$ for all $t \geq 0$ we get

$$
\begin{aligned}
\|\lfloor\bar{x}\rceil-\bar{x}\|_{t} & =\min _{y \in \mathbb{Z}^{n} \backslash\{\lfloor\bar{x}\rceil\}}\|y-\bar{x}\|_{t}-\beta(t, \bar{x}) \\
& \leq\|\widetilde{x}-\bar{x}\|_{t}-\beta(t, \bar{x}) \quad \text { as } \widetilde{x} \neq\lfloor\bar{x}\rceil, \widetilde{x} \in \mathbb{Z}^{n} \\
& \leq R-\beta(t, \bar{x}) \quad \text { as } \widetilde{x} \in \mathcal{L}_{\leq, q}(q(\widetilde{x})) \subseteq R \cdot E(\operatorname{Diag}(t), \bar{x}) \\
& \leq r .
\end{aligned}
$$

Hence $\lfloor\bar{x}\rceil \in r \cdot E(\operatorname{Diag}(t), \bar{x}) \subseteq \mathcal{L}_{\leq}(q(\widetilde{x}))$ which is a contradiction to $q(\widetilde{x})<q(\lfloor\bar{x}\rceil)$.

As in Lemma 2.19 we can also determine $\beta(t, x)$.

Lemma 5.17. For given $x, t \in \mathbb{R}^{n}, t \geq 0$ let $a_{j}:=\left|x_{j}-\left\lfloor x_{j}\right\rceil\right|$ and choose $l \in \underset{j=1, \ldots, n}{\operatorname{argmax}}\left\{\left(2 a_{j}-\right.\right.$ 1) $\left.t_{j}\right\}$. Then we have

$$
\beta(t, x)=\sqrt{t_{l}\left(1-a_{l}\right)^{2}+\sum_{j \neq l} t_{j} a_{j}^{2}}-\sqrt{\sum_{j=1}^{n} t_{j} a_{j}^{2}} .
$$


Proof. Define $\widetilde{x} \in \mathbb{Z}^{n}$ by

$$
\widetilde{x}_{j}=\left\{\begin{array}{ll}
\left\lfloor x_{j}\right\rceil & \text { if } j \neq l \\
\left\lceil x_{l}\right\rceil & \text { if } j=l,\left\lfloor x_{l}\right\rceil=\left\lfloor x_{l}\right\rfloor \neq x_{l} \\
\left\lfloor x_{l}\right\rfloor & \text { if } j=l,\left\lfloor x_{l}\right\rceil=\left\lceil x_{l}\right\rceil \neq x_{l} \\
x_{l}+1 & \text { if } j=l, x_{l} \in \mathbb{Z}
\end{array} .\right.
$$

This means, $\left|\widetilde{x}_{l}-x_{l}\right|=1-a_{l}$ and $\left|\widetilde{x}_{j}-x_{j}\right|=a_{j}$ for all $j \neq l$.

We show that $\widetilde{x} \in \operatorname{argmin}_{y \in \mathbb{Z}^{n} \backslash\{\lfloor x\rceil\}}\|x-y\|_{t}$ : Obviously $\widetilde{x} \neq\lfloor x\rceil$ since $\widetilde{x}_{l} \neq\left\lfloor x_{l}\right\rceil$. It remains to be shown that $\|\widetilde{x}-x\|_{t} \leq\|y-x\|_{t}$ for all $y \in \mathbb{Z}^{n} \backslash\{\lfloor x\rceil\}$ :

Let $y \in \mathbb{Z}^{n} \backslash\{\lfloor x\rceil\}$. Then there is a $k \in\{1, \ldots, n\}$ with $y_{k} \neq\left\lfloor x_{k}\right\rceil$.

(i) If $k=l$ we have

$$
\begin{aligned}
& \|x-\widetilde{x}\|_{t}^{2}-\|x-y\|_{t}^{2}=\sum_{j \neq l} \underbrace{t_{j}}_{\geq 0}[a_{j}^{2}-\underbrace{\left(x_{j}-y_{j}\right)^{2}}_{\geq a_{j}^{2}}]+t_{l}\left[\left(x_{l}-\widetilde{x}_{l}\right)^{2}-\left(x_{l}-y_{l}\right)^{2}\right] \\
\leq & \underbrace{t_{l}}_{\geq 0}\left[\left(x_{l}-\widetilde{x}_{l}\right)^{2}-\left(x_{l}-y_{l}\right)^{2}\right] \leq 0 \quad \text { as } y_{l} \in \mathbb{Z} \backslash\left\{\left\lfloor x_{l}\right\rceil\right\} .
\end{aligned}
$$

Hence $\|x-\widetilde{x}\|_{t} \leq\|x-y\|_{t}$.

(ii) If $k \neq l$ we have $\left|x_{k}-y_{k}\right| \geq 1-a_{k}$ and $\left|x_{l}-y_{l}\right| \geq a_{l}$. Therefore

$$
\begin{aligned}
\|x-\widetilde{x}\|_{t}^{2}-\|x-y\|_{t}^{2} & \leq \underbrace{t_{l}}_{\geq 0}\left[\left(x_{l}-\widetilde{x}_{l}\right)^{2}-\left(x_{l}-y_{l}\right)^{2}\right]+\underbrace{t_{k}}_{\geq 0}\left[\left(x_{k}-\widetilde{x}_{k}\right)^{2}-\left(x_{k}-y_{k}\right)^{2}\right] \\
& \leq t_{l}\left[\left(1-a_{l}\right)^{2}-a_{l}^{2}\right]-t_{k}\left[\left(1-a_{k}\right)^{2}-a_{k}^{2}\right] \leq 0
\end{aligned}
$$

as $t_{l}\left(1-2 a_{l}\right) \leq t_{k}\left(1-2 a_{k}\right)$. And accordingly $\|x-\widetilde{x}\|_{t} \leq\|x-y\|_{t}$.

This means,

$$
\beta(t, x)=\|\widetilde{x}-x\|_{t}-\|\lfloor x\rceil-x\|_{t}=\sqrt{t_{l}\left(1-a_{l}\right)^{2}+\sum_{j \neq l} t_{j} a_{j}^{2}}-\sqrt{\sum_{j=1}^{n} t_{j} a_{j}^{2}} .
$$

We point out here that for any $t \geq 0$ the second closest integer point $\widetilde{x}$ to $x$ with respect to $\|\cdot\|_{t}$, we used to determine $\beta(t, x)$, is always in $\operatorname{Round}(x) \backslash\{\lfloor x\rceil\}$ if $x \notin \mathbb{Z}^{n}$.

As mentioned before the class of $\alpha(t, \bar{x})$ - (or $\beta(t, \bar{x})$ - respectively) quasi-axisparallel ellipsoids contains both the class of axisparallel and the class of $\left(\alpha_{2}(\bar{x}), 2\right)$ - (or $\left(\beta_{2}(\bar{x}), 2\right)$ - respectively) quasi-round ellipsoids as subclasses. This means that Lemma 5.9 follows from Corollary 5.14 and Lemma 5.6 and Corollary 5.10 follow from Lemma 5.16. But still $\beta(t, \bar{x})-$ quasi-axisparallelity is not necessary for the (Strong) Rounding Property. Compare the example in Figure 5.6, where the level sets of a convex quadratic function $q$ are shown. It is obvious that the corresponding $(C Q I P)$ has the Strong Rounding Property since $\lfloor\bar{x}\rceil \in X^{*}$ but the level sets are not $\beta(t, \bar{x})$-quasi-axisparallel since $\bar{x}=(0.5,0.5)$ and therefore $\beta(t, \bar{x})=0$ for all $t \geq 0$. 


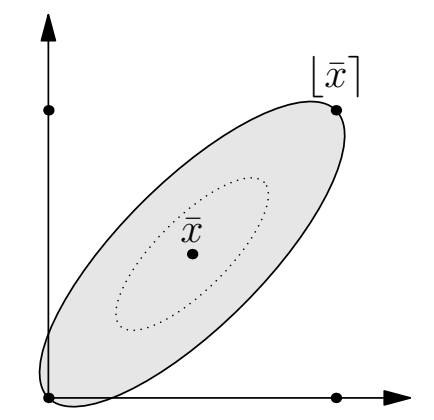

Figure 5.6.: An example for a level set that is not $\beta(t, \bar{x})$-quasi-axisparallel but still guarantees the Strong Rounding Property. 


\subsection{Lower bounds for (CQIP)}

If we consider an arbitrary convex quadratic integer problem it is rather unlikely that the problem has the Rounding Property. Furthermore, if the dimension gets too large the Rounding Property is not too helpful. This raises the question: is there any chance to use some of our ideas also in the case that the given $(C Q I P)$ does not have the Strong Rounding Property? We follow the approach in Buchheim et al. [2012a] and solve such problems by a branchand-bound algorithm. In this section we investigate different ways of getting tight, easy to calculate lower bounds on $\min _{x \in \mathbb{Z}^{n}} q(x)$ by using the following idea

$$
q^{\prime} \text { is a global underestimator of } q \quad \Leftrightarrow \quad \mathcal{L}_{\leq, q^{\prime}}(z) \supseteq \mathcal{L}_{\leq, q}(z) \quad \forall z \in \mathbb{R} .
$$

Since we know that the level sets of $q$ are ellipsoids this motivates the following class of underestimators:

Let $\bar{x}$ be the continuous minimizer of $q$ and let $Q^{\prime}$ be a positive semidefinite matrix such that $E(Q, 0) \subseteq E\left(Q^{\prime}, 0\right)$. Then we consider the underestimator

$$
q^{\prime}(x):=q(\bar{x})+(x-\bar{x})^{t} Q^{\prime}(x-\bar{x}) .
$$

Remark 5.18. (i) These are really underestimators since the level sets of $q^{\prime}$ are of the form $\mathcal{L}_{\leq, q^{\prime}}(z)=\sqrt{z-q(\bar{x})} E\left(Q^{\prime}, \bar{x}\right)$ for all $z \geq q(\bar{x})$ and therefore for $z \neq q(\bar{x}): \mathcal{L}_{\leq, q^{\prime}}(z) \supseteq$ $\mathcal{L}_{\leq, q}(z) \Leftrightarrow E(Q, 0) \subseteq E\left(Q^{\prime}, 0\right)$.

(ii) We allow here positive semidefinite $Q^{\prime}$, in contrast to $(C Q I P)$ where we assumed that $Q$ is positive definite.

(iii) Remember also that $E(Q, 0) \subseteq E\left(Q^{\prime}, 0\right)$ is equivalent to $Q-Q^{\prime} \succeq 0$, compare again Remark 5.3.

Since $q^{\prime}$ is a global underestimator of $q$ we know that $\min \left\{q^{\prime}(x): x \in \mathbb{Z}^{n}\right\} \leq \min \{q(x): x \in$ $\left.\mathbb{Z}^{n}\right\}$. We denote by $x_{q}^{*}$ an optimal solution to $\min \left\{q(x): x \in \mathbb{Z}^{n}\right\}$ and by $x_{q^{\prime}}^{*}$ an optimal solution to $\min \left\{q^{\prime}(x): x \in \mathbb{Z}^{n}\right\}$.

Geometrically speaking, the idea (first proposed by Buchheim et al. [2012a]) is the following: we surround $E(Q, \bar{x})$ by another ellipsoid $E\left(Q^{\prime}, \bar{x}\right)$. Then we know that if we scale these two ellipsoids simultaneously such that $r \cdot E\left(Q^{\prime}, \bar{x}\right)$ contains no integer point in its interior and at least one on its boundary, also $r \cdot E(Q, \bar{x})$ contains no integer in its interior. Of course we know how to scale $E\left(Q^{\prime}, \bar{x}\right): \sqrt{q^{\prime}\left(x_{q^{\prime}}^{*}\right)-q(\bar{x})} E\left(Q^{\prime}, \bar{x}\right)$ contains $x_{q^{\prime}}^{*}$ on its boundary and no integer point in its interior. This means, we get the lower bound $l b:=q^{\prime}\left(x_{q^{\prime}}^{*}\right) \leq \min _{x \in \mathbb{Z}^{n}} q(x)$. See Figure 5.7 for an illustration: the dark gray ellipsoid is $\sqrt{q^{\prime}\left(x_{q^{\prime}}^{*}\right)-q(\bar{x})} E(Q, \bar{x})$ whereas the light gray ellipsoid is $\sqrt{q^{\prime}\left(x_{q^{\prime}}^{*}\right)-q(\bar{x})} E\left(Q^{\prime}, \bar{x}\right)$ and the dotted ellipsoid is $\sqrt{q\left(x_{q}^{*}\right)-q(\bar{x})} E(Q, \bar{x})$ for comparison, i.e., the level set of $q$ corresponding to the optimal objective value of $(C Q I P)$.

This approach is of course only helpful if it is significantly easier to calculate $\min \left\{q^{\prime}(x): x \in\right.$ $\left.Z^{n}\right\}$ than $\min \left\{q(x): x \in \mathbb{Z}^{n}\right\}$. To guarantee this we use only such underestimators $q^{\prime}$ that have the Strong Rounding Property. Among these "easy to calculate" lower bounds we are 


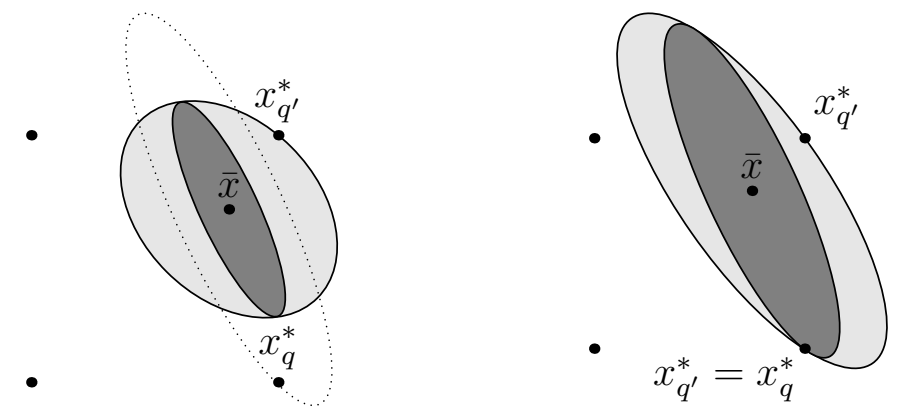

Figure 5.7.: Illustration of the lower bound calculation: in the left picture we get $l b<$ $\min _{x \in \mathbb{Z}^{2}} q(x)$ whereas in the right picture we get $l b=\min _{x \in \mathbb{Z}^{2}} q(x)$.

looking for the tightest lower bound. Hence we would like to solve the following optimization problem

$$
\begin{array}{cl}
\max & (\lfloor\bar{x}\rceil-\bar{x})^{t} Q^{\prime}(\lfloor\bar{x}\rceil-\bar{x}) \\
\text { s.t. } & Q-Q^{\prime} \succeq 0 \\
& Q^{\prime} \succeq 0 \\
& q^{\prime}(x)=q(\bar{x})+(x-\bar{x})^{t} Q^{\prime}(x-\bar{x}) \text { has the SRP : }
\end{array}
$$

the constraint $Q-Q^{\prime} \succeq 0$ makes sure that $q^{\prime}$ is indeed an underestimator of $q$, as mentioned before. The requirement that $\left(q^{\prime}, \mathbb{R}^{n}\right)$ has the Strong Rounding Property makes sure that the lower bound is easy to calculate and we would like to maximize the lower bound which is given by $\min \left\{q^{\prime}(x): x \in \mathbb{Z}^{n}\right\}=q^{\prime}(\lfloor\bar{x}\rceil)=q(\bar{x})+(\lfloor\bar{x}\rceil-\bar{x})^{t} Q^{\prime}(\lfloor\bar{x}\rceil-\bar{x})$ if $q^{\prime}$ has the Strong Rounding Property.

A first result about problem (5.2) is stated in the next lemma.

Lemma 5.19. Let $Q^{\prime}$ be feasible for (5.2). If $\left\{x: x^{t} Q x=1\right\} \cap\left\{x: x^{t} Q^{\prime} x=1\right\}=\emptyset, Q^{\prime}$ is not an optimal solution to (5.2).

Proof. Since $\left\{x: x^{t} Q x=1, x^{t} Q^{\prime} x=1\right\}=\emptyset$ and $Q-Q^{\prime} \succeq 0$ we have $x^{t} Q x>x^{t} Q^{\prime} x$ for all $x \in \mathbb{R}^{n}:$ if $\hat{x}^{t} Q \hat{x}=\hat{x}^{t} Q^{\prime} \hat{x}$ for some $\hat{x} \in \mathbb{R}^{n}, \frac{1}{\sqrt{\hat{x}^{t} Q \hat{x}}} \hat{x} \in\left\{x: x^{t} Q x=1, x^{t} Q^{\prime} x=1\right\}$.

If $(\lfloor\bar{x}\rceil-\bar{x})^{t} Q^{\prime}(\lfloor\bar{x}\rceil-\bar{x}) \neq 0$ choose $\lambda:=\min _{x \in \mathbb{R}^{n}} \frac{x^{t} Q x}{x^{t} Q^{\prime} x}$ and define $Q^{\prime \prime}:=\lambda Q^{\prime}$. Then we have $Q-Q^{\prime \prime} \succeq 0$ since $x^{t}\left(Q-\lambda Q^{\prime}\right) x \geq 0$ by definition of $\lambda, Q^{\prime \prime} \succeq 0$ since $\lambda \geq 0$ and $q^{\prime \prime}$ has the SRP since $\bar{x}_{q^{\prime \prime}}=\bar{x}_{q^{\prime}}$ and $x_{q^{\prime \prime}}^{*}=x_{q^{\prime}}^{*}$ and we assumed $q^{\prime}$ to have the SRP. Hence $Q^{\prime \prime}$ is feasible for (5.2). Furthermore, we have $(\lfloor\bar{x}\rceil-\bar{x})^{t} Q^{\prime \prime}(\lfloor\bar{x}\rceil-\bar{x})=\lambda(\lfloor\bar{x}\rceil-\bar{x})^{t} Q^{\prime}(\lfloor\bar{x}\rceil-\bar{x})>(\lfloor\bar{x}\rceil-\bar{x})^{t} Q^{\prime}(\lfloor\bar{x}\rceil-\bar{x})$ since $(\lfloor\bar{x}\rceil-\bar{x})^{t} Q^{\prime}(\lfloor\bar{x}\rceil-\bar{x}) \neq 0$ and $\lambda>1$.

If $(\lfloor\bar{x}\rceil-\bar{x})^{t} Q^{\prime}(\lfloor\bar{x}\rceil-\bar{x})=0$ choose $Q^{\prime \prime}=\lambda_{\min } I_{n}$ where $\lambda_{i}$ are the eigenvalues of $Q$ and $\lambda_{\min }:=\min _{i=1, \ldots, n} \lambda_{i}$. Then we have $Q-Q^{\prime \prime} \succeq 0, Q^{\prime \prime} \succeq 0$ and $q^{\prime \prime}(x)$ is separable convex. Hence $Q^{\prime \prime}$ is feasible for (5.2) and we have $(\lfloor\bar{x}\rceil-\bar{x})^{t} Q^{\prime \prime}(\lfloor\bar{x}\rceil-\bar{x})=\lambda_{\min } \sum_{i=1}^{n}\left(\left\lfloor\bar{x}_{i}\right\rceil-\bar{x}_{i}\right)^{2}>0$ since we always assume $\bar{x} \notin \mathbb{Z}^{n}$.

Geometrically this means that we choose $Q^{\prime}$ such that it "touches" $Q$. 
We are not able to solve problem (5.2) directly as we cannot handle the constraint " $q$ ' $(x)$ has the SRP". Therefore we consider special cases of problem (5.2) where we replace this constraint by a stronger one that is sufficient but not necessary for the Strong Rounding Property, like for example $Q^{\prime}=\operatorname{Diag}(t)$ for some $t \geq 0$. Hence we do not find the tightest lower bound in general, but as any matrix $Q^{\prime} \succeq 0$ such that $Q-Q^{\prime} \succeq 0$ provides us with a lower bound we will always find a lower bound. This means also that even a heuristic solution to (5.2) (or a problem with a stronger constraint), gives us a lower bound. We use this fact later on.

First we find the best axisparallel ellipsoid $E\left(Q^{\prime}, \bar{x}\right)$ that provides us with an underestimator $q^{\prime}$ of $q$.

Lemma 5.20. The tightest lower bound we can obtain by underestimating $q$ by $q^{\prime}(x):=$ $q(\bar{x})+(x-\bar{x})^{t} Q^{\prime}(x-\bar{x})$ with axisparallel level sets $\sqrt{z-q(\bar{x})} E\left(Q^{\prime}, \bar{x}\right)$ is given by the optimal objective value of the problem

$$
\begin{array}{ll}
\max & \sum_{i=1}^{n} t_{i}\left(\left\lfloor\bar{x}_{i}\right\rceil-\bar{x}_{i}\right)^{2} \\
\text { s.t. } & Q-\operatorname{Diag}(t) \succeq 0 \\
& t \geq 0 .
\end{array}
$$

An optimal solution $t^{*}$ defines the underestimator $q^{\prime}(x)=q(\bar{x})+\sum_{i=1}^{n} t_{i}^{*}\left(x_{i}-\bar{x}_{i}\right)^{2}$ and gives the bound $q(\bar{x})+\sum_{i=1}^{n} t_{i}^{*}\left(\left\lfloor\bar{x}_{i}\right\rceil-\bar{x}_{i}\right)^{2}$.

Proof. To derive this program we recall that $E\left(Q^{\prime}, 0\right)$ is axisparallel if and only if $Q^{\prime}=\operatorname{Diag}(t)$ for some $t \geq 0$, compare Lemma 5.4. Then we use $Q^{\prime}=\operatorname{Diag}(t)$ to simplify the objective function.

This problem has already been stated in Buchheim et al. [2012a] and can be solved efficiently as it is a semidefinite program.

If we use not axisparallelity but quasi-roundness to ensure the Strong Rounding Property of $q^{\prime}$, we observe first of all that this means that $Q^{\prime}$ has to be positive definite since only bounded sets can be quasi-round. Recall that $E\left(Q^{\prime}, \bar{x}\right)$ has to be $\left(\frac{\beta_{2}(\bar{x})}{\sqrt{q^{\prime}(\lfloor\bar{x}])-q(\bar{x})}}, 2\right)$-quasi-round to ensure the Strong Rounding Property.

Lemma 5.21. The tightest lower bound we can obtain by underestimating $q$ by $q^{\prime}(x):=$ $q(\bar{x})+(x-\bar{x})^{t} Q^{\prime}(x-\bar{x})$ with $\left(\beta_{2}(\bar{x}), 2\right)$-quasi-round level sets $\sqrt{z-q(\bar{x})} E\left(Q^{\prime}, \bar{x}\right)$ for all levels $q(\bar{x}) \leq z \leq q^{\prime}(\lfloor\bar{x}\rceil)$ is given by the optimal objective value of the problem

$$
\begin{array}{cl}
\max & (\lfloor\bar{x}\rceil-\bar{x})^{t} Q^{\prime}(\lfloor\bar{x}\rceil-\bar{x}) \\
\text { s.t. } & Q-Q^{\prime} \succeq 0 \\
& R^{2} Q^{\prime}-I_{n} \succeq 0 \\
& I_{n}-r^{2} Q^{\prime} \succeq 0 \\
& R-r \leq \frac{\beta_{2}(\bar{x})}{\sqrt{q^{\prime}(\lfloor\bar{x}\rceil)-q(\bar{x})}} \\
& r, R \geq 0 .
\end{array}
$$

An optimal solution $Q_{*}^{\prime}$ defines the underestimator $q^{\prime}(x)=q(\bar{x})+(x-\bar{x})^{t} Q_{*}^{\prime}(x-\bar{x})$ and gives the bound $q(\bar{x})+(\lfloor\bar{x}\rceil-\bar{x})^{t} Q_{*}^{\prime}(\lfloor\bar{x}\rceil-\bar{x})$. 
Proof. As mentioned before $\sqrt{z-q(\bar{x})} E\left(Q^{\prime}, \bar{x}\right)$ is $\left(\beta_{2}(\bar{x}), 2\right)$-quasi-round w.r.t. $\bar{x}$ for all $q(\bar{x}) \leq$ $z \leq q^{\prime}(\lfloor\bar{x}\rceil)$, if $E\left(Q^{\prime}, \bar{x}\right)$ is $\left(\frac{\beta_{2}(\bar{x})}{\sqrt{q^{\prime}(\lfloor\bar{x}\rceil)-q(\bar{x})}}, 2\right)$-quasi-round w.r.t. $\bar{x}$. This is if $B_{2}(\bar{x}, r) \subseteq$ $E\left(Q^{\prime}, \bar{x}\right) \subseteq B_{2}(\bar{x}, R)$ and $R-r \leq \frac{\beta_{2}(\bar{x})}{\sqrt{q^{\prime}(\lfloor\bar{x}\rceil)-q(\bar{x})}}$ or equivalently $I_{n}-r^{2} Q^{\prime} \succeq 0, R^{2} Q^{\prime}-I_{n} \succeq 0$ and $R-r \leq \frac{\beta_{2}(\bar{x})}{\sqrt{q^{\prime}(\lfloor\bar{x}\rceil)-q(\bar{x})}}$.

The variables in (5.4) are $Q^{\prime}, r$ and $R$, meaning that the second, third and fourth constraint are non-linear. This means that problem (5.4) is not an SDP and so far it is not known how to solve this problem. Therefore we use the following heuristic: we fix the eigenvectors of $Q^{\prime}$ to be the same as the eigenvectors of $Q$ and adapt the eigenvalues to make sure that $Q^{\prime}$ is quasi-round. By only reducing the eigenvalues we make sure that $Q-Q^{\prime} \succeq 0$. An important property of this heuristic is that if $Q$ is already quasi-round, the heuristic gives $Q^{\prime}=Q$, which yields of course the tightest lower bound.

Lemma 5.22. Assume that $(C Q I P)$ does not have the Strong Rounding Property. Let $Q=V^{t} \operatorname{Diag}(\lambda) V$ be the eigendecompositon of $Q$ and let $\lambda_{\min }:=\min _{i} \lambda_{i}$. Define $Q^{\prime}:=$ $V^{t} \operatorname{Diag}\left(\lambda^{\prime}\right) V$ where

$$
\lambda_{i}^{\prime}=\min \left\{\lambda_{i}, \frac{\lambda_{\min }}{\left(1-\frac{\beta_{2}(\bar{x})}{\sqrt{q(\lfloor\bar{x}\rceil)-q(\bar{x})}} \sqrt{\lambda_{\min }}\right)^{2}}\right\} \quad \forall i=1, \ldots, n .
$$

Then we have that $Q^{\prime}$ is an optimal solution to the following problem

$$
\begin{array}{cl}
\max & (\lfloor\bar{x}\rceil-\bar{x})^{t} Q^{\prime}(\lfloor\bar{x}\rceil-\bar{x}) \\
\text { s.t. } & Q-Q^{\prime} \succeq 0 \\
& Q^{\prime} \text { has the same eigenvectors as } Q \\
& R^{2} Q^{\prime}-I_{n} \succeq 0 \\
& I_{n}-r^{2} Q^{\prime} \succeq 0 \\
& R-r \leq \frac{\beta_{2}(\bar{x})}{\sqrt{q(\lfloor\bar{x}\rceil)-q(\bar{x})}} \\
& r, R \geq 0 .
\end{array}
$$

Proof. As $\left(q, \mathbb{R}^{n}\right)$ does not have the SRP we know that $\sqrt{q(\lfloor\bar{x}\rceil)-q(\bar{x})} E(Q, \bar{x})$ is not $\left(\beta_{2}(\bar{x}), 2\right)$-quasi-round, i.e., $\frac{1}{\sqrt{\lambda_{\min }}}>\frac{\beta_{2}(\bar{x})}{\sqrt{q(\lfloor\bar{x}\rceil)-q(\bar{x})}}$. This means that $\lambda_{i}^{\prime}$ is well-defined for all $i=1, \ldots, n$.

- We show first that $Q^{\prime}$ is feasible for (5.5): The eigenvalues of $Q^{\prime}$ are $\lambda_{i}^{\prime} \geq 0$, the eigenvalues of $Q-Q^{\prime}$ are $\lambda_{i}-\lambda_{i}^{\prime} \geq 0$, since $Q$ and $Q^{\prime}$ are simultaneously diagonalizable and therefore $Q-Q^{\prime} \succeq 0$. By construction we also have that $Q^{\prime}$ and $Q$ have the same eigenvectors. Since we know that $0<\lambda_{i}^{\prime} \leq \frac{\lambda_{\min }}{\left(1-\frac{\beta_{2}(\bar{x})}{\sqrt{q(\lfloor\bar{x}\rceil)-q(\bar{x})}} \sqrt{\lambda_{\min }}\right)^{2}}$, we get $\lambda_{\max }^{\prime} \leq \frac{\lambda_{\min }}{\left(1-\frac{\beta_{2}(\bar{x})}{\sqrt{q(\lfloor\bar{x}\rceil)-q(\bar{x})}} \sqrt{\lambda_{\min }}\right)^{2}}$. Furthermore, we have $(1-\underbrace{\frac{\beta_{2}(\bar{x})}{\sqrt{q(\lfloor\bar{x}\rceil)-q(\bar{x})}} \sqrt{\lambda_{\min }}}_{\geq 0})^{2} \leq 1$, 


$$
\begin{aligned}
& \text { i.e., } \lambda_{\min } \leq \frac{\lambda_{\min }}{\left(1-\frac{\beta_{2}(\bar{x})}{\sqrt{q(\lfloor\bar{x}\rceil)-q(\bar{x})}} \sqrt{\left.\lambda_{\min }\right)^{2}}\right.} \text { and hence } \lambda_{\min }^{\prime}=\lambda_{\min } \text {. This means, } \\
& \frac{1}{\sqrt{\lambda_{\min }^{\prime}}}-\frac{1}{\sqrt{\lambda_{\max }^{\prime}}} \leq \frac{1}{\sqrt{\lambda_{\min }}}-\frac{1-\frac{\beta_{2}(\bar{x})}{\sqrt{q(\lfloor\bar{x}\rceil)-q(\bar{x})}} \sqrt{\lambda_{\min }}}{\sqrt{\lambda_{\min }}}=\frac{\beta_{2}(\bar{x})}{\sqrt{q(\lfloor\bar{x}\rceil)-q(\bar{x})}} .
\end{aligned}
$$

Choose $R:=\frac{1}{\sqrt{\lambda_{\min }^{\prime}}}, r:=\frac{1}{\sqrt{\lambda_{\max }^{\prime}}}$. Then we have $R, r \geq 0, R-r \leq \frac{\beta_{2}(\bar{x})}{\sqrt{q(\lfloor\bar{x}\rceil)-q(\bar{x})}}$ and $R^{2} Q^{\prime}-I_{n} \succeq 0$ since $Q^{\prime}-\lambda_{\min } I_{n} \succeq 0$ and analogously $I_{n}-r^{2} Q^{\prime} \succeq 0$ since $\lambda_{\max } I_{n}-Q^{\prime} \succeq 0$.

- Let $Q^{\prime \prime}$ feasible for (5.5), i.e., $Q^{\prime \prime}=V^{t} \operatorname{Diag}\left(\lambda^{\prime \prime}\right) V$ for some $\lambda^{\prime \prime}>0$. Since $Q^{\prime \prime} \succeq 0$, $Q-Q^{\prime \prime} \succeq 0$, this means, $0 \leq \lambda_{i}^{\prime \prime} \leq \lambda_{i}$ for all $i=1, \ldots, n$. We show $Q^{\prime}-Q^{\prime \prime} \succeq 0$, which implies $(\lfloor\bar{x}\rceil-\bar{x})^{t} Q^{\prime}(\lfloor\bar{x}\rceil-\bar{x}) \geq(\lfloor\bar{x}\rceil-\bar{x})^{t} Q^{\prime \prime}(\lfloor\bar{x}\rceil-\bar{x})$ :

assume that there is a $j \in\{1, \ldots, n\}$ such that $\lambda_{j}^{\prime}<\lambda_{j}^{\prime \prime} \leq \lambda_{j}$, i.e.,

$$
\lambda_{j}^{\prime}=\frac{\lambda_{\min }}{\left(1-\frac{\beta_{2}(\bar{x})}{\sqrt{q(\lfloor\bar{x}\rceil)-q(\bar{x})}} \sqrt{\lambda_{\min }}\right)^{2}}<\lambda_{j}^{\prime \prime} .
$$

Since $\lambda_{i}^{\prime \prime} \leq \lambda_{i}$ for all $i=1, \ldots, n$ we know that $\lambda_{\min }^{\prime \prime}:=\min _{i} \lambda_{i}^{\prime \prime}$ fulfills $\lambda_{\min }^{\prime \prime} \leq \lambda_{\min }$. But this means,

$$
\frac{1}{\sqrt{\lambda_{\min }^{\prime \prime}}}-\frac{1}{\sqrt{\lambda_{\max }^{\prime \prime}}} \geq \frac{1}{\sqrt{\lambda_{\min }}}-\frac{1}{\sqrt{\lambda_{j}^{\prime \prime}}}>\frac{1}{\sqrt{\lambda_{\min }}}-\frac{1-\frac{\beta_{2}(\bar{x})}{\sqrt{q(\lfloor\bar{x}\rceil)-q(\bar{x})}}}{\sqrt{\lambda_{\min }}}=\frac{\beta_{2}(\bar{x})}{\sqrt{q(\lfloor\bar{x}\rceil)-q(\bar{x})}} .
$$

Which means that $E\left(Q^{\prime \prime}, \bar{x}\right)$ is not $\left(\frac{\beta_{2}(\bar{x})}{\sqrt{q(\lfloor\bar{x})-q(\bar{x})}}, 2\right)$-quasi-round and hence there cannot be $r, R \geq 0$ such that $\left(Q^{\prime \prime}, r, R\right)$ is a feasible solution to (5.5).

As $Q^{\prime}$ is feasible for (5.5) it is also feasible for (5.4). Note however that (5.5) has two stricter constraints than (5.4) and therefore there might be better solutions to (5.4) than $Q^{\prime}$ : on the one hand we restrict $Q^{\prime}$ to have the same eigenvectors as $Q$. This means that there might be a solution to (5.4) with different eigenvectors that gives a better bound,compare Example 5.23. But we only see a chance to solve (5.4) efficiently if we fix the eigenvectors beforehand and fixing them to anything else than the eigenvectors of $Q$ would mean that the heuristic would not be able to set $Q^{\prime}=Q$ if $Q$ itself is quasi-round. On the other hand, we restrict $Q^{\prime}$ to be $\left(\frac{\beta_{2}(\bar{x})}{\sqrt{q(\lfloor\bar{x}\rceil)-q(\bar{x})}}, 2\right)$-quasi-round which is stricter than the $\left(\frac{\beta_{2}(\bar{x})}{\sqrt{q^{\prime}(\lfloor\bar{x}\rceil)-q(\bar{x})}}, 2\right)$-quasi-roundness demanded in (5.4) as $q^{\prime}(\lfloor\bar{x}\rceil) \leq q(\lfloor\bar{x}\rceil)$. This means that there might be a matrix $Q^{\prime \prime}$ with the same eigenvectors as $Q$, that is feasible to (5.4) but not to (5.5) and that gives a better bound than $Q^{\prime}$, compare Example 5.24. Even though we might waste some potential here we are using this heuristic since it is easy to compute, which would not be the case if we required $E\left(Q^{\prime}, \bar{x}\right)$ to be $\left(\frac{\beta_{2}(\bar{x})}{\sqrt{q^{\prime}(\lfloor\bar{x}\rceil)-q(\bar{x})}}, 2\right)$-quasi-round since we do not know $q^{\prime}(\lfloor\bar{x}\rceil)$ in advance, and the computational results show clearly that this fast computation more than compensates for the weaker bounds. 
Example 5.23. It is possible that a $\left(\frac{\beta_{2}(\bar{x})}{\sqrt{q^{\prime \prime}(\lfloor\bar{x}\rceil)-q(\bar{x})}}, 2\right)$-quasi-round ellipsoid $Q^{\prime \prime}$ that does not have the same eigenvectors as $Q$ gives a better bound than the one obtained by Lemma 5.22: Consider Figure 5.8: we see the level sets $\sqrt{q^{\prime}(\lfloor\bar{x}\rceil)-q(\bar{x})} E\left(Q^{\prime}, \bar{x}\right)$ (dashed) and $\sqrt{q^{\prime \prime}(\lfloor\bar{x}\rceil)-q(\bar{x})} E\left(Q^{\prime \prime}, \bar{x}\right)$ (solid and light gray), where $Q^{\prime}$ is as given by Lemma 5.22 and $Q^{\prime \prime}$ has slightly different eigenvectors compared to $Q$ and $Q^{\prime}$. Both ellipsoids are $\left(\frac{\beta_{2}(\bar{x})}{\sqrt{q^{\prime}(\lfloor\bar{x}\rceil)-q(\bar{x})}}, 2\right)$ $\operatorname{lor}\left(\frac{\beta_{2}(\bar{x})}{\sqrt{q^{\prime \prime}(\lfloor\bar{x}\rceil)-q(\bar{x})}}, 2\right)$ - respectively) quasi-round w.r.t. $\bar{x}$. We see in the closeup that $Q^{\prime \prime}$ gives a better bound than $Q^{\prime}$.
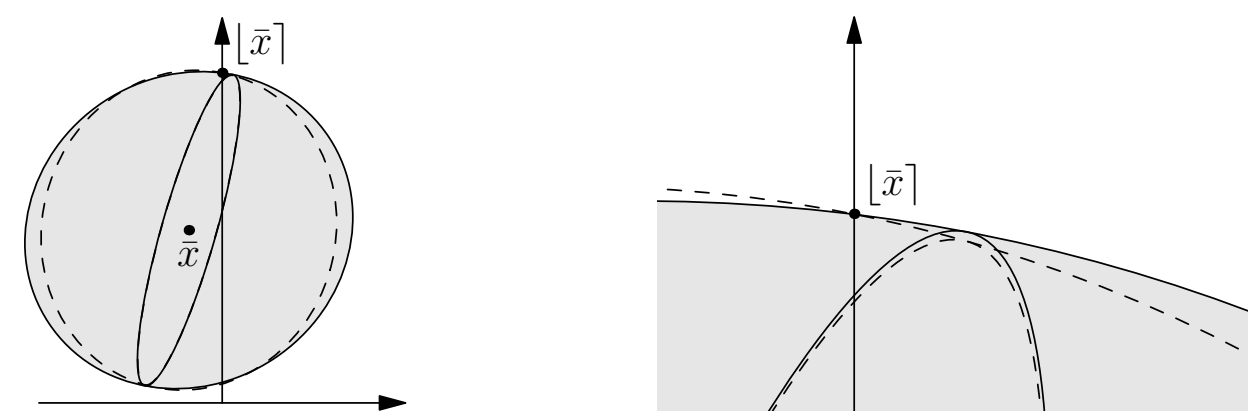

Figure 5.8.: There might be a better approximation (light gray) having the Strong Rounding Property with different eigenvectors as $Q$ than $E\left(Q^{\prime}, \bar{x}\right)$ as determined by Lemma 5.22 (dashed).

Example 5.24. We show in this example that the stricter restriction to be $\left(\frac{\beta_{2}(\bar{x})}{\sqrt{q(\lfloor\bar{x})-q(\bar{x})}}, 2\right)$ quasi-round in (5.5) might lead to underestimators $Q^{\prime}$ with level sets $\sqrt{q^{\prime}(\lfloor\bar{x}\rceil)-q(\bar{x})} E\left(Q^{\prime}, \bar{x}\right)$ that are too round:

Consider Figure 5.9: in the left picture the light gray ellipsoid is $\sqrt{q^{\prime}(\lfloor\bar{x}\rceil)-q(\bar{x})} E\left(Q^{\prime}, \bar{x}\right)$ as determined by Lemma 5.22 and the dark gray ellipsoid is $\sqrt{q^{\prime}(\lfloor\bar{x}\rceil)-q(\bar{x})} E(Q, \bar{x})$. In the right picture the dark gray ellipsoid is $\sqrt{q^{\prime \prime}(\lfloor\bar{x}\rceil)-q(\bar{x})} E(Q, \bar{x})$ and the light gray one is $\sqrt{q^{\prime \prime}(\lfloor\bar{x}\rceil)-q(\bar{x})} E\left(Q^{\prime \prime}, \bar{x}\right)$, where $Q^{\prime \prime}$ has the same eigenvectors as $Q$ and $E\left(Q^{\prime \prime}, \bar{x}\right)$ is $\left(\frac{\beta_{2}(\bar{x})}{\sqrt{q^{\prime \prime}(\lfloor\bar{x}\rceil)-q(\bar{x})}}, 2\right)$-quasi-round.

If we switch to quasi-axisparallel ellipsoids we observe first that $Q^{\prime}$ does not have to be positive definite, but, as in the case of axisparallel ellipsoids, positive semidefinite is enough. Furthermore, we have seen that $E\left(Q^{\prime}, \bar{x}\right)$ has to be $\frac{\beta(t, \bar{x})}{\sqrt{q^{\prime}(\lfloor\bar{x}\rceil)-q(\bar{x})}}$-quasi-axisparallel w.r.t. $\bar{x}$ to guarantee the Strong Rounding Property.

Lemma 5.25. The tightest lower bound we can obtain by underestimating $q$ by $q^{\prime}(x):=$ $q(\bar{x})+(x-\bar{x})^{t} Q^{\prime}(x-\bar{x})$ with $\beta(t, \bar{x})$-quasi-axisparallel level sets $\sqrt{z-q(\bar{x})} E\left(Q^{\prime}, \bar{x}\right)$ for all 

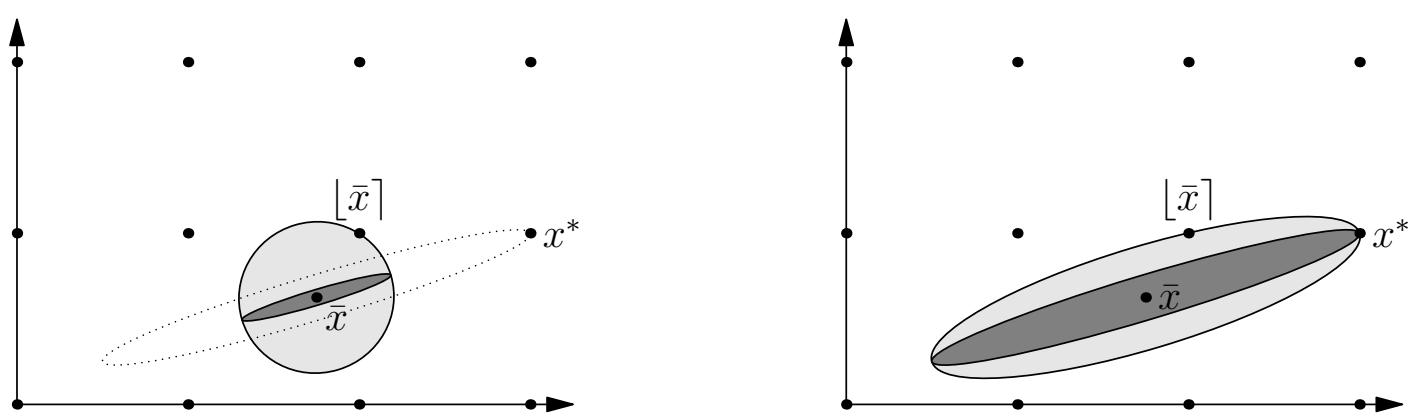

Figure 5.9.: There might be better approximations (right picture) having the Strong Rounding Property and keeping the eigenvectors of $Q$ than $E\left(Q^{\prime}, \bar{x}\right)$ as determined by Lemma 5.22 (left picture).

levels $q(\bar{x}) \leq z \leq q^{\prime}(\lfloor\bar{x}\rceil)$ is given by the optimal objective value of the problem

$$
\begin{array}{cl}
\max & (\lfloor\bar{x}\rceil-\bar{x})^{t} Q^{\prime}(\lfloor\bar{x}\rceil-\bar{x}) \\
\text { s.t. } & Q-Q^{\prime} \succeq 0 \\
& R^{2} Q^{\prime}-\operatorname{Diag}(t) \succeq 0 \\
& \operatorname{Diag}(t)-r^{2} Q^{\prime} \succeq 0 \\
& R-r \leq \frac{\beta(t, \bar{x})}{\sqrt{q^{\prime}(\lfloor\bar{x}\rceil)-q(\bar{x})}} \\
& r, R, t \geq 0 .
\end{array}
$$

An optimal solution $Q_{*}^{\prime}$ defines the underestimator $q^{\prime}(x)=q(\bar{x})+(x-\bar{x})^{t} Q_{*}^{\prime}(x-\bar{x})$ and gives the bound $q(\bar{x})+(\lfloor\bar{x}\rceil-\bar{x})^{t} Q_{*}^{\prime}(\lfloor\bar{x}\rceil-\bar{x})$.

Proof. As mentioned before $\sqrt{z-q(\bar{x})} E\left(Q^{\prime}, \bar{x}\right)$ is $\beta(t, \bar{x})$-quasi-axisparallel w.r.t. $\bar{x}$ for all $q(\bar{x}) \leq z \leq q^{\prime}(\lfloor\bar{x}\rceil)$, if $E\left(Q^{\prime}, \bar{x}\right)$ is $\frac{\beta(t, \bar{x})}{\sqrt{q^{\prime}(\lfloor\bar{x}\rceil)-q(\bar{x})}}$-quasi-axisparallel w.r.t. $\bar{x}$. This is if there exist $t \geq 0, r, R \in \mathbb{R}_{0}^{+}$such that $r \cdot E(\operatorname{Diag}(t), \bar{x}) \subseteq E\left(Q^{\prime}, \bar{x}\right) \subseteq R \cdot E(\operatorname{Diag}(t), \bar{x})$ and $R-r \leq \frac{\beta(t, \bar{x})}{\sqrt{q^{\prime}(\lfloor\bar{x}\rceil)-q(\bar{x})}}$. This is equivalent to $\operatorname{Diag}(t)-r^{2} Q^{\prime} \succeq 0, R^{2} Q^{\prime}-\operatorname{Diag}(t) \succeq 0$ and $R-r \leq \frac{\beta(t, \bar{x})}{\sqrt{q^{\prime}(\lfloor\bar{x}\rceil)-q(\bar{x})}}$.

The only difference to problem (5.4) is that we have the additional variable $t$, which makes the problem even harder since $\beta(t, \bar{x})$ is not a constant as $\beta_{2}(\bar{x})$ but depends nonlinearly on $t$. On the other hand since we know that axisparallel as well as quasi-round ellipsoids are quasiaxisparallel, an optimal solution to problem (5.6) would give a bound that is not worse than the bounds given by (5.3) and (5.4). So we hope that even a heuristic solution to problem (5.6) might lead to good bounds.

As already mentioned the problem is that we now have the additional variable $t$ which keeps us from using the same heuristic as in the quasi-round case. The idea is therefore to fix a reasonable $\hat{t}$ first and then continue as in the quasi-round case. By using the transformation $X \mapsto \operatorname{Diag}(\hat{t})^{-\frac{1}{2}} X \operatorname{Diag}(\hat{t})^{-\frac{1}{2}}$ and the approximation $R-r \leq \frac{\beta(t, \bar{x})}{\sqrt{q(\lfloor\bar{x}])-q(\bar{x})}}$ as before we get the problem 


$$
\begin{array}{cl}
\max & (\lfloor\bar{x}\rceil-\bar{x})^{t} \operatorname{Diag}(\hat{t})^{-\frac{1}{2}} Q^{\prime \prime} \operatorname{Diag}(\hat{t})^{-\frac{1}{2}}(\lfloor\bar{x}\rceil-\bar{x}) \\
\text { s.t. } & \operatorname{Diag}(\hat{t})^{-\frac{1}{2}} Q \operatorname{Diag}(\hat{t})^{-\frac{1}{2}}-Q^{\prime \prime} \succeq 0 \\
& R^{2} Q^{\prime \prime}-I_{n} \succeq 0 \\
& I_{n}-r^{2} Q^{\prime \prime} \succeq 0 \\
& R-r \leq \frac{\beta(\hat{t}, \bar{x})}{\sqrt{q(\lfloor\bar{x}\rceil)-q(\bar{x})}} \\
& r, R, t \geq 0
\end{array}
$$

which has exactly the form of (5.4) and which we solve therefore by Lemma 5.22:

The approximation $Q^{\prime \prime}$ has the same eigenvectors as $\widetilde{Q}:=\operatorname{Diag}(\hat{t})^{-\frac{1}{2}} Q \operatorname{Diag}(\hat{t})^{-\frac{1}{2}}$ and its eigenvalues are given by

$$
\widetilde{\lambda}_{i}^{\prime}=\min \left\{\widetilde{\lambda}_{i}, \frac{\tilde{\lambda}_{\text {min }}}{\left(1-\frac{\beta(\hat{t}, \bar{x})}{\sqrt{q(\lfloor\bar{x}\rceil)-q(\bar{x})}} \sqrt{\widetilde{\lambda}_{\min }}\right)^{2}}\right\} \quad \forall i=1, \ldots, n
$$

with $\widetilde{\lambda}_{i}, \ldots, \widetilde{\lambda}_{n}$ the eigenvalues of $\widetilde{Q}$ and $\widetilde{\lambda}_{\min }:=\min _{i=1, \ldots, n} \widetilde{\lambda}_{i}$.

Then we get a heuristic solution $Q^{\prime}(\hat{t})$ to (5.6) by the reverse transformation $Q^{\prime}(\hat{t})=\operatorname{Diag}(\hat{t})^{\frac{1}{2}} Q^{\prime \prime} \operatorname{Diag}(\hat{t})^{\frac{1}{2}}$.

Note that if $\hat{t}_{i}=0$ for some $i \in\{1, \ldots, n\} \beta(\hat{t}, \bar{x})=0$ and therefore we are done since this means that we use $Q^{\prime}=\operatorname{Diag}(\hat{t})$ to approximate $q$. Hence the transformation $X \mapsto$ $\operatorname{Diag}(\hat{t})^{-\frac{1}{2}} X \operatorname{Diag}(\hat{t})^{-\frac{1}{2}}$ is well-defined since we only use it for $\hat{t}>0$.

If we consider again the case that $Q$ itself is already $\left(\frac{\beta(t, \bar{x})}{\sqrt{q(\lfloor\bar{x}\rceil-q(\bar{x})}}, 2\right)$-quasi-axisparallel, we need not get $Q$ by performing this heuristic: even though we keep the eigenvectors it might happen, that we choose the "wrong" $\hat{t}$, with respect to whom $Q$ is not quasi-axisparallel and hence we have to adapt the eigenvalues.

If we choose an optimal solution to (5.3) for $\hat{t}$, it is obvious that the bound given by (5.7) is always at least as good as the one given by (5.3). If we choose any other $t$ we cannot say in general how the heuristic quasi-axisparallel bound performs in comparison to the other bounds. Also we cannot say how the heuristic quasi-axisparallel bound behaves compared to the heuristic quasi-round bound.

In section 5.5 we compare the bounds we get by the different shapes theoretically, in an example and by some computational experiments. 


\subsection{Lower bounds to use in a branch-and-bound approach}

In this section we adapt the lower bounds obtained in the last section to the special branchand-bound setting.

The branch-and-bound scheme we use is the one introduced by Buchheim et al. [2012a] and works as follows: the variables are fixed in a predefined order $x_{1}, x_{2}, \ldots, x_{n}$. This has the following advantage: if $x_{1}, \ldots, x_{d}$ are fixed to $r_{1}, \ldots, r_{d}$ we are left with the problem

$$
\begin{array}{rll}
(C Q I P)_{r}^{d} & \min & q_{r}^{d}(x):=x^{t} Q^{d} x+\left(L^{d}(r)\right)^{t} x+c^{d}(r) \\
& \text { s.t. } \quad x \in \mathbb{Z}^{n-d}
\end{array}
$$

where

$$
\begin{aligned}
c^{d}(r) & :=\sum_{i=1}^{d} L_{i} r_{i}+\sum_{i=1}^{d} \sum_{j=1}^{d} q_{i j} r_{i} r_{j} \\
L_{j-d}^{d}(r) & :=L_{j}+2 \sum_{i=1}^{d} q_{i j} r_{i} \quad \forall j=d+1, \ldots, n
\end{aligned}
$$

and $Q^{d}$ is obtained from $Q$ by deleting the first $d$ rows and columns. This means in particular that $Q^{d}$ does not depend on the values $r_{1}, \ldots, r_{d}$. Using $q_{r}^{d}$ we have $q\left(r_{1}, \ldots, r_{d}, x\right)=q_{r}^{d}(x)$ for all $x \in \mathbb{R}^{n-d}$.

This means that in each depth $d$ of the branch-and-bound tree we have to solve a (lower dimensional) problem of the original type $(C Q I P)$ : note that $Q^{d}$ is still positive definite.

Since $Q^{d}$ is the same for all nodes in depth $d$ of the branch-and-bound tree it is preferable for computational reasons to use only one fixed matrix $Q_{d}^{\prime}$, for each depth $d$ to derive a lower bound (compare Buchheim et al. [2012a]). But the center $\bar{x}$ is different in the different nodes of depth $d$. This means first of all, that we have to make sure that $E\left(Q^{\prime}, \bar{x}\right)$ is axisparallel, quasi-round or quasi-axisparallel w.r.t. each $\bar{x}$ in this depth, where we can assume, due to the usually large number of nodes in each depth, that each $\bar{x} \in \mathbb{R}^{n}$ is possible. This is no additional constraint in the axisparallel case, but we have seen that $\min _{x \in \mathbb{R}^{n}} \beta_{2}(\bar{x})=0$ and $\min _{x \in \mathbb{R}^{n}} \beta(t, \bar{x})=0$ for all $t \geq 0$. This means that the only ellipsoid that is $\left(\beta_{2}(\bar{x}), 2\right)$-quasi-round w.r.t. $\bar{x}$ for each $\bar{x} \in \mathbb{R}^{n}$ is the sphere and the only ellipsoids that are $\beta(t, \bar{x})$-quasi-axisparallel w.r.t. $\bar{x}$ for all $\bar{x} \in \mathbb{R}^{n}$ are axisparallel ellipsoids. Hence it only makes sense to consider axisparallel ellipsoids $E\left(Q^{\prime}, \bar{x}\right)$ in this section.

Even though axisparallelity does not depend on the center point $\bar{x}, t^{*}$ that gives the optimal solution in problem (5.3) depends on the center $\bar{x}$ and not only on the matrix $Q$, compare also the examples in Section 5.5.

This means, we need to find a $t \geq 0$ that gives us a good lower bound for all $\bar{x}$ in a certain depth $d$ in the branch-and-bound tree, depending only on $Q^{d}$. By good we mean that we want to close as much as possible of the relative gap left by the trivial lower bound $q(\bar{x})$. Recall that the bound we get by using $Q^{\prime}$ is $q^{\prime}(\lfloor\bar{x}\rceil)$, since we allow only underestimators having the Strong Rounding Property. This means, we want to maximize

$$
\frac{q^{\prime}(\lfloor\bar{x}\rceil)-q(\bar{x})}{q\left(x^{*}\right)-q(\bar{x})}
$$


Since we do not know $x^{*}$ we use $q(\lfloor\bar{x}\rceil)$ as an upper bound and hence maximize

$$
\Delta\left(Q^{\prime}, \bar{x}\right):=\frac{q^{\prime}(\lfloor\bar{x}\rceil)-q(\bar{x})}{q(\lfloor\bar{x}\rceil)-q(\bar{x})}
$$

(where we assume as always that $\bar{x} \notin \mathbb{Z}^{n}$ ).

We show the following properties of $\Delta\left(Q^{\prime}, \bar{x}\right)$ :

Lemma 5.26. Let $Q-Q_{i}^{\prime} \succeq 0, q_{i}^{\prime}:=q(\bar{x})+(q-\bar{x})^{t} Q_{i}^{\prime}(x-\bar{x})$ have the Strong Rounding Property for $i=1,2$ and let $\bar{x} \in \mathbb{R}^{n} \backslash \mathbb{Z}^{n}$. Then the following holds:

(i) $\Delta\left(Q_{1}^{\prime}, \bar{x}\right)=\frac{\|\lfloor\bar{x}\rceil-\bar{x}\|_{Q_{1}^{\prime}}^{2}}{\|\lfloor\bar{x}\rceil-\bar{x}\|_{Q}^{2}}$

(ii) If $Q_{1}^{\prime}-Q_{2}^{\prime} \succeq 0$, we get $\Delta\left(Q_{1}^{\prime}, \bar{x}\right) \geq \Delta\left(Q_{2}^{\prime}, \bar{x}\right)$. If $Q_{1}^{\prime}-Q_{2}^{\prime} \succ 0$, we get $\Delta\left(Q_{1}^{\prime}, \bar{x}\right)>\Delta\left(Q_{2}^{\prime}, \bar{x}\right)$.

(iii) $\Delta\left(Q_{1}^{\prime}, \bar{x}\right)=\Delta\left(Q_{1}^{\prime}, \bar{x}+z\right)$ for all $z \in \mathbb{Z}^{n}$.

Proof. $\quad$ (i) $\Delta\left(Q_{1}^{\prime}, \bar{x}\right)=\frac{q^{\prime}(\lfloor\bar{x}\rceil)-q(\bar{x})}{q(\lfloor\bar{x}\rceil)-q(\bar{x})}=\frac{\left(\lfloor\bar{x}\rceil-\bar{x} t^{t} Q_{1}^{\prime}(\lfloor\bar{x}\rceil-\bar{x})\right.}{(\lfloor\bar{x}\rceil-\bar{x})^{t} Q(\lfloor\bar{x}\rceil-\bar{x})}=\frac{\|\lfloor\bar{x}\rceil-\bar{x}\|_{Q_{1}^{\prime}}^{2}}{\|\lfloor\bar{x}\rceil-\bar{x}\|_{Q}^{2}}$

(ii) $Q_{1}^{\prime}-Q_{2}^{\prime} \succeq 0$ means $(\lfloor\bar{x}\rceil-\bar{x})^{t} Q_{1}^{\prime}(\lfloor\bar{x}\rceil-\bar{x}) \geq(\lfloor\bar{x}\rceil-\bar{x})^{t} Q_{2}^{\prime}(\lfloor\bar{x}\rceil-\bar{x})$ and $Q_{1}^{\prime}-Q_{2}^{\prime} \succ 0$ means $(\lfloor\bar{x}\rceil-\bar{x})^{t} Q_{1}^{\prime}(\lfloor\bar{x}\rceil-\bar{x})>(\lfloor\bar{x}\rceil-\bar{x})^{t} Q_{2}^{\prime}(\lfloor\bar{x}\rceil-\bar{x})$.

(iii) Since $\lfloor\bar{x}+z\rceil=\lfloor\bar{x}\rceil+z$ for all $z \in \mathbb{Z}^{n}$, we have $\Delta\left(Q_{1}^{\prime}, \bar{x}+z\right) \stackrel{(i)}{=} \frac{\|\lfloor\bar{x}\rceil+z-(\bar{x}+z)\|_{Q_{1}^{\prime}}^{2}}{\|\lfloor\bar{x}\rceil+z-(\bar{x}+z)\|_{Q}^{2}}=$ $\frac{\|\lfloor\bar{x}\rceil-\bar{x}\|_{Q_{1}^{\prime}}^{2}}{\|\lfloor\bar{x}\rceil-\bar{x}\|_{Q}^{2}}=\Delta\left(Q_{1}^{\prime}, \bar{x}\right)$.

As we want to maximize $\Delta\left(Q^{\prime}, \bar{x}\right)$ for all $\bar{x} \in \mathbb{R}^{n} \backslash \mathbb{Z}^{n}$ we have basically two possibilities: we could either maximize the worst-case $\Delta$ or the average-case $\Delta$. We start with the worst-case analysis.

\subsubsection{Worst-case analysis}

Finding a $Q^{\prime}$ that is best in the worst case means solving the following problem

$$
\begin{array}{ll}
\max & \inf _{\bar{x} \in \mathbb{R}^{n} \backslash \mathbb{Z}^{n}} \Delta(\operatorname{Diag}(t), \bar{x}) \\
\text { s.t. } & Q-\operatorname{Diag}(t) \succeq 0 \\
& t \geq 0:
\end{array}
$$

recall that we only allow axisparallel $Q^{\prime}$.

Lemma 5.27. Problem (5.8) is equivalent to

$$
\begin{array}{cl}
\max & \gamma \\
\text { s.t. } & Q-\operatorname{Diag}(t) \succeq 0 \\
& \operatorname{Diag}(t)-\gamma Q \succeq 0 .
\end{array}
$$


Proof. As $Q \succ 0$, there exists $Q^{1 / 2}$ positive definite, s.t. $Q=Q^{1 / 2} Q^{1 / 2}$.

Then $Q^{-1 / 2} \operatorname{Diag}(t) Q^{-1 / 2}$ is positive semidefinite for all $t \geq 0$. Denote by $\widetilde{U} t \widetilde{D}$ the eigendecompositon of $Q^{-1 / 2} \operatorname{Diag}(t) Q^{-1 / 2}$ and by $\lambda_{\min }\left(Q^{-1 / 2} \operatorname{Diag}(t) Q^{-1 / 2}\right)=\min _{i} \widetilde{d}_{i} \geq 0$ the smallest eigenvalue of $Q^{-1 / 2} \operatorname{Diag}(t) Q^{-1 / 2}$. We have

$$
\begin{aligned}
& \inf _{\bar{x} \in \mathbb{R}^{n} \backslash \mathbb{Z}^{n}} \Delta(\operatorname{Diag}(t), \bar{x}) \quad \stackrel{5.26(i)}{=} \quad \inf _{\bar{x} \in \mathbb{R}^{n} \backslash \mathbb{Z}^{n}} \frac{(\lfloor\bar{x}\rceil-\bar{x})^{t} \operatorname{Diag}(t)(\lfloor\bar{x}\rceil-\bar{x})}{(\lfloor\bar{x}\rceil-\bar{x})^{t} Q(\lfloor\bar{x}\rceil-\bar{x})} \\
& y=\underline{\lfloor\bar{x}\rceil-\bar{x}} \inf _{y \in \mathbb{R}^{n} \backslash\{0\}} \frac{y^{t} \operatorname{Diag}(t) y}{y^{t} Q y} \\
& z=\underline{Q}^{1 / 2} y \quad \inf _{z \in \mathbb{R}^{n} \backslash\{0\}} \frac{z^{t} Q^{-1 / 2} \operatorname{Diag}(t) Q^{-1 / 2} z}{z^{t} z} \\
& =\quad \inf _{z \in \mathbb{R}^{n} \backslash\{0\}} \frac{z^{t} \widetilde{U^{t}} \widetilde{D} \widetilde{U} z}{z^{t} z} \\
& w=\widetilde{U} z \quad \inf _{w \in \mathbb{R}^{n} \backslash\{0\}} \frac{w \top \widetilde{D} w}{w^{t} \widetilde{U} \widetilde{U}^{t} w} \\
& =\quad \inf _{w \in \mathbb{R}^{n} \backslash\{0\}} \frac{\sum_{i=1}^{n} w_{i}^{2} \widetilde{d}_{i}}{\sum_{i=1}^{n} w_{i}^{2}} \\
& =\quad \lambda_{\min }\left(Q^{-1 / 2} \operatorname{Diag}(t) Q^{-1 / 2}\right) .
\end{aligned}
$$

As $\lambda_{\min }\left(Q^{-1 / 2} \operatorname{Diag}(t) Q^{-1 / 2}\right) \geq \gamma \Leftrightarrow Q^{-1 / 2} \operatorname{Diag}(t) Q^{-1 / 2}-\gamma I_{n} \succeq 0 \Leftrightarrow \operatorname{Diag}(t)-\gamma Q \succeq 0$ this means that Problem (5.8) is equivalent to Problem (5.9). (The constraint $t \geq 0$ can be omitted as $(\gamma, t)=(0,0)$ is a feasible solution, an optimal solution $\left(\gamma^{*}, t^{*}\right)$ hence satisfies $\gamma^{*} \geq 0$ and hence $\operatorname{Diag}\left(t^{*}\right) \succeq \gamma^{*} Q \succeq 0$ implies $t^{*} \geq 0$.)

Lemma 5.27 shows that the worst-case best axisparallel ellipsoid can be found efficiently by solving a SDP.

\subsubsection{Average-case analysis}

Since we have typically a lot of different nodes in depth $d$ of the branch-and-bound tree, an average-case best axisparallel ellipsoid might be a better choice than a worst-case best one. As we have seen in Lemma 5.26 (iii) $\Delta\left(Q^{\prime}, \bar{x}\right)$ is periodic in $\bar{x}$. Therefore we do not consider $\bar{x} \in \mathbb{R}^{n} \backslash \mathbb{Z}^{n}$ but $\bar{x} \in[0,1]^{n} \backslash\{0,1\}^{n}$. For large branch-and-bound trees it is reasonable to assume that $\bar{x}$ is uniformly distributed in $[0,1]^{n}$. This means that we find an average-case best underestimator $q^{\prime}$ to $q$ with axisparallel level sets by solving

$$
\begin{array}{ll}
\max & \int_{[0,1]^{n} \backslash\{0,1\}^{n}} \Delta(\operatorname{Diag}(t), \bar{x}) d \bar{x} \\
\text { s.t. } & Q-\operatorname{Diag}(t) \succeq 0 \\
& t \geq 0 .
\end{array}
$$

Lemma 5.28. Problem (5.10) is equivalent to

$$
\begin{array}{cl}
\max & \beta^{t} t \\
\text { s.t. } & Q-\operatorname{Diag}(t) \succeq 0 \\
& t \geq 0
\end{array}
$$


for $\beta_{i}:=\int\left[-\frac{1}{2}, \frac{1}{2}\right]^{n} \backslash\{0\} \frac{y_{i}^{2}}{y^{t} Q y} d y$.

Proof. We have seen in Lemma 5.26 (i) that $\Delta(\operatorname{Diag}(t), \bar{x})=\frac{\|\lfloor\bar{x}\rceil-\bar{x}\|_{t}^{2}}{\|\lfloor\bar{x}]-\bar{x}\|_{Q}^{2}}$. Substituting $y:=$ $\lfloor\bar{x}\rceil-\bar{x}$ we get $y \in\left[-\frac{1}{2}, \frac{1}{2}\right]^{n} \backslash\{0\}$ and

$$
\Delta(\operatorname{Diag}(t), \bar{x})=\frac{\|y\|_{t}^{2}}{\|y\|_{Q}^{2}}=\frac{\sum_{i=1}^{n} t_{i} y_{i}^{2}}{y^{t} Q y}=\sum_{i=1}^{n} t_{i} \frac{y_{i}^{2}}{y^{t} Q y} .
$$

This means,

$$
\int_{[0,1]^{n} \backslash\{0,1\}^{n}} \Delta(\operatorname{Diag}(t), \bar{x}) d \bar{x}=\int_{\left[-\frac{1}{2}, \frac{1}{2}\right]^{n} \backslash\{0\}} \sum_{i=1}^{n} t_{i} \frac{y_{i}^{2}}{y^{t} Q y} d y=\sum_{i=1}^{n} t_{i} \underbrace{\int_{\left[-\frac{1}{2}, \frac{1}{2}\right]^{n} \backslash\{0\}} \frac{y_{i}^{2}}{y^{t} Q y} d y}_{=: \beta_{i}} .
$$

Since the coefficients $\beta_{i}$ are independent of $t$ we can calculate them in a preprocessing phase. The resulting problem (5.11) is a SDP and can hence be solved efficiently.

Remark 5.29. Rather than taking the relative gap one could also think of taking the absolute gap $q^{\prime}(\lfloor\bar{x}\rceil)-q(\bar{x})$ as a measure of how good $Q^{\prime}$ is. This leads to the following considerations:

Worst-case:

$$
\begin{array}{ll}
\max & \inf _{x \in \mathbb{R}^{n} \backslash \mathbb{Z}^{n}} \sum_{i=1}^{n}\left(\left\lfloor\bar{x}_{i}\right\rceil-\bar{x}_{i}\right)^{2} t_{i} \\
\text { s.t. } & Q-\operatorname{Diag}(t) \succeq 0 \\
& t \geq 0
\end{array}
$$

Since $Q-\operatorname{Diag}(t) \succeq 0$ implies $q_{i i}-t_{i} \geq 0$ for all $i=1, \ldots, n$, the $t_{i}$ are bound and since furthermore $t_{i} \geq 0$ this means,

$$
\inf _{x \in \mathbb{R}^{n} \backslash \mathbb{Z}^{n}} \sum_{i=1}^{n}\left(\left\lfloor\bar{x}_{i}\right\rceil-\bar{x}_{i}\right)^{2} t_{i}=0 \quad \forall t \geq 0 \quad \text { as } \inf _{x \in \mathbb{R}^{n} \backslash \mathbb{Z}^{n}} \sum_{i=1}^{n}\left(\left\lfloor\bar{x}_{i}\right\rceil-\bar{x}_{i}\right)^{2}=0 .
$$

Hence all axisparallel ellipsoid give the same lower bound in the worst-case and hence this criterion is not helpful in comparing different choices of $Q^{\prime}$.

Average-case:

$$
\begin{array}{lllll}
\max & \int_{\left[-\frac{1}{2}, \frac{1}{2}\right]^{n} \backslash\{0\}} \sum_{i=1}^{n} y_{i}^{2} t_{i} d y \Leftrightarrow \max & \frac{1}{12} \sum_{i=1}^{n} t_{i} \\
\text { s.t. } & Q-\operatorname{Diag}(t) \succeq 0 & & \text { s.t. } & Q-\operatorname{Diag}(t) \succeq 0 \\
& t \geq 0 & & t \geq 0
\end{array}
$$

This means, we get almost the same SDP as in (5.11) except for that here all $\beta_{i}$ are equal. 


\subsection{Comparison of the bounds}

In the last sections we proposed different ways of obtaining underestimators $q^{\prime}$ that have the Strong Rounding Property.

The aim of this section is to compare them on the one hand regarding the resulting lower bound, but on the other hand also regarding their computational effectivity, i.e., regarding the time we need to solve $(C Q I P)$ by a branch-and-bound algorithm using these bounds. This means, we also take the time we need to calculate the bound into consideration.

We start by comparing the resulting lower bounds, where we consider the following ways to compute them:

- best axisparallel: $l b_{a p}$ as defined by Lemma 5.20

- worst-case best axisparallel: $l b_{w c}$ as defined by Lemma 5.27

- average-case best axisparallel: $l b_{a c}$ as defined by Lemma 5.28

- best quasi-round: $l b_{q r}$ as defined by Lemma 5.21 (we only compare this bound theoretically since we are not able to compute it)

- heuristic quasi-round: $l b_{h q r}$ as defined by Lemma 5.22

- best quasi-axisparallel: $l b_{q a p}$ as defined by Lemma 5.25 (we only compare this bound theoretically since we are not able to compute it)

- heuristic quasi-axisparallel: $l b_{h q a p}(\hat{t})$ as defined by problem (5.7) with Lemma 5.22 where we use the following $\hat{t}$ 's as input to (5.7):

$-\hat{t}=t^{a p}$ where $t^{a p}$ is an optimal solution to (5.3), i.e., we use the best axisparallel underestimator: $l b_{\text {hqap }}\left(t^{a p}\right)$

$-\hat{t}=t^{w c}$ where $t^{w c}$ is an optimal solution to (5.9), i.e., we use the worst-case best axisparallel underestimator: $l b_{\text {hqap }}\left(t^{w c}\right)$

- $\hat{t}=t^{a c}$ where $t^{a c}$ is an optimal solution to (5.11), i.e., we use the average-case best axisparallel underestimator: $l b_{\text {hqap }}\left(t^{a c}\right)$

We already mentioned that the best quasi-axisparallel bound is always at least as good as any axisparallel or quasi-round bound. Of course we know that the best quasi-axisparallel or quasi-round bound is never inferior to the corresponding heuristic bound. Furthermore, the best axisparallel bound is at least as good as the worst-case best and the average-case best bound which are also axisparallel. Depending on which $\hat{t}$ we choose for the quasi-axisparallel heuristic we get that using this heuristic is never worse than using Diag $(\hat{t})$ to derive a bound. All together we get the dominances shown in Figure 5.10 where $A \rightarrow B$ means that $A$ gives a bound that is at least as good as the one given by $B$. (The different heights in Figure 5.10 do not have any meaning, but are only for readability.)

We point out here that the fact that the worst-case and the average-case bound compare so badly in Figure 5.10 is of course due to the fact that we use less information to compute them: whereas the other bounds depend on $\bar{x}$ these two only depend on $Q$. Therefore it might be unfair to put them in this comparison. On the other hand we are not able to actually determine 


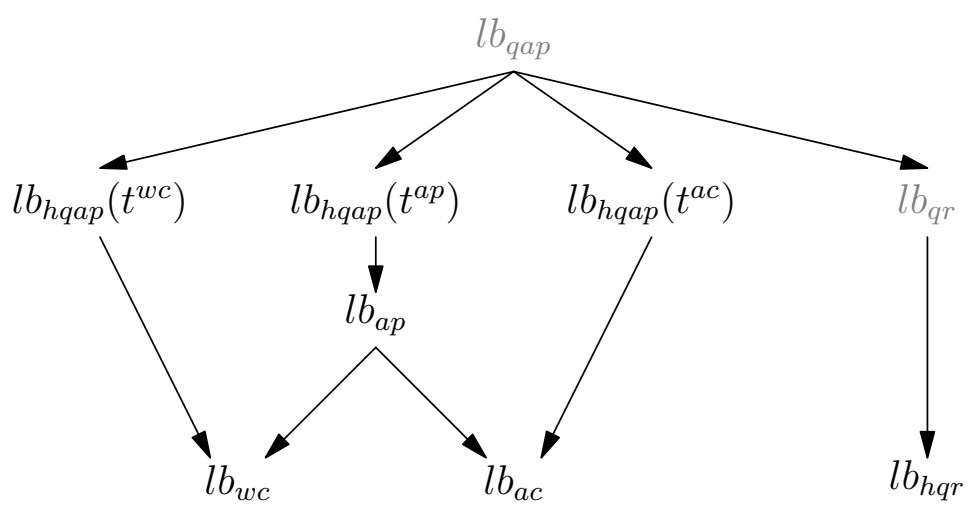

Figure 5.10.: Theoretical dominances between the different bounds.

the best quasi-axisparallel and the best quasi-round ellipsoid, i.e., also these bounds are only in the comparison for the sake of completeness.

To investigate the remaining open questions, i.e., where no arc is shown in Figure 5.10, and to visualize the bounds, we consider the following example:

Example 5.30. Let $Q=\frac{1}{42}\left(\begin{array}{cc}1719 & -476 \\ -476 & 189\end{array}\right)$ and consider

$q_{1}(x):=\left(x-\left(\begin{array}{l}0.2 \\ 0.7\end{array}\right)\right)^{t} Q\left(x-\left(\begin{array}{c}0.2 \\ 0.7\end{array}\right)\right), q_{2}(x):=\left(x-\left(\begin{array}{l}0.9 \\ 0.3\end{array}\right)\right)^{t} Q\left(x-\left(\begin{array}{c}0.9 \\ 0.3\end{array}\right)\right)$

and $q_{3}(x):=\left(x-\left(\begin{array}{c}0.9 \\ 0.4\end{array}\right)\right)^{t} Q\left(x-\left(\begin{array}{c}0.9 \\ 0.4\end{array}\right)\right)$.

The continuous minimizers $\bar{x}_{i}$ can hence easily be seen and we have $q_{i}\left(\bar{x}_{i}\right)=0$ for all $i \in$ $\{1,2,3\}$ :

\begin{tabular}{l|l|l}
\hline$q_{1}(x)$ & $q_{2}(x)$ & $q_{3}(x)$ \\
\hline \hline $\bar{x}_{1}=\left(\begin{array}{l}0.2 \\
0.7\end{array}\right)$ & $\bar{x}_{2}=\left(\begin{array}{l}0.9 \\
0.3\end{array}\right)$ & $\bar{x}_{3}=\left(\begin{array}{c}0.9 \\
0.4\end{array}\right)$ \\
$q_{1}\left(\bar{x}_{1}\right)=0$ & $q_{2}\left(\bar{x}_{2}\right)=0$ & $q_{3}\left(\bar{x}_{3}\right)=0$ \\
\hline
\end{tabular}

Next we determine the integer minimizers $x_{i}^{*}$ by growing the level sets until they reach the first integer point, calculate $q_{i}\left(x_{i}^{*}\right)$ to compare with the bounds in the following and depict the level sets $\mathcal{L}_{\leq, q_{i}}\left(q_{i}\left(x_{i}^{*}\right)\right)$ : 


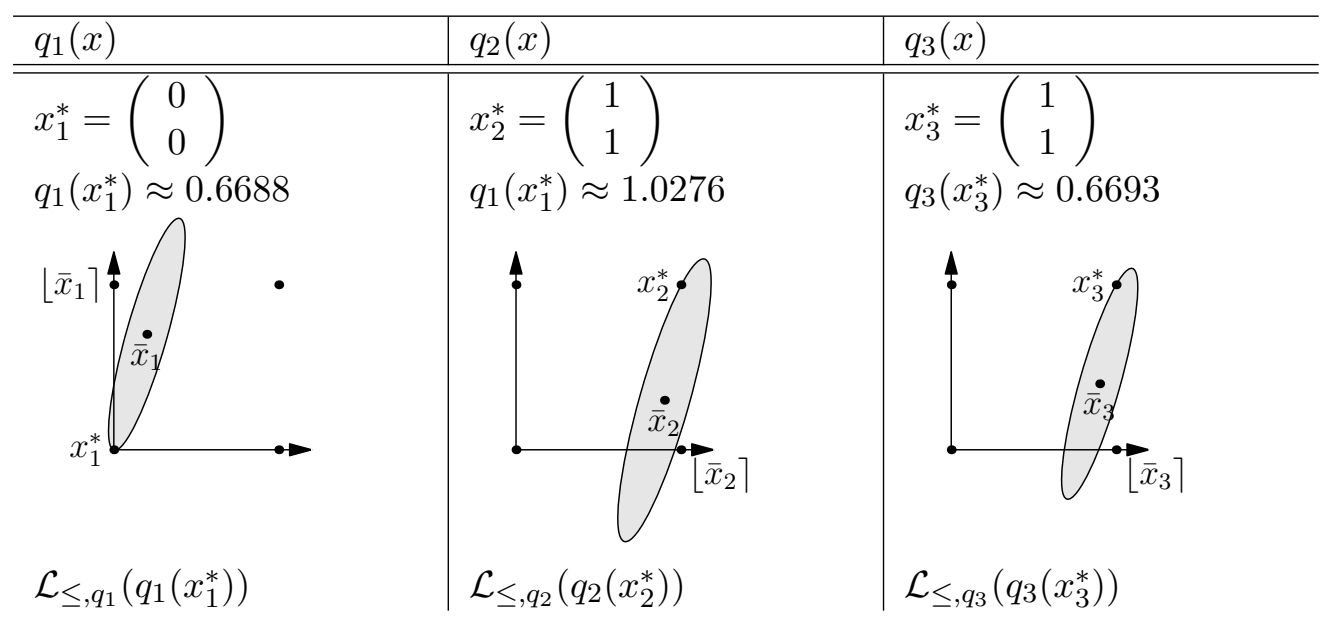

We see that none of the functions has the SRP since $\left\lfloor\bar{x}_{1}\right\rceil=\left(\begin{array}{l}0 \\ 1\end{array}\right) \notin X_{1}^{*},\left\lfloor\bar{x}_{2}\right\rceil=\left(\begin{array}{l}1 \\ 0\end{array}\right) \notin X_{2}^{*}$ and $\left\lfloor\bar{x}_{3}\right\rceil=\left(\begin{array}{l}1 \\ 0\end{array}\right) \notin X_{3}^{*}$.

Now we compare the different bounds mentioned before. In the pictures we show $\mathcal{L}_{\leq, q_{i}}\left(l b_{q_{i}^{\prime}}\right)$ (solid) and in light gray $\mathcal{L}_{\leq, q_{i}^{\prime}}\left(l b_{q_{i}^{\prime}}\right)$ for the different underestimators $q_{i}^{\prime}$. Since $q_{i}^{\prime}$ has the Strong Rounding Property we always observe $\left\lfloor\bar{x}_{i}\right\rceil \in \mathcal{L}_{\leq, q_{i}^{\prime}}\left(l b_{q_{i}^{\prime}}\right)$. The dotted lines depict the level set $\mathcal{L}_{\leq, q_{i}}\left(q_{i}\left(x_{i}^{*}\right)\right)$ corresponding to the integer minimizer for comparison.

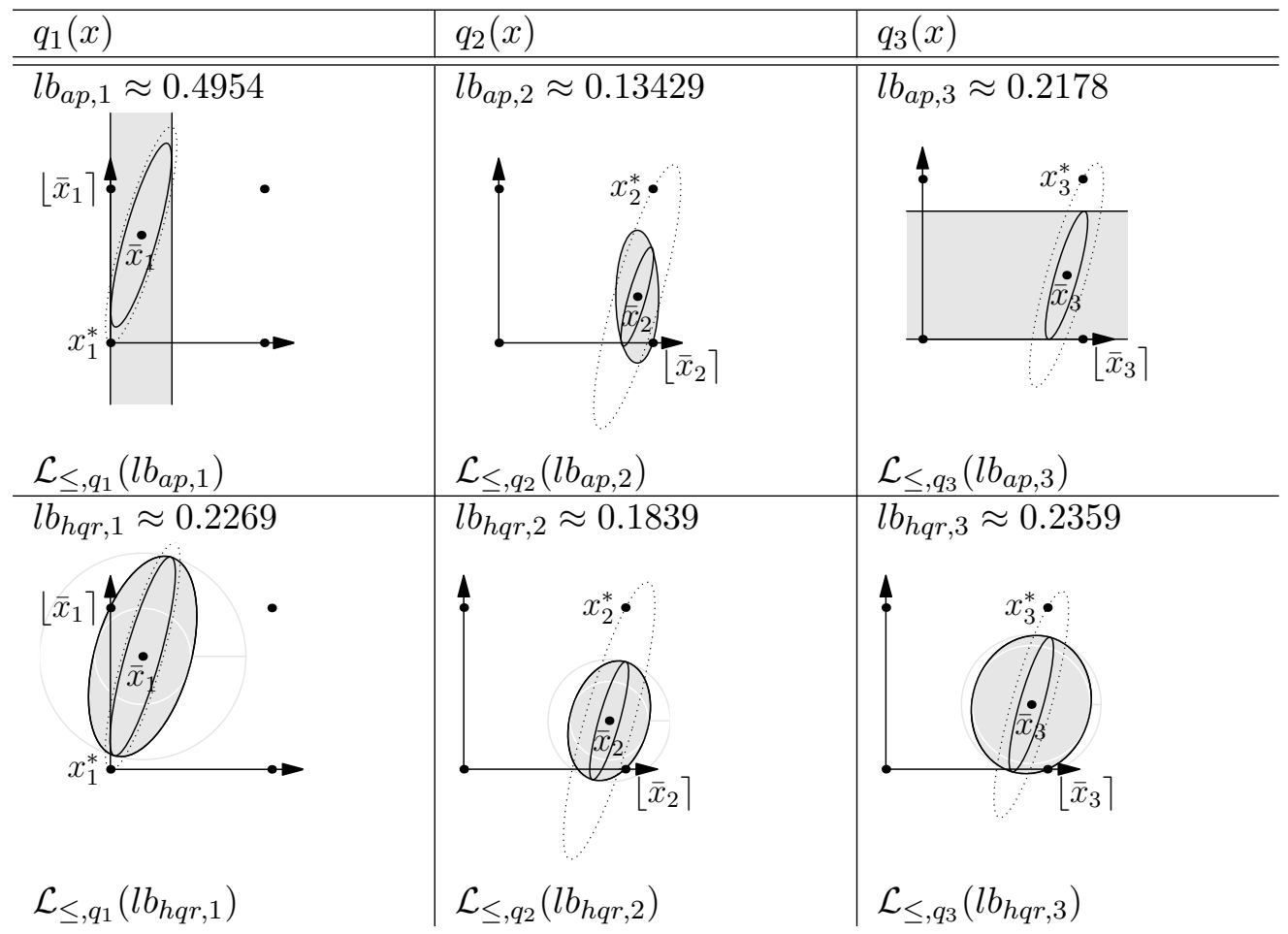




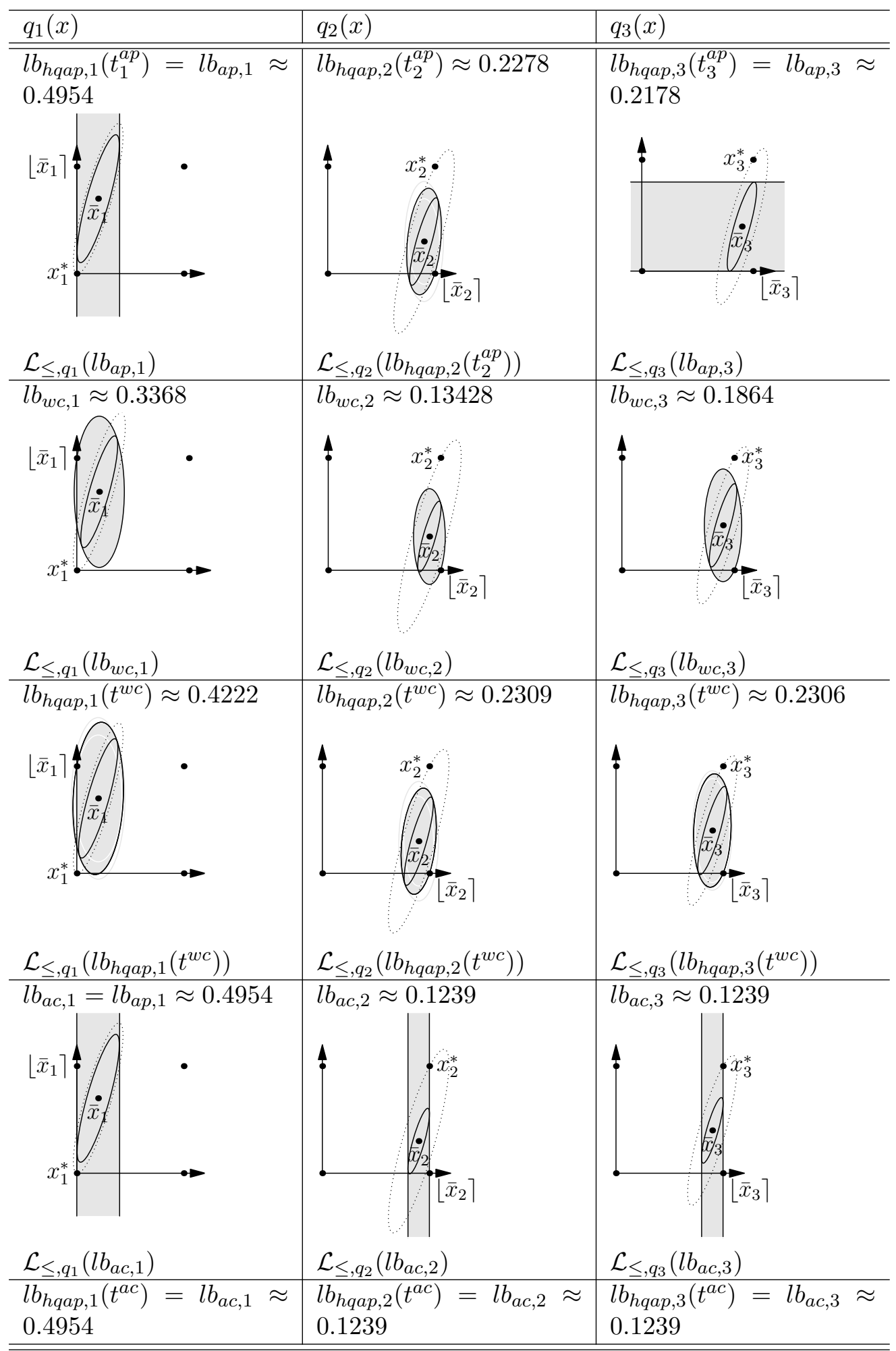


All together we get the following rankings:

\begin{tabular}{|c|c|c|c|c|c|}
\hline \multicolumn{2}{|l|}{$q_{1}(x)$} & \multicolumn{2}{|l|}{$q_{2}(x)$} & \multicolumn{2}{|l|}{$q_{3}(x)$} \\
\hline$q_{1}\left(x_{1}^{*}\right) \approx$ & 0.6688 & $q_{2}\left(x_{2}^{*}\right) \approx$ & 1.0276 & $q_{3}\left(x_{3}^{*}\right) \approx$ & 0.6693 \\
\hline$l b_{a p, 1}=l b_{h q a p, 1}\left(t_{1}^{a p}\right)=$ & & $l b_{h q a p, 2}\left(t^{w c}\right) \approx$ & 0.2309 & $l b_{h q r, 3} \approx$ & 0.2359 \\
\hline$l b_{a c, 1}=l b_{h q a p, 1}\left(t^{a c}\right) \approx$ & 0.4954 & $l b_{h q a p, 2}\left(t_{2}^{a p}\right) \approx$ & 0.2278 & $l b_{h q a p, 3}\left(t^{w c}\right) \approx$ & 0.2306 \\
\hline$l b_{\text {hqap }, 1}\left(t^{w c}\right) \approx$ & 0.4222 & $l b_{h q r, 2} \approx$ & 0.1839 & $l b_{a p, 3}=$ & \\
\hline$l b_{w c, 1} \approx$ & 0.3368 & $l b_{a p, 2} \approx$ & 0.13429 & $l b_{h q a p, 3}\left(t_{3}^{a p}\right) \approx$ & 0.2178 \\
\hline$l b_{h q r, 1} \approx$ & 0.2269 & $l b_{w c, 2} \approx$ & 0.13428 & $l b_{w c, 3} \approx$ & 0.1862 \\
\hline$q_{1}\left(\bar{x}_{1}\right)=$ & 0 & $l b_{a c, 2}=l b_{h q a p, 2}\left(t^{a c}\right) \approx$ & 0.1239 & $l b_{a c, 3}=$ & \\
\hline & & $q_{2}\left(\bar{x}_{2}\right)=$ & 0 . & $\begin{array}{r}l b_{h q a p, 3}\left(t^{a c}\right) \approx \\
q_{3}\left(\bar{x}_{3}\right)=\end{array}$ & $\begin{array}{l}0.1239 \\
0\end{array}$ \\
\hline
\end{tabular}

If we compare these rankings with Figure 5.10, we see that for almost all pairs of bounds where no hierarchy is depicted, depending on $\bar{x}$, either one of them can be better than the other and hence there cannot be any hierarchy between them. The only remaining question is:

Are there examples where $l b_{h q a p}\left(t^{a c}\right)>l b_{h q a p}\left(t^{a p}\right) \geq l b_{a p}$ ?

This situation could not happen in our example, since for $Q$ as given in this example we get

$$
t^{a c}=\left(\begin{array}{c}
\frac{14045}{1134} \\
0
\end{array}\right)
$$

and hence $Q_{h q a p}^{\prime}\left(t^{a c}\right)=Q_{a c}^{\prime}$ and $l b_{h q a p}\left(t^{a c}\right)=l b_{a c} \leq l b_{a p}$.

To answer this remaining question, consider the following example:

Example 5.31.

$$
q(x):=\frac{1}{42}\left(x-\left(\begin{array}{l}
0.1 \\
0.8
\end{array}\right)\right)^{t}\left(\begin{array}{cc}
1033 & -280 \\
-280 & 133
\end{array}\right)\left(x-\left(\begin{array}{l}
0.1 \\
0.8
\end{array}\right)\right) .
$$

For this function we calculate $l b_{\text {hqap }}\left(t^{a c}\right) \approx 0.1070$ and $l b_{a p}=l b_{\text {hqap }}\left(t^{a p}\right)=0.1056$, compare also Figure 5.11.
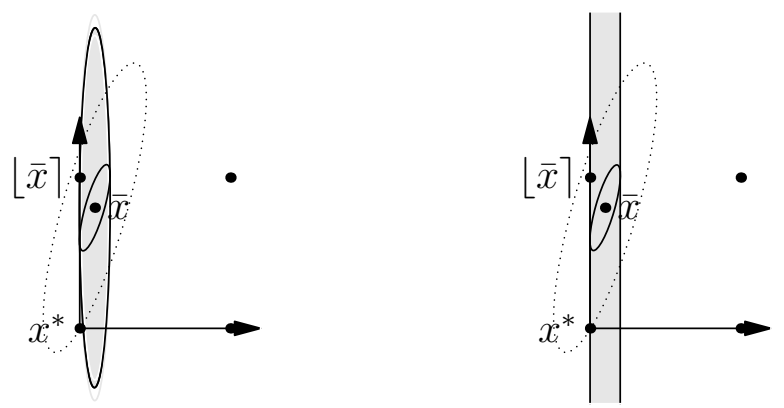

Figure 5.11.: left: $\mathcal{L}_{\leq, q}\left(l b_{h q a p}\left(t^{a c}\right)\right)$ and in light gray $\mathcal{L}_{\leq, q^{\prime}}\left(l b_{h q a p}\left(t^{a c}\right)\right)$, right: $\mathcal{L}_{\leq, q}\left(l b_{a p}\right)$ and in light gray $\mathcal{L}_{\leq, q^{\prime}}\left(l b_{a p}\right)$.

This means, the theoretical hierarchy we showed in Figure 5.10 is complete: for all other pairs of bounds we can find examples for both directions. 
Nevertheless, it might be that some of the bounds perform better than others on average and, as we mentioned before, the time to compute the bounds has to be taken into account too. The most important comparison however is the overall performance in a branch-and-bound tree. To investigate all these questions we do some computational experiments. These experiments are also submitted in Buchheim et al. [2013] and are the work of Christoph Buchheim.

To this end we embed our bounds in the branch-and-bound framework of Buchheim et al. [2012a]. We compare the bounds among themselves and with the lower bound originally used by Buchheim et al. [2012a]: this is the best bound we can get if we allow for $Q^{\prime}$ only matrices of the form $Q^{\prime}=e_{i} e_{i}^{t}$, where $e_{i}$ is the $i$ th unit vector. These matrices describe splits and hence we call this bound $l b_{s p l i t}$. The matrix $Q_{s p l i t}^{\prime}$ as well as the matrices $Q_{a c}^{\prime}$ and $Q_{w c}^{\prime}$, i.e., the solutions of (5.11) and (5.9) are computed in the preprocessing phase: one matrix $Q_{d}^{\prime}$ for each depth $d$. In each node we then compute the corresponding bound $q(\bar{x})+(\bar{x}-\lfloor\bar{x}\rceil)^{t} Q_{d}^{\prime}(\bar{x}-\lfloor\bar{x}\rceil)$. On the other hand for the bounds $l b_{a p}, l b_{h q r}$ and $l b_{h q a p}$ we compute the matrix $Q^{\prime}$ in each node, since it depends not only on $Q_{d}$ but also on $\bar{x}$. For the heuristic quasi-axisparallel lower bounds we only consider $l b_{\text {hqap }}\left(t^{w c}\right)$ in the experiments, since we will see that computing $t^{a p}$ is time-consuming so that it is not practicable to use as an input.

To compare the bounds we did the following experiments: for each fixed number of variables $n$ we generated 100 instances at random:

- we generate $n$ eigenvalues of $Q$ uniformly at random from the interval $\left[10^{-4}, 1\right]$

- and $n$ vectors with uniform entries in $[-1,1]$ which we then orthonormalize and use as eigenvectors of $Q$

- the coordinates of $L$ are again chosen uniformly from the interval $[-1,1]$.

All experiments were carried out on a standard laptop with an Intel Core2 Duo processor at $2.13 \mathrm{GHz}$ RAM.

The first comparison is regarding the lower bounds provided: we compare the average relative gaps closed $\frac{l b-q(\bar{x})}{q\left(x^{*}\right)-q(\bar{x})}$, i.e., the quantity we also used to do our worst-case and average-case analysis. The results can be seen in Figure 5.12.

We have to mention first of all that we are only able to compute $l b_{a p}$ for $n \leq 30$ in reasonable time. But for these smaller instances it clearly outperforms all other approaches in this comparison. By the theoretical hierarchy we developed before it is clear that it has to be better than $l b_{a c}$ and $l b_{w c}$, but it is noteworthy that it is much better than the heuristic quasi-round and quasi-axisparallel bounds. Next we observe that using the average case gives significantly better bounds than using the worst case best underestimator, which was also to be expected as an average behavior. Furthermore, we knew before that $l b_{h q a p}\left(t^{w c}\right)$ has to be better than $l b_{w c}$, since it uses the latter one as input, but we observe that the difference becomes smaller for growing $n$ which is due to the fact that in general $\beta(t, \bar{x})$ is going to 0 for growing $n$. Comparing the two heuristic approaches it seems that the quasi-axisparallel bound is stronger than the quasi-round bound, which would also have been the case if we were able to compute the best bounds in both cases. It remains to be mentioned that the results obtained by the splits were by far worse than all our bounds and that we observe that the general ranking between the bounds seems to be independent of the dimension $n$.

But of course the main criterion to compare the different bounds has to be their performance in the branch-and-bound-framework, i.e., the most important comparison is regarding the total 


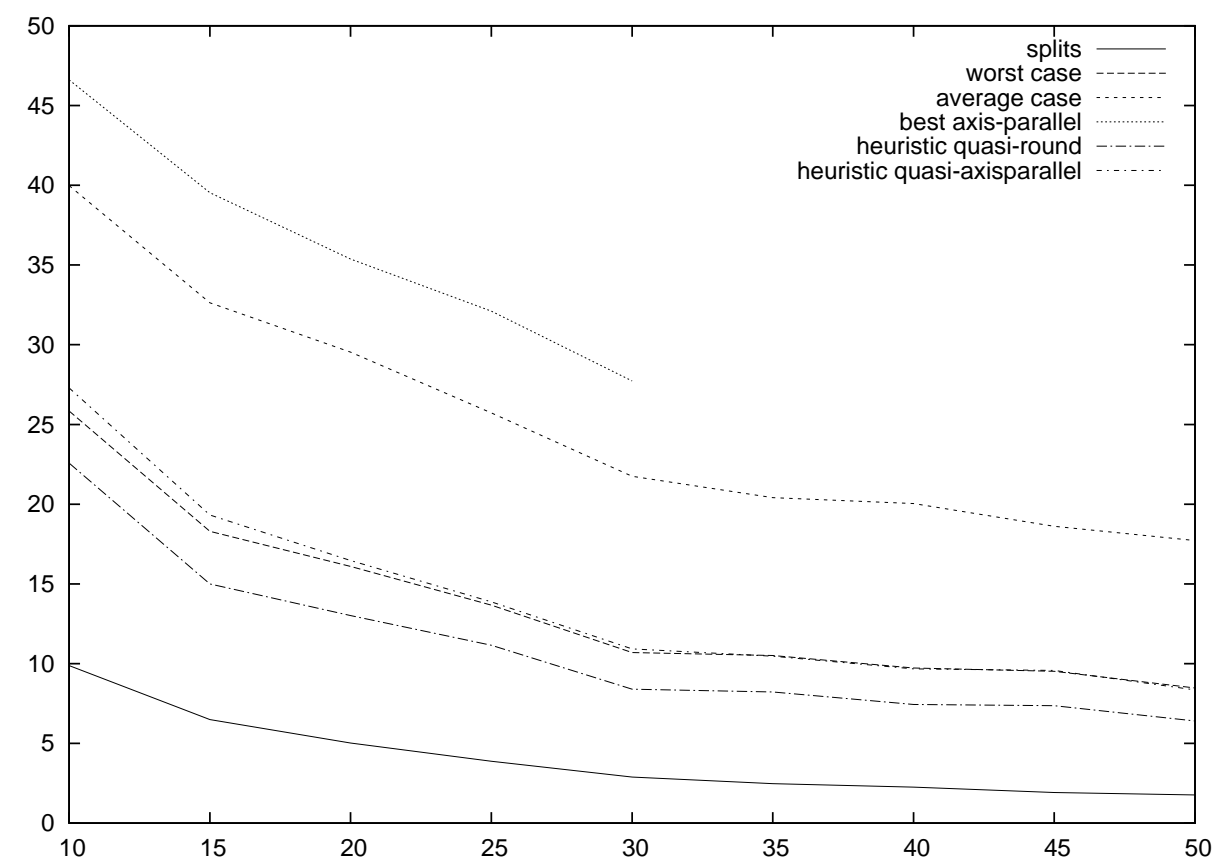

Figure 5.12.: Average relative gap closed (in \%) by number of variables.

computation time to solve a given convex quadratic integer program. This time depends of course on the quality of the bounds, since a better bound means less nodes to be enumerated, but also the time to compute the bounds has to be taken into account. We report the total runtime in Figure 5.13.

Here we observe that the heuristic quasi-round approach outperforms all others. The next best are the splits. These are the two bounds where the matrix $Q^{\prime}$ is calculated in a relatively simple and therefore computationally cheap way and where we do not need any preprocessing. Compared to the splits the heuristic quasi-round approach calculates a new matrix $Q^{\prime}$ in each node. The next best bounds are the ones that are mainly computed in the preprocessing: $l b_{a c}$ and $l b_{w c}$. We see that even though their computation in each node is faster than for the heuristic quasi-round approach and the bounds were better (compare again Figure 5.12) they do not make up for the longer preprocessing phase. Among these two we observe that again the average-case outperforms the worst-case. The heuristic quasi-axisparallel line is not to distinguish from the worst-case line in Figure 5.13: obviously it does not pay to do this readapting in each node. And the clear loser in this comparison is the best axisparallel approach. Even though the bounds are really good they are not good enough to make up for their long computation time. It seems to be better to use the approaches worst-case and average-case that compute one axisparallel ellipsoid for each node. Of course we have to say that the runtimes depend highly on the implementation. For example one could try to speed up the SDP-solving.

Summarizing, we see that there is a reason to consider different bounds as we did in this chapter since we cannot determine a theoretical dominating hierarchy between them and in the computational results they perform very different depending on whether we compare the 


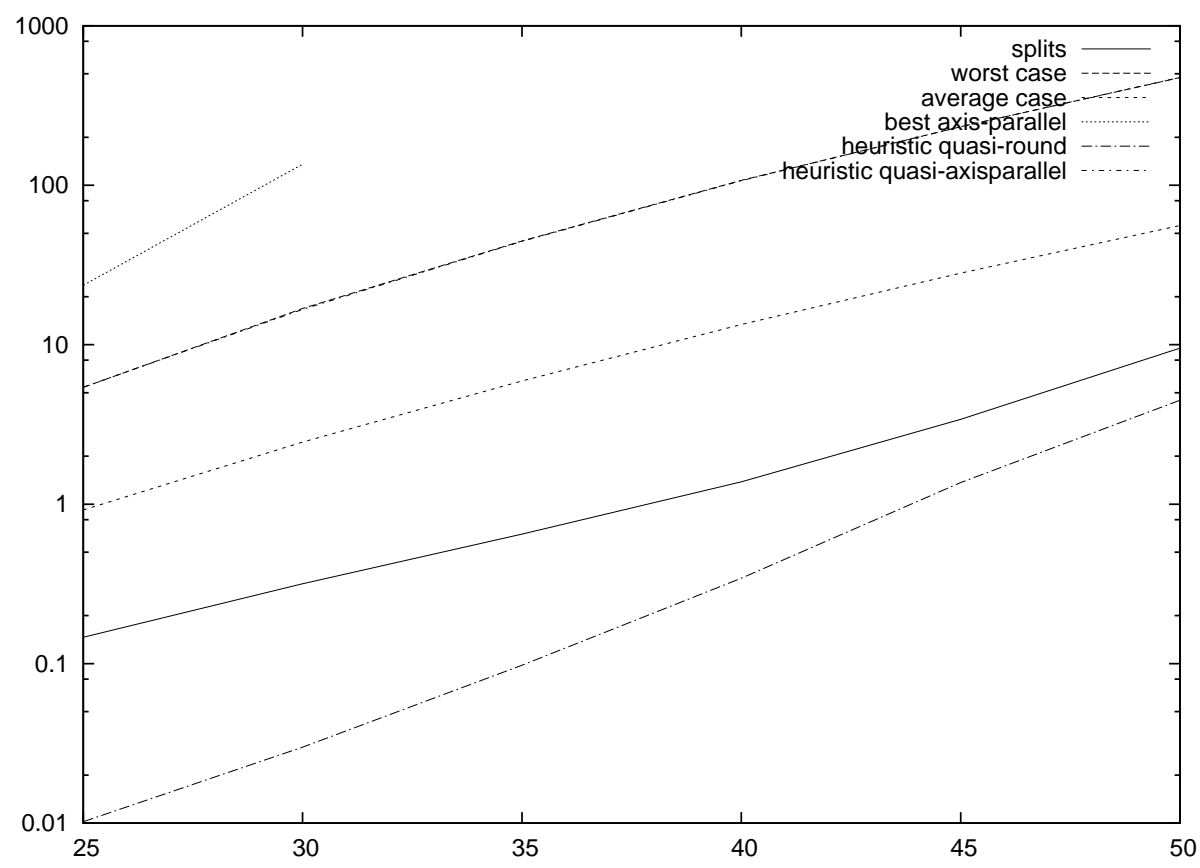

Figure 5.13.: Average total runtimes (in seconds, log scale) by number of variables.

resulting bounds or the total runtime. Compared to Buchheim et al. [2012a] where the general approach is taken from, we observe that all our bounds are tighter and that the heuristic quasiround approach also outperforms their approach in terms of total runtime. 


\subsection{Summary}

In this chapter we used the Rounding Property to solve unconstrained convex quadratic integer programs. To this end we used the fact that the level sets are ellipsoids whose shape is determined solely by the Hessian of the objective function and that the unique continuous minimizer is given by a closed formula. We proved that the level sets are cross-shaped if and only if the objective function is separable, i.e., if the level sets are axisparallel ellipsoids. Whether or not they are quasi-round for a given $\alpha$ only depends on the smallest and biggest eigenvalues. Since the level sets considered here are all bounded and fully dimensional they are all quasi-round if we allow the difference in the radii to be large enough. This enables us to give a finite candidate set for every unconstrained convex quadratic integer program.

We adapted the definition of quasi-cross-shapedness by introducing the concept of quasiaxisparallelity: a set is said to be quasi-axisparallel if there exists an axisparallel ellipsoid to whom the set is $\epsilon$-similar. As in the quasi-round case we were able to explicitly calculate the corresponding $\epsilon$ that guarantees the (Strong) Rounding Property.

If the given convex quadratic integer problem does not have the Rounding Property we use our concepts to derive lower bounds to use in a branch-and-bound algorithm. The idea is that any convex quadratic function that has the same continuous minimizer as the objective function and whose level sets contain the original level sets for each level, is a global underestimator. If we use only such underestimators that have the Strong Rounding Property, the corresponding lower bound is easy to calculate. Since we would like to have the tightest bound this means we try to find the underestimator that gives the highest lower bound and has the Strong Rounding Property. We cannot solve the problem in this generality and therefore we consider restricted versions: in problem (5.3) we look for the separable underestimator giving the tightest lower bound, in (5.4) we look for the tightest lower bound we can get by an underestimator whose level sets are $\alpha_{2}(\bar{x})$-quasi-round and so on. In contrast to (5.3) which is a SDP, we do not know how to solve (5.4). Therefore we use a heuristic: we fix the eigenvectors to be the same as the ones of the Hessian of the original problem and allow only for changes in the eigenvalues. The same heuristic can be applied to find an underestimator with quasi-axisparallel level sets if we fix first the axisparallel ellipsoid to whom it should be similar.

If we want to use these lower bounds in a branch-and-bound framework it might be a good idea to develop bounds that do not depend on the continuous minimizer, but only on the Hessian of the objective function. We obtained problems (5.9) and (5.11) by performing a worst-case and an average-case analysis.

In the last section we compared the different bounds we developed. First of all we investigated the theoretical hierarchy, compare Figure 5.10. Next we showed in an example what the underestimators look like, that they do in fact vary highly if we only translate the problem and last but not least that the theoretical hierarchy shown in Figure 5.10 is complete in the sense that for all other pairs of bounds there exist examples where either of these bounds is tighter. Nevertheless, this need not mean that there is not an average dominance between some of them. To investigate this question we showed some first computational results, indicating for example that on the one hand the separable underestimators give the tightest bounds, but that on the other hand they are too time-consuming to calculate. Comparing our bounds with the bound originally used by Buchheim et al. [2012a], in whose framework we embedded our bounds, we see that all our bounds are tighter and that one of them is able to outperform the original bound also in total runtime of the whole branch-and-bound algorithm. 


\section{Extension to mixed-integer problems}

So far our approach relied heavily on the fact that we considered pure integer problems, since like this we were able to construct our finite candidate set $\operatorname{Round}(\bar{x}) \cap F$. This raises the question whether it is possible to carry our results over to the case of mixed-integer optimization problems.

This chapter is organized as follows. First we give the general setting for the mixed-integer case and define the appropriate versions of the Rounding Property and the Strong Rounding Property. Then we investigate how we can use our results for the pure integer case to solve mixed-integer problems. In the next section we go the other direction: given a pure integer problem we consider a mixed-integer relaxation to derive a lower bound. And in the last section we apply the results to the special case of convex quadratic problems as considered in Chapter 5 .

\subsection{The mixed-integer case}

Consider an arbitrary mixed-integer problem

$$
\begin{array}{ll}
(M I P) \min & f(v, w) \\
\text { s.t. } & (v, w) \in F \\
& v \in \mathbb{Z}^{n_{1}} \\
& w \in \mathbb{R}^{n_{2}}
\end{array}
$$

where $F \subseteq \mathbb{R}^{n_{1}+n_{2}}$ and $f: F \rightarrow \mathbb{R}$.

We transfer the notation for integer problems to the case of mixed-integer problems.

Notation 6.1. We denote the set of optimal solutions to $(M I P)$ by

$$
(V, W)^{*}:=\left\{(v, w) \in F, v \in \mathbb{Z}^{n_{1}}: f(v, w) \leq f\left(v^{\prime}, w^{\prime}\right) \forall\left(v^{\prime}, w^{\prime}\right) \in F, v^{\prime} \in \mathbb{Z}^{n_{1}}\right\}
$$

and an optimal solution, i.e., a mixed-integer minimizer by $\left(v^{*}, w^{*}\right)$. 
Again we always assume that $(V, W)^{*} \neq \emptyset$ and consider the continuous relaxation

$$
\begin{array}{lll}
(C P) \min & f(v, w) \\
\text { s.t. } & (v, w) \in F \\
& (v, w) \in \mathbb{R}^{n_{1}+n_{2}} .
\end{array}
$$

Notation 6.2. We denote the set of optimal solutions to $(C P)$ by

$$
\overline{(V, W)}:=\left\{(v, w) \in F: f(v, w) \leq f\left(v^{\prime}, w^{\prime}\right) \forall\left(v^{\prime}, w^{\prime}\right) \in F\right\}
$$

and an optimal solution (a continuous minimizer) by $(\bar{v}, \bar{w})$.

We need to adapt Basic Assumption 1.5 to this case. Therefore we introduce the following notation:

Notation 6.3. (i) Denote by $\operatorname{Proj}(M)_{m}$ the projection of the set $M \subseteq \mathbb{R}^{n}$ where $n>m$ onto the first $m$ dimensions:

$$
\operatorname{Proj}(M)_{m}:=\left\{v \in \mathbb{R}^{m}: \exists w \in \mathbb{R}^{n-m}:(v, w) \in M\right\} .
$$

(ii) Given a fixed $\hat{v} \in \mathbb{R}^{n_{1}}$ and $F \subseteq \mathbb{R}^{n_{1}+n_{2}}$ we define

$$
F(\hat{v}):=\left\{w \in \mathbb{R}^{n_{2}}:(\hat{v}, w) \in F\right\} .
$$

Furthermore, for $f: F \rightarrow \mathbb{R}$ we define $f_{\hat{v}}: F(\hat{v}) \rightarrow \mathbb{R}, w \mapsto f(\hat{v}, w)$.

Basic Assumption 6.4. We always assume that $\overline{(V, W)} \neq \emptyset$ and that there exists an efficient algorithm to solve $(C P)$. This algorithm is used as a black box in the following. Furthermore, we assume that there exists an efficient algorithm to solve $\min \left\{f_{\hat{v}}(w): w \in\right.$ $F(\hat{v})\}$ for any fixed $\hat{v} \in \mathbb{Z}^{n_{1}} \cap \operatorname{Proj}(F)_{n_{1}}$.

Now we can introduce the Rounding Property for mixed-integer problems.

Definition 6.5. We say that a mixed-integer problem has the Rounding Property if for any optimal solution $(\bar{v}, \bar{w}) \in \overline{(V, W)}$ to its continuous relaxation $(C P)$ there exists an optimal solution $\left(v^{*}, w^{*}\right) \in(V, W)^{*}$ such that $v^{*} \in \operatorname{Round}(\bar{v})$.

Note here that we do not assume anything about the continuous variables $\bar{w}$ and $w^{*}$. This means also that we do neither get a finite candidate set nor any proximity result, in contrast to the pure integer case. But we get again an algorithm to solve a mixed-integer problem having the Rounding Property.

Algorithm 3: Basic algorithm to solve a mixed-integer problem that has the Rounding Property

Input: $(f, F)$ having the Rounding Property

Output: an optimal solution $\left(v^{*}, w^{*}\right) \in(V, W)^{*}$

1. solve $(C P)$ to get an optimal solution $(\bar{v}, \bar{w}) \in \overline{(V, W)}$

2. for every $\hat{v} \in \operatorname{Round}(\bar{v}) \cap \operatorname{Proj}(F)_{n_{1}}$ solve $\min \left\{f_{\hat{v}}(w): w \in F(\hat{v})\right\}$

3. Choose $\left(v^{*}, w^{*}\right) \in \operatorname{argmin}\left\{f\left(\hat{v}, w_{\hat{v}}\right): \hat{v} \in \operatorname{Round}(\bar{v}) \cap \operatorname{Proj}(F)_{n_{1}}, w_{\hat{v}} \in\right.$ $\left.\operatorname{argmin}\left\{f_{\hat{v}}(w): w \in F(\hat{v})\right\}\right\}$. 
Lemma 6.6. Algorithm 3 is well-defined and correct, i.e., for all $(\bar{v}, \bar{w}) \in \overline{(V, W)}$ we have

(i) $\operatorname{Round}(\bar{v}) \cap \operatorname{Proj}(F)_{n_{1}} \neq \emptyset$ and

(ii) the output $\left(v^{*}, w^{*}\right)$ fulfills $\left(v^{*}, w^{*}\right) \in(V, W)^{*}$.

Proof. Let $(\bar{v}, \bar{w}) \in \overline{(V, W)}$ be any optimal solution to $(C P)$. As $(M I P)$ has the RP we know that there exists $(\widetilde{v}, \widetilde{w}) \in(V, W)^{*} \subseteq F$ such that $\widetilde{v} \in \operatorname{Round}(\bar{v})$.

(i) This means $\widetilde{v} \in \operatorname{Round}(\bar{v}) \cap \operatorname{Proj}(F)_{n_{1}}$.

(ii) Let $\left(v^{*}, w^{*}\right) \in \operatorname{argmin}\left\{f\left(\hat{v}, w_{\hat{v}}\right): \hat{v} \in \operatorname{Round}(\bar{v}) \cap \operatorname{Proj}(F)_{n_{1}}, w_{\hat{v}} \in \operatorname{argmin}\left\{f_{\hat{v}}(w): w \in\right.\right.$ $F(\hat{v})\}\}$. Since $(\widetilde{v}, \widetilde{w}) \in(V, W)^{*}$ we have $\widetilde{w} \in \operatorname{argmin}\left\{f_{\widetilde{v}}(w): w \in F(\widetilde{v})\right\}:$

Assume not. Since $(\widetilde{v}, \widetilde{w}) \in F$ this means that there exists $u \in F(\widetilde{v})$ and $f(\widetilde{v}, u)<f(\widetilde{v}, \widetilde{w})$ which is a contradiction to $(\widetilde{v}, \widetilde{w}) \in(V, W)^{*}$.

Therefore we have $(\widetilde{v}, \widetilde{w}) \in \operatorname{argmin}\left\{f\left(\hat{v}, w_{\hat{v}}\right): \quad \hat{v} \in \operatorname{Round}(\bar{v}) \cap \operatorname{Proj}(F)_{n_{1}}, w_{\hat{v}} \in\right.$ $\left.\operatorname{argmin}\left\{f_{\hat{v}}(w): w \in F(\hat{v})\right\}\right\}:$

Assume not. Then there exist $v^{\prime}, w^{\prime}: v^{\prime} \in \operatorname{Round}(\bar{v}) \cap \operatorname{Proj}(F)_{n_{1}}, w^{\prime} \in$ $\operatorname{argmin}\left\{f_{v^{\prime}}(w): w \in F\left(v^{\prime}\right)\right\}$, i.e., $\left(v^{\prime}, w^{\prime}\right) \in F$ and $f\left(v^{\prime}, w^{\prime}\right)<f(\widetilde{v}, \widetilde{w})$ which is again a contradiction to $(\widetilde{v}, \widetilde{w}) \in(V, W)^{*}$.

Together we have

$$
\begin{aligned}
f\left(v^{*}, w^{*}\right)=\min \left\{f\left(\hat{v}, w_{\hat{v}}\right): \hat{v} \in \operatorname{Round}(\bar{v}) \cap \operatorname{Proj}(F)_{n_{1}},\right. & \\
\left.w_{\hat{v}} \in \operatorname{argmin}\left\{f_{\hat{v}}(w): w \in F(\hat{v})\right\}\right\} & =f(\widetilde{v}, \widetilde{w}) .
\end{aligned}
$$

Furthermore, we have $\left(v^{*}, w^{*}\right) \in F$ as $v^{*} \in \operatorname{Proj}(F)_{n_{1}}$ and $w^{*} \in F\left(v^{*}\right)$ and thus $\left(v^{*}, w^{*}\right) \in(V, W)^{*}$.

Note that this algorithm means that we are able to derive a finite candidate set by choosing an appropriate $w$ for each $v \in \operatorname{Round}(\bar{v}) \cap \operatorname{Proj}(F)_{n_{1}}$.

What does a level set approach for the mixed-integer case look like? Consider Figure 6.1:

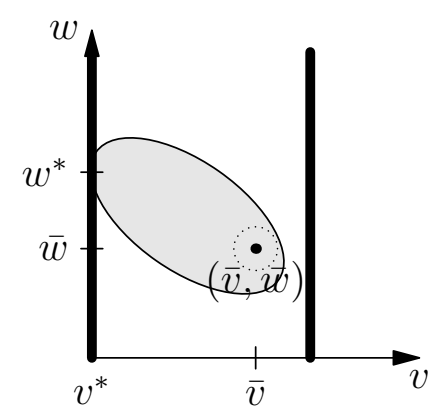

Figure 6.1.: A level set approach for the mixed-integer setting.

now we grow the level sets until they reach the first of the vertical lines representing $\mathbb{Z} \times \mathbb{R}$. In higher dimensions we get flats: consider for example the case $n=3$. Here we have the following possibilities: 
- $n_{1}=3, n_{2}=0$ : this is the pure integer case.

- $n_{1}=2, n_{2}=1$ : the feasible set is the union of all flats $\left(v_{1}, v_{2}, \mathbb{R}\right)$ for $\left(v_{1}, v_{2}\right) \in \mathbb{Z}^{2}$ as depicted in Figure 6.2 left.

- $n_{1}=1, n_{2}=2$ : the feasible set is the union of all hyperplanes $(v, \mathbb{R}, \mathbb{R})$ for $v \in \mathbb{Z}$ as depicted in Figure 6.2 right.

- $n_{1}=0, n_{2}=3$ : this is the continuous case.
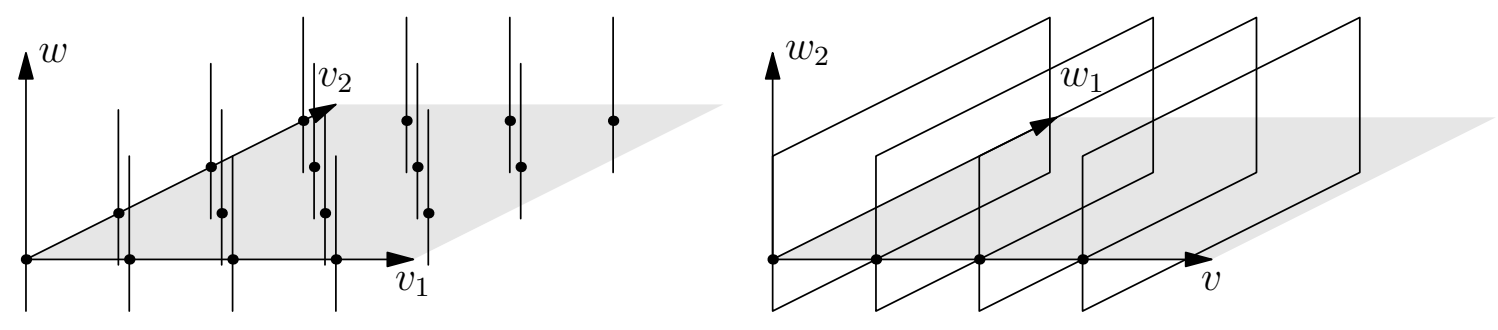

Figure 6.2.: The feasible sets for $n_{1}=2, n_{2}=1$ and $n_{1}=1, n_{2}=2$.

Basic Assumption 6.4 makes sure that Algorithm 3 is practicable: in the first step we use the black box algorithm to solve $(C P)$ which we assumed to be efficient and in the second step we use at most $2^{n}$ times the algorithm to solve $\min \left\{f_{\hat{v}}(w): w \in F(\hat{v})\right\}$ which we also assumed to be efficient. Nevertheless, the second step might take much longer than the second step in Algorithm 1 where we "just" evaluated the objective function for each $\hat{x} \in \operatorname{Round}(\bar{x})$. This means that in the case of a mixed-integer problem the Strong Rounding Property might be even more useful than in the pure integer case.

Definition 6.7. We say that a mixed-integer problem has the Strong Rounding Property if for any optimal solution $(\bar{v}, \bar{w}) \in \overline{(V, W)}$ to $(C P)$ there exists an optimal solution $\left(v^{*}, w^{*}\right) \in$ $(V, W)^{*}$ such that $v^{*}=\lfloor\bar{v}\rceil$.

Again, we do not get any proximity result out of this property. Just as in the pure integer case, we get that the Strong Rounding Property implies the Rounding Property.

Corollary 6.8. If a mixed-integer problem has the Strong Rounding Property it also has the Rounding Property.

The straightforward algorithm looks as follows.

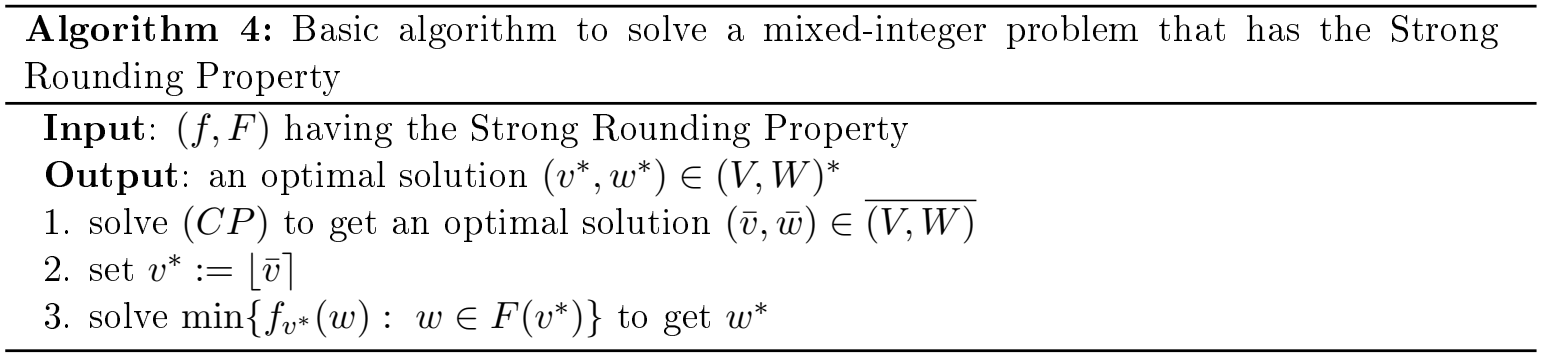

Lemma 6.9. Algorithm \& is well-defined and correct, i.e., 
(i) $v^{*} \in \operatorname{Proj}(F)_{n_{1}}$ and

(ii) $\left(v^{*}, w^{*}\right) \in(V, W)^{*}$.

Proof. Let $(\bar{v}, \bar{w}) \in \overline{(V, W)}$. As $(M I P)$ has the SRP there exists $(\widetilde{v}, \widetilde{w}) \in(V, W)^{*}$ such that $\widetilde{v}=\lfloor\bar{v}\rceil=v^{*}$. This means,

(i) $v^{*} \in \operatorname{Proj}(F)_{n_{1}}$.

(ii) $\widetilde{w} \in \operatorname{argmin}\left\{f_{v^{*}}(w): w \in F\left(v^{*}\right)\right\}:$

Assume not. Then there exists $u \in F\left(v^{*}\right)$ and $f\left(v^{*}, u\right)<f\left(v^{*}, \widetilde{w}\right)=f(\widetilde{v}, \widetilde{w})$ which is a contradiction to $(\widetilde{v}, \widetilde{w}) \in(V, W)^{*}$.

This means that

$$
f(\widetilde{v}, \widetilde{w})=f\left(v^{*}, \widetilde{w}\right)=f_{v^{*}}(\widetilde{w})=\min \left\{f_{v^{*}}(w): w \in F\left(v^{*}\right)\right\}=f_{v^{*}}\left(w^{*}\right)=f\left(v^{*}, w^{*}\right) .
$$

Since furthermore $\left(v^{*}, w^{*}\right) \in F$ we get $\left(v^{*}, w^{*}\right) \in(V, W)^{*}$.

Remark 6.10. We assume again w.l.o.g. that $\overline{(V, W)} \cap\left(\mathbb{Z}^{n_{1}} \times \mathbb{R}^{n_{2}}\right)=\emptyset$ since all $(\bar{v}, \bar{w}) \in$ $\overline{(V, W)}$ with $\bar{v} \in \mathbb{Z}^{n_{1}}$ do not impose any further constraints and can therefore be ignored. 


\subsection{Connection to the pure integer case}

A natural question is whether the (Strong) Rounding Property for mixed-integer problems is somehow connected to the (Strong) Rounding Property for integer problems. This question is investigated in this section. Given $(M I P)$ as in (6.1) we consider its pure integer counterpart

$$
\begin{array}{lll}
(I P) & \min & f(x) \\
\text { s.t. } & x \in F \\
& x \in \mathbb{Z}^{n_{1}+n_{2}} .
\end{array}
$$

We observe that in general there is no connection between $(M I P)$ having the (Strong) Rounding Property and $(I P)$ having the (Strong) Rounding Property: it is possible that $(I P)$ has the Strong Rounding Property and $(M I P)$ does not have the Rounding Property and vice versa:

Example 6.11. (i) (IP) has the Strong Rounding Property, but (MIP) does not have the Rounding Property (no matter if $v$ or $w$ has to be integer): consider the following objective function (compare Figure 6.3)

$$
f(v, w)= \begin{cases}1 & \text { if }(v, w)=(\bar{v}, \bar{w}) \\ 2 & \text { if }(v, w) \in \text { dark gray area } \\ 3 & \text { if }(v, w) \in \text { light gray area } \\ 4 & \text { else }\end{cases}
$$

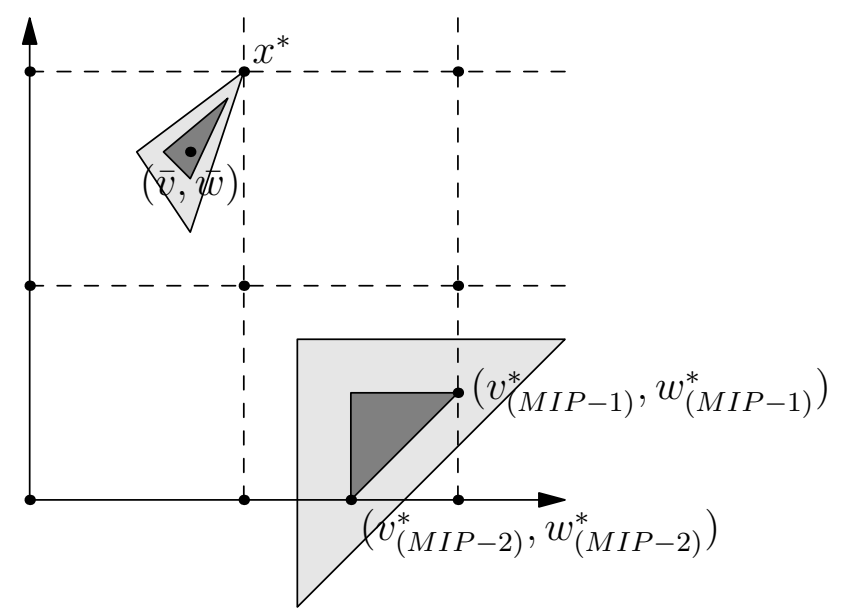

Figure 6.3.: The areas defining $f(v, w)$ and therefore also the level sets $\mathcal{L}_{\leq}(1), \mathcal{L}_{\leq}(2)$ and $\mathcal{L}_{\leq}(3)$.

We see that $(\bar{v}, \bar{w})$ is the unique optimal solution to the shared continuous relaxation of both $(I P)$ and $(M I P)$. In addition we see that (IP) has the Strong Rounding Property. In contrast neither

$$
\begin{array}{ccc}
(M I P-1) & \min & f(v, w) \\
\text { s.t. } & v \in \mathbb{Z} \\
& w \in \mathbb{R}
\end{array}
$$


nor

$$
\begin{array}{ccl}
(M I P-2) & \min & f(v, w) \\
\text { s.t. } & v \in \mathbb{R} \\
& w \in \mathbb{Z}
\end{array}
$$

has the Rounding Property.

(ii) On the other hand it is also possible that (MIP) has the Strong Rounding Property but (IP) does not have the Rounding Property:

consider again the following objective function, where the areas are this time as in Figure 6.4:

$$
f(v, w)= \begin{cases}1 & \text { if }(v, w)=(\bar{v}, \bar{w}) \\ 2 & \text { if }(v, w) \in \text { dark gray area } \\ 3 & \text { if }(v, w) \in \text { light gray area } \\ 4 & \text { else }\end{cases}
$$

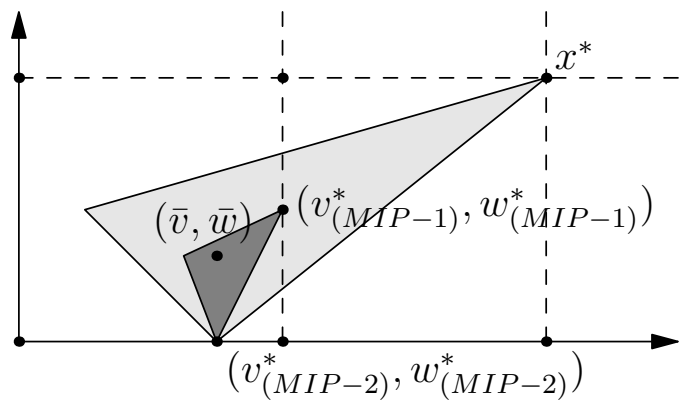

Figure 6.4.: The areas defining $f(v, w)$ and therefore also the level sets $\mathcal{L}_{\leq}(1), \mathcal{L}_{\leq}(2)$ and $\mathcal{L}_{\leq}(3)$.

We observe that $(M I P-1)$ as well as $(M I P-2)$ have the Strong Rounding Property whereas $(I P)$ does not have the Rounding Property.

Note that these examples do not depend on the fact that $n_{1}+n_{2}=2$, but can easily be carried over to higher dimensions.

Of course it is not true that the Rounding Property for mixed-integer problems is completely independent of the Rounding Property for pure integer problems, but the pure integer counterpart is not the "right" integer problem to consider. In the following we establish the connection between the (Strong) Rounding Property for a mixed-integer problem and the (Strong) Rounding Property for a (lower dimensional) pure integer problem.

Notation 6.12. (i) Define $g: \operatorname{Proj}(F)_{n_{1}} \rightarrow \mathbb{R}$ by $g(v):=\min \left\{f_{v}(w): w \in F(v)\right\}$.

(ii) Denote by $(I P)_{g}$ the pure integer problem

$$
\begin{array}{rrl}
(I P)_{g} & \min & g(v) \\
& \text { s.t. } & v \in \operatorname{Proj}(F)_{n_{1}} \\
& v \in \mathbb{Z}^{n_{1}}
\end{array}
$$


and the set of optimal solutions to $(I P)_{g}$ by $V_{g}^{*}$.

(iii) Denote by $(C P)_{g}$ the corresponding continuous relaxation

$$
\begin{array}{rcl}
(C P)_{g} & \min & g(v) \\
& \text { s.t. } & v \in \operatorname{Proj}(F)_{n_{1}}
\end{array}
$$

and the set of optimal solutions to $(C P)_{g}$ by $\bar{V}_{g}$.

We assume that these notations are well-defined:

Basic Assumption 6.13. In the following we assume not only that $(V, W)^{*} \neq \emptyset$ and $\overline{(V, W)} \neq \emptyset$ but also that $g(v)$ exists for all $v \in \operatorname{Proj}(F)_{n_{1}}$.

This can for example be guaranteed by assuming that $f(v, \cdot)$ is continuous in $w$ for any fixed $v$ and that $F$ is compact:

Let $v \in \operatorname{Proj}(F)_{n_{1}}$. Due to our assumption $f(v, w)$ is continuous in $w$. Since $v \in \operatorname{Proj}(F)_{n_{1}}$ we know that $F(v) \neq \emptyset$ and furthermore since $F$ is compact also $F(v)$ is compact as it is the intersection of the compact set $F$ with a $n_{2}$ dimensional flat. This means that $g(v):=\min \left\{f_{v}(w): w \in F(v)\right\}$ exists due to the extreme value theorem of Weierstrass and hence $\operatorname{argmin}\left\{f_{v}(w): w \in F(v)\right\} \neq \emptyset$ for all $v \in \operatorname{Proj}(F)_{n_{1}}$.

The following theorem establishes the connection between the sets of optimal solutions to $(M I P)$ and $(I P)_{g}$ :

Theorem 6.14. (i) $\operatorname{Proj}(\overline{(V, W)})_{n_{1}}=\bar{V}_{g}$ and

(ii) $\operatorname{Proj}\left((V, W)^{*}\right)_{n_{1}}=V_{g}^{*}$.

Proof. Ad (i)

"ᄃ" Let $\bar{v} \in \operatorname{Proj}(\overline{(V, W)})_{n_{1}}$, i.e., there exists $\bar{w} \in F(\bar{v})$ such that $(\bar{v}, \bar{w}) \in \overline{(V, W)}$. Assume that $\bar{v} \notin \bar{V}_{g}$. Since $\bar{v} \in \operatorname{Proj}(F)_{n_{1}}$ this means that there exists $u \in \operatorname{Proj}(F)_{n_{1}}$ s.t.

$$
\min _{w \in F(u)} f_{u}(w)=g(u)<g(\bar{v})=\min _{w \in F(\bar{v})} f_{\bar{v}}(w) \leq f(\bar{v}, \bar{w})
$$

which means that there exists $w(u) \in F(u)$ such that $f(u, w(u))<f(\bar{v}, \bar{w})$ and is hence a contradiction to $(\bar{v}, \bar{w}) \in \overline{(V, W)}$. Hence $\bar{v} \in \bar{V}_{g}$.

"卫" Let $\bar{v}_{g} \in \bar{V}_{g} \subseteq \operatorname{Proj}(F)_{n_{1}}$. This means, there exists $\bar{w}_{g} \in \operatorname{argmin}\left\{f_{\bar{v}_{g}}(w): w \in F\left(\bar{v}_{g}\right)\right\}$. Assume that $\left(\bar{v}_{g}, \bar{w}_{g}\right) \notin \overline{(V, W)}$. Since $\left(\bar{v}_{g}, \bar{w}_{g}\right) \in F$ this means that there exists $(u, t) \in F$ such that $f(u, t)<f\left(\bar{v}_{g}, \bar{w}_{g}\right)$ which means

$$
g(u) \leq f(u, t)<f\left(\bar{v}_{g}, \bar{w}_{g}\right)=g\left(\bar{v}_{g}\right)
$$

and is hence a contradiction to $\bar{v}_{g} \in \bar{V}_{g}$. Thus $\left(\bar{v}_{g}, \bar{w}_{g}\right) \in \overline{(V, W)}$ and therefore $\bar{v}_{g} \in$ $\operatorname{Proj}(\overline{(V, W)})_{n_{1}}$.

(ii) follows analogously. 
Using this theorem we can show the connection between the (Strong) Rounding Property for $(M I P)$ and for $(I P)_{g}$.

Corollary 6.15. (MIP) has the (Strong) Rounding Property if and only if $(I P)_{g}$ has the (Strong) Rounding Property.

Proof. Using Theorem 6.14 shows:

" $\Rightarrow$ " Let $\bar{v}_{g} \in \bar{V}_{g}=\operatorname{Proj}(\overline{(V, W)})_{n_{1}}$. This means that there exists $\bar{w}_{g} \in F\left(\bar{v}_{g}\right)$ s.t. $\left(\bar{v}_{g}, \bar{w}_{g}\right) \in$ $\overline{(V, W)}$. Since $(M I P)$ has the (Strong) Rounding Property there exists $\left(v^{*}, w^{*}\right) \in$ $(V, W)^{*}$ such that $v^{*} \in \operatorname{Round}\left(\bar{v}_{g}\right)$ (or $v^{*}=\left\lfloor\bar{v}_{g}\right\rceil$ respectively).

Since $v^{*} \in \operatorname{Proj}\left((V, W)^{*}\right)_{n_{1}}=V_{g}^{*}$ this means that $(I P)_{g}$ has the (Strong) Rounding Property.

"६" Let $(\bar{v}, \bar{w}) \in \overline{(V, W)}$. This means that $\bar{v} \in \operatorname{Proj}(\overline{(V, W)})=\bar{V}_{g}$. Since $(I P)_{g}$ has the (Strong) Rounding Property this means that there exists $v_{g}^{*} \in V_{g}^{*}$ such that $v_{g}^{*} \in$ $\operatorname{Round}(\bar{v})$ (or $v_{g}^{*}=\lfloor\bar{v}\rceil$ ). As $V_{g}^{*}=\operatorname{Proj}\left((V, W)^{*}\right)_{n_{1}}$ there exists $w_{g}^{*} \in F\left(v_{g}^{*}\right)$ s.t. $\left(v_{g}^{*}, w_{g}^{*}\right) \in(V, W)^{*}$ and thus $(M I P)$ has the (Strong) Rounding Property.

Note that in addition to the claim of Theorem 6.14 we also showed how to derive an optimal solution to (6.1) and (6.2) out of optimal solutions to (6.3) and (6.4):

- Let $\bar{v}_{g} \in \bar{V}_{g}$ then $\left(\bar{v}_{g}, \bar{w}_{g}\right) \in \overline{(V, W)}$ for any $\bar{w}_{g} \in \operatorname{argmin}\left\{f_{\bar{v}_{g}}(w): w \in F\left(\bar{v}_{g}\right)\right\}$.

- Let $v_{g}^{*} \in V_{g}^{*}$ then $\left(v_{g}^{*}, w_{g}^{*}\right) \in(V, W)^{*}$ for any $w_{g}^{*} \in \operatorname{argmin}\left\{f_{v_{g}^{*}}(w): w \in F\left(v_{g}^{*}\right)\right\}$.

As in Lemma 1.18 we use a level set approach to determine whether a given mixed-integer problem has the (Strong) Rounding Property or not. Therefore we first of all investigate what the level sets of $(I P)_{g}$ look like:

$$
\begin{aligned}
\mathcal{L}_{\leq, g}^{\operatorname{Proj}(F)_{n_{1}}}(z) & =\left\{v \in \operatorname{Proj}(F)_{n_{1}}: g(v) \leq z\right\} \\
& =\left\{v \in \operatorname{Proj}(F)_{n_{1}}: \min _{w \in F(v)} f_{v}(w) \leq z\right\} \\
& =\left\{v \in \mathbb{R}^{n_{1}}: \exists w \in \mathbb{R}^{n_{2}}:(v, w) \in F \text { and } f(v, w) \leq z\right\} \\
& =\operatorname{Proj}\left(\mathcal{L}_{\leq, f}^{F}(z)\right)_{n_{1}} .
\end{aligned}
$$

Combining Corollary 6.15 and Lemma 1.18 we get the following corollary.

Corollary 6.16. $\quad-(M I P)$ has the Rounding Property $\Longleftrightarrow$ for any optimal solution $\bar{v}_{g}$ to $(C P)_{g}$ and for all $v \in \mathbb{Z}^{n_{1}} \cap \operatorname{Proj}(F)_{n_{1}}$ we have that

$$
\operatorname{Proj}\left(\mathcal{L}_{\leq, f}^{F}(g(v))\right)_{n_{1}} \cap \operatorname{Round}\left(\bar{v}_{g}\right) \neq \emptyset .
$$

- $(M I P)$ has the Strong Rounding Property $\Longleftrightarrow$ for any optimal solution $\bar{v}_{g}$ to $(C P)_{g}$ and for all $v \in \mathbb{Z}^{n_{1}} \cap \operatorname{Proj}(F)_{n_{1}}$ we have that

$$
\left\lfloor\bar{v}_{g}\right\rceil \in \operatorname{Proj}\left(\mathcal{L}_{\leq, f}^{F}(g(v))\right)_{n_{1}} .
$$


Proof. $\quad \bullet \Leftrightarrow\left(\right.$ compare (6.5)) for any optimal solution $\bar{v}_{g}$ to $(C P)_{g}$ and for all $v \in \mathbb{Z}^{n_{1}} \cap$ $\operatorname{Proj}(F)_{n_{1}}$ we have that

$$
\mathcal{L}_{\leq, g}^{\operatorname{Proj}(F)_{n_{1}}}(g(v)) \cap \operatorname{Round}\left(\bar{v}_{g}\right) \neq \emptyset .
$$

$\Leftrightarrow$ (using Lemma 1.18) $(I P)_{g}$ has the Rounding Property

$\Leftrightarrow$ (using Corollary 6.15) (MIP) has the Rounding Property.

- $\Leftrightarrow\left(\right.$ compare (6.5)) for any optimal solution $\bar{v}_{g}$ to $(C P)_{g}$ and for all $v \in \mathbb{Z}^{n_{1}} \cap \operatorname{Proj}(F)_{n_{1}}$ we have that

$$
\left\lfloor\bar{v}_{g}\right\rceil \in \mathcal{L}_{\leq, g}^{\operatorname{Proj}(F)_{n_{1}}}(g(v)) .
$$

$\Leftrightarrow$ (using Lemma 1.18) $(I P)_{g}$ has the Strong Rounding Property

$\Leftrightarrow$ (using Corollary 6.15) (MIP) has the Strong Rounding Property.

This means, if we know that the projections of the level sets of $(M I P)$ have one of the shapes identified in Part I, we know that $(M I P)$ has the Rounding Property. Hence Lemma 1.25 implies the following lemma for the case that $n_{1}=1$.

Lemma 6.17. The mixed-integer problem

$$
\begin{array}{lll}
(M I P) \min & f(v, w) \\
\text { s.t. } & (v, w) \in F \\
& v \in \mathbb{Z} \\
& w \in \mathbb{R}^{n-1}
\end{array}
$$

- has the Rounding Property if for any optimal solution $\bar{v}_{g} \in \bar{V}_{g}$ to $(C P)_{g}$ the level sets $\mathcal{L}_{\leq, f}^{F}(z)$ are connected for all levels $g\left(\bar{v}_{g}\right) \leq z \leq \min \left\{g(v): v \in \operatorname{Round}\left(\bar{v}_{g}\right) \cap \operatorname{Proj}(F)_{1}\right\}$.

- has the Strong Rounding Property if for any optimal solution $\bar{v}_{g} \in \bar{V}_{g}$ to $(C P)_{g}$ we have $\left\lfloor\bar{v}_{g}\right\rceil \in F$ and the level sets $\mathcal{L}_{\leq, f}^{F}(z)$ are connected and symmetric about $\bar{v}_{g}$ for all levels $g\left(\bar{v}_{g}\right) \leq z \leq g\left(\left\lfloor\bar{v}_{g}\right\rceil\right)$.

Proof. - Let $\bar{v}_{g} \in \bar{V}_{g}$. If the level sets $\mathcal{L}_{\leq, f}^{F}(z)$ are connected for all levels $g\left(\bar{v}_{g}\right) \leq z \leq$ $\min \left\{g(v): \quad v \in \operatorname{Round}\left(\bar{v}_{g}\right) \cap \operatorname{Proj}(F)_{1}\right\}$ also the sets $\operatorname{Proj}\left(\mathcal{L}_{\leq, f}^{F}(z)\right)_{1}$ are connected for all $g\left(\bar{v}_{g}\right) \leq z \leq \min \left\{g(v): v \in \operatorname{Round}\left(\bar{v}_{g}\right) \cap \operatorname{Proj}(F)_{1}\right\}$ and thus the level sets $\mathcal{L}_{<, g}^{\operatorname{Proj}(F)_{1}}(z)$ are connected for all $g\left(\bar{v}_{g}\right) \leq z \leq \min \left\{g(v): v \in \operatorname{Round}\left(\bar{v}_{g}\right) \cap \operatorname{Proj}(F)_{1}\right\}$. This means, compare Lemma 1.25, that $(I P)_{g}$ has the Rounding Property and hence, compare Corollary 6.15, (MIP) has the Rounding Property.

- Let $\bar{v}_{g} \in \bar{V}_{g}$. If the level sets $\mathcal{L}_{\leq, f}^{F}(z)$ are connected and symmetric about $\bar{v}_{g}$ for all level $g\left(\bar{v}_{g}\right) \leq z \leq g\left(\left\lfloor\bar{v}_{g}\right\rceil\right)$ also the sets $\operatorname{Proj}\left(\mathcal{L}_{\leq, f}^{F}(z)\right)_{1}$ are connected and symmetric about $\bar{v}_{g}$ for all $g\left(\bar{v}_{g}\right) \leq z \leq g\left(\left\lfloor\bar{v}_{g}\right\rceil\right)$. But this is equivalent to the fact that all level sets $\mathcal{L}_{\leq, g}^{\operatorname{Proj}(F)_{1}}(z)$ are symmetric intervals about $\bar{v}_{g}$ for all levels $g\left(\bar{v}_{g}\right) \leq z \leq g\left(\left\lfloor\bar{v}_{g}\right\rceil\right)$. Hence, compare Lemma 1.25, $(I P)_{g}$ has the Strong Rounding Property and thus, compare Corollary 6.15, (MIP) has the Strong Rounding Property. 


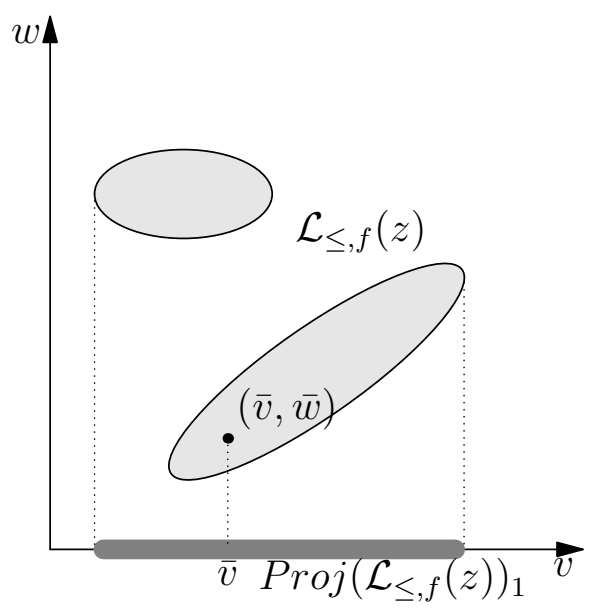

Figure 6.5.: $\operatorname{Proj}\left(\mathcal{L}_{\leq, f}(z)\right)_{1}$ is connected, even though $\mathcal{L}_{\leq, f}(z)$ is not.

Note on the one hand that connectedness is only sufficient but not necessary in Lemma 1.25 but on the other hand also that $\mathcal{L}_{\leq, f}^{F}(z)$ being connected is sufficient but not at all necessary for $\operatorname{Proj}\left(\mathcal{L}_{\leq, f}^{F}(z)\right)_{1}$ being connected, compare for example Figure 6.5.

We end this section by showing that the projection of a set is at least as round as the original set and that it is cross-shaped if the original set is.

Lemma 6.18. (i) Let $M \subseteq \mathbb{R}^{n}$ be cross-shaped w.r.t. $x^{0} \in M$. Then Proj( $\left.M\right)_{m}$ is crossshaped w.r.t. $\left(x_{1}^{0}, \ldots, x_{m}^{0}\right)$ for all $m \leq n$.

(ii) Let $M \subseteq \mathbb{R}^{n}$ fulfill $B_{p}\left(x^{0}, r\right) \subseteq M \subseteq B_{p}\left(x^{0}, R\right)$. Then we have for all $m \leq n$

$$
B_{p}\left(\left(x_{1}^{0}, \ldots, x_{m}^{0}\right), r\right) \subseteq \operatorname{Proj}(M)_{m} \subseteq B_{p}\left(\left(x_{1}^{0}, \ldots, x_{m}^{0}\right), R\right) .
$$

Proof. (i) Let $v \in \operatorname{Proj}(M)_{m}$, i.e., there exists $w \in \mathbb{R}^{n-m}$ s.t. $(v, w) \in M$. Since $M$ is cross-shaped w.r.t. $x^{0}$, this means, $\left[(v, w), x^{0}\right]_{1} \subseteq M$.

Let $p \in\left[v,\left(x_{1}^{0}, \ldots, x_{m}^{0}\right)\right]_{1}$, i.e., $p_{i}=\lambda_{i} v_{i}+\left(1-\lambda_{i}\right) x_{i}^{0}$ where $\lambda_{i} \in[0,1]$ for all $i=1, \ldots, m$. Define $q:=\left(p, x_{m+1}^{0}, \ldots, x_{n}^{0}\right)$. Then we have $q \in\left[(v, w), x^{0}\right]_{1} \subseteq M$ and therefore $p \in$ $\operatorname{Proj}(M)_{m}$.

(ii) Let $v \in B_{p}\left(\left(x_{1}^{0}, \ldots, x_{m}^{0}\right), r\right)$, i.e., $\left\|v-\left(x_{1}^{0}, \ldots, x_{m}^{0}\right)\right\|_{p} \leq r$.

This means, $\left\|\left(v, x_{m+1}^{0}, \ldots, x_{n}^{0}\right)-x^{0}\right\|_{p} \leq r$, hence $\left(v, x_{m+1}^{0}, \ldots, x_{n}^{0}\right) \in B_{p}\left(x^{0}, r\right) \subseteq M$ and like this $v \in \operatorname{Proj}(M)_{m}$.

Let $v \in \operatorname{Proj}(M)_{m}$, i.e., there exists $w \in \mathbb{R}^{n-m}$ such that $(v, w) \in M \subseteq B_{2}\left(x^{0}, R\right)$. Thus $\sum_{i=1}^{m}\left(v_{i}-x_{i}^{0}\right)^{p}+\sum_{i=m+1}^{n}\left(w_{i-m}-x_{i}^{0}\right)^{p} \leq R^{p}$ and hence $\sum_{i=1}^{m}\left(v_{i}-x_{i}^{0}\right)^{p} \leq R^{p}$ which means $v \in B_{p}\left(\left(x_{1}^{0}, \ldots, x_{m}^{0}\right), R\right)$.

For our theory this means that if $\mathcal{L}_{\leq, f}^{F}(z)$ is cross-shaped w.r.t. $\bar{x}, \mathcal{L}_{\leq, g}^{\operatorname{Proj}(F)_{n_{1}}}(z)$ is cross-shaped w.r.t. $\left(\bar{x}_{1}, \ldots, \bar{x}_{n_{1}}\right)$ and if $\mathcal{L}_{\leq, f}(z)$ is $\alpha$-quasi-round w.r.t. $\bar{x}, \mathcal{L}_{\leq, g}^{\operatorname{Proj}(F)_{n_{1}}}(z)$ is $\alpha^{\prime}$-quasi-round w.r.t. $\left(\bar{x}_{1}, \ldots, \bar{x}_{n_{1}}\right)$ for some $\alpha^{\prime} \leq \alpha$.

Since we showed that $\bar{V}_{g}=\operatorname{Proj}(\overline{(V, W)})_{n_{1}}$ we can use these results to prove the Rounding Property for $(I P)_{g}$ under conditions similar to those in Chapter 2 and Chapter 3. 
Remark 6.19. This approach can be seen as a decomposition algorithm since we solved (MIP) by reformulating it as $\min \left\{\min \left\{f_{v}(w): w \in F(v)\right\}: v \in \operatorname{Proj}(F), v \in \mathbb{Z}^{n_{1}}\right\}$. Geoffrion [1970] showed that this problem is equivalent to the original mixed-integer problem. Hence our subproblem $\min \left\{f_{v}(w): w \in F(v)\right\}$ is a continuous problem and we assume that it is efficiently solvable. In our setting $(I P)_{g}$ is easy to solve since it has the Rounding Property. If this is not the case one would use a reformulation of $(I P)_{g}$ as in an outer approximation approach or the generalized Benders decomposition, compare Section 1.1. 


\subsection{A mixed-integer relaxation to pure integer problems}

We use a mixed-integer relaxation to get a lower bound on the optimal objective value of a pure integer problem.

The idea here is the following: assume that the given integer problem

$$
\text { (IP) } \min \left\{f(x): x \in F, x \in \mathbb{Z}^{n}\right\}
$$

does not have the Rounding Property, but there exists a partition $x=(v, w)$ of the variables such that the resulting mixed-integer problem

$$
(M I P) \quad \min \left\{f(v, w):(v, w) \in F, v \in \mathbb{Z}^{n_{1}}, w \in \mathbb{R}^{n_{2}}\right\}
$$

has the (Strong) Rounding Property. Then we can use the (easy to calculate) optimal objective value of $(M I P)$ as a lower bound on the optimal objective value of $(I P)$.

Lemma 6.20. $(M I P)$ is a relaxation of $(I P)$ and hence gives a lower bound.

Proof. The feasible set of $(I P)\left\{(v, w) \in F: v \in \mathbb{Z}^{n_{1}}, w \in \mathbb{Z}^{n_{2}}\right\}$ is contained in the feasible set of $(M I P)\left\{(v, w) \in F: v \in \mathbb{Z}^{n_{1}}, w \in \mathbb{R}^{n_{2}}\right\}$ and the objective functions coincide.

This is of course only helpful if $(M I P)$ is easy to solve, which we guarantee by assuming that $(M I P)$ has the (Strong) Rounding Property, and if the bound obtained by $(M I P)$ is strictly better than the one obtained by the continuous relaxation

$$
(C P) \min \left\{f(x): x \in F, x \in \mathbb{R}^{n}\right\} .
$$

First of all we observe that $(M I P)$ and $(I P)$ have the same continuous relaxation $(C P)$. As before let $\bar{X}$ denote the set of optimal solutions to $(C P),(V, W)^{*}$ the set of optimal solutions to $(M I P)$ and $X^{*}$ the set of optimal solutions to $(I P)$. Then we have the following hierarchy of bounds.

Lemma 6.21. Let $\bar{x} \in \bar{X},\left(v^{*}, w^{*}\right) \in(V, W)^{*}$ and $x^{*} \in X^{*}$. Then we have $f(\bar{x}) \leq f\left(v^{*}, w^{*}\right) \leq$ $f\left(x^{*}\right)$. If $\bar{X} \cap\left(\mathbb{Z}^{n_{1}} \times \mathbb{R}^{n_{2}}\right)=\emptyset$ we get

$$
f(\bar{x})<f\left(v^{*}, w^{*}\right) \leq f\left(x^{*}\right) .
$$

Proof. As mentioned before $(M I P)$ is a relaxation of $(I P)$ and hence $f\left(v^{*}, w^{*}\right) \leq f\left(x^{*}\right)$. On the other hand, $(C P)$ is a relaxation of $(M I P)$ and therefore $f(\bar{x}) \leq f\left(v^{*}, w^{*}\right)$. If $\bar{X} \cap\left(\mathbb{Z}^{n_{1}} \times\right.$ $\left.\mathbb{R}^{n_{2}}\right)=\emptyset$ we have that $\left(v^{*}, w^{*}\right) \notin \bar{X}$ and hence $f\left(v^{*}, w^{*}\right)>f(\bar{x})$ since $\left(v^{*}, w^{*}\right) \in F$.

This means, if $\bar{X} \cap\left(\mathbb{Z}^{n_{1}} \times \mathbb{R}^{n_{2}}\right)=\emptyset(M I P)$ gives a strictly better bound than $(C P)$. Hence the goal is to find a partition of the variables of $(I P)$ into $v$ and $w$ variables such that the corresponding mixed-integer problem has the (Strong) Rounding Property and where no optimal solution to the continuous relaxation has integer $v$-variables.

Geometrically, this means that we look for a subspace $\mathbb{R}^{n_{1}}$ such that the projection of the level set $\mathcal{L}_{\leq, f}^{F}(z)$ onto this subspace is cross-shaped or quasi-round and the intersection of the projection of the continuous minimizer with the integer lattice $\mathbb{Z}^{n_{1}}$ is empty. An example for such a situation is given next. 
Lemma 6.22. Consider an objective function of the form

$$
f(x)=\sum_{i=1}^{k} a_{i}\left(x_{i}-b_{i}\right)^{2}+f^{\prime}\left(x_{k+1}, \ldots, x_{n}\right)
$$

where $a, b \in \mathbb{R}^{k}$ and $m:=\min _{y \in \mathbb{R}^{n-k}} f^{\prime}(y)$ exists.

Then $\operatorname{Proj}\left(\mathcal{L}_{\leq, f}(z)\right)_{k}=\sqrt{z-m} E(\operatorname{Diag}(a), b)$.

Proof. "ᄃ" Let $v \in \operatorname{Proj}\left(\mathcal{L}_{\leq, f}(z)\right)_{k}$, i.e., there exists $w \in \mathbb{R}^{n-k}$ such that $f(v, w)=$ $\sum_{i=1}^{k} a_{i}\left(v_{i}-b_{i}\right)^{2}+f^{\prime}(w) \leq z$. Then $\sum_{i=1}^{k} a_{i}\left(v_{i}-b_{i}\right)^{2} \leq z-f^{\prime}(w) \leq z-m$ and hence $v \in \sqrt{z-m} E(\operatorname{Diag}(a), b)$.

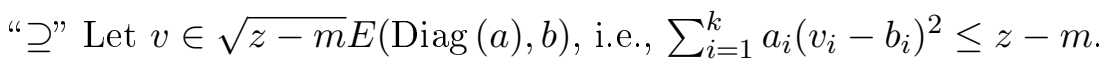

Let $w \in \operatorname{argmin}_{y \in \mathbb{R}^{n-k}} f^{\prime}(y)$, i.e., $f^{\prime}(w)=m$, hence $f(v, w)=\sum_{i=1}^{k} a_{i}\left(v_{i}-b_{i}\right)^{2}+f^{\prime}(w)=$ $\sum_{i=1}^{k} a_{i}\left(v_{i}-b_{i}\right)^{2}+m \leq z$ and therefore $v \in \operatorname{Proj}\left(\mathcal{L}_{\leq, f}(z)\right)_{k}$.

This means that in this situation the projection of the level sets are axisparallel ellipsoids about $b$. Since the objective function decomposes into two parts we know furthermore that $\operatorname{Proj}(\bar{X})_{k}=\{b\}$ and hence the resulting mixed-integer relaxation has the Strong Rounding Property. If furthermore $b \notin \mathbb{Z}$ it gives a better bound than the continuous relaxation.

We can generalize this example further as done in the next lemma.

Lemma 6.23. Consider an objective function of the form

$$
f(x)=\sum_{i=1}^{k} f_{i}\left(x_{i}\right)+f^{\prime}\left(x_{k+1}, \ldots, x_{n}\right)
$$

where $f_{i}: \mathbb{R} \rightarrow \mathbb{R}$ is quasiconvex for all $i \in\{1, \ldots, k\}$ and $m:=\min _{y \in \mathbb{R}^{n-k}} f^{\prime}(y)$ exists. Then $\operatorname{Proj}\left(\mathcal{L}_{<, f}(z)\right)_{k}$ is cross-shaped w.r.t. the projection of any continuous minimizer.

Proof. Let $\bar{x} \in \bar{X}$. This means that $f_{i}\left(\bar{x}_{i}\right) \leq f_{i}(x)$ for all $x \in \mathbb{R}$ and for all $i \in\{1, \ldots, k\}$ and $f^{\prime}\left(\bar{x}_{k+1}, \ldots, \bar{x}_{n}\right)=m$.

Let $v \in \operatorname{Proj}\left(\mathcal{L}_{\leq, f}(z)\right)_{k}$, i.e., there exists $w \in \mathbb{R}^{n-k}$ such that $f(v, w)=\sum_{i=1}^{k} f_{i}\left(v_{i}\right)+f^{\prime}(w) \leq$ $z$ and hence $\sum_{i=1}^{k} f_{i}\left(v_{i}\right) \leq z-m$.

Let $p \in\left[v,\left(\bar{x}_{1}, \ldots, \bar{x}_{k}\right)\right]_{1}$, i.e., $p_{i}=\lambda_{i} v_{i}+\left(1-\lambda_{i}\right) \bar{x}_{i}$ for $\lambda_{i} \in[0,1]$ for all $i \in\{1, \ldots, k\}$. This means,

$$
\begin{aligned}
f\left(p, \bar{x}_{k+1}, \ldots, \bar{x}_{n}\right) & =\sum_{i=1}^{k} f_{i}\left(p_{i}\right)+f^{\prime}\left(\bar{x}_{k+1}, \ldots, \bar{x}_{n}\right) \\
& =\sum_{i=1}^{k} f_{i}\left(\lambda_{i} v_{i}+\left(1-\lambda_{i}\right) \bar{x}_{i}\right)+m \\
& \leq \sum_{i=1}^{k} \max \left\{f_{i}\left(v_{i}\right), f_{i}\left(\bar{x}_{i}\right)\right\}+m \\
& =\sum_{i=1}^{k} f_{i}\left(v_{i}\right)+m \leq z .
\end{aligned}
$$


Hence $\left[v,\left(\bar{x}_{1}, \ldots, \bar{x}_{k}\right)\right]_{1} \subseteq \operatorname{Proj}\left(\mathcal{L}_{\leq, f}(z)\right)_{k}$ and therefore $\operatorname{Proj}\left(\mathcal{L}_{\leq, f}(z)\right)_{k}$ is cross-shaped w.r.t. $\left(\bar{x}_{1}, \ldots, \bar{x}_{k}\right)$ for all $\bar{x} \in \overline{\bar{X}}$.

This means again that the resulting mixed-integer relaxation where the integrality constraints for $x_{k+1}, \ldots, x_{n}$ are relaxed has the Rounding Property and any $\left(v^{*}, w^{*}\right)$ gives a better bound than any $\bar{x}$, if $\bar{X} \cap\left(\mathbb{Z}^{n_{1}} \times \mathbb{R}^{n_{2}}\right)=\emptyset$.

We come back to this idea in the next section. 


\subsection{Application to convex quadratic problems}

In this section we investigate how we can use the results obtained in this chapter in the special case of convex quadratic mixed-integer problems as considered in Chapter 5. To this end we consider problems of the following form

$$
\begin{array}{lll}
(C Q M I P) & \min & q(v, w):=\left(v^{t}, w^{t}\right) Q\left(\begin{array}{c}
v \\
w
\end{array}\right)+L^{t}\left(\begin{array}{c}
v \\
w
\end{array}\right) \\
\text { s.t. } & v \in \mathbb{Z}^{n_{1}} \\
& w \in \mathbb{R}^{n_{2}}
\end{array}
$$

where $Q \in \mathbb{R}^{n_{1}+n_{2}, n_{1}+n_{2}}$ symmetric and positive definite and $L \in \mathbb{R}^{n_{1}+n_{2}}$. Chapter 5 gives us

(i) $\overline{(V, W)}=\left\{-\frac{1}{2} Q^{-1} L\right\}$.

(ii) $\mathcal{L}_{\leq, q}(z)=\sqrt{z-q(\bar{v}, \bar{w})} E\left(Q,\left(\begin{array}{c}\bar{v} \\ \bar{w}\end{array}\right)\right)$ for all $z \geq q(\bar{v}, \bar{w})$

(iii) $q(v, w)=q(\bar{v}, \bar{w})+\left((v-\bar{v})^{t},(w-\bar{w})^{t}\right) Q\left(\begin{array}{c}v-\bar{v} \\ w-\bar{w}\end{array}\right)$ for all $(v, w) \in \mathbb{R}^{n_{1}+n_{2}}$.

This means that Basic Assumption 6.4 is fulfilled: $\overline{(V, W)} \neq \emptyset$ and efficient to compute and also $\min \left\{q_{\hat{v}}(w):=q(\hat{v}, w): w \in \mathbb{R}^{n_{2}}\right\}$ is efficiently solvable for any fixed $\hat{v} \in \mathbb{Z}^{n_{1}}$ since if we partition $Q$ as

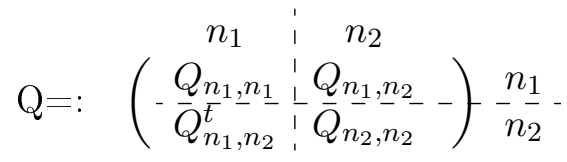

we get

$$
\begin{aligned}
q_{\hat{v}}(w)= & q(\bar{v}, \bar{w})+(\hat{v}-\bar{v})^{t} Q_{n_{1}, n_{1}}(\hat{v}-\bar{v})-2(\hat{v}-\bar{v})^{t} Q_{n_{1}, n_{2}} \bar{w} \\
& +\bar{w}^{t} Q_{n_{2}, n_{2}} \bar{w}+2\left(Q_{n_{1}, n_{2}}^{t}(\hat{v}-\bar{v})-Q_{n_{2}, n_{2}} \bar{w}\right)^{t} w+w^{t} Q_{n_{2}, n_{2}} w \\
= & c^{\prime}+2\left(Q_{n_{1}, n_{2}}^{t}(\hat{v}-\bar{v})-Q_{n_{2}, n_{2}} \bar{w}\right)^{t} w+w^{t} Q_{n_{2}, n_{2}} w
\end{aligned}
$$

where $Q_{n_{2}, n_{2}}$ is symmetric and positive definite since it is derived from $Q$ by deleting the first $n_{1}$ rows and columns and hence

$$
\hat{w}=-\frac{1}{2} Q_{n_{2}, n_{2}}^{-1}\left(2\left(Q_{n_{1}, n_{2}}^{t}(\hat{v}-\bar{v})-Q_{n_{2}, n_{2}} \bar{w}\right)\right)=-Q_{n_{2}, n_{2}}^{-1} Q_{n_{1}, n_{2}}^{t}(\hat{v}-\bar{v})+\bar{w}
$$

is the optimal solution to $\min \left\{q_{\hat{v}}(w):=q(\hat{v}, w): w \in \mathbb{R}^{n_{2}}\right\}$ as above.

To use Corollary 6.15 we first of all investigate what $g(v):=\min _{w \in \mathbb{R}^{n_{2}}} q_{v}(w)$ looks like.

Lemma 6.24. Let $q(v, w)=q(\bar{v}, \bar{w})+\left((v-\bar{v})^{t},(w-\bar{w})^{t}\right) Q\left(\begin{array}{c}v-\bar{v} \\ w-\bar{w}\end{array}\right)$ for a symmetric and positive definite $Q$, then

$$
g(v):=\min _{w \in \mathbb{R}^{n_{2}}} q_{v}(w)=q(\bar{v}, \bar{w})+(v-\bar{v})^{t} \widetilde{Q}(v-\bar{v})
$$

where $\widetilde{Q}=Q_{n_{1}, n_{1}}-Q_{n_{1}, n_{2}} Q_{n_{2}, n_{2}}^{-1} Q_{n_{1}, n_{2}}^{t}$ is again symmetric and positive definite. 
Proof. As above we get

$$
\begin{aligned}
g(v)= & q(\bar{v}, \bar{w})+(v-\bar{v})^{t} Q_{n_{1}, n_{1}}(v-\bar{v})-2(v-\bar{v})^{t} Q_{n_{1}, n_{2}} \bar{w}+\bar{w}^{t} Q_{n_{2}, n_{2}} \bar{w} \\
& +\min _{w \in \mathbb{R}^{n_{2}}}\left[2\left(Q_{n_{1}, n_{2}}^{t}(v-\bar{v})-Q_{n_{2}, n_{2}} \bar{w}\right)^{t} w+w^{t} Q_{n_{2}, n_{2}} w\right]
\end{aligned}
$$

and using $\hat{w}=\operatorname{argmin}\left\{2\left(Q_{n_{1}, n_{2}}^{t}(v-\bar{v})-Q_{n_{2}, n_{2}} \bar{w}\right)^{t} w+w^{t} Q_{n_{2}, n_{2}} w\right\}$ as above we get

$$
\begin{aligned}
& \min _{w \in \mathbb{R}^{n_{2}}}\left[2\left(Q_{n_{1}, n_{2}}^{t}(v-\bar{v})-Q_{n_{2}, n_{2}} \bar{w}\right)^{t} w+w^{t} Q_{n_{2}, n_{2}} w\right] \\
= & -(v-\bar{v})^{t} Q_{n_{1}, n_{2}} Q_{n_{2}, n_{2}}^{-1} Q_{n_{1}, n_{2}}^{t}(v-\bar{v})+2(v-\bar{v})^{t} Q_{n_{1}, n_{2}} \bar{w}-\bar{w}^{t} Q_{n_{2}, n_{2}} \bar{w} .
\end{aligned}
$$

Thus we have

$$
\begin{aligned}
g(v)= & q(\bar{v}, \bar{w})+(v-\bar{v})^{t} Q_{n_{1}, n_{1}}(v-\bar{v})-2(v-\bar{v})^{t} Q_{n_{1}, n_{2}} \bar{w}+\bar{w}^{t} Q_{n_{2}, n_{2}} \bar{w} \\
& -(v-\bar{v})^{t} Q_{n_{1}, n_{2}} Q_{n_{2}, n_{2}}^{-1} Q_{n_{1}, n_{2}}^{t}(v-\bar{v})+2(v-\bar{v})^{t} Q_{n_{1}, n_{2}} \bar{w}-\bar{w}^{t} Q_{n_{2}, n_{2}} \bar{w} \\
= & q(\bar{v}, \bar{w})+(v-\bar{v})^{t} \underbrace{\left[Q_{n_{1}, n_{1}}-Q_{n_{1}, n_{2}} Q_{n_{2}, n_{2}}^{-1} Q_{n_{1}, n_{2}}^{t}\right]}_{=: \widetilde{Q}}(v-\bar{v})
\end{aligned}
$$

and $\widetilde{Q}:=Q_{n_{1}, n_{1}}-Q_{n_{1}, n_{2}} Q_{n_{2}, n_{2}}^{-1} Q_{n_{1}, n_{2}}^{t}$ is again symmetric and positive definite:

$\left(Q_{n_{1}, n_{1}}-Q_{n_{1}, n_{2}} Q_{n_{2}, n_{2}}^{-1} Q_{n_{1}, n_{2}}^{t}\right)^{t}=Q_{n_{1}, n_{1}}-Q_{n_{1}, n_{2}} Q_{n_{2}, n_{2}}^{-1} Q_{n_{1}, n_{2}}^{t}$ since $Q_{n_{1}, n_{1}}$ and $Q_{n_{2}, n_{2}}$ are symmetric as they are derived from $Q$ by deleting either the last $n_{2}$ or the first $n_{1}$ rows and columns.

Let $v \in \mathbb{R}^{n_{1}}$ then

$$
v^{t}\left(Q_{n_{1}, n_{1}}-Q_{n_{1}, n_{2}} Q_{n_{2}, n_{2}}^{-1} Q_{n_{1}, n_{2}}^{t}\right) v=\left(v,-Q_{n_{2}, n_{2}}^{-1} Q_{n_{1}, n_{2}}^{t} v\right)^{t} Q\left(\begin{array}{c}
v \\
-Q_{n_{2}, n_{2}}^{-1} Q_{n_{1}, n_{2}}^{t} v
\end{array}\right) \geq 0
$$

as $Q$ is positive definite.

This means, consistent with Assumption 6.13, $g(v)$ exists for all $v \in \mathbb{R}^{n_{1}},(I P)_{g}$ becomes

$$
\begin{array}{lll}
(C Q I P)_{g} & \min & g(v)=q(\bar{v}, \bar{w})+(v-\bar{v})^{t} \widetilde{Q}(v-\bar{v}) \\
& \text { s.t. } \quad v \in \mathbb{Z}^{n_{1}}
\end{array}
$$

and in line with Theorem 6.14 we get $\bar{V}_{g}=\{\bar{v}\}=\operatorname{Proj}(\overline{(V, W)})_{n_{1}}$.

Remark 6.25. Lemma 6.24 means that the projection of an ellipsoid is again an ellipsoid which is neither surprising nor new. For example Karl et al. [1994] showed that

$$
\operatorname{Proj}(E(Q, 0))_{n_{1}}=E(\widetilde{Q}, 0) \text { for } \widetilde{Q}=\left(C^{t} Q^{-1} C\right)^{-1} \text { where } C=\left(\begin{array}{c}
I_{n_{1}} \\
0_{n_{2}, n_{1}}
\end{array}\right) .
$$

However we were able to derive this result just by using our theory and furthermore get a form for $\widetilde{Q}$ that fits into our framework.

The question whether $(C Q M I P)$ has the (Strong) Rounding Property hence boils down to the question whether $(C Q I P)_{g}$ has the (Strong) Rounding Property. Thus we investigate under which assumptions (on $Q) E(\widetilde{Q}, \bar{x})$ is axisparallel or quasiround. 
(i) First of all we observe that if $Q=\operatorname{Diag}(t)$ for some $t>0$ we have $\widetilde{Q}=Q_{n_{1}, n_{1}}=$ $\operatorname{Diag}\left(\left(t_{1}, \ldots, t_{n_{1}}\right)\right)$.

(ii) It is also sufficient that $Q_{n_{1}, n_{1}}=\operatorname{Diag}\left(t^{\prime}\right)$ for some $t^{\prime}>0$ and $Q_{n_{1}, n_{2}}=0_{n_{1}, n_{2}}$ which is a special case of Lemma 6.22 .

(iii) But it is also possible that $\widetilde{Q}=\operatorname{Diag}\left(t^{\prime}\right)$ for some $t^{\prime}>0$ even though $Q$ does not contain any zeros: consider for example $Q=\left(\begin{array}{lll}1 & 1 & 2 \\ 1 & 3 & 4 \\ 2 & 4 & 8\end{array}\right)$ which is symmetric and positive definite and results for $n_{1}=2$ in $\widetilde{Q}=\left(\begin{array}{ll}\frac{1}{2} & 0 \\ 0 & 1\end{array}\right)$. Note that this means also that Lemma 6.22 and 6.23 give only sufficient but not necessary conditions for the projection to be cross-shaped.

(iv) For $n_{1}=1$ we have $\widetilde{Q} \in \mathbb{R}$ and hence $g(v)=q(\bar{v}, \bar{w})+\widetilde{Q}(v-\bar{v})^{2}$ and the level sets are symmetric intervals about $\bar{v}$ which means that the problem has the Strong Rounding Property, in line with Lemma 6.17.

(v) We have already seen in Lemma 6.18 that the projection of the level set can only become rounder and this applies of course also to the case of ellipsoids: $E(\widetilde{Q}, \bar{v}) \subseteq \mathbb{R}^{n_{1}}$ is at least as round as $E(Q,(\bar{v}, \bar{w})) \subseteq \mathbb{R}^{n_{1}+n_{2}}$.

To end this chapter we apply section 6.3 to this special case of objective functions and obtain the following lemma.

Lemma 6.26. Let

$$
\begin{aligned}
& (C Q I P) \quad \min \quad q(x):=x^{t} Q x+L^{t} x \\
& \text { s.t. } x \in \mathbb{Z}^{n}
\end{aligned}
$$

be given where $Q \in \mathbb{R}^{n, n}$ is symmetric and positive definite and $L \in \mathbb{R}^{n}$. If there exists $I \subseteq\{1, \ldots, n\}, I \neq \emptyset$ such that $q_{i j}=0 \forall j \neq i$ for all $i \in I$ and $\left(-\frac{1}{2} Q^{-1} L\right)_{i \in I} \notin \mathbb{Z}^{|I|}$ we get the following hierarchy of bounds

$$
q(\bar{x})<q(\overline{\bar{x}}) \leq q\left(x^{*}\right)
$$

where $\bar{x}=-\frac{1}{2} Q^{-1} L$ is the optimal solution to the continuous relaxation, $\overline{\bar{x}}= \begin{cases}\bar{x} & \text { if } i \notin I \\ \lfloor\bar{x}\rceil & \text { if } i \in I\end{cases}$ and $x^{*}$ is an optimal solution to $(C Q I P)$.

Proof. We show that $\overline{\bar{x}}$ is an optimal solution to

$$
\begin{array}{lll}
(C Q M I P) & \min & q(x) \\
& \text { s.t. } & x_{i} \in \mathbb{Z} \forall i \in I .
\end{array}
$$

Then Lemma 6.21 proves that $q(\overline{\bar{x}}) \leq q\left(x^{*}\right)$ and since $\left(\bar{x}_{i}\right)_{i \in I} \notin \mathbb{Z}^{|I|}$ we get that $\overline{\bar{x}} \neq \bar{x}$ and hence $q(\bar{x})<q(\overline{\bar{x}})$ since $Q$ is positive definite.

To show that $\overline{\bar{x}}$ is an optimal solution to $(C Q M I P)$ we prove that $(C Q M I P)$ has the Strong Rounding Property and that $\left(\bar{x}_{i}\right)_{i \notin I}$ is an optimal solution to $\min \left\{q_{\hat{v}}(w): w \in \mathbb{R}^{n-|I|}\right\}$ for $\hat{v}=\left(\left\lfloor\bar{x}_{i}\right\rceil\right)_{i \in I}$. 
$\widetilde{Q}=Q_{I, I}-Q_{I, J} Q_{J, J}^{-1} Q_{I, J}^{t}$ where $J:=\{1, \ldots, n\} \backslash I$ and $Q_{I, J}$ is obtained from $Q$ by deleting all columns $i \notin J$ and all rows $i \notin I$ and so on.

Since $q_{i j}=0 \forall j \neq i$ for all $i \in I$, this means, $\widetilde{Q}=\operatorname{Diag}\left(\left(q_{i i}\right)_{i \in I}\right)$ and hence Lemma 5.6 reveals that $(C Q M I P)$ has the Strong Rounding Property.

As above we have that $\hat{w}=-Q_{J, J}^{-1} Q_{I, J}^{t}(\hat{v}-\bar{v})+\bar{w}$ is an optimal solution to $\min \left\{q_{\hat{v}}(w): w \in\right.$ $\left.\mathbb{R}^{n-|I|}\right\}$. Since $Q_{I, J}=0_{I, J}$, this means, $\hat{w}=\bar{w}=\left(\bar{x}_{i}\right)_{i \notin I}$.

Since

$$
\begin{aligned}
q(x) & =q(\bar{x})+(x-\bar{x})^{t} Q(x-\bar{x}) \\
& =q(\bar{x})+\left(x_{i}-\bar{x}_{i}\right)_{i \in I}^{t} Q_{I, I}\left(x_{i}-\bar{x}_{i}\right)_{i \in I}+\left(x_{j}-\bar{x}_{j}\right)_{j \in J}^{t} Q_{J, J}\left(x_{j}-\bar{x}_{j}\right)_{j \in J} \\
& =q(\bar{x})+\sum_{i \in I} q_{i i}\left(x_{i}-\bar{x}_{i}\right)^{2}+\left(x_{j}-\bar{x}_{j}\right)_{j \in J}^{t} Q_{J, J}\left(x_{j}-\bar{x}_{j}\right)_{j \in J}
\end{aligned}
$$

we observe $x_{i}^{*}=\lfloor\bar{x}\rceil_{i}$ for all $i \in I$ in this situation.

We examine the different bounds in the following example.

Example 6.27. Consider the problem

$$
\min q(x):=x^{t}\left(\begin{array}{ccccc}
7 & 0 & 0 & 0 & 0 \\
0 & 9.16 & 0 & 0 & -11.88 \\
0 & 0 & 10 & 0 & 0 \\
0 & 0 & 0 & 13 & 0 \\
0 & -11.88 & 0 & 0 & 16.09
\end{array}\right) x+(-1.4,-6.24,12,-19.5,7.82) x
$$

s.t. $\quad x \in \mathbb{Z}^{5}$.

We get $\bar{x}=-\frac{1}{2} Q^{-1} L=(0.1,0.6,-0.6,0.75,0.2)^{t}$ and $q(\bar{x})=-12.0725$. Here $I=\{1,3,4\}$ and hence $\overline{\bar{x}}=(0,0.6,-1,1,0.2)^{t}$ and $q(\overline{\bar{x}})=-9.59$.

As mentioned before $x_{i}^{*}=\left\lfloor\bar{x}_{i}\right\rceil$ for all $i \in I$. To determine $x_{j}^{*}$ for $j \in J=\{2,5\}$ we have to solve the problem

$$
\begin{aligned}
& (C Q I P)^{\prime} \min \left(w-\left(\begin{array}{c}
0.6 \\
0.2
\end{array}\right)\right)^{t}\left(\begin{array}{cc}
9.16 & -11.88 \\
-11.88 & 16.09
\end{array}\right)\left(w-\left(\begin{array}{c}
0.6 \\
0.2
\end{array}\right)\right) \\
& \text { s.t. } w \in \mathbb{Z}^{2} \text {. }
\end{aligned}
$$

The level sets of this problem are depicted in Figure 6.6.

Together we get $x^{*}=(0,-1,-1,1,-1)^{t}$ and $q\left(x^{*}\right)=-8.59$. Hence we have

$$
q(\bar{x})=-12.0725<q(\overline{\bar{x}})=-9.59<q\left(x^{*}\right)=-8.59 .
$$




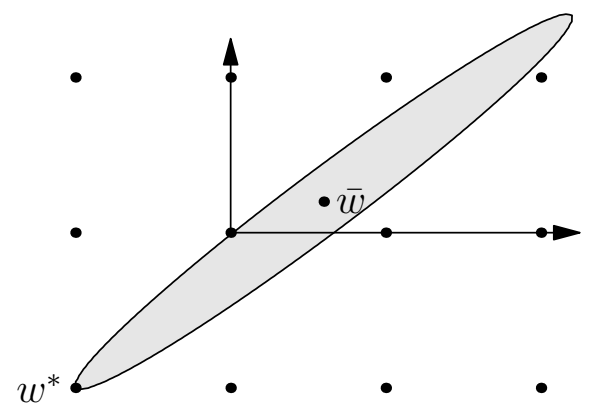

Figure 6.6.: The unique optimal solution to $(C Q I P)^{\prime}$ is $w^{*}=(-1,-1)^{t}$. 


\subsection{Summary}

In this chapter we defined a Rounding Property and a Strong Rounding Property for mixedinteger optimization problems: we say that a mixed-integer problem has the (Strong) Rounding Property if for any optimal solution $(\bar{v}, \bar{w}) \in \overline{(V, W)}$ to its continuous relaxation $(C P)$ there exists an optimal solution $\left(v^{*}, w^{*}\right) \in(V, W)^{*} \operatorname{such}$ that $v^{*} \in \operatorname{Round}(\bar{v})$ (or $v^{*}=\lfloor\bar{v}\rceil$ respectively). We showed how to adapt the algorithm we got in Chapter 1 for pure integer problems having the (Strong) Rounding Property to solve mixed-integer problems having the (Strong) Rounding Property. The difference is that in the mixed-integer case we only round the $v$ variables and hence we have to determine the corresponding $w$ variables. To this end we solve the lower dimensional continuous problem $\min \left\{f(v, w):(v, w) \in F, w \in \mathbb{R}^{n_{2}}\right\}$ for fixed $v \in \operatorname{Proj}(F)_{n_{1}} \cap \mathbb{Z}^{n_{1}}$.

In the next section we showed that a mixed-integer problem

$$
(M I P) \quad \min \left\{f(v, w):(v, w) \in F, v \in \mathbb{Z}^{n_{1}}, w \in \mathbb{R}^{n_{2}}\right\}
$$

has the (Strong) Rounding Property if and only if the lower dimensional pure integer problem $(I P)_{g} \min \left\{g(v): v \in \operatorname{Proj}(F)_{n_{1}} \cap \mathbb{Z}^{n_{1}}\right\}$ for $g(v):=\min \left\{f(v, w):(v, w) \in F, w \in \mathbb{R}^{n_{2}}\right\}$ has the (Strong) Rounding Property. The proof is based on the observation that the set of optimal solutions to $(C P)_{g}$ (the continuous relaxation of $\left.(I P)_{g}\right)$ is the projection of the set of optimal solutions to the continuous relaxation of $(M I P)$ and that the set of optimal solutions to $(I P)_{g}$ is the projection of the set of optimal solutions to $(M I P)$. This is not only true for the sets of optimal solutions but for every level set:

$$
\mathcal{L}_{\leq, g}^{\operatorname{Proj}(F)_{n_{1}}}(z)=\operatorname{Proj}\left(\mathcal{L}_{\leq, f}^{F}(z)\right)_{n_{1}} .
$$

This means that we can use the results we got in Part I to identify mixed-integer problems where the corresponding $(I P)_{g}$ has the (Strong) Rounding Property and conclude that also $(M I P)$ has the (Strong) Rounding Property.

We use this knowledge about how to identify and how to solve mixed-integer problems that have the (Strong) Rounding Property to get a lower bound on a pure integer problem: Any partition of the variables into $v$ and $w$ variables and relaxing the integrality constraint for the $w$ variables gives a relaxation of the original integer problem. If the resulting mixed-integer problem has the (Strong) Rounding Property the corresponding lower bound can easily be calculated. And if the optimal solution to the mixed-integer relaxation is not an optimal solution to the continuous relaxation this bound is strictly tighter than the bound obtained by the continuous relaxation.

In the last section we applied the results of this section to the special case of convex quadratic mixed-integer problems. In this setting we are able to give a closed formula for $g(v)$, which is again a convex quadratic function. Hence we can apply the results of Chapter 5 to $(I P)_{g}$. 



\section{Conclusion}

To end this work we summarize in this chapter the main ideas and results and give some further research ideas.

\subsection{Summary}

The general problem we considered in this work is the nonlinear integer problem

$$
(I P) \quad \min \left\{f(x): x \in F \cap \mathbb{Z}^{n}\right\}
$$

where $f: \mathbb{R}^{n} \rightarrow \mathbb{R}$ and $F \subseteq \mathbb{R}^{n}$. This general problem is NP-hard, but in many cases it is justified to assume that the continuous relaxation

$$
(C P) \min \{f(x): x \in F\}
$$

is easier to solve, compare the case of linear integer optimization. As can be seen in the linear integer problem, the assumption that the continuous relaxation is somehow easier to solve does not mean that the problem is not NP-hard anymore. In this work we assumed that there exists an efficient (not necessarily polynomial) black box algorithm to solve the continuous relaxation, meaning that we only consider problems where this basic property is fulfilled.

To use this basic assumption to solve the integer problem we defined the Rounding Property:

We say that $(I P)$ has the Rounding Property if for any optimal solution $\bar{x}$ to its continuous relaxation $(C P)$ there exists an optimal solution $x^{*} \in \operatorname{Round}(\bar{x})$, where $\operatorname{Round}(\bar{x}):=\left\{y \in \mathbb{Z}^{n}: y_{i} \in\left\{\left\lfloor x_{i}\right\rfloor,\left\lceil x_{i}\right\rceil\right\} \forall i \in\{1, \ldots, n\}\right\}$.

This means, if we know that an integer problem has the Rounding Property we can derive an optimal solution to $(I P)$ by solving $(C P)$ to get an optimal continuous minimizer $\bar{x}$ and comparing the objective values of the at most $2^{n}$ points in $\operatorname{Round}(\bar{x}) \cap F$.

Since there might be a lot of points to compare in $\operatorname{Round}(\bar{x}) \cap F$ we strengthen the Rounding Property to get the Strong Rounding Property: 
We say that $(I P)$ has the Strong Rounding Property if for any optimal solution $\bar{x}$ to its continuous relaxation $(C P)$ it holds that $\lfloor\bar{x}\rceil$ is an optimal solution to $(I P)$, where $\lfloor\bar{x}\rceil$ is the closest integer point to $\bar{x}$, made unique by any fixed tie breaking rule.

If we know that $(I P)$ has the Strong Rounding Property we can solve it by solving $(C P)$ and rounding the obtained continuous minimizer to its unique closest integer.

The question is: how do we find out whether a given nonlinear integer problem has the (Strong) Rounding Property? The approach we use here is a level set approach, i.e., we derive criteria like: "if the level sets $\mathcal{L}_{\leq, f}^{F}(z):=\{x \in F: f(x) \leq z\}$ have this special geometric shape, then the problem has the (Strong) Rounding Property". The underlying idea is the following: by the special shape of the level sets we make sure that whenever a feasible integer point $x \in \mathbb{Z}^{n} \cap F$ is in a level set $\mathcal{L}_{\leq, f}^{F}(\hat{z})$, then there is at least one rounded point $\widetilde{x} \in \operatorname{Round}(\bar{x})$ in this level set, compare Figure 7.1: due to the shape of the level sets $\widetilde{x} \in \operatorname{Round}(\bar{x})$ is among the first integer points that are reached by growing the level sets. Hence this problem has the Rounding Property.

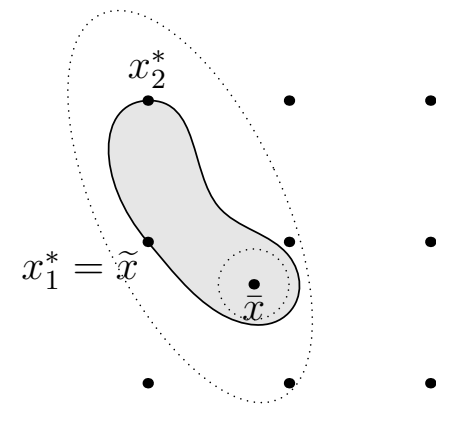

Figure 7.1.: A problem that has the Rounding Property: $\bar{x}$ is the unique continuous minimizer, the dotted lines depict level sets $\mathcal{L}_{\leq, f}(z)$ for different levels $z$ and the light gray area is $\mathcal{L}_{\leq, f}\left(f\left(x^{*}\right)\right)$.

The same approach can be used to guarantee the Strong Rounding Property: here we have to make sure that whenever a feasible integer point is in a level set, also $\lfloor\bar{x}\rceil$ is in this level set.

Since these properties might not be easy to test, we identified features of level sets ensuring this property, that are hopefully easier to check. First of all, we observe that we do not have to restrict all level sets, but only the first ones until we reach the first integer point. Of course we do not know the first integer point, since this is the optimal solution to $(I P)$ we are looking for. Therefore we relax this condition and assume that the level sets have the required feature until the first feasible point in $\operatorname{Round}(\bar{x})$ (or $\lfloor\bar{x}\rceil$ respectively) is reached.

The first feature we introduced is being quasi-round with respect to the continuous minimizer $\bar{x}$. The definition means geometrically that a set is not too different from a ball centered at $\bar{x}$ : given a fixed value $\alpha$, a level set $\mathcal{L}_{\leq, f}(z) \subseteq \mathbb{R}^{n}$ is $\alpha$-quasi-round w.r.t. $\bar{x}$, if there exists a radius $r \in \mathbb{R}_{0}^{+}$such that the ball centered at $\bar{x}$ with radius $r$ is completely contained in $\mathcal{L}_{\leq, f}(z)$ and the ball centered in $\bar{x}$ with radius $r+\alpha$ completely contains $\mathcal{L}_{\leq, f}(z)$, compare Figure 7.2. 


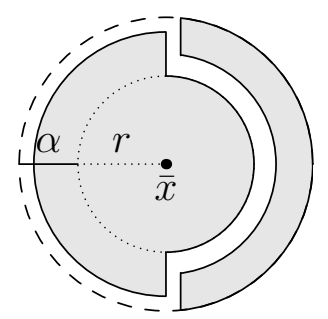

Figure 7.2.: An example for an $\alpha$-quasi-round set with respect to $\bar{x}$.

This way we make sure that whenever an integer point $x$ is in $\mathcal{L}_{\leq, f}(z)$, it is also in the ball with radius $r+\alpha$ and if we choose $\alpha$ small enough, this means, $r \geq \min _{\widetilde{x} \in \operatorname{Round}(\bar{x}) \cap F}\|\bar{x}-\widetilde{x}\|_{2}$ (or $r \geq\|\bar{x}-\lfloor\bar{x}\rceil\|_{2}$ respectively) and we are done. This is not only possible for Euclidean balls but for any $p$-norm balls. The main task in the corresponding chapter was hence to determine the right size of $\alpha$ to ensure either the Rounding Property or the Strong Rounding Property. Furthermore, we were able to adapt $\alpha$ to guarantee also other proximity results of the form: for each optimal solution $\bar{x}$ to the continuous relaxation there exists an optimal solution $x^{*}$ to (IP) such that $\left\|\bar{x}-x^{*}\right\|_{\infty} \leq c$ for a fixed $c \in \mathbb{N}$.

The second way we identified of ensuring the Rounding Property is to require the level sets to be cross-shaped with respect to the continuous minimizer $\bar{x}$ : whenever a point $y$ is in $\mathcal{L}_{\leq, f}(z)$ also the axisparallel box given by $y$ and $\bar{x}$ is in $\mathcal{L}_{\leq, f}(z)$, compare Figure 7.3.

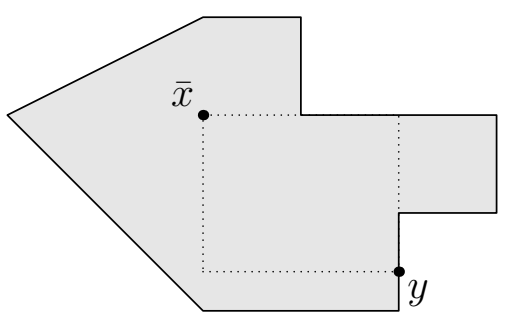

Figure 7.3.: An example for a cross-shaped set w.r.t. $\bar{x}$.

If $y$ is an integer point there is always at least one of the rounded points in this box and hence we are done. To even get the Strong Rounding Property we have to assume furthermore that the level sets are symmetric with respect to the hyperplanes parallel to the coordinate axes through $\bar{x}$. Since pictures like the one in Figure 7.3 might suggest a connection to biconvexity, we investigated the connection to the definitions of convexity, biconvexity and ortho-convexity. Furthermore, we introduced the concept of quasi-cross-shapedness, which is an adaption of quasi-roundness: we do not assume that the set can be enclosed between two balls but between two scaled versions of a cross-shaped set.

Geometrically, we can interpret quasi-roundness and cross-shapedness in the following way: using the right $\alpha$ in the definition of $\alpha$-quasi-roundness we use the size of the integer lattice to determine a shape that leads to the Rounding Property. On the other hand, for the definition of cross-shapedness we use the orientation of the lattice. This explains also why quasi-roundness is not preserved under scaling and cross-shapedness not under rotation. 
In the second part of this work we showed an application to unconstrained convex quadratic integer problems and an extension to mixed-integer problems. The special case of convex quadratic integer problems is an interesting field of application for our theory since the continuous relaxation can be solved extremely easily and the continuous minimizer is unique. Furthermore, we know that the level sets are ellipsoids whose shape is determined only by the Hessian of the quadratic objective function. Nevertheless, the unconstrained convex quadratic integer problem is still NP-hard. We investigated when ellipsoids are cross-shaped, quasi-round or quasi-cross-shaped with respect to their center. But, in addition, we also explored how to use our ideas if the original problem does not have the Rounding Property. In those cases we use a convex quadratic underestimator to the original objective function that has the Strong Rounding Property. Using this underestimator we get a lower bound (that is better then the continuous relaxation) to use in a branch-and-bound tree. We developed several bounds and embedded them in the branch-and-bound framework by Buchheim et al. [2012a]. Some first computational results show that at least one of our bounds outperforms the bound originally used by Buchheim et al. [2012a]. Our other bounds are stricter but their computation time is too long.

If not all but only some of the variables have to be integer, we say that a mixed-integer problem has the Rounding Property if for any optimal solution to its continuous relaxation there exists an optimal solution to the mixed-integer problem such that the integer variables are among the rounded coordinates of the corresponding variables of the continuous minimizer. The main result of the corresponding chapter is that a mixed-integer problem has the (Strong) Rounding Property if and only if its projection onto the integer variables has the (Strong) Rounding Property. We used this result to identify problems where a mixed-integer bound can be found by rounding some of the variables of the continuous relaxation and therefore outperforms the bound obtained by the continuous relaxation.

\subsection{Further research directions}

An obvious field of open questions is the search for other shapes, as quasi-round and crossshaped, that guarantee the Rounding Property. In the light of our experiences we expect that this requires some link between the shape in question and the integer lattice, compare also the discussion about quasi-roundness and cross-shapedness in the previous section.

Another really interesting question is whether it would be of any help if the level sets are not quasi-round or cross-shaped with respect to the continuous minimizer, but with respect to some other point. (This is, at least for the cross-shaped case, not possible for the set of continuous minimizers $\bar{X}$, but as mentioned in Chapter 4 we could relax the assumptions anyway.) A possible application for this setting could be a perturbed $p$-norm where, in contrast to Section 2.3.1, we do not force the continuous minimizer to stay the same. If the perturbation is not too large, we might get that the level sets are quasi-round with respect to the continuous minimizer of the unperturbed problem. This would imply that we can get an optimal solution to the perturbed integer problem by rounding the unperturbed continuous minimizer and hence without determining the perturbed continuous minimizer. It seems worth to think further about this question.

Next we mention two questions that are closely connected to the results and concepts presented in this work. 
In Section 3.5 we introduced the concept of quasi-cross-shapedness. As mentioned the challenge is to determine the appropriate $\epsilon$ to guarantee the Rounding Property. We were able to calculate this value for the special cases of quasi-roundness and quasi-axisparallelity. The question is whether there are other special cases for which this is also possible. One could think for example of something like quasi-rectangular shaped.

In Chapter 5 we used underestimators that have the Strong Rounding Property to calculate lower bounds to use in a branch-and-bound setting. In the enumeration tree we can stop branching as soon as we obtain a problem that has the Strong Rounding Property, since we can easily solve it to optimality. Remember that in each depth of the tree the Hessian of the objective function (that defines the shape of the corresponding level sets) only depends on the depth in the tree and is obtained from the Hessian of the original problem by deleting some rows and columns. The question is hence whether it is possible to fix the variables in an order that leads as soon as possible to separable objective functions or functions with quasi-round level sets and hence to the Strong Rounding Property.

Another more fundamental research direction could be to investigate the following problem: assume that we know that a given problem has the Rounding Property. Now we are given the chance to demand a specific continuous minimizer of the black-box algorithm we use to solve the continuous relaxation. Which one should we choose? The aim is of course to make $|F \cap \operatorname{Round}(\bar{x})|$ as small as possible in order to have few integer points to compare. A possible solution could be to ask for a continuous minimizer that has a lot of integer components, but maybe it is even better to take one that is close to or even on the boundary of $F$.

Last but not least we have to mention that we need to keep looking for more applications to test our approach. The difficulty is most of the time that we assume a lot of knowledge about the shapes of the level sets. Nevertheless, we have seen in Chapter 5 that the fact that we know what the level sets look like does not necessarily mean that the problem is easy to solve in general. A promising approach might also be to approximate the level sets in a certain neighborhood of the continuous minimizer, for example by considering the level sets of a Taylor approximation. The important task is then of course to guarantee that not only the approximation but also the original function has the Rounding Property.

Looking for applications might also lead to the interesting question whether there are properties of problems that guarantee the (Strong) Rounding Property and that can be tested by a computer. 



\section{Frequently used notation}

General notation

$\begin{array}{cl}\mathbb{Z} & \text { integer numbers } \\ \mathbb{Z}^{n} & \text { integer lattice of dimension } n \\ \mathbb{R} & \text { real numbers } \\ \mathbb{R}_{0}^{+} & \text {non-negative real numbers } \\ \mathbb{R}^{n} & \text { Euclidean space of dimension } n \\ \mathbb{R}^{n, n} & \text { set of real } n \times n \text {-matrices }\end{array}$

Rounding Property

$(I P) \quad$ (pure) integer program

$(M I P) \quad$ mixed-integer program

$(C P) \quad$ continuous prgramm, used here for the continuous relaxation

$(f, F) \quad \min \{f(x): x \in F\}$ possibly with further integrality constraints if mentioned

$X^{*} \quad$ set of optimal solutions to $(I P)$ or $(M I P)$

$x^{*} \quad$ an optimal solution to $(I P)$

$\bar{X} \quad$ set of optimal solutions to $(C P)$

$\bar{x} \quad$ an optimal solution to $(C P)$

RP Rounding Property

SRP Strong Rounding Property

For $x \in \mathbb{R}$

$\lfloor x\rfloor \quad=\max \{y \in \mathbb{Z}: y \leq x\}$

$\lceil x\rceil \quad=\min \{y \in \mathbb{Z}: y \geq x\}$

$\lfloor x\rceil \quad=\operatorname{argmin}\{|x-y|: y \in \mathbb{Z}\}$ made unique by any fixed tie-breaking rule

$\operatorname{Round}(x)=\{\lfloor x\rfloor,\lceil x\rceil\}$ 
For $x \in \mathbb{R}^{n}$

$$
\begin{aligned}
\lfloor x\rfloor & =\left(\left\lfloor x_{1}\right\rfloor, \ldots,\left\lfloor x_{n}\right\rfloor\right) \\
\lceil x\rceil & =\left(\left\lceil x_{1}\right\rceil, \ldots,\left\lceil x_{n}\right\rceil\right) \\
\lfloor x\rceil & =\left(\left\lfloor x_{1}\right\rceil, \ldots,\left\lfloor x_{n}\right\rceil\right)\left(\in \operatorname{argmin} d_{p}\left(x, \mathbb{Z}^{n}\right)\right) \\
\lfloor x\rceil^{F} & \in \operatorname{argmin} d_{p}\left(x, \mathbb{Z}^{n} \cap F\right) \\
\underset{\operatorname{Round}(x)}{\widetilde{x}} & =\left\{y \in \mathbb{Z}^{n}: y_{i} \in \operatorname{Round}\left(x_{i}\right)\right\} \\
\widetilde{x}^{F} & \in \operatorname{argmin} d_{p}\left(x, \mathbb{Z}^{n} \backslash \operatorname{Round}(x)\right) \\
\hat{x} & \in \operatorname{argmin} d_{p}\left(x,\left(\mathbb{Z}^{n} \cap F\right) \backslash \operatorname{Round}(x)\right) \\
\hat{x}^{F} & \in \operatorname{argmin} d_{p}\left(x, \mathbb{Z}^{n} \backslash\{\lfloor x\rceil\}\right) \\
&
\end{aligned}
$$

Sets

$$
\begin{aligned}
\mathcal{L}_{\leq, f}^{D}(z)= & \{x \in D: f(x) \leq z\}, \text { for } f: D \rightarrow \mathbb{R}, z \in \mathbb{R} \\
B_{p}(x, r)= & \left\{y \in \mathbb{R}^{n}:\|x-y\|_{p} \leq r\right\}, \text { for } x \in \mathbb{R}^{n}, r \in \mathbb{R}_{0}^{+}, p \in[1, \infty] \\
{[x, y]_{d} } & \left\{z \in \mathbb{R}^{n}: d(x-z)+d(z-y)=d(x-y)\right\}, \\
& \text { for } x, y \in \mathbb{R}^{n}, d: \mathbb{R}^{n} \rightarrow \mathbb{R} \text { a norm } \\
E(Q, x) \quad= & \left\{y \in \mathbb{R}^{n}:(y-x)^{\top} Q(y-x) \leq 1\right\}, \\
& \quad \text { for } Q \in \mathbb{R}^{n, n} \text { positive semidefinite, } x \in \mathbb{R}^{n}
\end{aligned}
$$

$\operatorname{Proj}(M)_{m}=\left\{x \in \mathbb{R}^{m}: \exists y \in \mathbb{R}^{n-m}:(x, y) \in M\right\}$, for $M \subseteq \mathbb{R}^{n}$ where $n>m$

Distances

$$
\begin{aligned}
d_{p}(x, M) & =\inf \left\{\|x-y\|_{p}: y \in M\right\}, \text { for } x \in \mathbb{R}^{n}, M \subseteq \mathbb{R}^{n}, p \in[1, \infty] \\
\|x\|_{Q} & =\sqrt{x^{\top} Q x}, \text { for } Q \in \mathbb{R}^{n, n} \text { positive semidefinite, } x \in \mathbb{R}^{n} \\
\|x\|_{t} & =\sqrt{x^{\top} \operatorname{Diag}(t) x}, \text { for } t \geq 0, x \in \mathbb{R}^{n}
\end{aligned}
$$




\section{Summary of contributions}

In this section we outline which of the results presented in this work have been derived in cooperation with others and which results are part of submitted papers.

Section 1.2 and Section 1.3: Most of the given definitions as well as Lemma 1.18 are already submitted in Hübner and Schöbel [2013].

Chapter 2: The definition of quasi-roundness, the connection between the quasi-roundness of the level sets and the Rounding Property as well as the calculation of $\alpha_{p}(\bar{x})$ and $\alpha_{p}$ are already submitted in Hübner and Schöbel [2013]. The idea to adapt $\alpha_{p}$ to even get the Strong Rounding Property is joint work with Anita Schöbel and Christoph Buchheim and the corresponding theorem as well as the calculation of $\beta_{p}(\bar{x})$ can be found in Buchheim et al. [2013]. The examples in Section 2.3.1 are also submitted in Hübner and Schöbel [2013].

Chapter 3: The definition of cross-shapedness and the connection between cross-shaped level sets and the (Strong) Rounding Property are also given in Hübner and Schöbel [2013]. Also the results about separable convex functions and absolute norms can be found there. Section 3.4.3 benefited from the correspondence with Arie Tamir.

Chapter 5: This chapter is joint work with Anita Schöbel and Christoph Buchheim, compare Buchheim et al. [2013]. The computational results have been done by Christoph. 



\section{Bibliography}

Adams, W. P., Forrester, R. J., and Glover, F. W. (2004). Comparisons and enhancement strategies for linearizing mixed 0-1 quadratic programs. Discrete Optimization, 1(2):99-120.

Adams, W. P. and Sherali, H. D. (1986). A tight linearization and an algorithm for zero-one quadratic programming problems. Management Science, 32(10):1274-1290.

Aissi, H., Bazgan, C., and Vanderpooten, D. (2009). Min-max and min-max regret versions of combinatorial optimization problems: A survey. European Journal of Operational Research, $197(2): 427-438$.

Baes, M., del Pia, A., Nesterov, Y., Onn, S., and Weismantel, R. (2013). Minimizing lipschitzcontinuous strongly convex functions over integer points in polytopes. Mathematical Programming, 134(1):305-322.

Bank, B., Krick, T., Mandel, R., and Solernó, P. (1991). A geometrical bound for integer programming with polynomial constraints. In Fundamentals of Computation Theory, pages 121-125. Springer.

Barahona, F., Jünger, M., and Reinelt, G. (1989). Experiments in quadratic 0-1 programming. Mathematical Programming, 44(1-3):127-137.

Bauer, F. L., Stoer, J., and Witzgall, C. (1961). Absolute and monotonic norms. Numerische Mathematik, 3(1):257-264.

Ben-Tal, A., den Hertog, D., and Vial, J. (2012). Deriving robust counterparts of nonlinear uncertain inequalities.

Ben-Tal, A., el Ghaoui, L., and Nemirovski, A. (2009). Robust optimization. Princeton University Press.

Ben-Tal, A. and Nemirovski, A. (1998). Robust convex optimization. Mathematics of Operations Research, 23(4):769-805.

Ben-Tal, A. and Nemirovski, A. (2002). Robust optimization - methodology and applications. Mathematical Programming, 92(3):453-480. 
Benders, J. F. (1962). Partitioning procedures for solving mixed-variables programming problems. Numerische mathematik, 4(1):238-252.

Billionnet, A. and Elloumi, S. (2007). Using a mixed integer quadratic programming solver for the unconstrained quadratic 0-1 problem. Mathematical Programming, 109(1):55-68.

Billionnet, A., Elloumi, S., and Lambert, A. (2012). Extending the qcr method to general mixed-integer programs. Mathematical programming, 131(1-2):381-401.

Billionnet, A., Elloumi, S., and Lambert, A. (2013). An efficient compact quadratic convex reformulation for general integer quadratic programs. Computational Optimization and Applications, 54(1):141-162.

Billionnet, A., Elloumi, S., and Plateau, M.-C. (2009). Improving the performance of standard solvers for quadratic $0-1$ programs by a tight convex reformulation: The qcr method. Discrete Applied Mathematics, 157(6):1185-1197.

Boltyanski, V., Martini, H., and Soltan, P. (1996). Star-shaped sets in normed spaces. Discrete \&6 Computational Geometry, 15(1):63-71.

Bomze, I. M. and Locatelli, M. (2004). Undominated d.c. decomposition of quadratic functions and applications to branch-and-bound approaches. Computational Optimization and Applications, 28(2):227-245.

Bonami, P., Biegler, L. T., Conn, A. R., Cornuéjols, G., Grossmann, I. E., Laird, C. D., Lee, J., Lodi, A., Margot, F., Sawaya, N., and Wächter, A. (2008). An algorithmic framework for convex mixed integer nonlinear programs. Discrete Optimization, 5(2):186-204.

Bonami, P., Kilinç, M., and Linderoth, J. (2012). Algorithms and software for convex mixed integer nonlinear programs. In Mixed Integer Nonlinear Programming, pages 1-39. Springer.

Borchers, B. and Mitchell, J. E. (1994). An improved branch and bound algorithm for mixed integer nonlinear programs. Computers $\& 3$ Operations Research, 21(4):359-367.

Buchheim, C., Caprara, A., and Lodi, A. (2012a). An effective branch-and-bound algorithm for convex quadratic integer programming. Mathematical Programming, 135(1-2):369-395.

Buchheim, C., de Santis, M., Palagi, L., and Piacentini, M. (2012b). An exact algorithm for quadratic integer minimization using nonconvex relaxations. Department of Computer and System Sciences Antonio Ruberti Technical Reports, 4(5).

Buchheim, C., Hübner, R., and Schöbel, A. (2013). Ellipsoid bounds for convex quadratic integer programming. Technical report, Institute for Numerical and Applied Mathematics, University of Göttingen.

Buchheim, C. and Traversi, E. (2013). Separable non-convex underestimators for binary quadratic programming. In Experimental Algorithms, pages 236-247. Springer.

Buchheim, C. and Wiegele, A. (2012). Semidefinite relaxations for non-convex quadratic mixed-integer programming. Mathematical Programming, pages 1-18.

Butt, S. E. and Cavalier, T. M. (1996). An efficient algorithm for facility location in the presence of forbidden regions. European Journal of Operations Research, 90(1):56-70. 
Büsing, C., Goetzmann, K., and Matuschke, J. (2011). Compromise solutions in multicriteria combinatorial optimization. Technical report, Technical University Berlin.

Caprara, A. and Fischetti, M. (1997). Branch-and-cut algorithms. Annotated bibliographies in combinatorial optimization, pages 45-63.

Carter, M. W. (1984). The indefinite zero-one quadratic problem. Discrete Applied Mathematics, $7(1): 23-44$.

Dakin, R. J. (1965). A tree-search algorithm for mixed integer programming problems. The Computer Journal, 8(3):250-255.

Drezner, Z., Klamroth, K., Schöbel, A., and Wesolowsky, G. O. (2002). The weber problem, pages 1-36. in: Facility location: Applications and theory. Springer-Verlag.

Duran, M. A. and Grossmann, I. E. (1986). An outer-approximation algorithm for a class of mixed-integer nonlinear programs. Mathematical programming, 36(3):307-339.

Dvoretzky, A. (1963). Some near-sphericity results. In Proc. Symposia in Pure Math, volume 7, pages 203-210.

Eyster, J. W. and White, J. A. (1973). Some properties of the squared euclidean distance location problem. AIIE Transactions, 5(3):275-280.

Fincke, U. and Pohst, M. (1985). Improved methods for calculating vectors of short length in a lattice, including a complexity analysis. Mathematics of Computation, 44(170):463-471.

Fletcher, R. and Leyffer, S. (1994). Solving mixed integer nonlinear programs by outer approximation. Mathematical programming, 66(1-3):327-349.

Fortet, R. (1960). L'algebre de boole et ses applications en recherche opérationnelle. Trabajos de Estadistica y de Investigación Operativa, 11(2):111-118.

Garey, M. R. and Johnson, D. S. (1979). Computers and Intractability: A Guide to the Theory of NP-completeness, volume 174. Freeman New York.

Geoffrion, A. M. (1970). Elements of large-scale mathematical programming. Management Science, 16(11):652-675.

Geoffrion, A. M. (1972). Generalized benders decomposition. Journal of optimization theory and applications, 10(4):237-260.

Glover, F. (1975). Improved linear integer programming formulations of nonlinear integer problems. Management Science, 22(4):455-460.

Goerigk, M. (2013). Algorithms and concepts for robust optimization.

Gomory, R. E. (1958). Outline of an algorithm for integer solutions to linear programs. Bulletin of the American Mathematical Society, 64(5):275-278.

Gorski, J., Pfeuffer, F., and Klamroth, K. (2007). Biconvex sets and optimization with biconvex functions - a survey and extensions. Mathematical Methods of Operations Research, 66(3):373-407. 
Granot, F. and Skorin-Kapov, J. (1990). Some proximity and sensitivity results in quadratic integer programming. Mathematical Programming, 47(1-3):259-268.

Graver, J. E. (1975). On the foundations of linear and integer linear programming i. Mathematical Programming, 9(1):207-226.

Grossmann, I. E. (2002). Review of nonlinear mixed-integer and disjunctive programming techniques. Optimization and Engineering, 3(3):227-252.

Grossmann, I. E. and Kravanja, Z. (1995). Mixed-integer nonlinear programming techniques for process systems engineering. Computers $\&$ chemical engineering, 19:189-204.

Grzybowski, J., Nickel, S., Pallaschke, D., and Urbanski, R. (2011). Ordered median functions and symmetries. Optimization, 60(7):801-811.

Gupta, O. K. and Ravindran, A. (1985). Branch and bound experiments in convex nonlinear integer programming. Management Science, 31(12):1533-1546.

Hamacher, H. W. and Nickel, S. (1995). Restricted planar location problems and applications. Naval Research Logistics, 42(6):967-992.

Hamacher, H. W. and Schöbel, A. (1997). A note on center problems with forbidden polyhedra. Operations research letters, 20(4):165-169.

Hammer, P. L. and Rubin, A. A. (1970). Some remarks on quadratic programming with 0-1 variables. RAIRO-Operations Research-Recherche Opérationnelle, 4(V3):67-79.

Helmberg, C. (2000). Fixing variables in semidefinite relaxations. SIAM Journal on Matrix Analysis and Applications, 21(3):952-969.

Helmberg, C. and Rendl, F. (1998). Solving quadratic (0, 1)-problems by semidefinite programs and cutting planes. Mathematical Programming, 82(3):291-315.

Hemmecke, R. (2003). Test sets for integer programs with z-convex objective. arXiv preprint math/0309154.

Hemmecke, R., Köppe, M., Lee, J., and Weismantel, R. (2010). Nonlinear Integer Programming, chapter 15, pages 561-618. in: 50 years of integer programming. Springer.

Hochbaum, D. S. and Shanthikumar, J. G. (1990). Convex separable optimization is not much harder than linear optimization. Journal of the Association for Computing Machinery, $37(4): 843-862$.

Hübner, R. and Schöbel, A. (2013). When is rounding allowed in integer nonlinear optimization? submitted.

Jeroslow, R. G. (1973). There cannot be any algorithm for integer programming with quadratic constraints. Operations Research, 21(1):221-224.

Johnson, C. R. and Nylen, P. (1991). Monotonicity properties of norms. Linear Algebra and its Applications, 148:43-58. 
Johnson, E. L., Nemhauser, G. L., and Savelsbergh, M. W. P. (2000). Progress in linear programming-based algorithms for integer programming: An exposition. INFORMS Journal on Computing, 12(1):2-23.

Jünger, M., Liebling, T., Naddef, D., Nemhauser, G., Pulleyblank, W., Reinelt, G., Rinaldi, G., and Wolsey, L. (2010). 50 Years of Integer Programming 1958-2008. Springer, Berlin.

Karl, W. C., Verghese, G. C., and Willsky, A. S. (1994). Reconstructing ellipsoids from projections. CVGIP: Graphical Models and Image Processing, 56(2):124-139.

Katz, I. N. and Cooper, L. (1981). Facility location in the presence of forbidden regions, i: Formulation and the case of euclidean distance with one forbidden circle. European Journal of Operations Research, 6(2):166-173.

Kelley, J. E. (1960). The cutting-plane method for solving convex programs. Journal of the Society for Industrial \&3 Applied Mathematics, 8(4):703-712.

Khachiyan, L. G. (1983). Convexity and complexity in polynomial programming. In Proceedings of the International Congress of Mathematicians, pages 1569-1577.

Körner, M.-C. (2011). Minisum Hyperspheres, volume 51 of Springer Optimization and Its Applications. Springer.

Kouvelis, P. and Yu, G. (1997). Robust Discrete Optimization and Its Applications, volume 14. Springer.

Land, A. H. and Doig, A. G. (1960). An automatic method of solving discrete programming problems. Econometrica: Journal of the Econometric Society, pages 497-520.

Lee, J., Onn, S., and Weismantel, R. (2008). On test sets for nonlinear integer maximization. Operations Research Letters, 36(4):439-443.

Lenstra, H. W. (1983). Integer programming with a fixed number of variables. Mathematics of operations research, pages 538-548.

Leyffer, S. (2001). Integrating sqp and branch-and-bound for mixed integer nonlinear programming. Computational Optimization and Applications, 18(3):295-309.

Lodi, A. (2010). Mixed integer programming computation. In 50 Years of Integer Programming 1958-2008, pages 619-645. Springer.

McBride, R. D. and Yormark, J. S. (1980). An implicit enumeration algorithm for quadratic integer programming. Management Science, 26(3):282-296.

Nemhauser, G. L. and Wolsey, L. A. (1988). Integer and combinatorial optimization, volume 18. Wiley New York.

Pardalos, P. M. and Rodgers, G. P. (1990). Computational aspects of a branch and bound algorithm for quadratic zero-one programming. Computing, 45(2):131-144.

Pardalos, P. M. and Vavasis, S. A. (1991). Quadratic programming with one negative eigenvalue is np-hard. Journal of Global Optimization, 1:15-22. 
Plastria, F. (2002). Continuous covering location problems, pages 37-79. in: Facility location: Applications and theory. Springer-Verlag.

Puerto, J. and Nickel, S. (2005). Location Theory - A Unified Approach. Springer.

Quesada, I. and Grossmann, I. E. (1992). An lp/nlp based branch and bound algorithm for convex minlp optimization problems. Computers 86 chemical engineering, 16(10):937-947.

Rawlins, G. J. E. and Wood, D. (1988). Ortho-convexity and its generalizations, pages 137-152. in: Computational Morphology. Elsevier Science Publishers.

Rendl, F. (1999). Semidefinite programming and combinatorial optimization. Applied Numerical Mathematics, 29(3):255-281.

Rendl, F., Rinaldi, G., and Wiegele, A. (2010). Solving max-cut to optimality by intersecting semidefinite and polyhedral relaxations. Mathematical Programming, 121(2):307-335.

Stubbs, R. A. and Mehrotra, S. (1999). A branch-and-cut method for 0-1 mixed convex programming. Mathematical Programming, 86(3):515-532.

Thompson, A. C. (1996). Minkowski Geometry. Cambridge University Press, Cambridge.

van Emde-Boas, P. (1981). Another NP-complete partition problem and the complexity of computing short vectors in a lattice. University of Amsterdam, Department of Mathematics.

Ward, J. E. and Wendell, R. E. (1980). A new norm for measuring distance which yields linear location problems. Operations Research, 28(3-Part-II):836-844.

Ward, J. E. and Wendell, R. E. (1985). Using block norms for location modeling. Operations Research, 33(5):1074-1090.

Weismantel, R. (1998). Test sets of integer programs. Mathematical Methods of Operations Research, 47(1):1-37.

Westerlund, T. and Pettersson, F. (1995). An extended cutting plane method for solving convex minlp problems. Computers $\mathscr{G}$ Chemical Engineering, 19:131-136.

Westerlund, T. and Pörn, R. (2002). Solving pseudo-convex mixed integer optimization problems by cutting plane techniques. Optimization and Engineering, 3(3):253-280.

Yu, G. (1996). On the max-min 0-1 knapsack problem with robust optimization applications. Operations Research, 44(2):407-415.

Yu, G. and Yang, J. (1998). On the robust shortest path problem. Computers \& Operations Research, 25(6):457-468. 


\section{Index}

$\epsilon$-similarity, 85

Rounding Property, 92

Strong Rounding Property, 93

Weakened Rounding Property, 94

axisparallel, 104

biconvex, 66

coordinate axially symmetric, $61,64,65$

cross-shaped, 53, 54, 59

ellipsoids, 103, 104

Rounding Property, 58

Strong Rounding Property, 62

lower bounds for (CQIP), 125

average-case, 123

axisparallel, 114

quasi-axisparallel, 118

quasi-round, 114

worst-case, 121

norm, 75

absolute, 75

block norm, 77

cornered p-norm, 77

elliptic, 78

weighted one-infinity norm, 77

weighted version, 77

ordered median, 71

Rounding Property, 71

ortho-convex, 66 quasi-axisparallel, 107

ellipsoids, 108, 109

quasi-cross-shaped, 85

Rounding Property, 85, 91

quasi-round, 23

ellipsoids, 105, 106

proximity result, 47,48

Rounding Property, 26, 27, 29, 30

Strong Rounding Property, 36, 37

quasiconvex

Strong Rounding Property, 69

Rounding Property, 10

$n=1,19$

algorithm, 12

mixed-integer, 134, 141

$n=1,142$

algorithm, 134

reformulation, 15

separable quasiconvex, 71

Rounding Property, 71

Strong Rounding Property, 13

$n=1,19$

algorithm, 13

mixed-integer, 136, 141

$n=1,142$

reformulation, 15

Weakened Rounding Property, 93, 94 